\title{
Palladium-Catalyzed Cascade Arylation of Vinylogous Esters Enabled by Tris(1-adamantyl)phosphine
}

\author{
Yi-Chi Yang, Yi-Ching Lin and Yen-Ku Wu* \\ Department of Applied Chemistry, Nation Chiao Tung University, 1001 University Road, Hsinchu 30010, Taiwan \\ E-mail: yenkuwu@nctu.edu.tw
}

\section{Supporting Information}

Table of Contents

General Information.......................................................................

Synthesis of Previously Known Compounds................................................S-1

Synthesis and Characterization Data of $2 \mathrm{a}-\mathrm{c}, 3 \mathrm{3}-3 \mathrm{z}, 4 \mathrm{a}-\mathrm{h}$, and 5-8..........................26

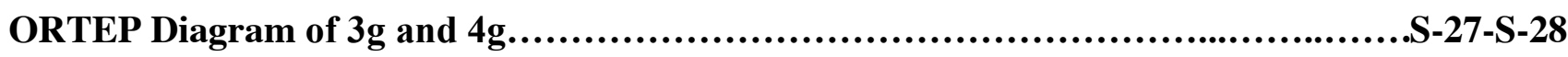

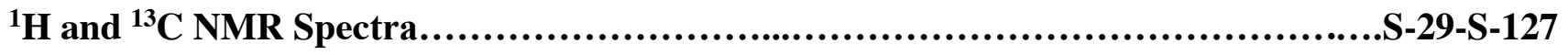




\section{General Information}

All air-sensitive reactions were carried out using flame-dried glassware under $\mathrm{N}_{2}$ atmosphere with standard Schlenk line technique. Toluene, 1,4-dioxane, tetrahydrofuran, dichloromethane were purified by passage over activated alumina using a commercial solvent purification system. All other solvents (ACS grade) and commercially obtained reagents were used as received. Aluminum heating blocks were applied to all thermal reactions. Hexamethyldisilazane was freshly distilled over potassium hydroxide. A solution of Lithium hexamethyldisilazide (LiHMDS) was prepared prior to use by deprotonating bis(trimethylsilyl)amine in THF with $n$-butyllithium $(2.5 \mathrm{M}$ in hexanes). Reactions were monitored by thin layer chromatography (TLC) on silica gel $60 \AA$ F254 plates, visualized by UV (254 $\mathrm{nm})$ and $\mathrm{KMnO}_{4}$ staining solution. Flash chromatography was performed on silica gel (230-400 mesh) with indicated eluents. Melting points were uncorrected. NMR spectra were measured at $400 \mathrm{MHz}$ for ${ }^{1} \mathrm{H}$ spectra, $100 \mathrm{MHz}$ for ${ }^{13} \mathrm{C}$ spectra and $375 \mathrm{MHz}$ for ${ }^{19} \mathrm{~F}$ spectra and calibrated from residual solvent signals (chloroform at $7.26 \mathrm{ppm}$ for ${ }^{1} \mathrm{H}$ spectra; chloroform at 77.16 ppm for ${ }^{13} \mathrm{C}$ spectra; trifluoroacetic acid as internal standard at $-76.55 \mathrm{ppm}$ for ${ }^{19} \mathrm{~F}$ spectra). Chemical shifts were denoted in ppm $(\delta)$, and the following abbreviations were used to explain the multiplicities: $\mathrm{s}=$ singlet, $\mathrm{br}=$ broad, brs $=$ broad singlet, $\mathrm{brd}=$ broad doublet, $\mathrm{d}=$ doublet, $\mathrm{t}=$ triplet, $\mathrm{q}=$ quartet, $\mathrm{p}$ $=$ pentate, $\mathrm{dd}=$ doublet doublet, $\mathrm{td}=$ triple doublet, $\mathrm{dt}=$ double triplet, $\mathrm{m}=$ multiplet. Coupling constants $(J)$ are reported in Hertz (Hz). Infrared (IR) spectra were measured on KBr salt plates. Highresolution mass spectroscopy (HRMS) was performed on a TOF instrument with EI or FD in positive ionization mode.

\section{Synthesis of Previously Known Compounds}

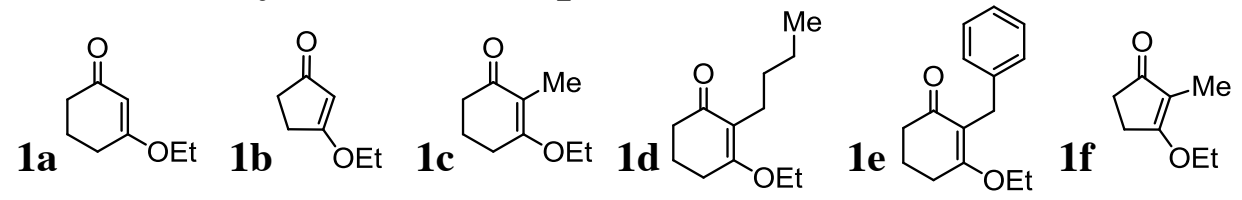

Compounds above were prepared by following previously described procedures:

For synthesis of compounds 1a, 1b, 1c, 1d and 1f, see: Johnson, T.; Pultar, F.; Menke, F.; Lautens, M. Org. Lett. 2016, 18, 6488-6491.

For synthesis of compound 1e, see: Carruthers, W.; Cumming, S. A. J. Chem. Soc., Perkin Trans. 1 1983, 10, 2383-2386. 


\section{Synthesis and Characterization Data of 2a-c, 3a-3z, 4a-h, and 5-8}

Evaluation of Conditions for the Cascade Diarylation (Table 1).

6-(Benzo[d][1,3]dioxol-5-yl)-3-ethoxycyclohex-2-en-1-one (2a)

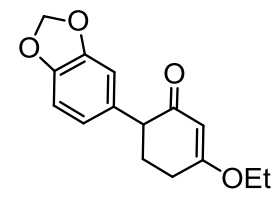

A round-bottom flask containing a vinylogous ester $1 \mathbf{a}(1.0 \mathrm{mmol}), 5$-bromo-1,3-benzodioxole (1.2 equiv, $242 \mathrm{mg}), \mathrm{Pd}(\mathrm{dba})_{2}(3 \mathrm{~mol} \%, 18 \mathrm{mg})$ and $\mathrm{PAd}_{3}(3.4 \mathrm{~mol} \%, 14 \mathrm{mg})$ was vacuumed for 2 minutes. The whole system was backfilled with $\mathrm{N}_{2}$, and then $10 \mathrm{~mL}$ of toluene was added to the flask. To the stirring mixture was added a solution of LiHMDS ( $1 \mathrm{M}, 2.0$ equiv, $3.2 \mathrm{~mL})$ over 5 minutes at room temperature. The resulting mixture was then stirred at $70{ }^{\circ} \mathrm{C}$ (temperature of the aluminum heating block) for 30 minutes. After completion of the reaction as indicated by TLC analysis, a saturated aqueous solution of $\mathrm{NH}_{4} \mathrm{Cl}$ was added to quench the reaction at room temperature. The reaction mixture was filtered through celite. The filtrate was diluted with ethyl acetate and extracted with water. The organic layers were dried over anhydrous $\mathrm{MgSO}_{4}$, filtered and concentrated under reduced pressure. The crude residue thus obtained was purified by flash column chromatography (hexanes/EtOAc $=8 / 1$ to 3/1) to afford 2a $(188 \mathrm{mg}, 72 \%$ in entry 4$)$ as yellow solid (m.p. $\left.72-73{ }^{\circ} \mathrm{C}\right)$. $\mathrm{R}_{f}: 0.5$ (hexanes/ EtOAc = 1:1). IR (cast): 2939, 1654, 1606, $1504 \mathrm{~cm}^{-1} ;{ }^{1} \mathbf{H} \mathbf{N M R}\left(400 \mathrm{MHz}, \mathrm{CDCl}_{3}\right)$ : $\delta 6.76(\mathrm{~d}, J=7.9 \mathrm{~Hz}, 1 \mathrm{H}), 6.65(\mathrm{~d}, J=1.8 \mathrm{~Hz}, 1 \mathrm{H}), 6.63(\mathrm{dd}, J=7.9,1.8 \mathrm{~Hz}, 1 \mathrm{H}), 5.93(\mathrm{~d}, J=1.7 \mathrm{~Hz}$, 2H), $5.49(\mathrm{~s}, 1 \mathrm{H}), 3.97-3.90(\mathrm{~m}, 2 \mathrm{H}), 3.44(\mathrm{dd}, J=10.2,5.0 \mathrm{~Hz}, 1 \mathrm{H}), 2.59-2.41(\mathrm{~m}, 2 \mathrm{H}), 2.28-2.10(\mathrm{~m}$, 2H), 1.38 (t, $J=7.0 \mathrm{~Hz}, 3 \mathrm{H}) ;{ }^{13} \mathbf{C}$ NMR (100 MHz, $\left.\mathrm{CDCl}_{3}\right): \delta 199.44,177.47,147.83,146.55,133.81$, 121.66, 108.89, 108.45, 103.18, 101.07, 64.58, 51.89, 29.75, 28.51, 14.31; HRMS (EI, [M] ${ }^{+}$) for $\mathrm{C}_{15} \mathrm{H}_{16} \mathrm{O}_{4}$ calcd. 260.1043, found: 260.1044 .

\section{Evaluation of Conditions for the Cascade Diarylation (Table 1).}

6,6-Bis(benzo[d][1,3]dioxol-5-yl)-3-ethoxycyclohex-2-en-1-one (3a)

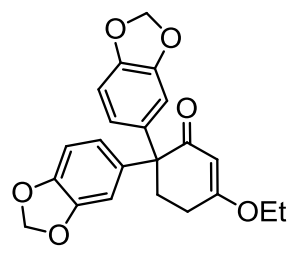


A round-bottom flask containing a vinylogous ester $1 \mathrm{a}(1.0 \mathrm{mmol}), 5$-bromo-1,3-benzodioxole (3.0 equiv, $605 \mathrm{mg}), \mathrm{Pd}(\mathrm{OAc})_{2}(10 \mathrm{~mol} \%, 22 \mathrm{mg})$ and $\mathrm{PAd}_{3}(12 \mathrm{~mol} \%, 52 \mathrm{mg})$ was vacuumed for 2 minutes. The whole system was backfilled with $\mathrm{N}_{2}$, and then $10 \mathrm{~mL}$ of toluene was added to the flask. To the stirring mixture was added a solution of LiHMDS (1 M, 3.0 equiv, $4.8 \mathrm{~mL})$ over 5 minutes at room temperature. The resulting mixture was then stirred at $70{ }^{\circ} \mathrm{C}$ (temperature of the aluminum heating block) for 30 minutes. After completion of the reaction as indicated by TLC analysis, a saturated aqueous solution of $\mathrm{NH}_{4} \mathrm{Cl}$ was added to quench the reaction at room temperature. The reaction mixture was filtered through celite. The filtrate was diluted with ethyl acetate and extracted with water. The organic layers were dried over anhydrous $\mathrm{MgSO}_{4}$, filtered and concentrated under reduced pressure. The crude residue thus obtained was purified by flash column chromatography (hexanes/EtOAc $=8 / 1$ to 3/1) to afford 3a $(288 \mathrm{mg}, 76 \%$ in entry 8$)$ as yellow solid (m.p. 175-176 $\left.{ }^{\circ} \mathrm{C}\right) . \mathrm{R}_{f}: 0.5$ (hexanes/ EtOAc $\left.=1 / 1\right) . \mathbf{I R}$ (cast): 3072, 2899, 1650, 1608, $1486 \mathrm{~cm}^{-1} ;{ }^{1} \mathbf{H}$ NMR $(400$ $\left.\mathrm{MHz}, \mathrm{CDCl}_{3}\right): \delta 6.73(\mathrm{~d}, J=8.1 \mathrm{~Hz}, 2 \mathrm{H}), 6.63(\mathrm{~d}, J=1.8 \mathrm{~Hz}, 2 \mathrm{H}), 6.60(\mathrm{dd}, J=8.1,1.8 \mathrm{~Hz}, 2 \mathrm{H}), 5.93$ (s, 4H), $5.50(\mathrm{~s}, 1 \mathrm{H}), 3.88(\mathrm{q}, J=7.0 \mathrm{~Hz}, 2 \mathrm{H}), 2.60$ (t, $J=5.9 \mathrm{~Hz}, 2 \mathrm{H}), 2.36(\mathrm{t}, J=5.9 \mathrm{~Hz}, 2 \mathrm{H}), 1.33$ (t, $J=7.0 \mathrm{~Hz}, 3 \mathrm{H}) ;{ }^{13} \mathbf{C}$ NMR $\left(100 \mathrm{MHz}, \mathrm{CDCl}_{3}\right): \delta 199.99,176.40,147.69,146.49,136.37,121.65$, 109.54, 107.81, 103.26, 101.17, 64.55, 58.13, 34.50, 27.30, 14.27; HRMS (EI, [M] $]^{+}$) for $\mathrm{C}_{22} \mathrm{H}_{20} \mathrm{O}_{6}$ calcd. 380.1254 , found: 380.1258 .

\section{Two-Step Sequential Arylations (Scheme 2).}

\section{4-Ethoxy-5,6-dihydro-[1,1'-biphenyl]-2(1H)-one (2b)}

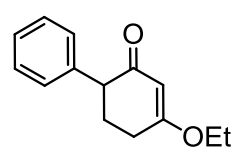

A round-bottom flask containing a vinylogous ester $1 \mathbf{a}(0.5 \mathrm{mmol})$, bromobenzene (1.2 equiv, 94 $\mathrm{mg}), \mathrm{Pd}(\mathrm{dba})_{2}(3 \mathrm{~mol} \%, 9 \mathrm{mg})$ and $\mathrm{TTBP}^{\mathrm{H}} \cdot \mathrm{HBF}_{4}$ (tri-tert-butylphosphonium tetrafluoroborate; $3.4 \mathrm{~mol}$ $\%, 5 \mathrm{mg}$ ) was vacuumed for 2 minutes. The whole system was backfilled with $\mathrm{N}_{2}$, and then $5 \mathrm{~mL}$ of toluene was added to the flask. To the stirring mixture was added a solution of LiHMDS (1 M, 2.0 equiv, $1.6 \mathrm{~mL}$ ) over 3 minutes at room temperature. The resulting mixture was then stirred at $70{ }^{\circ} \mathrm{C}$ (temperature of the aluminum heating block) for 30 minutes. After completion of the reaction as indicated by TLC, a saturated aqueous solution of $\mathrm{NH}_{4} \mathrm{Cl}$ was added to quench the reaction at room 
temperature. The reaction mixture was filtered through celite. The filtrate was diluted with ethyl acetate and extracted with water. The organic layers were dried over anhydrous $\mathrm{MgSO}_{4}$, filtered and concentrated under reduced pressure. The crude residue thus obtained was purified by flash column chromatography (hexanes/EtOAc $=10 / 1$ to $3 / 1)$ to afford $\mathbf{2 b}(81 \mathrm{mg}, 75 \%)$ as yellow solid (m.p. 44$\left.45^{\circ} \mathrm{C}\right) . \mathrm{R}_{f}: 0.3$ (hexanes/ EtOAc $\left.=3 / 1\right) . \mathbf{I R}\left(\right.$ cast): 3028, 2939, 1656, 1606, $1496 \mathrm{~cm}^{-1} ;{ }^{1} \mathbf{H}$ NMR $(400$ $\left.\mathrm{MHz}, \mathrm{CDCl}_{3}\right): \delta 7.32(\mathrm{t}, J=7.3 \mathrm{~Hz}, 2 \mathrm{H}), 7.27-7.22(\mathrm{~m}, 1 \mathrm{H}), 7.18(\mathrm{~d}, J=7.1 \mathrm{~Hz}, 2 \mathrm{H}), 5.51(\mathrm{~s}, 1 \mathrm{H})$, $3.94(\mathrm{q}, J=7.0 \mathrm{~Hz}, 2 \mathrm{H}), 3.53(\mathrm{dd}, J=9.6,5.3 \mathrm{~Hz}, 1 \mathrm{H}), 2.58-2.41(\mathrm{~m}, 2 \mathrm{H}), 2.30-2.16(\mathrm{~m}, 2 \mathrm{H}), 1.39(\mathrm{t}$, $J=7.0 \mathrm{~Hz}, 3 \mathrm{H}) ;{ }^{13} \mathbf{C}$ NMR $\left(100 \mathrm{MHz}, \mathrm{CDCl}_{3}\right): \delta 199.35,177.40,140.05,128.63,128.45,126.93$, 103.29, 64.52, 52.09, 29.53, 28.34, 14.30; HRMS (EI, [M] $]^{+}$) for $\mathrm{C}_{14} \mathrm{H}_{16} \mathrm{O}_{2}$ calcd. 216.1145, found: 216.1142 .

\section{4'-Ethoxy-3,4-dimethoxy-5',6'-dihydro-2' $H$-[1,1':1',1''-terphenyl]-2'-one (3b)}

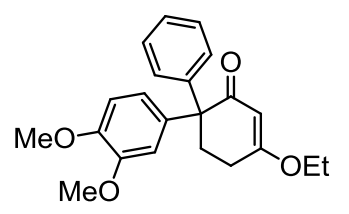

A round-bottom flask containing $\mathbf{2 b}(0.5 \mathrm{mmol}), 4$-bromoveratrole (1.5 equiv, $163 \mathrm{mg}), \mathrm{Pd}(\mathrm{OAc})_{2}$ $(10 \mathrm{~mol} \%, 11 \mathrm{mg})$ and $\operatorname{PAd}_{3}(12 \mathrm{~mol} \%, 26 \mathrm{mg})$ was vacuumed for 2 minutes. The whole system was backfilled with $\mathrm{N}_{2}$, and then $5 \mathrm{~mL}$ of toluene was added to the flask. To the stirring mixture was added a solution of LiHMDS ( $1 \mathrm{M}, 1.5$ equiv, $1.2 \mathrm{~mL}$ ) over 3 minutes at room temperature. The resulting mixture was then stirred at $70{ }^{\circ} \mathrm{C}$ (temperature of the aluminum heating block) for 30 minutes. After completion of the reaction, a saturated aqueous solution of $\mathrm{NH}_{4} \mathrm{Cl}$ was added to quench the reaction at room temperature. The reaction mixture was filtered through celite. The filtrate was diluted with ethyl acetate and extracted with water. The organic layers were dried over anhydrous $\mathrm{MgSO}_{4}$, filtered and concentrated under reduced pressure. The crude residue thus obtained was purified by flash column chromatography (hexanes/EtOAc $=8 / 1$ to $3 / 1)$ to afford $\mathbf{3 b}(148 \mathrm{mg}, 84 \%)$ as yellow oil. $\mathrm{R}_{f}: 0.4$ (hexanes/ EtOAc = 1/1). IR (film): 3058, 2953, 2835, 1654, 1609, $1517 \mathrm{~cm}^{-1}$; ${ }^{\mathbf{1}} \mathbf{H}$ NMR (400 MHz, $\left.\mathrm{CDCl}_{3}\right): \delta 7.31-7.11(\mathrm{~m}, 3 \mathrm{H}), 7.12(\mathrm{~d}, J=7.3 \mathrm{~Hz}, 2 \mathrm{H}), 6.81(\mathrm{~d}, J=8.1 \mathrm{~Hz}, 1 \mathrm{H}), 6.71(\mathrm{~d}, J=8.8 \mathrm{~Hz}$, 2H), $5.54(\mathrm{~s}, 1 \mathrm{H}), 3.90(\mathrm{~d}, J=7.0 \mathrm{~Hz}, 2 \mathrm{H}), 3.86(\mathrm{~s}, 3 \mathrm{H}), 3.76(\mathrm{~s}, 3 \mathrm{H}), 2.69$ (t, $J=5.8 \mathrm{~Hz}, 2 \mathrm{H}), 2.46-$ $2.32(\mathrm{~m}, 2 \mathrm{H}), 1.32(\mathrm{t}, J=7.0 \mathrm{~Hz}, 3 \mathrm{H}) ;{ }^{13} \mathbf{C}$ NMR (100 MHz, $\left.\mathrm{CDCl}_{3}\right): \delta 200.33,176.38,148.69,148.04$, 
$143.07,134.27,128.58,128.19,126.89,120.78,112.43,110.63,103.45,64.50,58.38,55.96,55.91$, 34.35, 27.34, 14.26; HRMS (EI, [M] ${ }^{+}$) for $\mathrm{C}_{22} \mathrm{H}_{24} \mathrm{O}_{4}$ calcd. 352.1669, found: 352.1673 .

\section{General Procedure A for the Cascade Diarylation (Scheme 3).}

A round-bottom flask containing a vinylogous ester $(0.5 \mathrm{mmol})$, an aryl bromide (3.0 equiv), $\mathrm{Pd}(\mathrm{OAc})_{2}(10 \mathrm{~mol} \%, 11 \mathrm{mg})$ and $\mathrm{PAd}_{3}(12 \mathrm{~mol} \%, 26 \mathrm{mg})$ was vacuumed for 2 minutes. The whole system was backfilled with $\mathrm{N}_{2}$, and then $5 \mathrm{~mL}$ of toluene was added to the flask. To the stirring mixture was added a solution of LiHMDS (1 M, 3.0 equiv, $2.4 \mathrm{~mL})$ over 3 minutes at room temperature. The resulting mixture was then stirred at $70^{\circ} \mathrm{C}$ (temperature of the aluminum heating block) for 30 minutes. After completion of the reaction as indicated by TLC, a saturated aqueous solution of $\mathrm{NH}_{4} \mathrm{Cl}$ was added to quench the reaction at room temperature. The reaction mixture was filtered through celite. The filtrate was diluted with ethyl acetate and extracted with water. The organic layers were dried over anhydrous $\mathrm{MgSO}_{4}$, filtered and concentrated under reduced pressure. The crude residue thus obtained was purified by flash column chromatography.

\section{4'-Ethoxy-5',6'-dihydro-2' $H$-[1,1':1',1'-terphenyl]-2'-one (3c)}

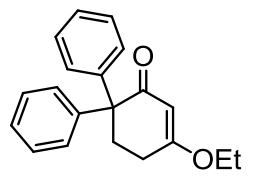

The reaction was conducted with $0.5 \mathrm{mmol}$ of $\mathbf{1 a}$ following the general procedure A. The crude product was purified by flash column chromatography (hexanes/EtOAc $=10 / 1$ to $3 / 1)$ to afford $\mathbf{3 c}(111 \mathrm{mg}$, $76 \%$ ) as yellow solid (m.p. $134-135{ }^{\circ} \mathrm{C}$ ). $\mathrm{R}_{f}: 0.48$ (hexanes/ EtOAc $=3 / 1$ ). IR (cast): 3058, 2953, 1654, 1609, $1421 \mathrm{~cm}^{-1} ;{ }^{1} \mathbf{H}$ NMR (400 MHz, $\left.\mathrm{CDCl}_{3}\right): \delta$ 7.43-7.33 (m, 7H), 7.27-7.23 (m, 3H), 5.66 (s, 1H), 3.99 (q, $J=7.0 \mathrm{~Hz}, 2 \mathrm{H}), 2.83(\mathrm{t}, J=6.0 \mathrm{~Hz}, 2 \mathrm{H}), 2.47(\mathrm{t}, J=6.0 \mathrm{~Hz}, 2 \mathrm{H}), 1.43$ (t, $J=7.0 \mathrm{~Hz}$, 3H); ${ }^{13} \mathrm{C}$ NMR (100 MHz, $\left.\mathrm{CDCl}_{3}\right): \delta 200.05,176.37,142.44,128.69,128.23,126.94,103.52,64.51$, 58.73, 34.12, 27.28, 14.27; HRMS (EI, [M] $]^{+}$) for $\mathrm{C}_{20} \mathrm{H}_{20} \mathrm{O}_{2}$ calcd. 292.1458, found: 292.1454. 


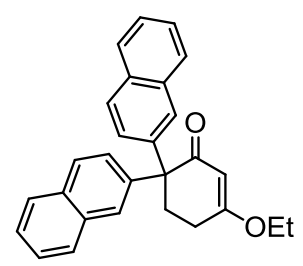

The reaction was conducted with $0.5 \mathrm{mmol}$ of $\mathbf{1 a}$ following the general procedure $\mathrm{A}$. The crude product was purified by flash column chromatography (hexanes/EtOAc $=10 / 1$ to 3/1) to afford $\mathbf{3 d}$ (194 mg, $99 \%$ ) as orange solid (m.p. $75-76^{\circ} \mathrm{C}$ ). $\mathrm{R}_{f}: 0.38$ (hexanes/ EtOAc = 3/1). IR (cast): 3055, 2980, 1651, 1607, $1379 \mathrm{~cm}^{-1} ;{ }^{1} \mathbf{H}$ NMR (400 MHz, $\left.\mathrm{CDCl}_{3}\right): \delta$ 7.88-7.83 (m, 3H), $7.81(\mathrm{~s}, 1 \mathrm{H}), 7.81-7.77(\mathrm{~m}, 2 \mathrm{H})$, $7.68(\mathrm{~s}, 2 \mathrm{H}), 7.49(\mathrm{dd}, J=6.5,2.7 \mathrm{~Hz}, 4 \mathrm{H}), 7.40(\mathrm{dd}, J=8.7,2.0 \mathrm{~Hz}, 2 \mathrm{H}), 5.70(\mathrm{~s}, 1 \mathrm{H}), 3.92(\mathrm{q}, J=$ $7.0 \mathrm{~Hz}, 2 \mathrm{H}), 2.96(\mathrm{t}, J=5.9 \mathrm{~Hz}, 2 \mathrm{H}), 2.45(\mathrm{t}, J=5.9 \mathrm{~Hz}, 2 \mathrm{H}), 1.35(\mathrm{t}, J=7.0 \mathrm{~Hz}, 3 \mathrm{H}) ;{ }^{13} \mathbf{C} \mathbf{~ N M R}(100$ $\left.\mathrm{MHz}, \mathrm{CDCl}_{3}\right): \delta 199.95,176.60,139.81,133.15,132.42,128.28,127.75,127.51,127.23,126.08$, 126.05, 103.49, 64.53, 58.85, 33.79, 27.33, 14.19; HRMS (EI, [M] $]^{+}$) for $\mathrm{C}_{28} \mathrm{H}_{24} \mathrm{O}_{2}$ calcd. 392.1771, found: 392.1775 .

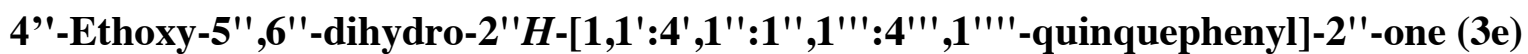

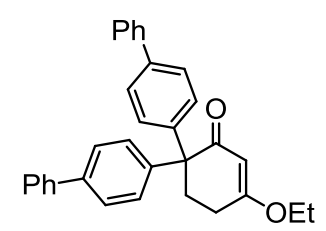

The reaction was conducted with $0.5 \mathrm{mmol}$ of $\mathbf{1 a}$ following the general procedure A. The crude product was purified by flash column chromatography (hexanes/EtOAc $=10 / 1$ to $3 / 1)$ to afford $\mathbf{3 e}(218 \mathrm{mg}$, $98 \%$ ) as yellow oil. $\mathrm{R}_{f}$ : 0.4 (hexanes/ EtOAc = 3/1). IR (film): 2933, 1650, 1602, $1485 \mathrm{~cm}^{-1}$; ${ }^{1} \mathbf{H}$ NMR (400 MHz, $\left.\mathrm{CDCl}_{3}\right): \delta$ 7.61-7.55 (m, 8H), 7.45-7.41 (m, 4H), 7.35-7.32 (m, 2H), 7.27 (d, J=8.3 Hz, 4H), $5.61(\mathrm{~s}, 1 \mathrm{H}), 3.91(\mathrm{q}, J=7.0 \mathrm{~Hz}, 2 \mathrm{H}), 2.80(\mathrm{t}, J=5.9 \mathrm{~Hz}, 2 \mathrm{H}), 2.44$ (t, $J=5.8 \mathrm{~Hz}, 2 \mathrm{H}), 1.35$ (t, $J$ $=7.0 \mathrm{~Hz}, 3 \mathrm{H}) ;{ }^{13} \mathbf{C}$ NMR $\left(100 \mathrm{MHz}, \mathrm{CDCl}_{3}\right): \delta 200.06,176.57,141.44,140.80,139.78,129.09,128.87$, $127.38,127.20,126.98,103.53,64.60,58.35,34.14,27.34,14.28$; HRMS (EI, [M] $]^{+}$) for $\mathrm{C}_{32} \mathrm{H}_{28} \mathrm{O}_{2}$ calcd. 444.2084, found: 444.2080. 
4'-Ethoxy-5",6"'-dihydro-2" H-[1,1':3',1"':1",1'"':3'"',1'"'-quinquephenyl]-2' '-one (3f)

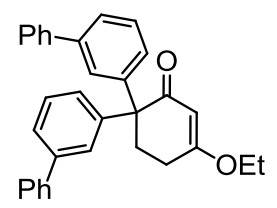

The reaction was conducted with $0.5 \mathrm{mmol}$ of $\mathbf{1 a}$ following the general procedure A. The crude product was purified by flash column chromatography (hexanes/EtOAc $=10 / 1$ to 3/1) to afford $\mathbf{3 f}(224 \mathrm{mg}, 99$ \%) as white solid (m.p. $69-70{ }^{\circ} \mathrm{C}$ ). $\mathrm{R}_{f}: 0.4$ (hexanes/ EtOAc =3/1). IR (cast): 3028, 2953, 1652, 1607, $1478 \mathrm{~cm}^{-1}$; ${ }^{1} \mathbf{H}$ NMR (400 MHz, $\left.\mathrm{CDCl}_{3}\right)$ : $1 \mathrm{H} \mathrm{NMR} \mathrm{(400} \mathrm{MHz,} \mathrm{Chloroform-d)} \delta 7.53$ (d, J=8.1 Hz, 4H), $7.50(\mathrm{~d}, J=7.7 \mathrm{~Hz}, 2 \mathrm{H}), 7.44-7.36(\mathrm{~m}, 8 \mathrm{H}), 7.34-7.29(\mathrm{~m}, 2 \mathrm{H}), 7.16$ (d, $J=7.8 \mathrm{~Hz}, 2 \mathrm{H}), 5.60$ (s, $1 \mathrm{H}), 3.90(\mathrm{q}, J=7.0 \mathrm{~Hz}, 2 \mathrm{H}), 2.83(\mathrm{t}, J=5.8 \mathrm{~Hz}, 2 \mathrm{H}), 2.44(\mathrm{t}, J=5.7 \mathrm{~Hz}, 2 \mathrm{H}), 1.33(\mathrm{t}, J=6.9 \mathrm{~Hz}$, 3H); ${ }^{13}$ C NMR (100 MHz, $\left.\mathrm{CDCl}_{3}\right): \delta 199.91,176.47,142.84,141.39,141.16,128.82,128.67,127.88$, $127.49,127.42,127.36,125.97,103.60,64.57,58.94,34.37,27.39,14.27$; HRMS (EI, [M] $]^{+}$) for $\mathrm{C}_{32} \mathrm{H}_{28} \mathrm{O}_{2}$ calcd. 444.2084, found: 444.2081.

4'-Ethoxy-4,4''-dimethyl-5',6'-dihydro-2' $H$-[1,1':1',1'-terphenyl]-2'-one (3g)

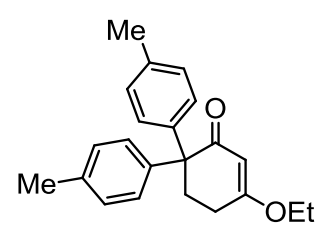

The reaction was conducted with $0.5 \mathrm{mmol}$ of $1 \mathrm{a}$ following the general procedure A. The crude product was purified by flash column chromatography (hexanes/EtOAc $=10 / 1$ to $3 / 1)$ to afford $\mathbf{3 g}(119 \mathrm{mg}$, $74 \%$ ) as yellow solid (m.p. 126-127 ${ }^{\circ} \mathrm{C}$ ). $\mathrm{R}_{f}: 0.4$ (hexanes/ EtOAc = 3/1). IR (cast): 3052, 2981, 1656, 1609, $1454 \mathrm{~cm}^{-1} ;{ }^{1} \mathbf{H}$ NMR $\left(400 \mathrm{MHz}, \mathrm{CDCl}_{3}\right): \delta 7.12(\mathrm{~d}, J=8.1 \mathrm{~Hz}, 4 \mathrm{H}), 7.04(\mathrm{~d}, J=7.4 \mathrm{~Hz}, 4 \mathrm{H})$, $5.55(\mathrm{~s}, 1 \mathrm{H}), 3.88(\mathrm{q}, J=7.0 \mathrm{~Hz}, 2 \mathrm{H}), 2.70(\mathrm{t}, J=6.0 \mathrm{~Hz}, 2 \mathrm{H}), 2.39-2.35(\mathrm{~m}, 2 \mathrm{H}), 2.33(\mathrm{~s}, 6 \mathrm{H}), 1.33$ (t, $J=7.0 \mathrm{~Hz}, 3 \mathrm{H}) ;{ }^{13} \mathbf{C}$ NMR $\left(100 \mathrm{MHz}, \mathrm{CDCl}_{3}\right): \delta 200.41,176.24,139.50,136.41,128.91,128.47$, 103.42, 64.39, 58.07, 34.08, 27.27, 21.08, 14.24; HRMS (EI, [M] $]^{+}$) for $\mathrm{C}_{22} \mathrm{H}_{24} \mathrm{O}_{2}$ calcd. 320.1771, found: 320.1767 . 
4,4'-Bis(dimethylamino)-4'-ethoxy-5',6'-dihydro-2' $H$-[1,1':1',1'-terphenyl]-2'-one (3h)

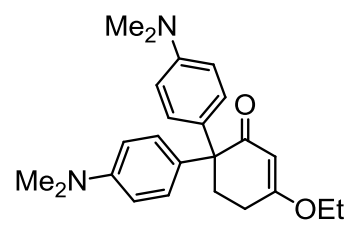

The reaction was conducted with $0.5 \mathrm{mmol}$ of $\mathbf{1 a}$ following the general procedure A. The crude product was purified by flash column chromatography (hexanes/EtOAc $=10 / 1$ to 3/1) to afford $\mathbf{3 h}(96 \mathrm{mg}, 50$ \%) as dark green solid (m.p. 157-158 ${ }^{\circ} \mathrm{C}$ ). $\mathrm{R}_{f}: 0.45$ (hexanes/ EtOAc = 2/1). IR (cast): 2952, 2927, 1654, 1611, 1519, $1184 \mathrm{~cm}^{-1} ;{ }^{1} \mathbf{H}$ NMR (400 MHz, $\left.\mathrm{CDCl}_{3}\right): \delta 7.00(\mathrm{~d}, J=7.5 \mathrm{~Hz}, 4 \mathrm{H}), 6.66(\mathrm{~d}, J=8.0$ $\mathrm{Hz}, 4 \mathrm{H}), 5.51(\mathrm{~s}, 1 \mathrm{H}), 3.85(\mathrm{q}, J=7.0 \mathrm{~Hz}, 2 \mathrm{H}), 2.92(\mathrm{~s}, 12 \mathrm{H}), 2.63(\mathrm{t}, J=5.6 \mathrm{~Hz}, 2 \mathrm{H}), 2.37(\mathrm{t}, J=5.9$ $\mathrm{Hz}, 2 \mathrm{H}), 1.30(\mathrm{t}, J=7.0 \mathrm{~Hz}, 3 \mathrm{H}) ;{ }^{13} \mathbf{C}$ NMR $\left(100 \mathrm{MHz}, \mathrm{CDCl}_{3}\right): \delta 201.39,176.04,149.26,130.70$, 129.23, 112.29, 103.44, 64.26, 57.16, 40.69, 34.30, 27.44, 14.29; HRMS (EI, [M] $]^{+}$) for $\mathrm{C}_{24} \mathrm{H}_{30} \mathrm{~N}_{2} \mathrm{O}_{2}$ calcd. 378.2302, found: 378.2308 .

4'-Ethoxy-3,3",4,4' '-tetramethoxy-5',6'-dihydro-2' $H$-[1,1':1',1'-terphenyl]-2' -one (3i)

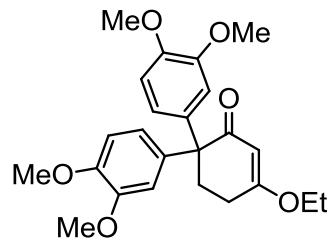

The reaction was conducted with $0.5 \mathrm{mmol}$ of $1 \mathrm{a}$ following the general procedure A. The crude product was purified by flash column chromatography (hexanes/EtOAc $=8 / 1$ to 3/1) to afford $\mathbf{3 i}(144 \mathrm{mg}, 70$ \%) as brown oil. $\mathrm{R}_{f}: 0.3$ (hexanes/ EtOAc $=1 / 1$ ). IR (film): 3066, 2953, 1653, 1609, $1515 \mathrm{~cm}^{-1} ;{ }^{1} \mathbf{H}$ NMR (400 MHz, $\left.\mathrm{CDCl}_{3}\right): \delta 6.79(\mathrm{~d}, \mathrm{~J}=9.0 \mathrm{~Hz}, 2 \mathrm{H}), 6.66(\mathrm{dd}, \mathrm{J}=5.8,2.1 \mathrm{~Hz}, 7 \mathrm{H}), 5.52(\mathrm{~s}, 1 \mathrm{H}), 3.90$ $3.86(\mathrm{~m}, 2 \mathrm{H}), 3.85(\mathrm{~s}, 6 \mathrm{H}), 3.76(\mathrm{~s}, 6 \mathrm{H}), 2.65(\mathrm{t}, J=6.0 \mathrm{~Hz}, 2 \mathrm{H}), 2.40(\mathrm{t}, J=6.0 \mathrm{~Hz}, 2 \mathrm{H}), 1.32(\mathrm{t}, J=$ $7.0 \mathrm{~Hz}, 3 \mathrm{H}) ;{ }^{13} \mathrm{C}$ NMR $\left(100 \mathrm{MHz}, \mathrm{CDCl}_{3}\right): \delta 200.55,176.39,148.60,147.95,134.91,120.72,112.28$, 110.59, 103.36, 64.47, 58.03, 55.95, 55.88, 34.56, 27.36, 14.23; HRMS (EI, [M] ${ }^{+}$) for $\mathrm{C}_{24} \mathrm{H}_{28} \mathrm{O}_{6}$ calcd. 412.1880, found: 412.1888 . 
4'-Ethoxy-4,4''-dimethoxy-5',6'-dihydro-2' $H$-[1,1':1',1'-terphenyl]-2'-one (3j)

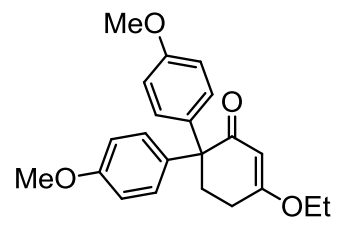

The reaction was conducted with $0.5 \mathrm{mmol}$ of $\mathbf{1 a}$ following the general procedure A. The crude product was purified by flash column chromatography (hexanes/EtOAc $=10 / 1$ to 3/1) to afford $\mathbf{3 j}$ ( $95 \mathrm{mg}, 54$ \%) as brown oil. $\mathrm{R}_{f}: 0.3$ (hexanes/ EtOAc = 3/1). IR (film): 2952, 2908, 1653, 1609, $1510 \mathrm{~cm}^{-1} ;{ }^{1} \mathbf{H}$ NMR (400 MHz, $\left.\mathrm{CDCl}_{3}\right): \delta 7.05(\mathrm{~d}, J=8.9 \mathrm{~Hz}, 4 \mathrm{H}), 6.83(\mathrm{~d}, J=8.9 \mathrm{~Hz}, 4 \mathrm{H}), 5.52(\mathrm{~s}, 1 \mathrm{H}), 3.87$ (q, $J$ $=7.1 \mathrm{~Hz}, 2 \mathrm{H}), 3.78(\mathrm{~s}, 6 \mathrm{H}), 2.65(\mathrm{t}, J=6.0 \mathrm{~Hz}, 2 \mathrm{H}), 2.35(\mathrm{t}, J=6.0 \mathrm{~Hz}, 2 \mathrm{H}), 1.32(\mathrm{t}, J=7.0 \mathrm{~Hz}, 3 \mathrm{H})$; ${ }^{13} \mathrm{C} \mathrm{NMR}\left(100 \mathrm{MHz}, \mathrm{CDCl}_{3}\right): \delta 200.58,176.27,158.33,134.66,129.58,113.54,103.30,64.43,57.38$, 55.28, 34.26, 27.29, 14.23; HRMS (EI, [M] $]^{+}$) for $\mathrm{C}_{22} \mathrm{H}_{24} \mathrm{O}_{4}$ calcd. 352.1669, found: 352.1669.

\section{4,4'-Bis((tert-butyldimethylsilyl)oxy)-4'-ethoxy-5',6'-dihydro-2' $H$-[1,1':1',1' '-terphenyl]-2' -one} $(3 \mathbf{k})$

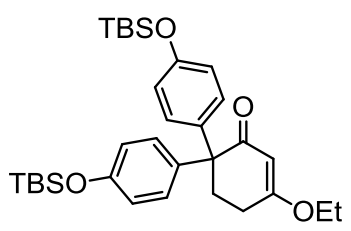

The reaction was conducted with $0.5 \mathrm{mmol}$ of $\mathbf{1 a}$ following the general procedure $\mathrm{A}$. The crude product was purified by flash column chromatography (hexanes/EtOAc $=10 / 1$ to $3 / 1)$ to afford $\mathbf{3 k}(130 \mathrm{mg}$, $47 \%$ ) as yellow solid (m.p. $120-121^{\circ} \mathrm{C}$ ). $\mathrm{R}_{f}: 0.5$ (hexanes/ EtOAc = 3/1). IR (cast): 2956, 2858, 1728, 1659, $1508 \mathrm{~cm}^{-1} ;{ }^{1} \mathbf{H}$ NMR $\left(400 \mathrm{MHz}, \mathrm{CDCl}_{3}\right): \delta 6.97(\mathrm{~d}, J=8.8 \mathrm{~Hz}, 4 \mathrm{H}), 6.74(\mathrm{~d}, J=8.8 \mathrm{~Hz}, 4 \mathrm{H})$, $5.51(\mathrm{~s}, 1 \mathrm{H}), 3.88(\mathrm{t}, J=7.0 \mathrm{~Hz}, 2 \mathrm{H}), 2.61(\mathrm{t}, J=6.0 \mathrm{~Hz}, 2 \mathrm{H}), 2.33(\mathrm{t}, J=6.0 \mathrm{~Hz}, 2 \mathrm{H}), 1.32(\mathrm{t}, J=7.0$ $\mathrm{Hz}, 3 \mathrm{H}), 0.97$ (s, 18H), 0.19 (s, 12H); ${ }^{13} \mathbf{C} \mathbf{N M R}\left(100 \mathrm{MHz}, \mathrm{CDCl}_{3}\right): \delta 200.70,176.31,154.39,135.30$, 129.61, 119.51, 103.46, 64.44, 57.56, 34.55, 27.32, 25.78, 18.29, 14.28, -4.24; HRMS (EI, [M] $]^{+}$) for $\mathrm{C}_{32} \mathrm{H}_{48} \mathrm{O}_{4} \mathrm{Si}_{2}$ calcd. 552.3086, found: 552.3084.

4,4'-Bis(benzyloxy)-4'-ethoxy-5',6'-dihydro-2' $H$-[1,1':1',1'-terphenyl]-2'-one (31)

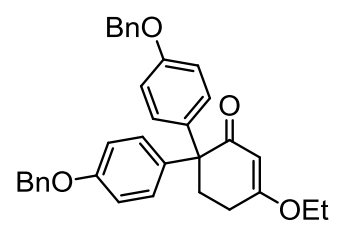


The reaction was conducted with $0.5 \mathrm{mmol}$ of $\mathbf{1 a}$ following the general procedure A. The crude product was purified by flash column chromatography (hexanes/EtOAc $=10 / 1$ to 3/1) to afford 31 (184 mg, 73 \%) as yellow solid (m.p. $\left.142-143{ }^{\circ} \mathrm{C}\right) . \mathrm{R}_{f}: 0.38$ (hexanes/ EtOAc = 3/1). IR (cast): 2909, 2869, 1652, 1608, $1509 \mathrm{~cm}^{-1} ;{ }^{1} \mathbf{H}$ NMR (400 MHz, $\left.\mathrm{CDCl}_{3}\right): \delta 7.46-7.30(\mathrm{~m}, 10 \mathrm{H}), 7.07(\mathrm{~d}, J=8.7 \mathrm{~Hz}, 4 \mathrm{H}), 6.92$ $(\mathrm{d}, J=8.8 \mathrm{~Hz}, 4 \mathrm{H}), 5.53(\mathrm{~s}, 1 \mathrm{H}), 5.04(\mathrm{~s}, 4 \mathrm{H}), 3.88(\mathrm{q}, J=7.0 \mathrm{~Hz}, 2 \mathrm{H}), 2.66(\mathrm{t}, J=5.9 \mathrm{~Hz}, 2 \mathrm{H}), 2.36$ $(\mathrm{t}, J=5.9 \mathrm{~Hz}, 2 \mathrm{H}), 1.33(\mathrm{t}, J=7.0 \mathrm{~Hz}, 3 \mathrm{H}) ;{ }^{13} \mathbf{C ~ N M R}\left(100 \mathrm{MHz}, \mathrm{CDCl}_{3}\right): \delta 200.52,176.28,157.69$, $137.18,134.95,129.66,128.69,128.07,127.66,114.44,103.37,70.11,64.45,57.45,34.29,27.32$, 14.26; HRMS (EI, [M] $]^{+}$) for $\mathrm{C}_{34} \mathrm{H}_{32} \mathrm{O}_{4}$ calcd. 504.2295, found: 504.2294.

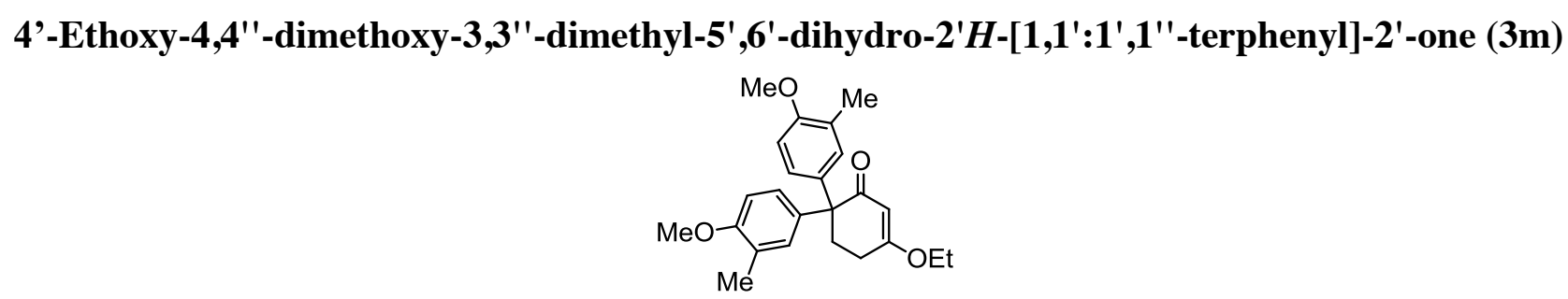

The reaction was conducted with $0.5 \mathrm{mmol}$ of $1 \mathrm{a}$ following the general procedure A. The crude product was purified by flash column chromatography (hexanes/EtOAc $=10 / 1$ to 3/1) to afford $\mathbf{3 m}(169 \mathrm{mg}$, $89 \%$ ) as brown oil. $\mathrm{R}_{f}: 0.35$ (hexanes/ EtOAc = 3/1). IR (film): 2946, 2833, 1651, 1605, $1502 \mathrm{~cm}^{-1}$; ${ }^{1} \mathbf{H}$ NMR $\left(400 \mathrm{MHz}, \mathrm{CDCl}_{3}\right): \delta 6.94(\mathrm{~d}, J=1.8 \mathrm{~Hz}, 2 \mathrm{H}), 6.90(\mathrm{dd}, J=8.5,2.4 \mathrm{~Hz}, 2 \mathrm{H}), 6.74(\mathrm{~d}, J=$ $8.5 \mathrm{~Hz}, 2 \mathrm{H}), 5.53(\mathrm{~s}, 1 \mathrm{H}), 3.87(\mathrm{q}, J=7.0 \mathrm{~Hz}, 2 \mathrm{H}), 3.81(\mathrm{~s}, 6 \mathrm{H}), 2.66(\mathrm{t}, J=6.0 \mathrm{~Hz}, 2 \mathrm{H}), 2.38(\mathrm{t}, J=$ $6.0 \mathrm{~Hz}, 2 \mathrm{H}), 2.18(\mathrm{~s}, 6 \mathrm{H}), 1.32(\mathrm{t}, J=7.0 \mathrm{~Hz}, 3 \mathrm{H}) ;{ }^{13} \mathbf{C} \mathbf{N M R}\left(100 \mathrm{MHz}, \mathrm{CDCl}_{3}\right): \delta 200.94,176.16$, 156.52 , 134.09, 130.73, 126.93, 126.08, 109.37, 103.36, 64.32, 57.42, 55.29, 34.37, 27.36, 16.56, 14.22; HRMS (EI, [M] $]^{+}$) for $\mathrm{C}_{24} \mathrm{H}_{28} \mathrm{O}_{4}$ calcd. 380.1982, found: 380.1980 .

\section{4'-Ethoxy-4,4"'-difluoro-3,3''-dimethoxy-5',6'-dihydro-2' $H$-[1,1':1',1'-terphenyl]-2'-one (3n)}

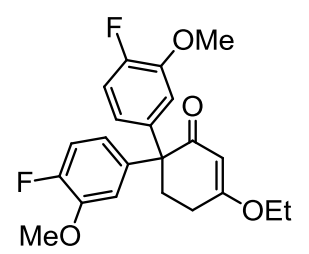

The reaction was conducted with $0.5 \mathrm{mmol}$ of $\mathbf{1 a}$ following the general procedure A. The crude product was purified by flash column chromatography (hexanes/EtOAc $=10 / 1$ to $3 / 1)$ to afford $\mathbf{3 n}(118 \mathrm{mg}$, $61 \%$ ) as yellow solid (m.p. $79-80{ }^{\circ} \mathrm{C}$ ). $\mathrm{R}_{f}: 0.2$ (hexanes/ EtOAc =3/1). IR (cast): 2981, 2940, 1652, 
1608, 1518, $1225 \mathrm{~cm}^{-1}{ }^{1}{ }^{1} \mathbf{H}$ NMR (400 MHz, $\left.\mathrm{CDCl}_{3}\right): \delta 6.99(\mathrm{dd}, J=10.9,8.6 \mathrm{~Hz}, 2 \mathrm{H}), 6.73(\mathrm{dd}, J=$ 8.1, 2.1 Hz, 2H), 6.66 (ddd, $J=8.3,3.9,2.4 \mathrm{~Hz}, 2 \mathrm{H}), 5.53$ (s, 1H), 3.89 (q, J=7.0 Hz, 2H), 3.77 (s, $6 \mathrm{H}), 2.64(\mathrm{t}, J=5.9 \mathrm{~Hz}, 2 \mathrm{H}), 2.39(\mathrm{t}, J=5.9 \mathrm{~Hz}, 2 \mathrm{H}), 1.33$ (t, $J=7.0 \mathrm{~Hz}, 3 \mathrm{H}) ;{ }^{13} \mathbf{C ~ N M R}(100 \mathrm{MHz}$, $\left.\mathrm{CDCl}_{3}\right): \delta 199.60,176.62,151.55(\mathrm{~d}, J=244.9 \mathrm{~Hz}), 147.30(\mathrm{~d}, J=10.6 \mathrm{~Hz}), 138.43(\mathrm{~d}, J=3.0 \mathrm{~Hz})$, $120.80(\mathrm{~d}, J=6.7 \mathrm{~Hz}), 115.51(\mathrm{~d}, J=18.1 \mathrm{~Hz}), 114.50$ (d, $J=1.6 \mathrm{~Hz}), 103.26,64.67,58.13,56.39$, 34.49, 27.25, 14.20; ${ }^{19}$ F NMR (375 MHz, $\left.\mathrm{CDCl}_{3}\right): \delta-137.83$ (ddd, $\left.J=11.9,8.6,4.2 \mathrm{~Hz}\right)$; HRMS (EI, $[\mathrm{M}]^{+}$) for $\mathrm{C}_{22} \mathrm{H}_{22} \mathrm{~F}_{2} \mathrm{O}_{4}$ calcd. 388.1481, found: 388.1479 .

\section{4'-Ethoxy-3,3'’-bis(trifluoromethyl)-5',6'-dihydro-2' $H$-[1,1':1',1'-terphenyl]-2'-one (3o)}

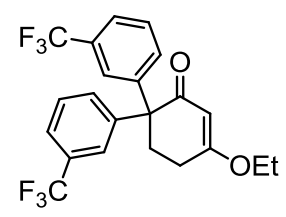

The reaction was conducted with $0.5 \mathrm{mmol}$ of $\mathbf{1 a}$ following the general procedure A. The crude product was purified by flash column chromatography (hexanes/EtOAc $=10 / 1$ to 3/1) to afford 30 (165 mg, $77 \%$ ) as yellow oil. $\mathrm{R}_{f}: 0.45$ (hexanes/ EtOAc = 3/1). IR (film): 2985, 2953, 1657, 1607, 1456, 1328 $\mathrm{cm}^{-1} ;{ }^{1} \mathbf{H}$ NMR $\left(400 \mathrm{MHz}, \mathrm{CDCl}_{3}\right): \delta 7.54(\mathrm{~d}, J=7.9 \mathrm{~Hz}, 2 \mathrm{H}), 7.46-7.40(\mathrm{~m}, 4 \mathrm{H}), 7.28(\mathrm{~d}, J=8.1 \mathrm{~Hz}$, 2H), $5.59(\mathrm{~s}, 1 \mathrm{H}), 3.92(\mathrm{q}, J=7.0 \mathrm{~Hz}, 2 \mathrm{H}), 2.76(\mathrm{t}, J=6.0 \mathrm{~Hz}, 2 \mathrm{H}), 2.36(\mathrm{t}, J=6.0 \mathrm{~Hz}, 2 \mathrm{H}), 1.35(\mathrm{t}, J$ $=7.0 \mathrm{~Hz}, 3 \mathrm{H}) ;{ }^{13} \mathbf{C} \mathbf{N M R}\left(100 \mathrm{MHz}, \mathrm{CDCl}_{3}\right): \delta 198.14,176.71,142.87,132.48,130.8(\mathrm{q}, J=32.0 \mathrm{~Hz})$, $128.91,124.82$ (q, $J=3.8 \mathrm{~Hz}), 124.30$ (q, $J=3.7 \mathrm{~Hz}), 124.18(\mathrm{q}, J=271.1 \mathrm{~Hz}), 103.31,64.88,58.36$, 33.77, 27.06, 14.19; ${ }^{19}$ F NMR (375 MHz, $\left.\mathrm{CDCl}_{3}\right)$ : -63.82; HRMS (EI, [M] ${ }^{+}$) for $\mathrm{C}_{22} \mathrm{H}_{18} \mathrm{~F}_{6} \mathrm{O}_{2}$ calcd. 428.1206, found: 428.1205 .

4'-Ethoxy-4,4'’-bis(trifluoromethyl)-5',6'-dihydro-2' H-[1,1':1',1'-terphenyl]-2' -one (3p)

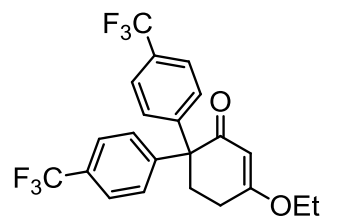

The reaction was conducted with $0.5 \mathrm{mmol}$ of $\mathbf{1 a}$ following the general procedure A. The crude product was purified by flash column chromatography (hexanes/EtOAc $=20 / 1$ to 6/1) to afford 3p $(147 \mathrm{mg}$, $69 \%$ ) as yellow solid (m.p. 109-110 ${ }^{\circ} \mathrm{C}$ ). $\mathrm{R}_{f}: 0.25$ (hexanes/ EtOAc = 6/1). IR (cast): 2942, 1651, 1602, $1320 \mathrm{~cm}^{-1} ;{ }^{1} \mathbf{H}$ NMR (400 MHz, $\left.\mathrm{CDCl}_{3}\right): \delta 7.57$ (d, $\left.J=8.3 \mathrm{~Hz}, 4 \mathrm{H}\right), 7.26(\mathrm{~d}, J=8.1 \mathrm{~Hz}, 4 \mathrm{H})$, 
$5.58(\mathrm{~s}, 1 \mathrm{H}), 3.91(\mathrm{q}, J=7.0 \mathrm{~Hz}, 2 \mathrm{H}), 2.76(\mathrm{t}, J=6.0 \mathrm{~Hz}, 2 \mathrm{H}), 2.36(\mathrm{t}, J=6.0 \mathrm{~Hz}, 2 \mathrm{H}), 1.35(\mathrm{t}, J=7.0$ $\mathrm{Hz}, 3 \mathrm{H}) ;{ }^{13} \mathrm{C}$ NMR (100 MHz, $\left.\mathrm{CDCl}_{3}\right): \delta 198.22,176.80,145.87,129.53$ (q, $\left.J=32.4 \mathrm{~Hz}\right), 129.01$, $125.35(\mathrm{q}, J=3.7 \mathrm{~Hz}), 124.17(\mathrm{q}, J=270.7 \mathrm{~Hz}), 103.30,64.89,58.51,33.72,27.05,14.21 ;{ }^{19} \mathbf{F}$ NMR (375 MHz, $\mathrm{CDCl}_{3}$ ): -63.82; HRMS (EI, [M] $]^{+}$) for $\mathrm{C}_{22} \mathrm{H}_{18} \mathrm{~F}_{6} \mathrm{O}_{2}$ calcd. 428.1206, found: 428.1205.

\section{Di-tert-butyl 3,3'-(4-ethoxy-2-oxocyclohex-3-ene-1,1-diyl)bis(9H-carbazole-9-carboxylate) (3q)}

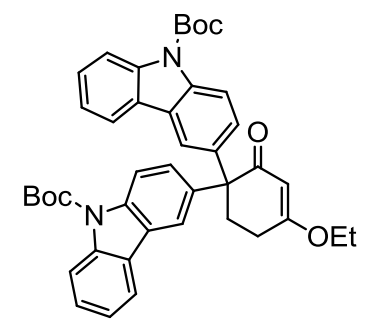

The reaction was conducted with $0.5 \mathrm{mmol}$ of $1 \mathrm{a}$ following the general procedure A. The crude product was purified by flash column chromatography (hexanes/EtOAc $=10 / 1$ to 3/1) to afford $\mathbf{3 q}(252 \mathrm{mg}$, $75 \%$ ) as light brown solid (m.p. $162-163{ }^{\circ} \mathrm{C}$ ). $\mathrm{R}_{f}: 0.3$ (hexanes/ EtOAc $=3 / 1$ ). IR (cast): 2979, 1726, 1652, 1608, $1359 \mathrm{~cm}^{-1} ;{ }^{1} \mathbf{H}$ NMR (400 MHz, $\left.\mathrm{CDCl}_{3}\right): \delta 8.31(\mathrm{~d}, J=8.4 \mathrm{~Hz}, 2 \mathrm{H}), 8.22(\mathrm{~d}, J=8.8 \mathrm{~Hz}$, 2H), $7.88(\mathrm{~d}, J=7.7 \mathrm{~Hz}, 2 \mathrm{H}), 7.81(\mathrm{~s}, 2 \mathrm{H}), 7.46-7.42(\mathrm{~m}, 2 \mathrm{H}), 7.34-7.28(\mathrm{~m}, 4 \mathrm{H}), 5.66(\mathrm{~s}, 1 \mathrm{H}), 3.92$ $(\mathrm{q}, J=7.0 \mathrm{~Hz}, 2 \mathrm{H}), 2.96(\mathrm{t}, J=5.7 \mathrm{~Hz}, 2 \mathrm{H}), 2.46(\mathrm{t}, J=5.7 \mathrm{~Hz}, 2 \mathrm{H}), 1.74(\mathrm{~s}, 18 \mathrm{H}), 1.33(\mathrm{t}, J=7.0 \mathrm{~Hz}$, 3H); ${ }^{13}$ C NMR (100 MHz, $\left.\mathrm{CDCl}_{3}\right): \delta 200.45,176.57,151.21,139.02,137.54,137.43,128.00,127.20$, $125.87,125.80,123.07,119.83,119.65,116.36,116.06,103.56,84.02,64.60,58.55,34.78,28.51$, 27.50, 14.27; HRMS (FD, [M] $]^{+}$) for $\mathrm{C}_{42} \mathrm{H}_{42} \mathrm{~N}_{2} \mathrm{O}_{6}$ calcd. 670.3037, found: 670.3055.

\section{6,6-Bis(9-(tert-butyldimethylsilyl)-9H-carbazol-3-yl)-3-ethoxycyclohex-2-en-1-one (3r)}

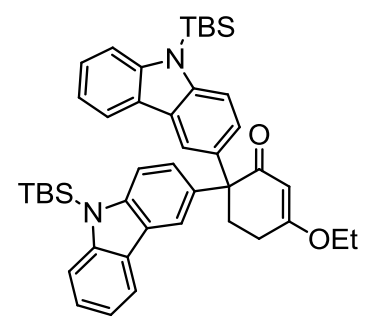

The reaction was conducted with $0.5 \mathrm{mmol}$ of $\mathbf{1 a}$ following the general procedure $\mathrm{A}$. The crude product was purified by flash column chromatography (hexanes/EtOAc $=10 / 1$ to $3 / 1)$ to afford $3 r$ ( $237 \mathrm{mg}$, $68 \%$ ) as yellow solid (m.p. $147-148^{\circ} \mathrm{C}$ ). $\mathrm{R}_{f}: 0.5$ (hexanes/ EtOAc $=3 / 1$ ). IR (cast): 2952, 2856, 1651, 1608, $1478 \mathrm{~cm}^{-1} ;{ }^{1} \mathbf{H}$ NMR (400 MHz, $\left.\mathrm{CDCl}_{3}\right): \delta 7.94(\mathrm{~d}, J=7.5 \mathrm{~Hz}, 2 \mathrm{H}), 7.89(\mathrm{~s}, 2 \mathrm{H}), 7.57(\mathrm{~d}, J=$ 
$8.4 \mathrm{~Hz}, 2 \mathrm{H}), 7.51(\mathrm{~d}, J=8.8 \mathrm{~Hz}, 2 \mathrm{H}), 7.36-7.29(\mathrm{~m}, 2 \mathrm{H}), 7.24-7.14(\mathrm{~m}, 4 \mathrm{H}), 5.66(\mathrm{~s}, 1 \mathrm{H}), 3.91(\mathrm{q}, J=$ $7.0 \mathrm{~Hz}, 2 \mathrm{H}), 2.95(\mathrm{t}, J=5.8 \mathrm{~Hz}, 2 \mathrm{H}), 2.48(\mathrm{t}, J=5.8 \mathrm{~Hz}, 2 \mathrm{H}), 1.32(\mathrm{t}, J=7.0 \mathrm{~Hz}, 3 \mathrm{H}), 1.05(\mathrm{~s}, 18 \mathrm{H})$, $0.73(\mathrm{~s}, 12 \mathrm{H}) ;{ }^{13} \mathrm{C} \mathrm{NMR}\left(100 \mathrm{MHz}, \mathrm{CDCl}_{3}\right): \delta 201.39,176.39,145.50,144.02,134.14,126.57,126.51$, $126.16,125.20$, 120.01, 119.74, 119.57, 114.17, 113.73, 103.68, 64.42, 58.51, 35.21, 27.67, 26.73, 20.68, 14.30, -1.20; HRMS (EI, [M] ${ }^{+}$) for $\mathrm{C}_{44} \mathrm{H}_{54} \mathrm{~N}_{2} \mathrm{O}_{2} \mathrm{Si}_{2}$ calcd. 698.3718, found: 698.3714.

\section{3-Ethoxy-6,6-bis(1-methyl-1H-indol-5-yl)cyclohex-2-en-1-one (3s)}

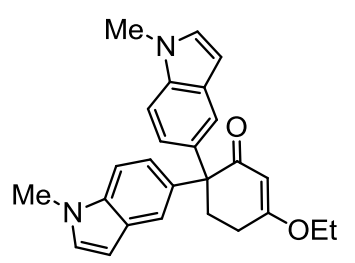

The reaction was conducted with $0.5 \mathrm{mmol}$ of $\mathbf{1 a}$ following the general procedure $\mathrm{A}$. The crude product was purified by flash column chromatography (hexanes/EtOAc $=10 / 1$ to 3/1) to afford 3s (97 mg, 49 \%) as light yellow solid (m.p. 213-214 ${ }^{\circ} \mathrm{C}$ ). $\mathrm{R}_{f}: 0.15$ (hexanes/ EtOAc = 3/1). IR (cast): 2951, 2924, 1647, 1607, 1511, $1185 \mathrm{~cm}^{-1}$; ${ }^{1} \mathbf{H}$ NMR (400 MHz, $\left.\mathrm{CDCl}_{3}\right): \delta$ 7.42-7.37 (d, 2H), 7.25 (d, J = 9.8 Hz, 2H), $7.10(\mathrm{dd}, J=8.7,1.6 \mathrm{~Hz}, 2 \mathrm{H}), 7.00(\mathrm{~d}, J=3.0 \mathrm{~Hz}, 2 \mathrm{H}), 6.39$ (d, $J=2.9 \mathrm{~Hz}, 2 \mathrm{H}), 5.59(\mathrm{~s}, 1 \mathrm{H})$, $3.86(\mathrm{q}, J=7.0 \mathrm{~Hz}, 2 \mathrm{H}), 3.77(\mathrm{~s}, 6 \mathrm{H}), 2.86(\mathrm{t}, J=5.9 \mathrm{~Hz}, 2 \mathrm{H}), 2.42(\mathrm{t}, J=5.9 \mathrm{~Hz}, 2 \mathrm{H}), 1.30(\mathrm{t}, J=7.0$ $\mathrm{Hz}, 3 \mathrm{H}) ;{ }^{13} \mathbf{C}$ NMR $\left(100 \mathrm{MHz}, \mathrm{CDCl}_{3}\right): \delta 201.84,176.13,135.67,134.06,128.91,128.18,123.10$, $120.88,108.87,103.69,101.37,64.28,58.73,35.10,32.97,27.67,14.30 ;$ HRMS (EI, [M] ${ }^{+}$) for $\mathrm{C}_{26} \mathrm{H}_{26} \mathrm{~N}_{2} \mathrm{O}_{2}$ calcd. 398.1989, found: 398.1993.

\section{3-Ethoxy-5,5-di-p-tolylcyclopent-2-en-1-one (3t)}

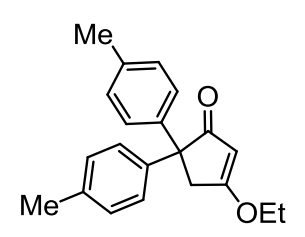

The reaction was conducted with $0.5 \mathrm{mmol}$ of $\mathbf{1 b}$ following the general procedure A. The crude product was purified by flash column chromatography (hexanes/EtOAc $=10 / 1$ to 3/1) to afford $\mathbf{3 t}$ (79 $\mathrm{mg}, 52$ \%) as brown oil. $\mathrm{R}_{f}: 0.4$ (hexanes/ EtOAc $=3 / 1$ ). IR (film): 2982, 2922, 1697, 1599, $1512 \mathrm{~cm}^{-1} ;{ }^{1} \mathbf{H}$ NMR (400 MHz, $\left.\mathrm{CDCl}_{3}\right): \delta 7.16(\mathrm{~d}, J=8.2 \mathrm{~Hz}, 4 \mathrm{H}), 7.11(\mathrm{~d}, J=8.2 \mathrm{~Hz}, 4 \mathrm{H}), 5.35(\mathrm{~s}, 1 \mathrm{H}), 4.11(\mathrm{q}, J$ $=7.1 \mathrm{~Hz}, 2 \mathrm{H}), 3.38(\mathrm{~s}, 2 \mathrm{H}), 2.32(\mathrm{~s}, 6 \mathrm{H}), 1.44(\mathrm{t}, J=7.1 \mathrm{~Hz}, 3 \mathrm{H}) ;{ }^{13} \mathbf{C} \mathbf{N M R}\left(100 \mathrm{MHz}, \mathrm{CDCl}_{3}\right): \delta$ 205.49, 187.50, 140.90, 136.35, 129.14, 127.91, 103.19, 67.93, 60.31, 46.77, 21.06, 14.29; HRMS (EI, 
$[\mathrm{M}]^{+}$) for $\mathrm{C}_{21} \mathrm{H}_{22} \mathrm{O}_{2}$ calcd. 306.1614, found: 306.1615 .

\section{4-Ethoxy-3,4'-dimethyl-5,6-dihydro-[1,1'-biphenyl]-2(1H)-one (2c)}

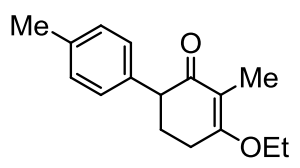

A round-bottom flask containing a vinylogous ester $1 \mathrm{c}(0.5 \mathrm{mmol}), 4$-bromo-toluene (6.0 equiv, $513 \mathrm{mg}), \mathrm{Pd}(\mathrm{OAc})_{2}(10 \mathrm{~mol} \%, 11 \mathrm{mg})$ and $\mathrm{PAd}_{3}(12 \mathrm{~mol} \%, 26 \mathrm{mg})$ was vacuumed for 2 minutes. The whole system was backfilled with $\mathrm{N}_{2}$, and then $5 \mathrm{~mL}$ of toluene was added to the flask. To the stirring mixture was added a solution of LiHMDS ( $1 \mathrm{M}, 6.0$ equiv, $4.8 \mathrm{~mL}$ ) over 5 minutes at room temperature. The resulting mixture was then stirred at room temperature for 30 minutes. After completion of the reaction as indicated by TLC, a saturated aqueous solution of $\mathrm{NH}_{4} \mathrm{Cl}$ was added to quench the reaction at room temperature. The reaction mixture was filtered through celite. The filtrate was diluted with ethyl acetate and extracted with water. The organic layers were dried over anhydrous $\mathrm{MgSO}_{4}$, filtered and concentrated under reduced pressure. The crude residue thus obtained was purified by flash column chromatography (hexanes/EtOAc $=10 / 1$ to $3 / 1)$ to afford $2 \mathrm{c}(93 \mathrm{mg}, 76 \%)$ as yellow solid (m.p. $98-99^{\circ} \mathrm{C}$ ). $\mathrm{R}_{f}$ : 0.4 (hexanes/ EtOAc =3/1). IR (cast): 2952, 2923, 1644, 1615, $1378 \mathrm{~cm}^{-1} ;{ }^{1} \mathbf{H}$ NMR $\left(400 \mathrm{MHz}, \mathrm{CDCl}_{3}\right): \delta 7.12(\mathrm{~d}, J=7.9 \mathrm{~Hz}, 2 \mathrm{H}), 7.03(\mathrm{~d}, J=7.9 \mathrm{~Hz}, 2 \mathrm{H}), 4.07$ (q, $J=7.0 \mathrm{~Hz}, 2 \mathrm{H}), 3.49(\mathrm{dd}, J=8.9,4.9 \mathrm{~Hz}, 1 \mathrm{H}), 2.59(\mathrm{t}, J=5.7 \mathrm{~Hz}, 2 \mathrm{H}), 2.32(\mathrm{~s}, 3 \mathrm{H}), 2.30-2.15(\mathrm{~m}$, 2H), $1.78(\mathrm{~s}, 3 \mathrm{H}), 1.36(\mathrm{t}, J=7.0 \mathrm{~Hz}, 3 \mathrm{H}) ;{ }^{13} \mathbf{C ~ N M R}\left(100 \mathrm{MHz}, \mathrm{CDCl}_{3}\right): \delta 198.75,170.85,137.18$, $136.31,129.25,128.17,115.53,63.58,50.78,28.88,24.20,21.18,15.47,7.94$; HRMS (EI, [M] $]^{+}$) for $\mathrm{C}_{16} \mathrm{H}_{20} \mathrm{O}_{2}$ calcd. 244.1458, found: 244.1456.

\section{General Procedure B for the Cascade Triarylation (Scheme 4 and Scheme 5).}

A round-bottom flask containing a vinylogous ester $(0.5 \mathrm{mmol})$, an aryl bromide $(6.0$ equiv), $\mathrm{Pd}(\mathrm{OAc})_{2}(10 \mathrm{~mol} \%, 11 \mathrm{mg})$ and $\mathrm{PAd}_{3}(12 \mathrm{~mol} \%, 26 \mathrm{mg})$ was vacuumed for 2 minutes. The whole system was backfilled with $\mathrm{N}_{2}$, and then $5 \mathrm{~mL}$ of toluene was added to the flask. To the stirring mixture was added a solution of LiHMDS ( $1 \mathrm{M}, 6.0$ equiv, $4.8 \mathrm{~mL})$ over 5 minutes at room temperature. The resulting mixture was then stirred at $70{ }^{\circ} \mathrm{C}$ (temperature of the aluminum heating block) for 30 minutes. After completion of the reaction as indicated by TLC, a saturated aqueous solution of $\mathrm{NH}_{4} \mathrm{Cl}$ was 
added to quench the reaction at room temperature. The reaction mixture was filtered through celite. The filtrate was diluted with ethyl acetate and extracted with water. The organic layers were dried over anhydrous $\mathrm{MgSO}_{4}$, filtered and concentrated under reduced pressure. The crude residue thus obtained was purified by flash column chromatography.

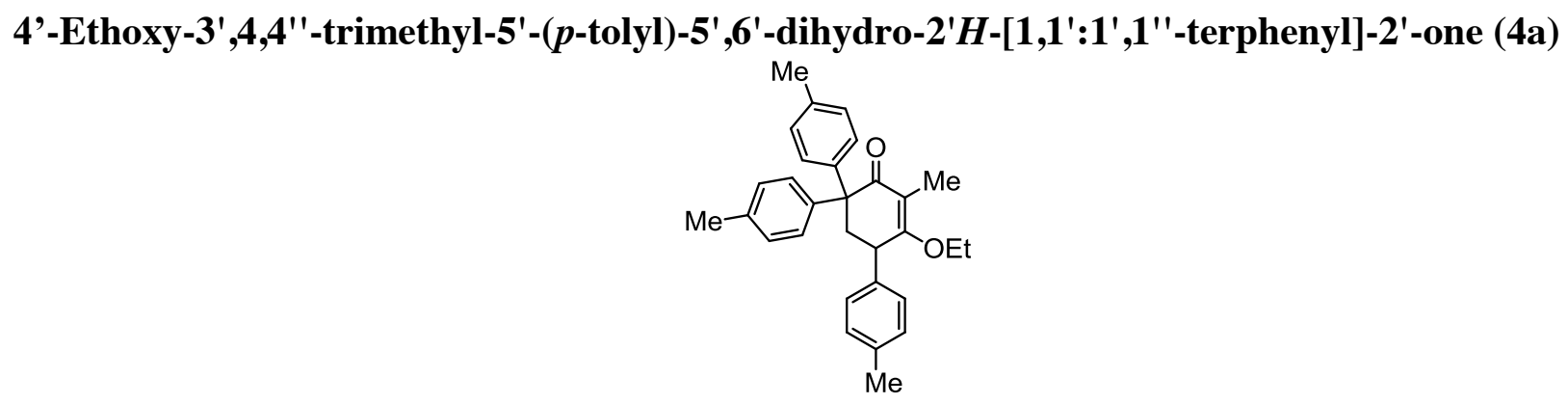

The reaction was conducted with $0.5 \mathrm{mmol}$ of $\mathbf{1 c}$ following the general procedure $\mathrm{B}$. The crude product was purified by flash column chromatography (hexanes/EtOAc $=20 / 1$ to $3 / 1$ ) to afford $4 \mathbf{a}$ (166 mg, $78 \%$ ) as yellow oil. $\mathrm{R}_{f}: 0.63$ (hexanes/ EtOAc = 3/1). IR (film): 3021, 2935, 1658, 1620, $1512 \mathrm{~cm}^{-1}$; ${ }^{1}$ H NMR (400 MHz, $\left.\mathrm{CDCl}_{3}\right): \delta 7.21-7.03(\mathrm{~m}, 8 \mathrm{H}), 6.97(\mathrm{~d}, J=8.0 \mathrm{~Hz}, 2 \mathrm{H}), 6.83(\mathrm{~d}, J=7.8 \mathrm{~Hz}, 2 \mathrm{H})$, 3.61 (br s, 1H), 3.50-3.46 (m, 2H), 2.85 (dd, $J=13.7,4.6 \mathrm{~Hz}, 1 \mathrm{H}), 2.70(\mathrm{dd}, J=13.3,10.9 \mathrm{~Hz}, 1 \mathrm{H})$, $2.37(\mathrm{~s}, 3 \mathrm{H}), 2.34(\mathrm{~s}, 3 \mathrm{H}), 2.25(\mathrm{~s}, 3 \mathrm{H}), 1.94(\mathrm{~s}, 3 \mathrm{H}), 0.88(\mathrm{t}, J=7.0 \mathrm{~Hz}, 3 \mathrm{H}) ;{ }^{13} \mathbf{C} \mathbf{~ N M R}(100 \mathrm{MHz}$, $\left.\mathrm{CDCl}_{3}\right): \delta 199.90,169.82,139.21,138.64,136.68,136.55,135.93,129.73,129.19,128.62,128.37$, 127.96, 127.75, 120.44, 64.82, 57.79, 42.39, 21.22, 21.19, 21.03, 15.26, 9.53; HRMS (EI, [M] $]^{+}$) for $\mathrm{C}_{30} \mathrm{H}_{32} \mathrm{O}_{2}$ calcd. 424.2397, found: 424.2391 .

\section{4'-Ethoxy-3',4,4' '-trimethyl-5',6'-dihydro-2' $H$-[1,1':1',1'-terphenyl]-2' -one (3u) and (1'S,3'S)- 6'-ethoxy-4,4' ',5'-trimethyl-2',3'-dihydro-[1,1':3',1'-terphenyl]-4'(1'H)-one (5)}
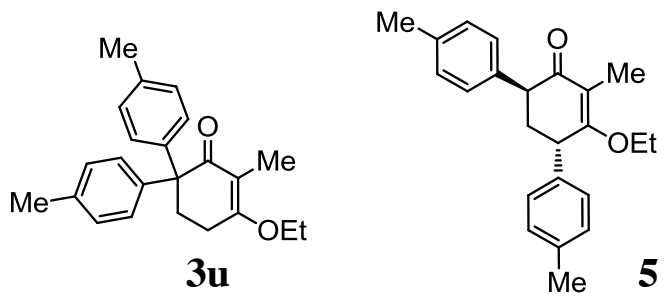

Along with the desired product $\mathbf{4 a}$, various amounts of compounds $\mathbf{3} \mathbf{u}$ and $\mathbf{5}$ were isolated in the triarylation reactions conducted with $0.5 \mathrm{mmol}$ of $\mathbf{1 c}$ (See Table 3 ). The crude product was purified by flash column chromatography (hexanes/EtOAc $=20 / 1$ to $3 / 1$ ) to afford an inseparable mixture of $\mathbf{3 z}$ 
and 5 (Table 3, entry 1; $109 \mathrm{mg}, 65 \%$ ) as yellow oil. $\mathrm{R}_{f}: 0.53$ (hexanes/ EtOAc $=3 / 1$ ). Spectral data (IR and HRMS) of the mixture is reported. IR (film): 2979, 2921, 1649, 1614, 1510, $1443 \mathrm{~cm}^{-1}$; HRMS (EI, [M] $]^{+}$) for $\mathrm{C}_{23} \mathrm{H}_{26} \mathrm{O}_{2}$ calcd. 334.1927, found: 334.1923 .

3u: ${ }^{1} \mathbf{H}$ NMR (400 MHz, $\mathrm{CDCl}_{3}$ ): $\delta$ 7.10-7.07 (m, 4H), 7.02-6.97 (m, 4H), 3.98-3.93 (q, $J=7.0 \mathrm{~Hz}$, 2H), 2.73-2.65 (m, 2H), $2.46(\mathrm{t}, J=5.3 \mathrm{~Hz}, 2 \mathrm{H}), 2.32(\mathrm{~s}, 6 \mathrm{H}), 1.81(\mathrm{~s}, 3 \mathrm{H}), 1.29(\mathrm{t}, J=7.0 \mathrm{~Hz}, 3 \mathrm{H})$; ${ }^{13} \mathrm{C}$ NMR $\left(100 \mathrm{MHz}, \mathrm{CDCl}_{3}\right): \delta$ 199.37,169.78, 115.24, 63.55, 57.11, 33.39, 23.57, 21.10, 15.32, 8.42 (Selected carbon signals are presented).

5: ${ }^{1}$ H NMR (400 MHz, $\left.\mathrm{CDCl}_{3}\right): \delta$ 7.20-7.15 (m, 4H), 7.10-7.07 (m, 2H), $6.93(\mathrm{~d}, J=8.0 \mathrm{~Hz}, 2 \mathrm{H}), 4.14$ (br s, 1H), 4.05-3.99 (m, 1H), 3.79-3.72 (m, 1H), $3.38(\mathrm{dd}, J=13.6,4.2 \mathrm{~Hz}, 1 \mathrm{H}), 2.71-2.69(\mathrm{~m} 1 \mathrm{H})$, $2.38(\mathrm{~s}, 3 \mathrm{H}), 2.30(\mathrm{~s}, 3 \mathrm{H}), 2.24-2.19(\mathrm{~m}, 1 \mathrm{H}), 1.90(\mathrm{~s}, 3 \mathrm{H}), 1.23(\mathrm{t}, J=7.0 \mathrm{~Hz}, 3 \mathrm{H}) ;{ }^{13} \mathrm{C}$ NMR (100 $\left.\mathrm{MHz}, \mathrm{CDCl}_{3}\right): \delta 198.70,170.16,116.91,63.51,46.84,40.84,38.68,21.20,15.40,8.26$ (Selected carbon signals are presented).

\section{4'-Ethoxy-3'-methyl-5'-phenyl-5',6'-dihydro-2'H-[1,1':1',1'-terphenyl]-2'-one (4b)}

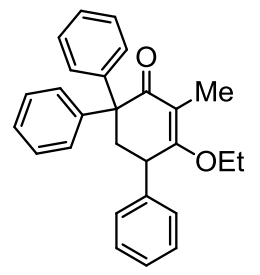

The reaction was conducted with $0.5 \mathrm{mmol}$ of $\mathbf{1 c}$ following the general procedure $\mathrm{B}$. The crude product was purified by flash column chromatography (hexanes/EtOAc $=20 / 1$ to $3 / 1)$ to afford $\mathbf{4 b}(112 \mathrm{mg}$, $58 \%$ ) as yellow oil. $\mathrm{R}_{f}: 0.625$ (hexanes/ EtOAc = 3/1). IR (film): 2905, 1652, 1602, $1470 \mathrm{~cm}^{-1} ;{ }^{1} \mathbf{H}$ NMR (400 MHz, $\left.\mathrm{CDCl}_{3}\right): \delta$ 7.41-7.27 (m, 5H), 7.26-7.22 (m, 3H), 7.21-7.11 (m, 5H), 6.97-6.93 (m, 2H), 3.66 (br s, 1H), 3.47 (q, $J=7.0 \mathrm{~Hz}, 2 \mathrm{H}), 2.94$ (dd, $J=13.8,4.9 \mathrm{~Hz}, 1 \mathrm{H}), 2.76$ (dd, $J=13.8,10.4$ $\mathrm{Hz}, 1 \mathrm{H}), 1.96(\mathrm{~s}, 3 \mathrm{H}), 0.87(\mathrm{t}, J=7.0 \mathrm{~Hz}, 3 \mathrm{H}) ;{ }^{13} \mathbf{C} \mathbf{N M R}\left(100 \mathrm{MHz}, \mathrm{CDCl}_{3}\right): \delta 199.54,169.61,144.04$, $142.05,141.48,129.11,128.80,128.55,128.16,127.88,127.68,127.14,126.53,120.67,64.88,58.44$ 44.61, 42.80, 15.22, 9.54 (A sp ${ }^{2}$ carbon signal is missing due to peak overlap); HRMS (EI, [M] $]^{+}$) for $\mathrm{C}_{27} \mathrm{H}_{26} \mathrm{O}_{2}$ calcd. 382.1926, found: 382.1922 . 
4'-Ethoxy-4,4' '-dimethoxy-5' -(4-methoxyphenyl)-3'-methyl-5',6'-dihydro-2' H-[1,1':1',1'"terphenyl]-2'-one (4c)

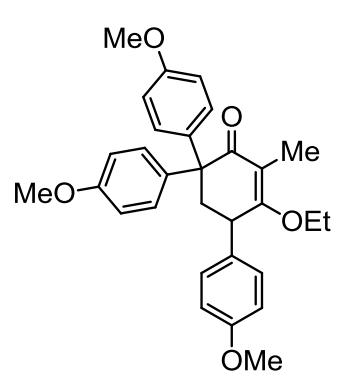

The reaction was conducted with $0.5 \mathrm{mmol}$ of $\mathbf{1 c}$ following the general procedure $\mathrm{B}$. The crude product was purified by flash column chromatography (hexanes/EtOAc $=10 / 1$ to $3 / 1)$ to afford $\mathbf{4 c}(111 \mathrm{mg}$, $47 \%$ ) as yellow oil. $\mathrm{R}_{f}: 0.5$ (hexanes/ EtOAc = 3/1). IR (film): 2930, 2832, 1651, 1607, $1508 \mathrm{~cm}^{-1}$; ${ }^{1} \mathbf{H}$ NMR $\left(400 \mathrm{MHz} \mathrm{CDCl}_{3}\right): \delta 7.11(\mathrm{dd}, J=15.6,8.4 \mathrm{~Hz}, 4 \mathrm{H}), 6.95-6.81(\mathrm{~m}, 6 \mathrm{H}), 6.71(\mathrm{~d}, J=8.6 \mathrm{~Hz}$, 2H), 3.83 (s, 3H), 3.80 (s, 3H), 3.73 (s, 3H), $3.60($ br s, $1 \mathrm{H}), 3.51-3.49(\mathrm{~m}, 2 \mathrm{H}), 2.85$ (dd, $J=13.7,4.7$ Hz, 1H), 2.69 (dd, $J=13.7,8 \mathrm{~Hz}, 1 \mathrm{H}), 1.95$ (s, 3H), 0.91 (t, $J=7.0 \mathrm{~Hz}, 3 \mathrm{H}) ;{ }^{13} \mathrm{C}$ NMR (100 MHz, $\left.\mathrm{CDCl}_{3}\right): \delta 200.04,169.93,158.57,158.40,157.99,136.60,134.01,133.80,129.70,129.07,128.86$, $120.19,114.42,113.78,113.01,64.75,57.10,55.37,55.31,55.22,44.93,41.92,15.25,9.46$; HRMS (EI, [M] $]^{+}$) for $\mathrm{C}_{30} \mathrm{H}_{32} \mathrm{O}_{5}$ calcd. 472.2244, found: 472.2249 .

\section{4'-Ethoxy-3'-methyl-4,4''-bis(trifluoromethyl)-5' -(4-(trifluoromethyl)phenyl)-5',6' -dihydro-} 2'H-[1,1':1',1''-terphenyl]-2'-one (4d)

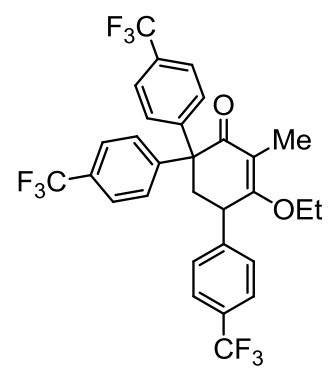

The reaction was conducted with $0.5 \mathrm{mmol}$ of $\mathbf{1 c}$ following the general procedure $\mathrm{B}$. The crude product was purified by flash column chromatography (hexanes/EtOAc $=20 / 1$ to $3 / 1)$ to afford $\mathbf{4 d}(115 \mathrm{mg}$, $49 \%$ ) as yellow solid (m.p. $54-55^{\circ} \mathrm{C}$ ). $\mathrm{R}_{f}: 0.63$ (hexanes/ EtOAc = 3/1). IR (cast): 2932, 1652, 1614, 1448, $1322 \mathrm{~cm}^{-1} ;{ }^{1} \mathbf{H}$ NMR (400 MHz, $\left.\mathrm{CDCl}_{3}\right): \delta 7.63(\mathrm{~d}, J=8.3 \mathrm{~Hz}, 2 \mathrm{H}), 7.52(\mathrm{~d}, J=8.1 \mathrm{~Hz}, 2 \mathrm{H})$, $7.37(\mathrm{~d}, J=8.3 \mathrm{~Hz}, 2 \mathrm{H}), 7.29(\mathrm{~d}, J=8.2 \mathrm{~Hz}, 2 \mathrm{H}), 7.22(\mathrm{~d}, J=8.0 \mathrm{~Hz}, 2 \mathrm{H}), 7.00(\mathrm{~d}, J=8.3 \mathrm{~Hz}, 2 \mathrm{H})$, 3.83 (br s, 1H), 3.65-3.44 (m, 2H), $3.08(\mathrm{dd}, J=13.9,5.2 \mathrm{~Hz}, 1 \mathrm{H}), 2.85(\mathrm{dd}, J=13.9,8.6 \mathrm{~Hz}, 1 \mathrm{H})$, $2.00(\mathrm{~s}, 3 \mathrm{H}), 0.99(\mathrm{t}, J=7.0 \mathrm{~Hz}, 3 \mathrm{H}) ;{ }^{13} \mathbf{C} \mathbf{N M R}\left(100 \mathrm{MHz}, \mathrm{CDCl}_{3}\right): \delta 197.53,168.92,146.20,145.58$, 
144.61, $129.84(\mathrm{q}, J=33.0 \mathrm{~Hz}), 129.79(\mathrm{q}, J=33.0 \mathrm{~Hz}), 129.32(\mathrm{q}, J=33.0 \mathrm{~Hz}), 129.09,128.57$, 128.15, $126.07(\mathrm{q}, J=3.7 \mathrm{~Hz}), 125.64(\mathrm{q}, J=3.7 \mathrm{~Hz}), 124.81$ (q, $J=3.7 \mathrm{~Hz}), 124.08(\mathrm{q}, J=271.0$ Hz), $124.05(\mathrm{q}, J=270.0 \mathrm{~Hz}), 124.00$ (q, $J=270.0 \mathrm{~Hz}), 120.45,65.16,57.82,43.06,41.96,15.22$, 9.43; ${ }^{19}$ F NMR $\left(375 \mathrm{MHz}, \mathrm{CDCl}_{3}\right)$ : -63.93, -64.00, -64.08; HRMS (EI, [M] ${ }^{+}$) for $\mathrm{C}_{30} \mathrm{H}_{23} \mathrm{~F}_{9} \mathrm{O}_{2}$ calcd. 586.1549, found: 586.1542 .

\section{3'-Butyl-4'-ethoxy-4,4' '-dimethyl-5' -(p-tolyl)-5',6'-dihydro-2' $H$-[1,1':1',1'-terphenyl]-2' -one} (4e)

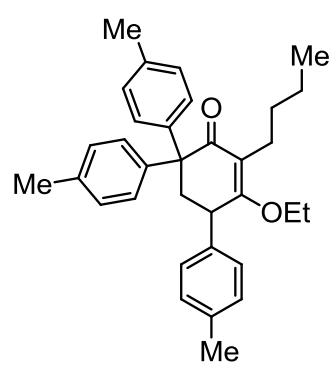

The reaction was conducted with $0.5 \mathrm{mmol}$ of $\mathbf{1 d}$ following the general procedure $\mathrm{B}$. The crude product was purified by flash column chromatography (hexanes/EtOAc $=20 / 1$ to $3 / 1)$ to afford $4 \mathbf{e}(170 \mathrm{mg}$, $73 \%$ ) as yellow solid (m.p. 82-83 ${ }^{\circ} \mathrm{C}$ ). $\mathrm{R}_{f}: 0.75$ (hexanes/ EtOAc = 3/1). IR (cast): 2953, 2921, 1657, 1611, $1512 \mathrm{~cm}^{-1} ;{ }^{1} \mathbf{H}$ NMR (400 MHz, $\left.\mathrm{CDCl}_{3}\right): \delta 7.12(\mathrm{t}, J=7.7 \mathrm{~Hz}, 6 \mathrm{H}), 7.07(\mathrm{~d}, J=8.0 \mathrm{~Hz}, 2 \mathrm{H})$, $6.97(\mathrm{~d}, J=8.1 \mathrm{~Hz}, 2 \mathrm{H}), 6.83(\mathrm{~d}, J=8.2 \mathrm{~Hz}, 2 \mathrm{H}), 3.60(\mathrm{dd}, J=10.3,4.7 \mathrm{~Hz}, 1 \mathrm{H}), 3.48-3.39(\mathrm{~m}, 2 \mathrm{H})$, $2.86(\mathrm{dd}, J=13.7,4.9 \mathrm{~Hz}, 1 \mathrm{H}), 2.68(\mathrm{dd}, J=13.7,10.5 \mathrm{~Hz}, 1 \mathrm{H}), 2.51-2.44(\mathrm{~m}, 2 \mathrm{H}), 2.36(\mathrm{~s}, 3 \mathrm{H}), 2.34$ (s, 3H), $2.24(\mathrm{~s}, 3 \mathrm{H}), 1.51-1.32(\mathrm{~m}, 4 \mathrm{H}), 0.94(\mathrm{t}, J=7.1 \mathrm{~Hz}, 3 \mathrm{H}), 0.88(\mathrm{t}, J=7.0 \mathrm{~Hz}, 3 \mathrm{H}) ;{ }^{13} \mathbf{C} \mathbf{N M R}$ $\left(100 \mathrm{MHz}, \mathrm{CDCl}_{3}\right): \delta 199.30,169.88,141.38,139.36,138.73,136.65,136.46,135.87,129.76,129.12$, $128.63,128.32,127.97,127.69,125.13,64.53,57.46,44.85,42.11,31.14,23.68,23.08,21.21,21.18$, 21.02, 15.21, 14.15; HRMS (EI, [M] ${ }^{+}$) for $\mathrm{C}_{33} \mathrm{H}_{38} \mathrm{O}_{2}$ calcd. 466.2853, found: 466.2851 .

\section{3'-benzyl-4'-ethoxy-4,4' '-dimethyl-5'-(p-tolyl)-5',6' -dihydro-2'H-[1,1':1',1'-terphenyl]-2' -one} (4f)

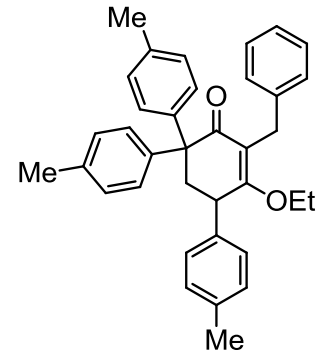


The reaction was conducted with $0.5 \mathrm{mmol}$ of $\mathbf{1 e}$ following the general procedure $\mathrm{B}$. The crude product was purified by flash column chromatography (hexanes/EtOAc $=20 / 1$ to 3/1) to afford $\mathbf{4 f}(141 \mathrm{mg}, 56$ \%) as yellow solid (m.p. 77-78 ${ }^{\circ} \mathrm{C}$ ). $\mathrm{R}_{f}: 0.63$ (hexanes/ EtOAc $=3 / 1$ ). IR (cast): 3024, 2922, 1655, 1610, $1511 \mathrm{~cm}^{-1} ;{ }^{1} \mathbf{H}$ NMR (400 MHz, $\left.\mathrm{CDCl}_{3}\right): \delta 7.34(\mathrm{~d}, J=7.2 \mathrm{~Hz}, 2 \mathrm{H}), 7.23(\mathrm{~d}, J=7.5 \mathrm{~Hz}, 2 \mathrm{H})$, 7.18-7.15 (m, 1H), 7.12 (d, J=7.8 Hz, 2H), 7.07-7.00 (m, 4H), 6.97-6.90 (m, 4H), 6.76 (d, $J=8.2 \mathrm{~Hz}$, 2H), $3.84(\mathrm{~s}, 2 \mathrm{H}), 3.66(\mathrm{dd}, J=10.0,4.8 \mathrm{~Hz}, 1 \mathrm{H}), 3.51-3.43(\mathrm{~m}, 2 \mathrm{H}), 2.87(\mathrm{dd}, J=13.9,4.9 \mathrm{~Hz}, 1 \mathrm{H})$, $2.68(\mathrm{dd}, J=13.9,10.2 \mathrm{~Hz}, 1 \mathrm{H}), 2.34(\mathrm{~s}, 6 \mathrm{H}), 2.22(\mathrm{~s}, 3 \mathrm{H}), 0.90(\mathrm{t}, J=7.0 \mathrm{~Hz}, 3 \mathrm{H}) ;{ }^{13} \mathbf{C}$ NMR $(100$ $\left.\mathrm{MHz}, \mathrm{CDCl}_{3}\right): \delta 198.87,170.24,141.60,141.18,139.18,138.30,136.75,136.46,135.93,129.85$, $129.36,129.10,128.57,128.37,128.04,128.03,127.63,125.57,123.71,64.74,57.38,44.79,42.14$, 29.78, 21.22, 21.18, 21.01, 15.22; HRMS (EI, [M] ${ }^{+}$) for $\mathrm{C}_{36} \mathrm{H}_{36} \mathrm{O}_{2}$ calcd. 500.2697, found: 500.2698.

\section{3-ethoxy-2-methyl-4,5,5-tri-p-tolylcyclopent-2-en-1-one (4g)}

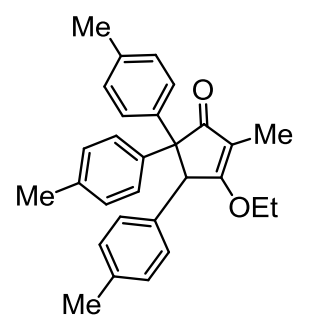

The reaction was conducted with $0.5 \mathrm{mmol}$ of $\mathbf{1 f}$ following the general procedure. The crude product was purified by flash column chromatography (hexanes/EtOAc $=20 / 1$ to $3 / 1)$ to afford $\mathbf{4 g}(129 \mathrm{mg}$, $63 \%$ ) as light yellow solid (m.p. $118-119^{\circ} \mathrm{C}$ ). $\mathrm{R}_{f}: 0.75$ (hexanes/ EtOAc =3/1). IR (cast): 2982, 2921, 1693, 1632, $1511 \mathrm{~cm}^{-1} ;{ }^{1} \mathbf{H}$ NMR (400 MHz, $\left.\mathrm{CDCl}_{3}\right): \delta 7.30(\mathrm{~d}, J=6.9 \mathrm{~Hz}, 2 \mathrm{H}), 7.10(\mathrm{~d}, J=7.9 \mathrm{~Hz}$, 2H), $6.79(\mathrm{~d}, J=7.7 \mathrm{~Hz}, 2 \mathrm{H}), 6.71-6.68(\mathrm{~m}, 6 \mathrm{H}), 4.88(\mathrm{~s}, 1 \mathrm{H}), 4.27-4.19(\mathrm{~m}, 1 \mathrm{H}), 4.04-3.95(\mathrm{~m}, 1 \mathrm{H})$, $2.32(\mathrm{~s}, 3 \mathrm{H}), 2.15(\mathrm{~s}, 3 \mathrm{H}), 2.11(\mathrm{~s}, 3 \mathrm{H}), 1.82(\mathrm{~s}, 3 \mathrm{H}), 1.24(\mathrm{t}, J=7.0 \mathrm{~Hz}, 3 \mathrm{H}) ;{ }^{13} \mathbf{C}$ NMR $(100 \mathrm{MHz}$, $\left.\mathrm{CDCl}_{3}\right): \delta 205.06,182.08,141.17,138.53,136.44,136.37,135.08,134.88,130.61,129.35,128.92$, 128.81, 127.91, 127.78, 115.25, 67.16, 65.74, 56.79, 21.07, 21.04, 20.98, 15.41, 7.01; HRMS (EI, $[\mathrm{M}]^{+}$) for $\mathrm{C}_{29} \mathrm{H}_{30} \mathrm{O}_{2}$ calcd. 410.2240, found: 410.2234 . 


\section{General Procedure C for the One-pot Sequential Diarylation (Scheme 6).}

A round-bottom flask containing a vinylogous ester $(0.5 \mathrm{mmol})$, an aryl bromide (1.1 equiv), $\mathrm{Pd}(\mathrm{OAc})_{2}(10 \mathrm{~mol} \%, 11 \mathrm{mg})$ and $\mathrm{PAd}_{3}(12 \mathrm{~mol} \%, 26 \mathrm{mg})$ was vacuumed for 2 minutes. The whole system was backfilled with $\mathrm{N}_{2}$, and then $5 \mathrm{~mL}$ of toluene was added to the flask. To the stirring mixture was added a solution of LiHMDS (1 M, 3.0 equiv, $2.4 \mathrm{~mL})$ over 3 minutes at room temperature. The resulting mixture was then stirred at room temperature for 15 minutes. After completion of the first arylation as indicated by TLC, another aryl bromide (1.5 equiv) was added and then the reaction temperature was raised to $70^{\circ} \mathrm{C}$ (temperature of the aluminum heating block). After stirring for another 15 minutes, a saturated aqueous solution of $\mathrm{NH}_{4} \mathrm{Cl}$ was added to quench the reaction at room temperature. The reaction mixture was filtered through celite. The filtrate was diluted with ethyl acetate and extracted with water. The organic layers were dried over anhydrous $\mathrm{MgSO}_{4}$, filtered and concentrated under reduced pressure. The crude residue thus obtained was purified by flash column chromatography.

\section{4'-Ethoxy-3,4-dimethoxy-5',6'-dihydro-2' $H$-[1,1':1',1'-terphenyl]-2'-one (3b)}

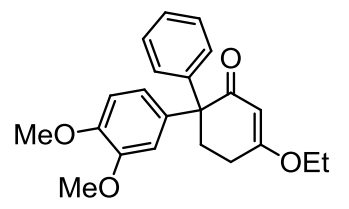

The reaction was conducted with $0.5 \mathrm{mmol}$ of $1 \mathrm{a}$ following the general procedure $\mathrm{C}$ (bromobenzene then 4-bromoveratrole). The crude product was purified by flash column chromatography (hexanes/EtOAc $=8 / 1$ to $3 / 1)$ to afford $\mathbf{3 b}(125 \mathrm{mg}, 71 \%)$ as yellow oil. $\mathrm{R}_{f}: 0.4$ (hexanes/ EtOAc $=$ 1/1). IR (film): 3058, 2953, 2835, 1654, 1609, $1517 \mathrm{~cm}^{-1} ;{ }^{1} \mathbf{H}$ NMR (400 MHz, $\left.\mathrm{CDCl}_{3}\right): \delta$ 7.31-7.11 $(\mathrm{m}, 3 \mathrm{H}), 7.12(\mathrm{~d}, J=7.3 \mathrm{~Hz}, 2 \mathrm{H}), 6.81(\mathrm{~d}, J=8.1 \mathrm{~Hz}, 1 \mathrm{H}), 6.71(\mathrm{~d}, J=8.8 \mathrm{~Hz}, 2 \mathrm{H}), 5.54(\mathrm{~s}, 1 \mathrm{H}), 3.90$ $(\mathrm{d}, J=7.0 \mathrm{~Hz}, 2 \mathrm{H}), 3.86(\mathrm{~s}, 3 \mathrm{H}), 3.76(\mathrm{~s}, 3 \mathrm{H}), 2.69(\mathrm{t}, J=5.8 \mathrm{~Hz}, 2 \mathrm{H}), 2.46-2.32(\mathrm{~m}, 2 \mathrm{H}), 1.32(\mathrm{t}, J=$ $7.0 \mathrm{~Hz}, 3 \mathrm{H}) ;{ }^{13} \mathrm{C}$ NMR $\left(100 \mathrm{MHz}, \mathrm{CDCl}_{3}\right): \delta 200.33,176.38,148.69,148.04,143.07,134.27,128.58$, $128.19,126.89,120.78,112.43,110.63,103.45,64.50,58.38,55.96,55.91,34.35,27.34,14.26$; HRMS (EI, [M] $]^{+}$) for $\mathrm{C}_{22} \mathrm{H}_{24} \mathrm{O}_{4}$ calcd. 352.1669, found: 352.1673 . 
4'-Ethoxy-3,5-difluoro-4' '-methyl-5',6'-dihydro-2' $H$-[1,1':1',1'-terphenyl]-2'-one (3v)

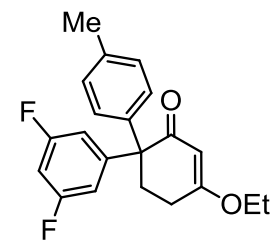

The reaction was conducted with $0.5 \mathrm{mmol}$ of $\mathbf{1 a}$ following the general procedure $\mathrm{C}$ (4-bromotoluene then 1-bromo-3,5-difluorobenzene). The crude product was purified by flash column chromatography (hexanes/EtOAc $=10 / 1$ to $3 / 1)$ to afford 3v $(142 \mathrm{mg}, 83 \%)$ as brown oil. $\mathrm{R}_{f}: 0.4$ (hexanes/ EtOAc $=$ 3/1). IR (film): 2982, 2926, 1656, 1608, 1513, $1188 \mathrm{~cm}^{-1} ;{ }^{\mathbf{1}} \mathbf{H} \mathbf{~ N M R}\left(400 \mathrm{MHz}, \mathrm{CDCl}_{3}\right): \delta 7.15$ (d, $J$ $=8.1 \mathrm{~Hz}, 2 \mathrm{H}), 7.07(\mathrm{~d}, J=8.3 \mathrm{~Hz}, 2 \mathrm{H}), 6.70-6.60(\mathrm{~m}, 3 \mathrm{H}), 5.53(\mathrm{~s}, 1 \mathrm{H}), 3.93-3.84(\mathrm{~m}, 2 \mathrm{H}), 2.72-2.59$ $(\mathrm{m}, 2 \mathrm{H}), 2.41-2.36(\mathrm{~m}, 2 \mathrm{H}), 2.34(\mathrm{~s}, 3 \mathrm{H}), 1.33(\mathrm{t}, J=7.0 \mathrm{~Hz}, 3 \mathrm{H}) ;{ }^{13} \mathbf{C} \mathbf{N M R}\left(100 \mathrm{MHz}, \mathrm{CDCl}_{3}\right): \delta$ 198.88, 176.57, $162.73(\mathrm{dd}, J=246.0,13.0 \mathrm{~Hz}), 147.84(\mathrm{t}, J=8.0 \mathrm{~Hz}), 137.24,137.10,129.34,128.21$, $111.97(\mathrm{dd}, J=18.0,7.0 \mathrm{~Hz}), 103.18,102.32(\mathrm{t}, J=26.0 \mathrm{~Hz}), 64.66,58.26,33.85,27.13,21.10,14.21$; ${ }^{19}$ F NMR (375 MHz, $\left.\mathrm{CDCl}_{3}\right)$ : -111.67 (t, $J=8.5 \mathrm{~Hz}$ ); HRMS (EI, [M] ${ }^{+}$) for $\mathrm{C}_{21} \mathrm{H}_{20} \mathrm{~F}_{2} \mathrm{O}_{2}$ calcd. 342.1426, found: 342.1424 .

\section{1-(Benzo[d][1,3]dioxol-5-yl)-4-ethoxy-4'-methoxy-5,6-dihydro-[1,1'-biphenyl]-2(1H)-one (3w)}

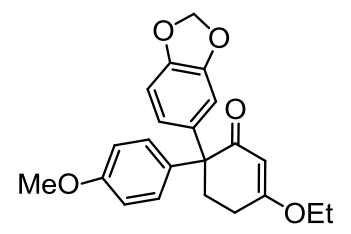

The reaction was conducted with $0.5 \mathrm{mmol}$ of $1 \mathrm{a}$ following the general procedure $\mathrm{C}$ (1-bromo-3,4(methylenedioxy)benzene then 4-bromoanisole). The crude product was purified by flash column chromatography (hexanes/EtOAc $=8 / 1$ to $3 / 1)$ to afford $\mathbf{3 w}(95 \mathrm{mg}, 52 \%)$ as yellow oil. $\mathrm{R}_{f}: 0.63$ (hexanes/ EtOAc = 1/1). IR (film): 2954, 2837, 1653, 1608, $1510 \mathrm{~cm}^{-1} ;{ }^{1} \mathbf{H} \mathbf{~ N M R}\left(400 \mathrm{MHz}, \mathrm{CDCl}_{3}\right)$ : $\delta 7.08(\mathrm{~d}, J=8.9 \mathrm{~Hz}, 2 \mathrm{H}), 6.84(\mathrm{~d}, J=8.9 \mathrm{~Hz}, 2 \mathrm{H}), 6.72(\mathrm{~d}, J=8.2 \mathrm{~Hz}, 1 \mathrm{H}), 6.64-6.55(\mathrm{~m}, 2 \mathrm{H}), 5.92$ $(\mathrm{s}, 2 \mathrm{H}), 5.51(\mathrm{~s}, 1 \mathrm{H}), 3.87$ (q, $J=6.2 \mathrm{~Hz}, 2 \mathrm{H}), 3.79(\mathrm{~s}, 3 \mathrm{H}), 2.69-2.57(\mathrm{~m}, 2 \mathrm{H}), 2.36(\mathrm{t}, J=5.9 \mathrm{~Hz}, 2 \mathrm{H})$, $1.32(\mathrm{t}, J=7.0 \mathrm{~Hz}, 3 \mathrm{H}) ;{ }^{13} \mathrm{C}$ NMR $\left(100 \mathrm{MHz}, \mathrm{CDCl}_{3}\right): \delta 200.27,176.31,158.45,147.62,146.36$, 136.94, 134.10, 129.57, 121.67, 113.62, 109.54, 107.74, 103.29, 101.11, 64.48, 57.76, 55.31, 34.37, 27.29, 14.24; HRMS (EI, [M] $]^{+}$) for $\mathrm{C}_{22} \mathrm{H}_{22} \mathrm{O}_{5}$ calcd. 366.1462, found: 366.1467. 
4-(Dimethylamino)-4'-ethoxy-4"'-(trifluoromethyl)-5',6'-dihydro-2'H-[1,1':1',1'-terphenyl]-2'one $(3 x)$

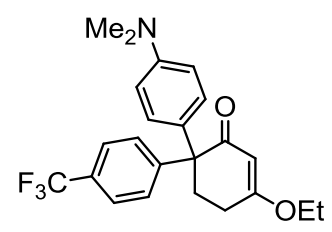

The reaction was conducted with $0.5 \mathrm{mmol}$ of $1 \mathrm{a}$ following the general procedure $\mathrm{C}$ (4-bromo-N,Ndimethylaniline then 4-bromobenzotrifluoride). The crude product was purified by flash column chromatography (hexanes/EtOAc $=8 / 1$ to $3 / 1)$ to afford $3 \mathbf{x}(110 \mathrm{mg}, 55 \%)$ as yellow solid (m.p. 59$60{ }^{\circ} \mathrm{C}$ ). $\mathrm{R}_{f}: 0.75$ (hexanes/ EtOAc = 1/1). IR (cast): 2941, 1654, 1610, 1521, 1326, $1185 \mathrm{~cm}^{-1} ;{ }^{1} \mathbf{H}$ NMR (400 MHz, $\left.\mathrm{CDCl}_{3}\right): \delta 7.50(\mathrm{~d}, J=8.1 \mathrm{~Hz}, 2 \mathrm{H}), 7.20(\mathrm{~d}, J=8.2 \mathrm{~Hz}, 2 \mathrm{H}), 7.07(\mathrm{~d}, J=8.8 \mathrm{~Hz}$, 2H), $6.70(\mathrm{~d}, J=8.8 \mathrm{~Hz}, 2 \mathrm{H}), 5.54(\mathrm{~s}, 1 \mathrm{H}), 3.95-3.81(\mathrm{~m}, 2 \mathrm{H}), 2.96(\mathrm{~s}, 6 \mathrm{H}), 2.67(\mathrm{t}, J=6.1 \mathrm{~Hz}, 2 \mathrm{H})$, 2.51-2.29 (m, 2H), $1.33(\mathrm{t}, J=7.0 \mathrm{~Hz}, 3 \mathrm{H}) ;{ }^{13} \mathbf{C}$ NMR (100 MHz, $\left.\mathrm{CDCl}_{3}\right): \delta$ 199.81, 176.38, 149.60, 148.83, 129.12, $128.68(\mathrm{q}, J=37.0 \mathrm{~Hz}), 127.25,124.83$ (q, $J=4.0 \mathrm{~Hz}), 124.38(\mathrm{q}, J=270.0 \mathrm{~Hz})$, 112.32, 103.28, 64.52, 57.91, 40.50, 34.03, 27.25, 14.25; ${ }^{19} \mathbf{F}$ NMR (375 $\left.\mathrm{MHz}, \mathrm{CDCl}_{3}\right):-63.82$; HRMS (EI, [M] $]^{+}$) for $\mathrm{C}_{23} \mathrm{H}_{24} \mathrm{~F}_{3} \mathrm{NO}_{2}$ calcd. 403.1754, found: 403.1750 .

\section{4'-Ethoxy-3,4-dimethoxy-5',6'-dihydro-2' $H-\left[1,1^{\prime}: 1^{\prime}, 1^{\prime \prime}: 4^{\prime \prime}, 1^{\prime \prime \prime}\right.$-quaterphenyl $]-2$ '-one (3y)}

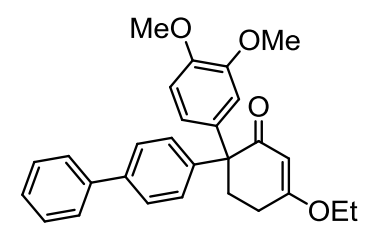

The reaction was conducted with $0.5 \mathrm{mmol}$ of $\mathbf{1 a}$ following the general procedure $\mathrm{C}$ (4-bromoveratrole then 4-bromobiphenyl). The crude product was purified by flash column chromatography (hexanes/EtOAc $=10 / 1$ to 3/1) to afford $\mathbf{3 y}\left(105 \mathrm{mg}, 49 \%\right.$ ) as light brown solid (m.p. 64-65 $\left.{ }^{\circ} \mathrm{C}\right)$. $\mathrm{R}_{f}$ : 0.4 (hexanes/ EtOAc = 3/1). IR (cast): 2953, 2935, 1652, 1608, $1516 \mathrm{~cm}^{-1}$; ${ }^{1} \mathbf{H}$ NMR (400 MHz, $\left.\mathrm{CDCl}_{3}\right): \delta 7.59(\mathrm{~d}, J=7.4 \mathrm{~Hz}, 2 \mathrm{H}), 7.53(\mathrm{~d}, J=7.6 \mathrm{~Hz}, 2 \mathrm{H}), 7.42(\mathrm{t}, J=7.3 \mathrm{~Hz}, 2 \mathrm{H}), 7.32(\mathrm{t}, J=6.9$ $\mathrm{Hz}, 1 \mathrm{H}), 7.19$ (d, $J=7.9 \mathrm{~Hz}, 2 \mathrm{H}), 6.84(\mathrm{~d}, J=8.0 \mathrm{~Hz}, 1 \mathrm{H}), 6.78(\mathrm{~d}, J=7.0 \mathrm{~Hz}, 2 \mathrm{H}), 5.58(\mathrm{~s}, 1 \mathrm{H}), 3.90-$ $3.88(\mathrm{~m}, 5 \mathrm{H}), 3.79(\mathrm{~s}, 3 \mathrm{H}), 2.79-2.66(\mathrm{~m}, 2 \mathrm{H}), 2.44(\mathrm{dd}, J=10.2,4.9 \mathrm{~Hz}, 2 \mathrm{H}), 1.34(\mathrm{t}, J=6.9 \mathrm{~Hz}, 3 \mathrm{H})$; ${ }^{13}$ C NMR (100 MHz, $\left.\mathrm{CDCl}_{3}\right): \delta 200.25,176.42,148.72,148.06,142.36,140.74,139.53,133.83$, $128.94,128.77,127.26,127.07,126.77,120.72$, 112.28, 110.65, 103.35, 64.47, 58.12, 55.94, 55.86, 34.29, 27.29, 14.20; HRMS (EI, [M] $]^{+}$) for $\mathrm{C}_{28} \mathrm{H}_{28} \mathrm{O}_{4}$ calcd. 428.1982, found: 428.1983. 
4'-Ethoxy-4-methoxy-3-methyl-5',6'-dihydro-2' $H$-[1,1':1',1'-terphenyl]-2'-one (3z)

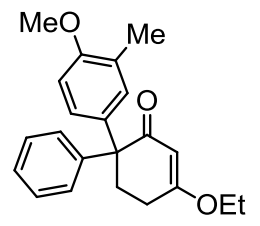

The reaction was conducted with $0.5 \mathrm{mmol}$ of $1 \mathrm{a}$ following the general procedure $\mathrm{C}$ (4-bromo-2methylanisole then bromobenzene). The crude product was purified by flash column chromatography (hexanes/EtOAc $=10 / 1$ to $3 / 1)$ to afford $\mathbf{3 z}\left(97 \mathrm{mg}, 58 \%\right.$ ) as yellow oil. $\mathrm{R}_{f}: 0.2$ (hexanes/ EtOAc $=$ 3/1). IR (film): 2980, 2941, 1654, 1609, $1504 \mathrm{~cm}^{-1}$; ${ }^{1} \mathbf{H}$ NMR (400 MHz, $\left.\mathrm{CDCl}_{3}\right): \delta$ 7.32-7.22 (m, 4H), $7.14(\mathrm{~d}, J=8.0 \mathrm{~Hz}, 2 \mathrm{H}), 6.99-6.91(\mathrm{~m}, 2 \mathrm{H}), 6.76(\mathrm{~d}, J=8.5 \mathrm{~Hz}, 1 \mathrm{H}), 5.55(\mathrm{~s}, 1 \mathrm{H}), 3.88$ (q, $J=7.0 \mathrm{~Hz}$, 3H), $3.82(\mathrm{~s}, 3 \mathrm{H}), 3.77(\mathrm{~s}, 1 \mathrm{H}), 2.70(\mathrm{t}, J=5.8 \mathrm{~Hz}, 2 \mathrm{H}), 2.38(\mathrm{q}, J=6.2 \mathrm{~Hz}, 2 \mathrm{H}), 2.19(\mathrm{~s}, 4 \mathrm{H}), 1.33(\mathrm{t}$, $J=7.0 \mathrm{~Hz}, 3 \mathrm{H}) ;{ }^{13} \mathbf{C} \mathbf{N M R}\left(100 \mathrm{MHz}, \mathrm{CDCl}_{3}\right): \delta 200.49,176.26,156.63,143.12,133.31,130.75$, $128.58,128.08,126.98,126.72,126.20,109.43,103.39,64.39,58.05,55.30,34.22,27.29,16.57$, 14.21; HRMS (EI, [M] $]^{+}$) for $\mathrm{C}_{22} \mathrm{H}_{24} \mathrm{O}_{3}$ calcd. 336.1720, found: 336.1716 .

\section{One-Pot Sequential Triaylation of 1c.}

$(1 ' S, 5 ' R)-5$ '-(3,4-Dimethoxyphenyl)-4'-ethoxy-3,4-dimethoxy-3',4' '-dimethyl-5',6' -dihydro-2' H[1,1':1',1'-terphenyl]-2'-one (4h) and (1'S,5'S)-5'-(3,4-Dimethoxyphenyl)-4'-ethoxy-3,4dimethoxy-3',4'"-dimethyl-5',6'-dihydro-2'H-[1,1':1',1' '-terphenyl]-2'-one (4h')
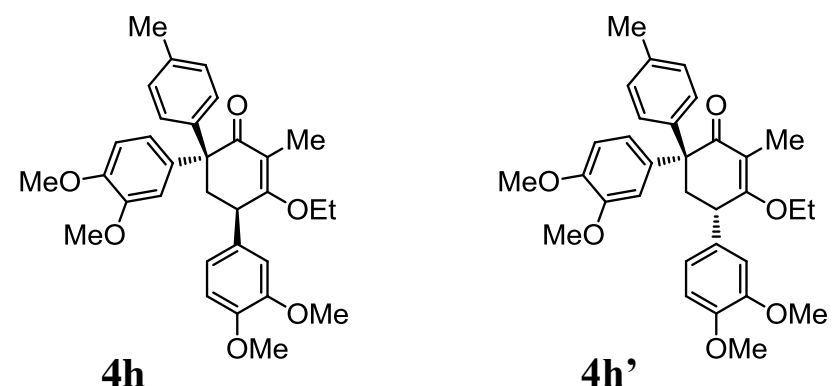

A round-bottom flask containing a vinylogous ester $(0.5 \mathrm{mmol})$, an aryl bromide (1.1 equiv), $\operatorname{Pd}(\mathrm{OAc})_{2}(10 \mathrm{~mol} \%, 11 \mathrm{mg})$ and $\mathrm{PAd}_{3}(12 \mathrm{~mol} \%, 26 \mathrm{mg})$ was vacuumed for 2 minutes. The whole system was backfilled with $\mathrm{N}_{2}$, and then $5 \mathrm{~mL}$ of toluene was added to the flask. To the stirring mixture was added a solution of LiHMDS (1 M, 7.0 equiv, $5.6 \mathrm{~mL})$ over 5 minutes at room temperature. The resulting mixture was then stirred at room temperature for 15 minutes. After completion of the first arylation, another aryl bromide (6.0 equiv) was added and the temperature was raised to $70{ }^{\circ} \mathrm{C}$ 
(temperature of the aluminum heating block). After stirring for 15 minutes, a saturated aqueous solution of $\mathrm{NH}_{4} \mathrm{Cl}$ was added to quench the reaction at room temperature. The reaction mixture was filtered through celite. The filtrate was diluted with ethyl acetate and extracted with water. The organic layers were dried over anhydrous $\mathrm{MgSO}_{4}$, filtered and concentrated under reduced pressure. The crude residue thus obtained was purified by flash column chromatography (hexanes/EtOAc $=10 / 1$ to $3 / 1$ ) to afford $4 \mathbf{h}(81 \mathrm{mg}, 31 \%)$ as yellow solid (m.p. $\left.72-73^{\circ} \mathrm{C}\right)$ and $\mathbf{4 h} \mathbf{h}^{\prime}(37 \mathrm{mg}, 14 \%)$ as yellow oil.

4h: $\mathrm{R}_{f}: 0.25$ (hexanes/ EtOAc = 3/1). IR (film): 2931, 2834, 1652, 1614, $1509 \mathrm{~cm}^{-1} ;{ }^{1} \mathbf{H}$ NMR (400 $\left.\mathrm{MHz}, \mathrm{CDCl}_{3}\right): \delta 6.98(\mathrm{~d}, J=8.2 \mathrm{~Hz}, 2 \mathrm{H}), 6.90-6.76(\mathrm{~m}, 5 \mathrm{H}), 6.72-6.70(\mathrm{~m}, 2 \mathrm{H}), 6.63(\mathrm{~d}, J=1.9 \mathrm{~Hz}$, $1 \mathrm{H}), 3.91(\mathrm{~s}, 3 \mathrm{H}), 3.87(\mathrm{~s}, 3 \mathrm{H}), 3.86(\mathrm{~s}, 3 \mathrm{H}), 3.80(\mathrm{~s}, 3 \mathrm{H}), 3.65(\mathrm{br} \mathrm{s}, 1 \mathrm{H}), 3.55-3.47(\mathrm{~m}, 2 \mathrm{H}), 2.85(\mathrm{dd}$, $J=13.9,5.0 \mathrm{~Hz}, 1 \mathrm{H}), 2.71(\mathrm{dd}, J=13.9,10.2 \mathrm{~Hz}, 1 \mathrm{H}), 2.25(\mathrm{~s}, 3 \mathrm{H}), 1.93(\mathrm{~d}, J=1.6 \mathrm{~Hz}, 3 \mathrm{H}), 0.92(\mathrm{t}$, $J=7.0 \mathrm{~Hz}, 3 \mathrm{H}) ;{ }^{13} \mathrm{C}$ NMR $\left(100 \mathrm{MHz}, \mathrm{CDCl}_{3}\right): \delta 199.99,169.78,149.48,148.87,148.15,147.99$, $141.30,136.08,134.56,133.89,128.54,128.45,120.29,120.12,120.10,111.74,111.68,110.79,64.86$, $57.85,56.14,56.07,56.03,55.96,44.88,42.40,21.04,15.38,9.60$ (a sp ${ }^{2}$ carbon signal missing due to peak overlap); HRMS (EI, [M] $]^{+}$) for $\mathrm{C}_{32} \mathrm{H}_{36} \mathrm{O}_{6}$ calcd. 516.2506, found: 516.2509.

4h': $\mathrm{R}_{f}: 0.2$ (hexanes/ EtOAc = 3/1). IR (film): 2933, 2834, 1652, 1615, $1512 \mathrm{~cm}^{-1} ;{ }^{1} \mathbf{H}$ NMR (400 $\mathrm{MHz}_{\mathrm{CDCl}}$ ): $\delta 7.15(\mathrm{~d}, J=8.2 \mathrm{~Hz}, 2 \mathrm{H}), 7.06(\mathrm{~d}, J=8.2 \mathrm{~Hz}, 2 \mathrm{H}), 6.80(\mathrm{~d}, J=8.2 \mathrm{~Hz}, 1 \mathrm{H}), 6.73-6.60$ $(\mathrm{m}, 3 \mathrm{H}), 6.48(\mathrm{~d}, J=8.7 \mathrm{~Hz}, 2 \mathrm{H}), 3.87(\mathrm{~s}, 3 \mathrm{H}), 3.86(\mathrm{~s}, 3 \mathrm{H}), 3.79(\mathrm{~s}, 3 \mathrm{H}), 3.67$ (s, 3H), 3.60 (br s, 1H), 3.54-3.48 (m, 2H), $2.90(\mathrm{dd}, J=13.7,5.0 \mathrm{~Hz}, 1 \mathrm{H}), 2.70(\mathrm{dd}, J=13.7,9.8 \mathrm{~Hz}, 1 \mathrm{H}), 2.36(\mathrm{~s}, 3 \mathrm{H}), 1.95$ $(\mathrm{d}, J=1.2 \mathrm{~Hz}, 3 \mathrm{H}), 0.92(\mathrm{t}, J=7.0 \mathrm{~Hz}, 3 \mathrm{H}) ;{ }^{13} \mathbf{C} \mathbf{N M R}\left(100 \mathrm{MHz}, \mathrm{CDCl}_{3}\right): \delta 199.92,169.71,149.40$, $148.07,147.66,139.05,136.61,136.54,134.45,129.12,127.91,120.76,120.13,113.13,111.64$, $110.90,110.23,64.74,57.62,56.14,56.06,55.92,55.87,44.47,42.30,21.16,15.32,9.54$ (two sp ${ }^{2}$ carbon signal missing due to peak overlap); HRMS (EI, [M] $]^{+}$) for $\mathrm{C}_{32} \mathrm{H}_{36} \mathrm{O}_{6}$ calcd. 516.2506, found: 516.2508 .

\section{Synthesis of Spiro-Fluorenes (Scheme 7).}

\section{4-Ethoxyspiro[cyclohexane-1,10'-fluoreno[2,3-d:6,7-d']bis([1,3]dioxole)]-3-en-2-one (6)}

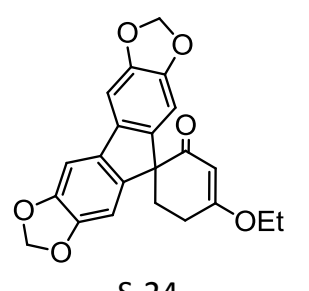


A round-bottom flask containing 3a $(0.5 \mathrm{mmol})$, DDQ (2,3-dichloro-5,6-dicyano-1,4benzoquinone; 1.0 equiv, $114 \mathrm{mg})$, iron(III) chloride (10.0 equiv, $810 \mathrm{mg}$ ) and $50 \mathrm{~mL}$ of dichloromethane was stirred at $0{ }^{\circ} \mathrm{C}$ for 15 minutes. After completion of the reaction as indicated by TLC, a saturated aqueous solution of $\mathrm{NaHCO}_{3}$ was added to quench the reaction at room temperature. The reaction mixture was filtered through celite. The filtrate was diluted with ethyl acetate and extracted with water. The organic layers were dried over anhydrous $\mathrm{MgSO}_{4}$, filtered and concentrated under reduced pressure. The crude residue thus obtained was purified by flash column chromatography (hexanes/EtOAc $=5 / 1$ to $1 / 1)$ to afford $6\left(126 \mathrm{mg}, 67 \%\right.$ ) as creamy-white solid (m.p. $\left.216-217^{\circ} \mathrm{C}\right) . \mathrm{R}_{f}$ : 0.5 (hexanes/ EtOAc = 1/1). IR (cast): 2902, 1652, 1602, 1472, $1249 \mathrm{~cm}^{-1} ;{ }^{1} \mathbf{H}$ NMR $(400 \mathrm{MHz}$, $\left.\mathrm{CDCl}_{3}\right): \delta 7.04(\mathrm{~s}, 2 \mathrm{H}), 6.81(\mathrm{~s}, 2 \mathrm{H}), 5.98(\mathrm{~d}, J=11.0 \mathrm{~Hz}, 4 \mathrm{H}), 5.65(\mathrm{~s}, 1 \mathrm{H}), 4.05(\mathrm{q}, J=7.0 \mathrm{~Hz}, 2 \mathrm{H})$, $2.76(\mathrm{t}, J=6.3 \mathrm{~Hz}, 2 \mathrm{H}), 2.22(\mathrm{t}, J=6.3 \mathrm{~Hz}, 2 \mathrm{H}), 1.46(\mathrm{t}, J=7.0 \mathrm{~Hz}, 3 \mathrm{H}) ;{ }^{13} \mathbf{C ~ N M R}\left(100 \mathrm{MHz}, \mathrm{CDCl}_{3}\right)$ : $\delta 197.91,176.89,148.06,146.84,140.95,135.09,105.07,103.97,101.45,100.55,64.83,60.19,32.69$, 27.23, 14.36; HRMS (EI, [M] $]^{+}$) for $\mathrm{C}_{22} \mathrm{H}_{18} \mathrm{O}_{6}$ calcd. 378.1103, found: 378.1105 .

\section{Spiro[cyclohexane-1,10'-fluoreno[2,3-d:6,7-d']bis([1,3]dioxole)]-2-en-4-one (7)}

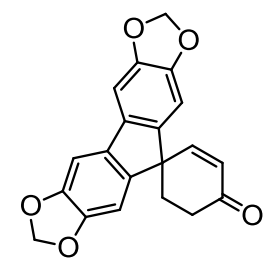

A round-bottom flask containing $6(0.5 \mathrm{mmol})$ was vacuumed for 30 minutes. The whole system was backfilled with $\mathrm{N}_{2}$, and then $8 \mathrm{~mL}$ of toluene and $2 \mathrm{~mL}$ of tetrahydrofuran were added to the flask. To the stirring mixture was added a solution of DIBAL ( $1 \mathrm{M}$ in toluene, 3.0 equiv, $1.5 \mathrm{~mL}$ ) over 5 minutes at $-20^{\circ} \mathrm{C}$. The resulting mixture was then stirred at $-20^{\circ} \mathrm{C}$ for 2 hours. After completion of the reaction as indicated by TLC, water was added to quench the reaction at $-20{ }^{\circ} \mathrm{C}$, and the mixture was allowed to warm to room temperature. A $1 \mathrm{M}$ aqueous solution of $\mathrm{HCl}(1.5$ equiv, $0.75 \mathrm{~mL})$ was slowly added to the reaction mixture at room temperature. After $1 \mathrm{~h}$, a saturated aqueous solution of $\mathrm{NaHCO}_{3}$ was added to neutralize the reaction at room temperature. The reaction mixture was filtered through celite. The filtrate was diluted with ethyl acetate and extracted with water. The organic layers were dried over anhydrous $\mathrm{MgSO}_{4}$, filtered and concentrated under reduced pressure. The crude residue thus obtained 
was purified by flash column chromatography (hexanes/EtOAc $=8 / 1$ to $1 / 1)$ to afford 7 (60.5 mg, 91 \%) as yellow solid (decomposed at $\left.290-291{ }^{\circ} \mathrm{C}\right) . \mathrm{R}_{f}: 0.3$ (hexanes/ EtOAc $\left.=1 / 1\right)$. IR (cast): 2920, 1674, 1614, 1472, $1438 \mathrm{~cm}^{-1} ;{ }^{1} \mathbf{H}$ NMR (400 MHz, $\left.\mathrm{CDCl}_{3}\right): \delta 7.06$ (s, 2H), 6.87 (s, 2H), 6.43 (d, $J=$ $10.0 \mathrm{~Hz}, 1 \mathrm{H}), 6.24(\mathrm{~d}, J=10.0 \mathrm{~Hz}, 1 \mathrm{H}), 6.01(\mathrm{~s}, 4 \mathrm{H}), 2.81(\mathrm{t}, J=6.9 \mathrm{~Hz}, 2 \mathrm{H}), 2.27(\mathrm{t}, J=6.9 \mathrm{~Hz}, 2 \mathrm{H})$; ${ }^{13}$ C NMR $\left(100 \mathrm{MHz}, \mathrm{CDCl}_{3}\right): \delta 198.99,153.07,148.43,147.20,142.18,134.47,129.95,105.30$, 101.65, 100.38, 51.42, 35.10, 34.04; HRMS (EI, [M] $]^{+}$) for $\mathrm{C}_{20} \mathrm{H}_{14} \mathrm{O}_{5}$ calcd. 334.0841, found: 334.0845.

\section{3,3-Di-p-Tolyldihydrofuran-2(3H)-one (8)}

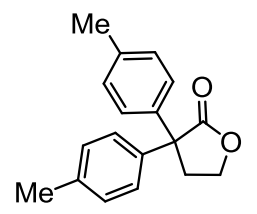

A round-bottom flask containing a gamma-butyrolactone $(0.5 \mathrm{mmol}), 4$-bromotoluene $(3.0$ equiv, $513 \mathrm{mg}), \operatorname{Pd}(\mathrm{OAc})_{2}(10 \mathrm{~mol} \%, 11 \mathrm{mg})$ and $\mathrm{PAd}_{3}(12 \mathrm{~mol} \%, 26 \mathrm{mg})$ was vacuumed for 2 minutes. The whole system was backfilled with $\mathrm{N}_{2}$, and then $5 \mathrm{~mL}$ of toluene was added to the flask. To the stirring mixture was added a solution of LiHMDS (1 M, 3.0 equiv, $2.4 \mathrm{~mL})$ over 3 minutes at room temperature. The resulting mixture was then stirred at $70{ }^{\circ} \mathrm{C}$ (temperature of the aluminum heating block) for 30 minutes. After completion of the reaction, a saturated aqueous solution of $\mathrm{NH}_{4} \mathrm{Cl}$ was added to quench the reaction at room temperature. The reaction mixture was filtered through celite. The filtrate was diluted with ethyl acetate and extracted with water. The organic layers were dried over anhydrous $\mathrm{MgSO}_{4}$, filtered and concentrated under reduced pressure. The crude residue thus obtained was purified by flash column chromatography (hexanes/EtOAc $=10 / 1$ to 3/1) to afford 8 (44 $\mathrm{mg}, 33$ \%) as yellow solid (m.p. 83-84 ${ }^{\circ} \mathrm{C}$ ). $\mathrm{R}_{f}$ : 0.375 (hexanes/ EtOAc = 3/1). IR (film): 2954, 2919, 1769, 1511, $1368 \mathrm{~cm}^{-1} ;{ }^{1} \mathbf{H}$ NMR (400 MHz, $\left.\mathrm{CDCl}_{3}\right): \delta 7.23(\mathrm{~d}, J=8.3 \mathrm{~Hz}, 4 \mathrm{H}), 7.14(\mathrm{~d}, J=8.3 \mathrm{~Hz}, 4 \mathrm{H})$, $4.28(\mathrm{t}, J=6.5 \mathrm{~Hz}, 2 \mathrm{H}), 2.95(\mathrm{t}, J=6.5 \mathrm{~Hz}, 2 \mathrm{H}), 2.33(\mathrm{~s}, 6 \mathrm{H}) ;{ }^{13} \mathbf{C} \mathbf{N M R}\left(100 \mathrm{MHz}, \mathrm{CDCl}_{3}\right): \delta 178.05$, 137.87, 137.28, 129.43, 127.54, 65.25, 55.92, 37.72, 21.09; HRMS (EI, [M] ${ }^{+}$) for $\mathrm{C}_{18} \mathrm{H}_{18} \mathrm{O}_{2}$ calcd. 266.1301, found: 266.1297. 


\section{ORTEP Diagram of $3 \mathrm{~g}$}

CCDC 1943074 contains the supplementary crystallographic data for this paper. These data can be obtained free of charge via www.ccdc.cam.ac.uk/data_request/cif, or by emailing data_request@ccdc.cam.ac.uk, or by contacting The Cambridge Crystallographic Data Centre, 12 Union Road, Cambridge CB2 1EZ, UK; fax: +44 1223336033.

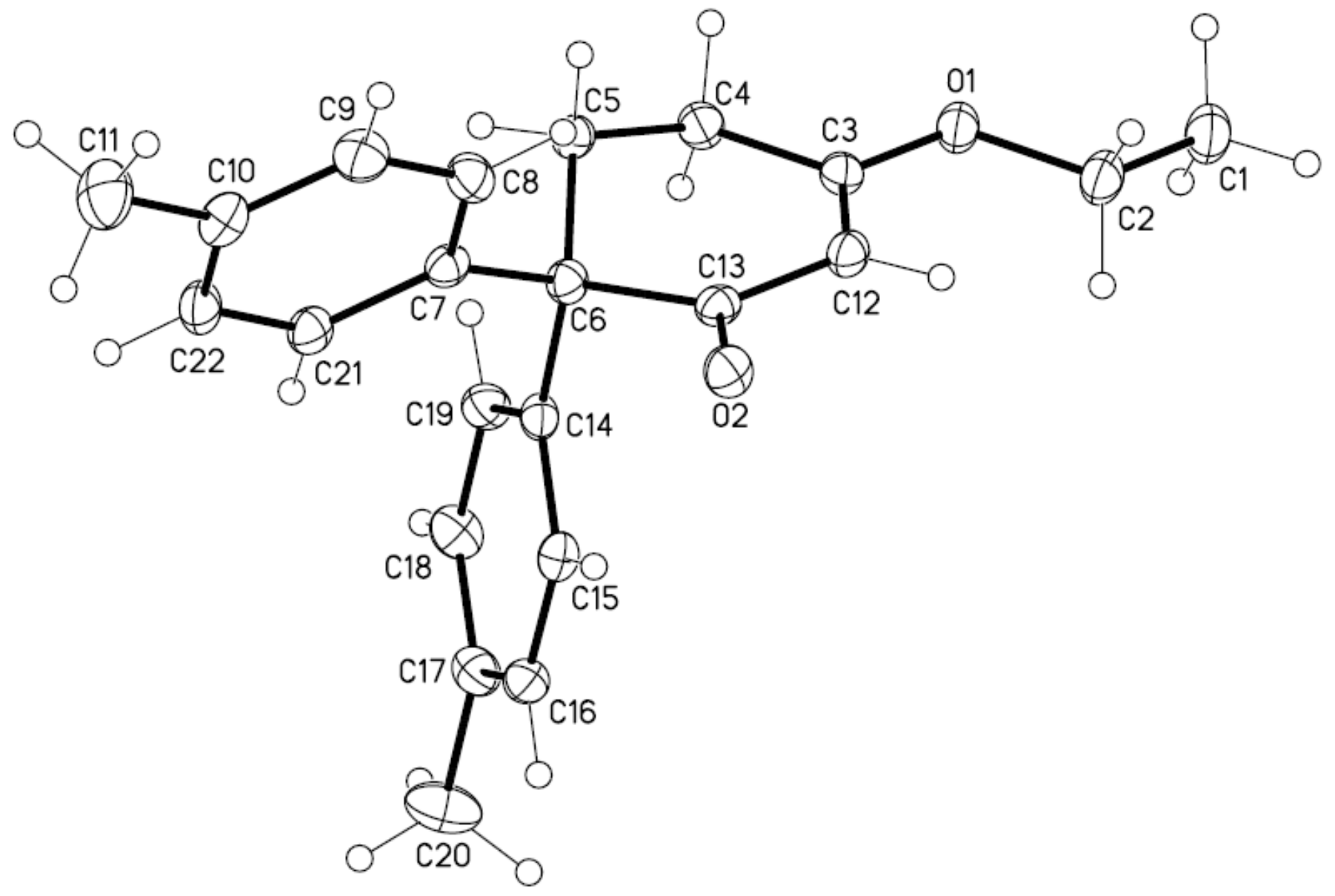




\section{ORTEP Diagram of $4 \mathrm{~g}$}

CCDC 1943073 contains the supplementary crystallographic data for this paper. These data can be obtained free of charge via www.ccdc.cam.ac.uk/data_request/cif, or by emailing data_request@ccdc.cam.ac.uk, or by contacting The Cambridge Crystallographic Data Centre, 12 Union Road, Cambridge CB2 1EZ, UK; fax: +44 1223336033.

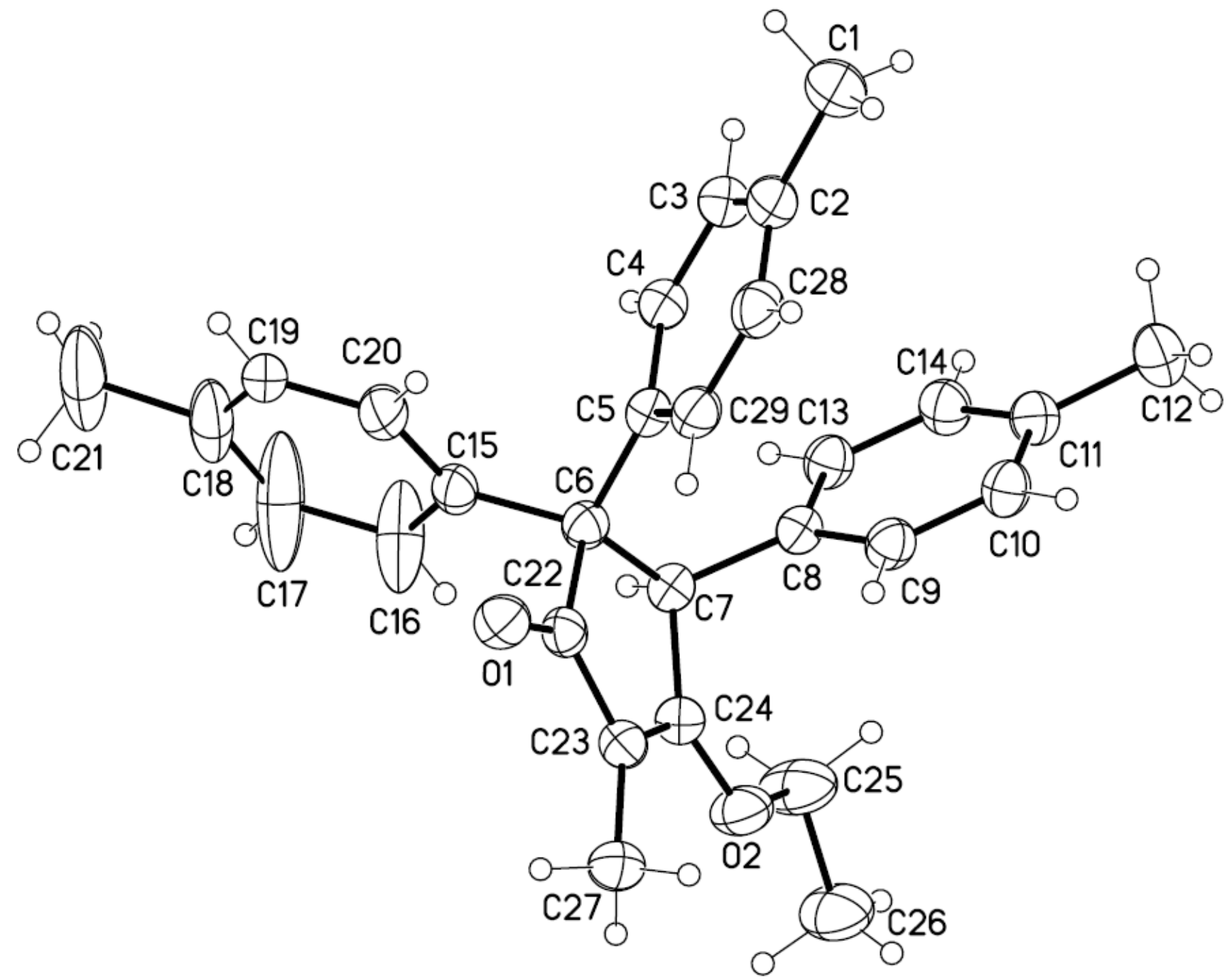




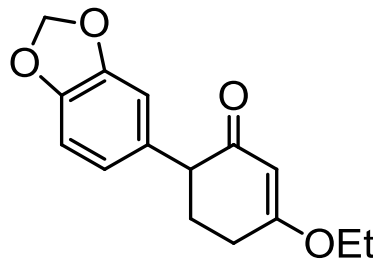


CARBON_01

${ }^{13} \mathrm{C}$ NMR spectrum of compound 2a

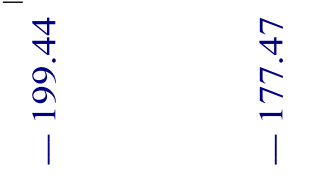

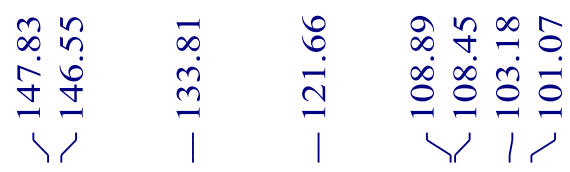

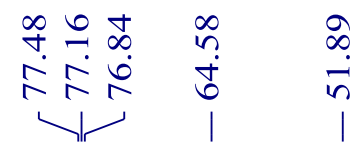

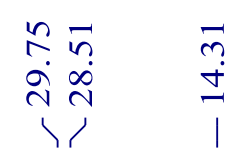

$-120$

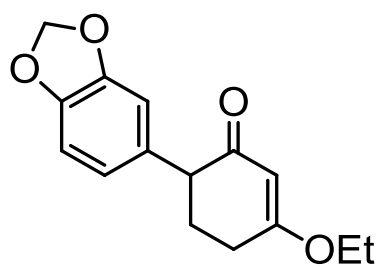

2a

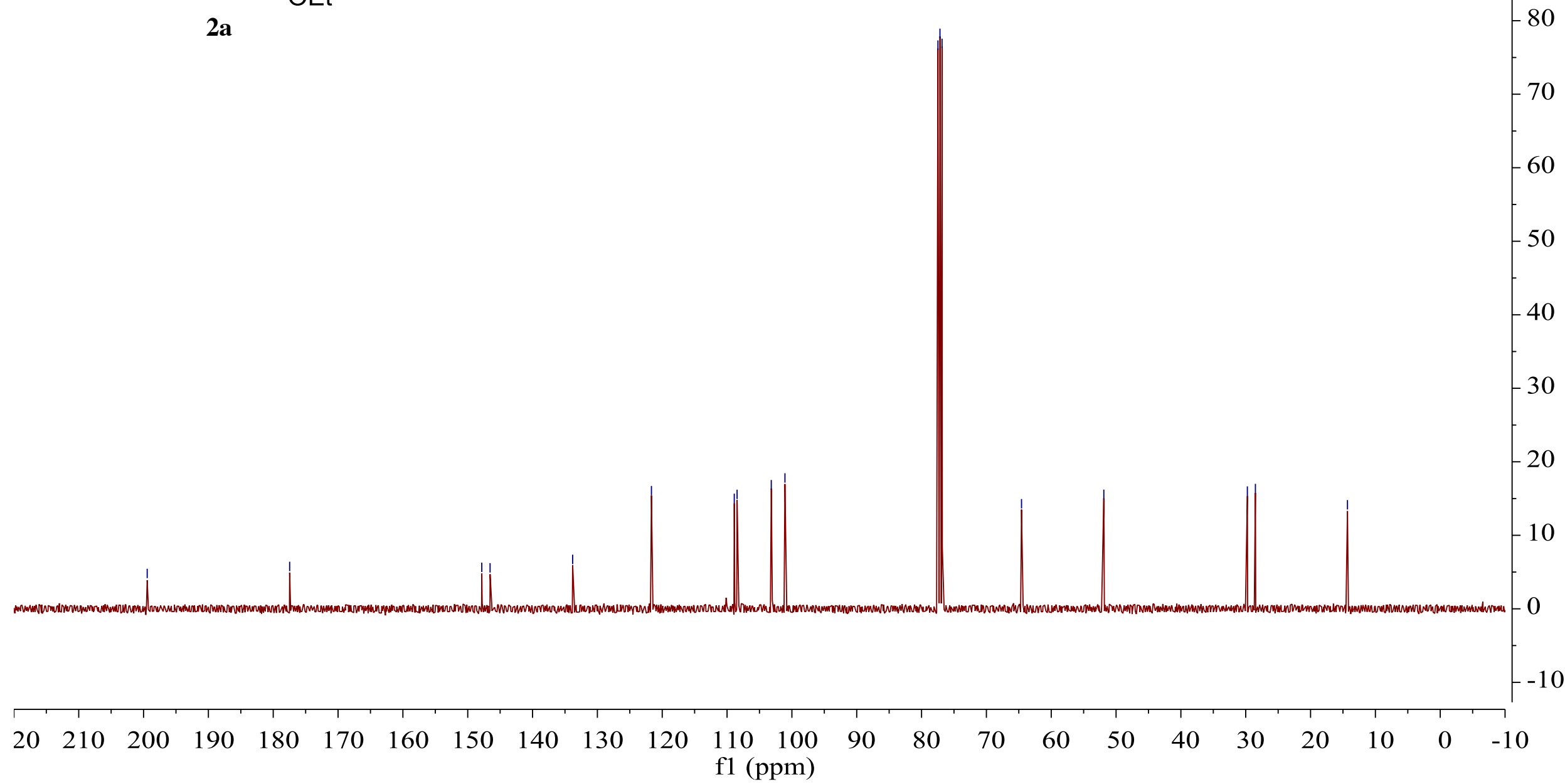




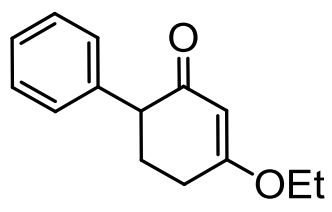

$2 b$

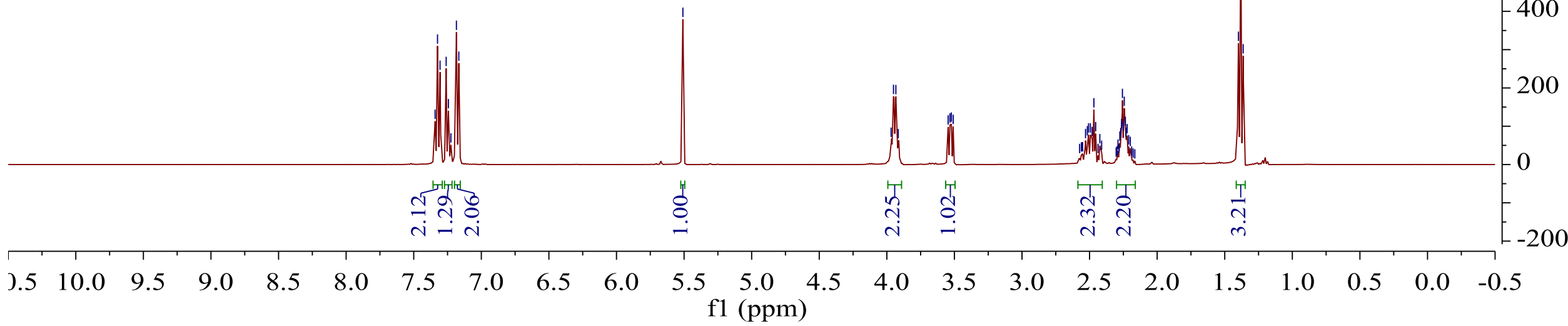


CARBON_01

${ }^{13} \mathrm{C}$ NMR spectrum of compound $2 \mathrm{~b}$

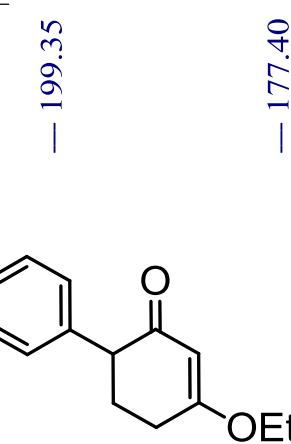

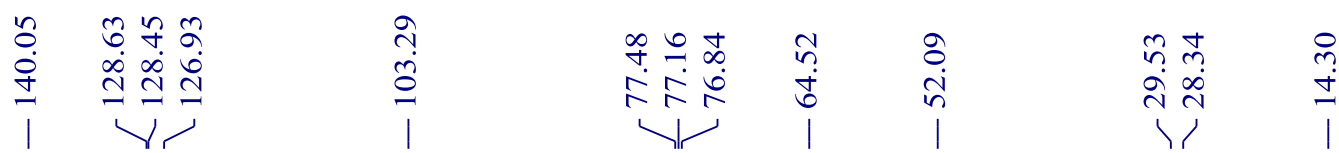

300

$2 \mathbf{b}$

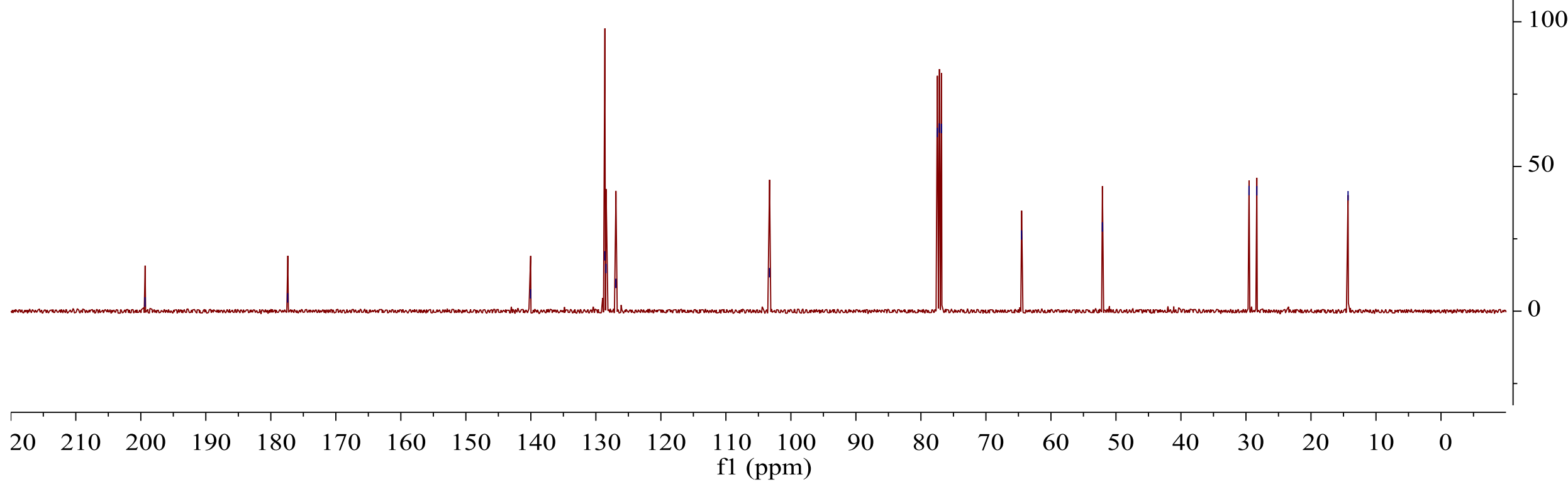


${ }^{1} \mathrm{H}$ NMR spectrum of compound $3 \mathrm{~b}$

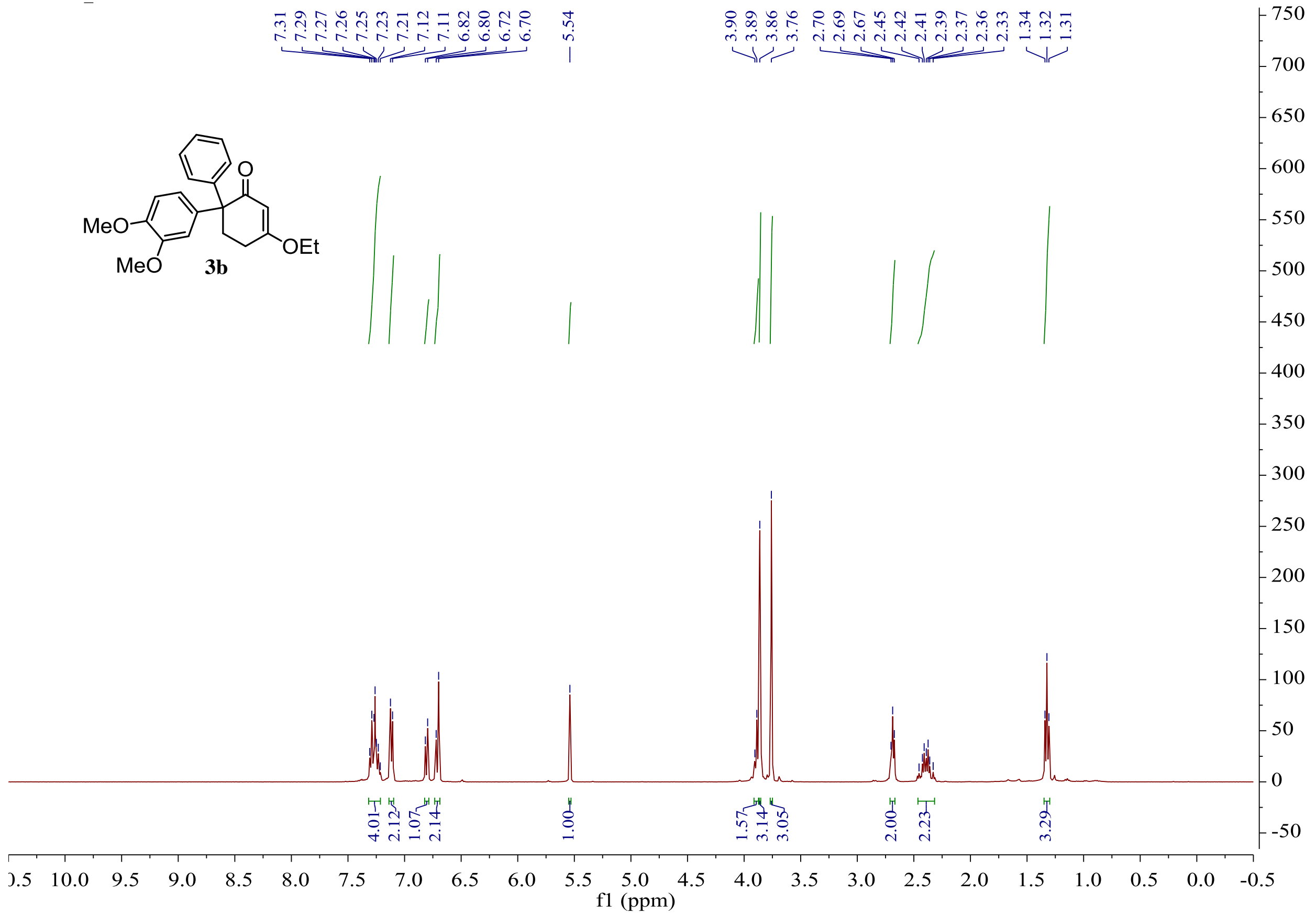


CARBON_01

${ }^{13} \mathrm{C}$ NMR spectrum of compound $3 b$

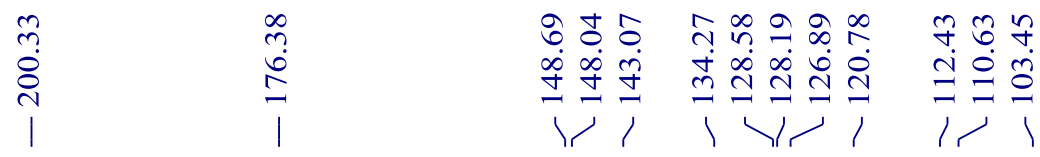

소용

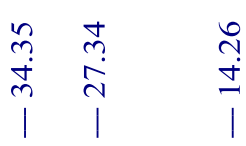
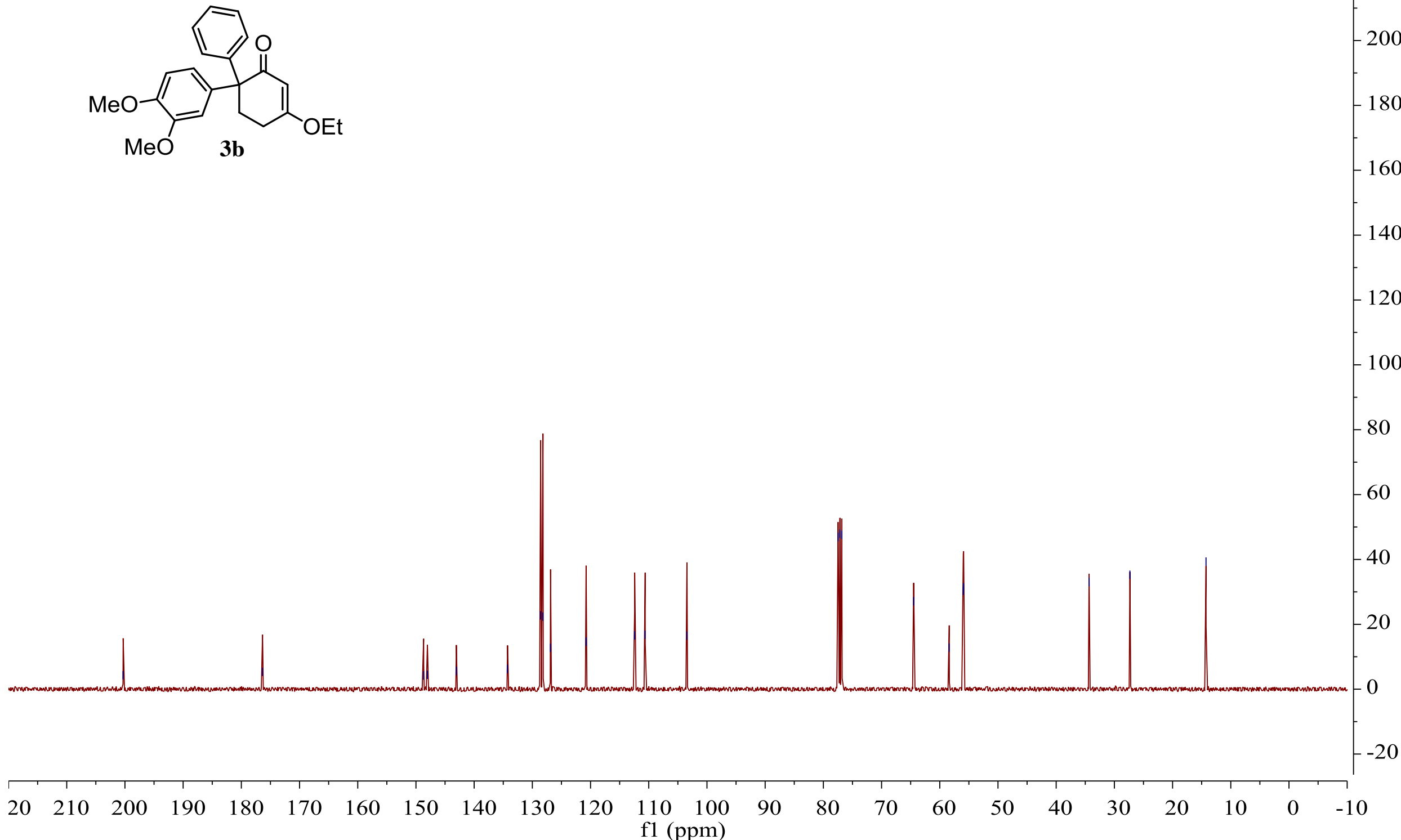


\section{${ }^{1} \mathrm{H}$ spectrum of compound $3 \mathbf{a}$}

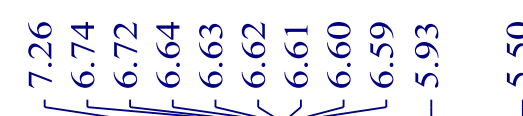

จे के क्ष

$m m m$

4

ํํㅇ :

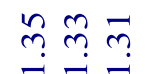

N n n n

$\rightarrow$

r

500

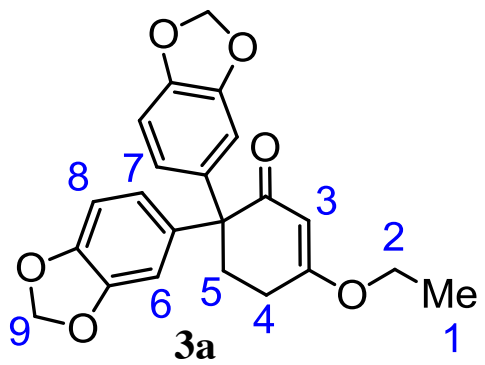

ㄷํำ

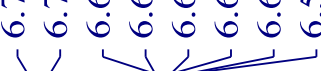

$-30099$
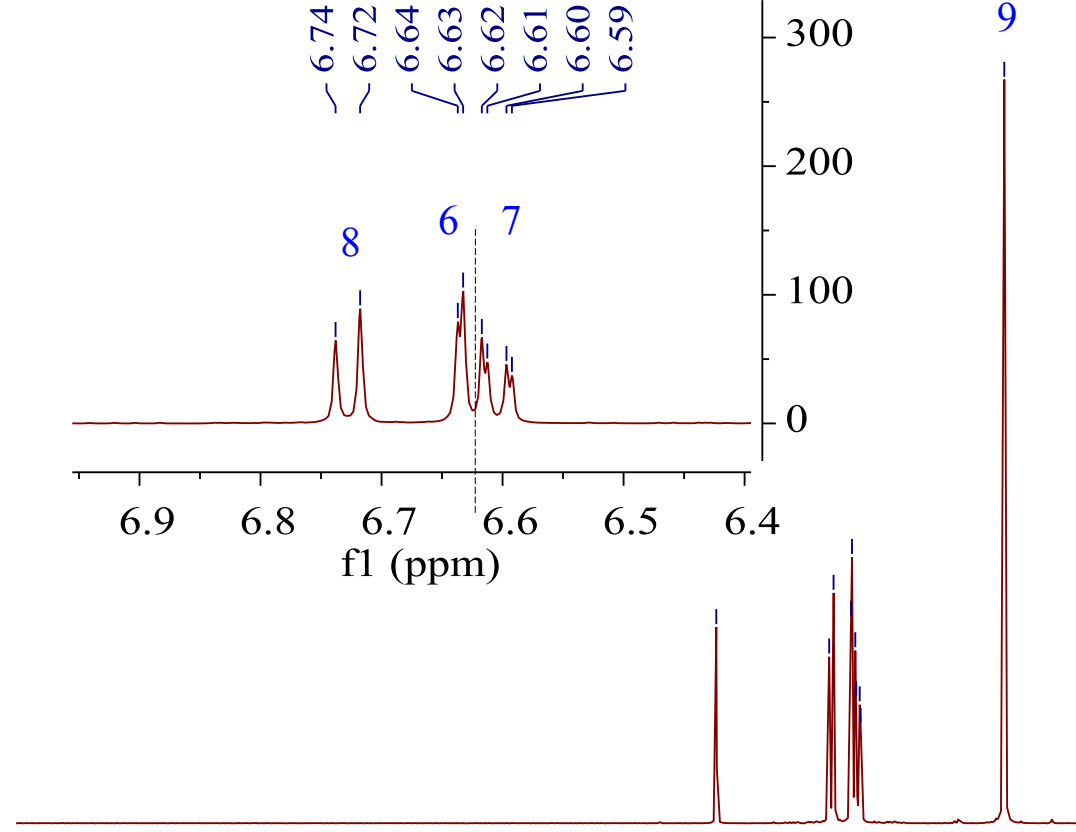

3

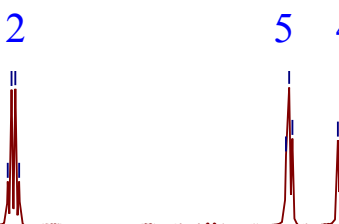

4

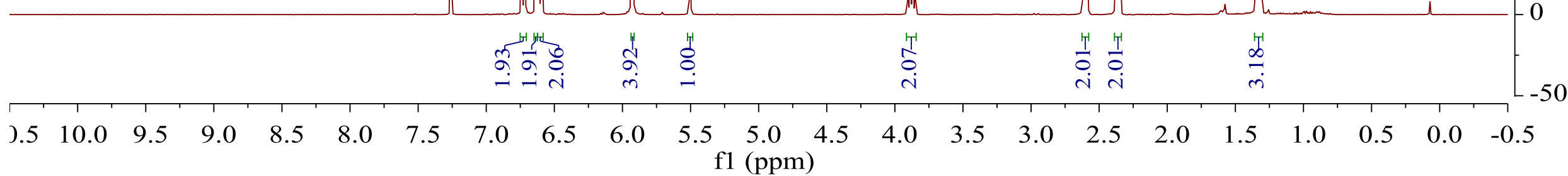


${ }^{13} \mathrm{C}$ NMR spectrum of compound $3 \mathrm{a}$

CARBON 01

ลे

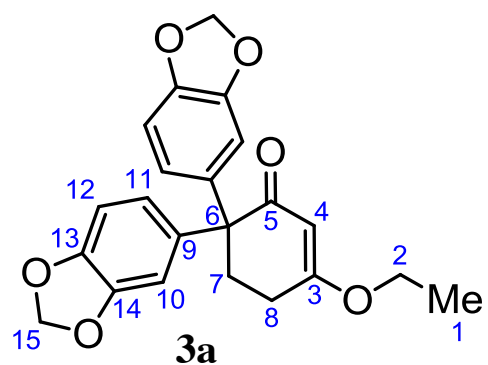

के

$$
\begin{aligned}
& \stackrel{+}{+} \\
& \stackrel{0}{1} \\
& 1
\end{aligned}
$$

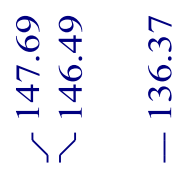

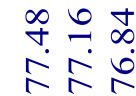

$\begin{array}{cc}n & m \\ n & \infty \\ \dot{b} & \infty \\ 1 & 1\end{array}$

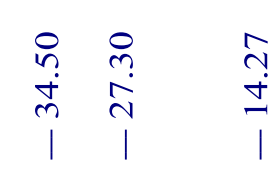

$\stackrel{ヘ}{ \pm}$

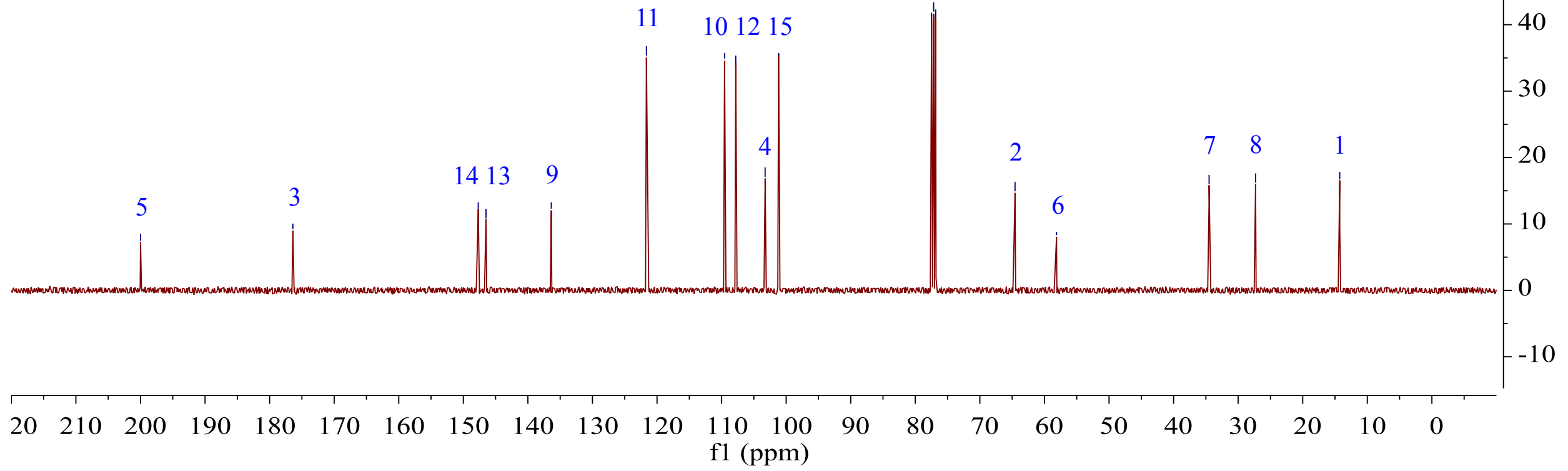


gCOSY_01

COSY spectrum of compound $3 a$

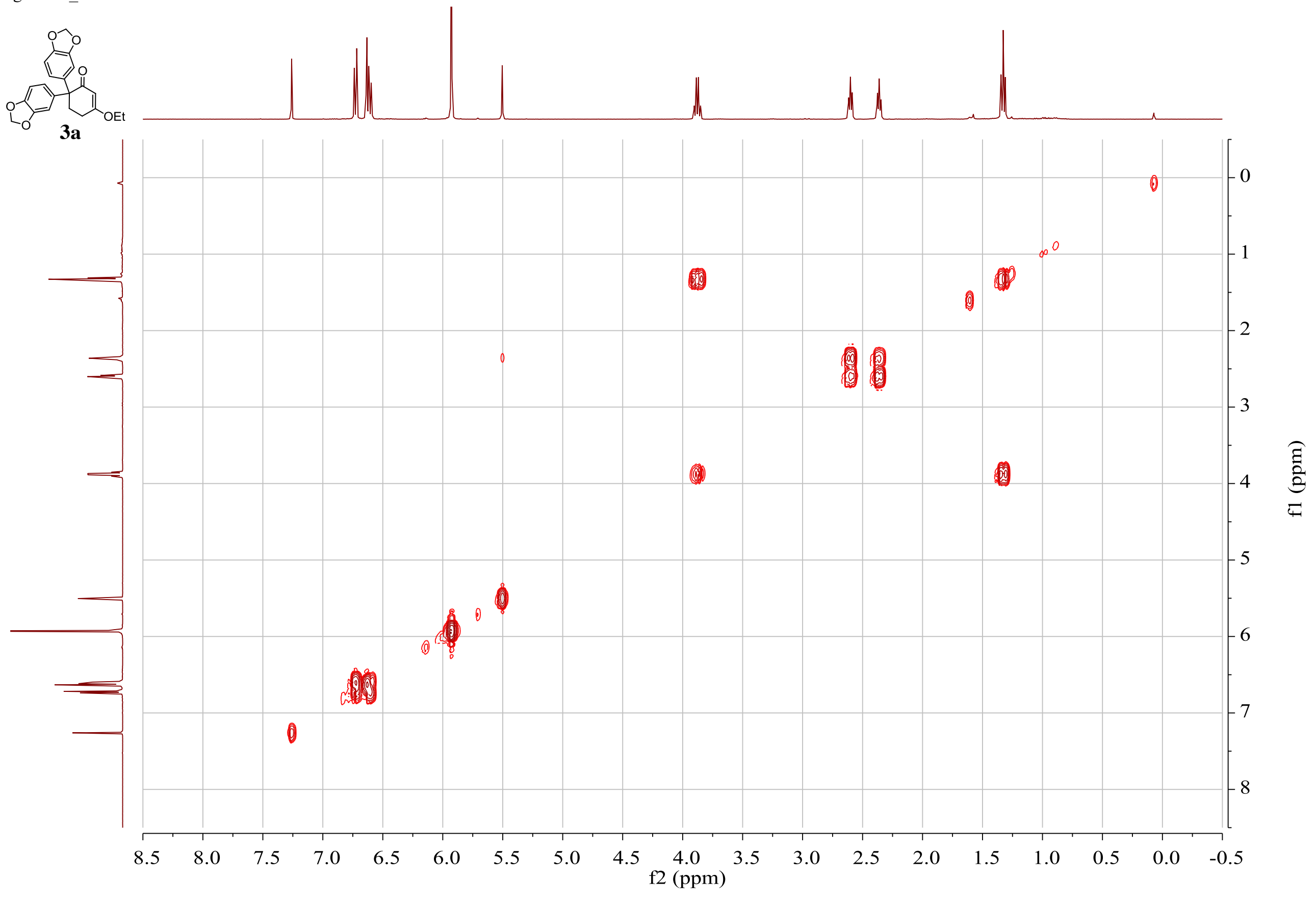


gHSQCAD_01

HSQC spectrum of compound 3a

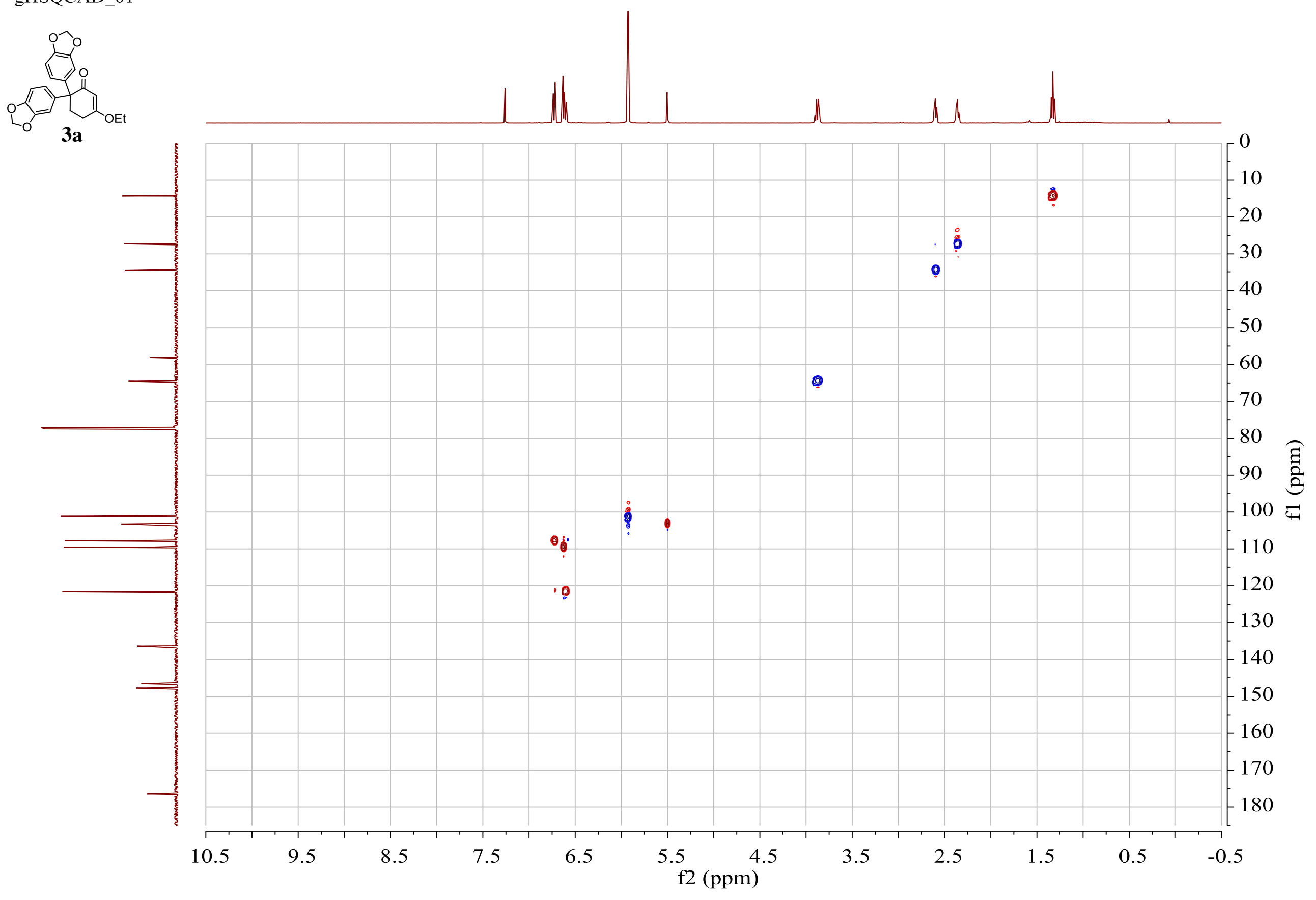




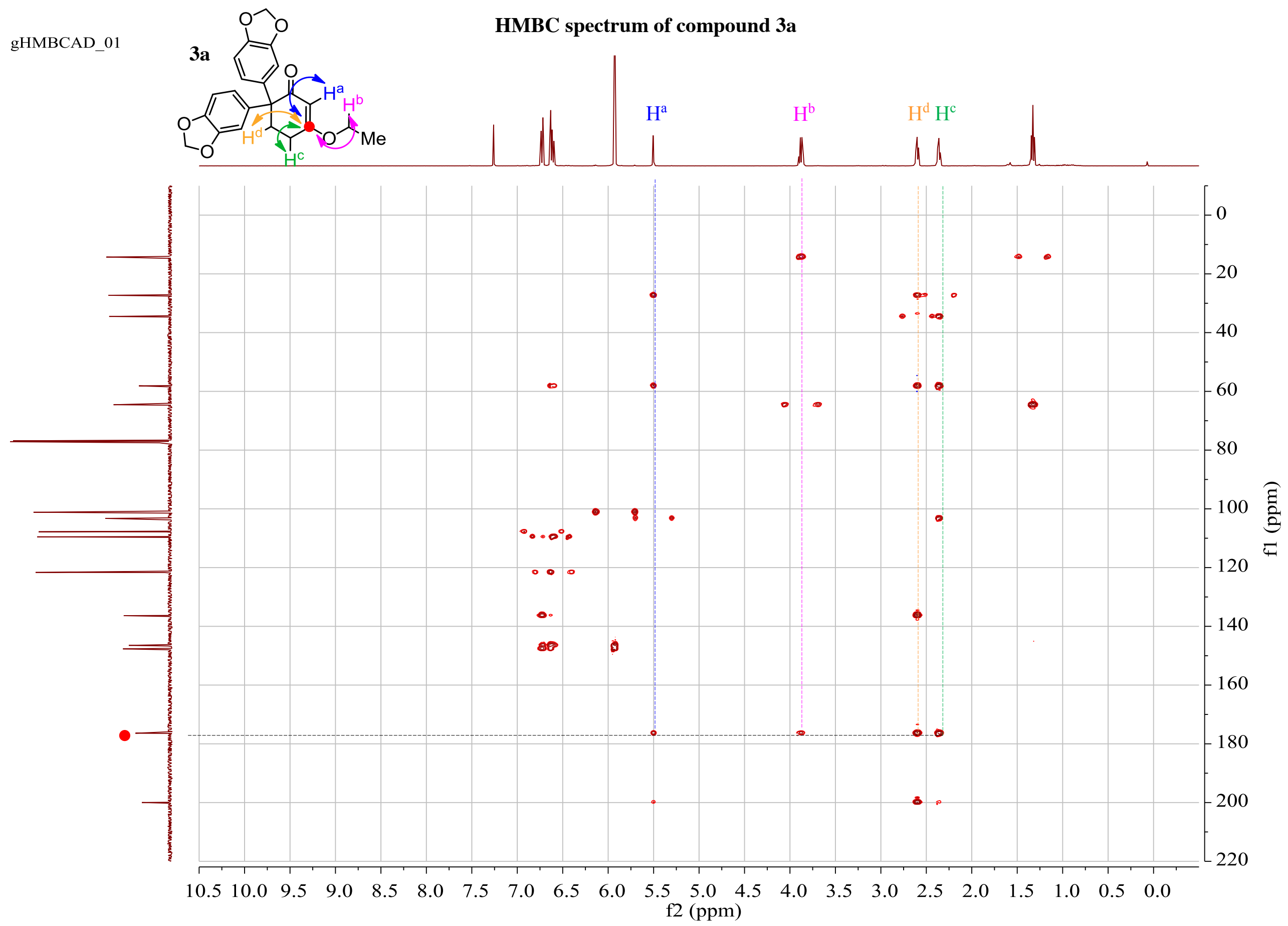


PROTON 01

${ }^{1}$ H NMR spectrum of compound $3 \mathrm{c}$

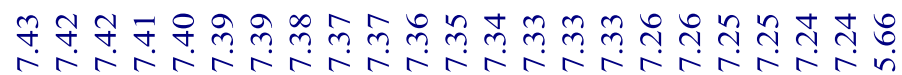

రㅇำ

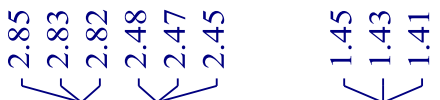

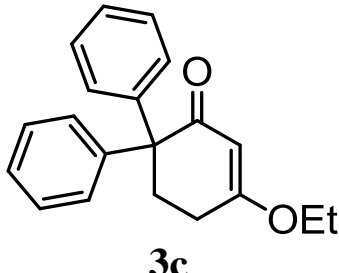

3c

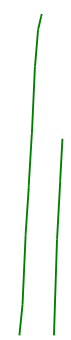

2000

1800

1600

1400
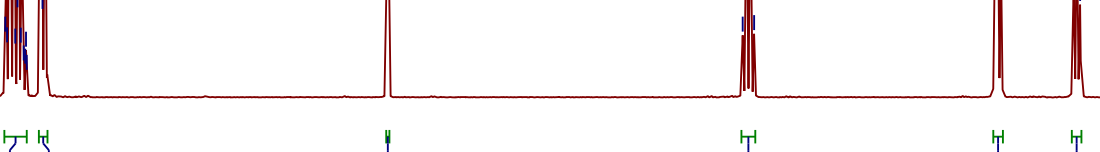

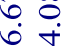

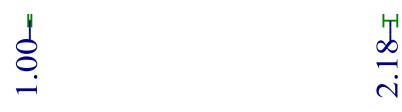

$\begin{array}{lll}0.5 & 10.0 \quad 9.5\end{array}$

8.5

8.0

7.5

$\begin{array}{ll}7.0 & 6.5\end{array}$

6.0

f1 $(\mathrm{ppm})$ 
CARBON_01

${ }^{13} \mathrm{C}$ NMR spectrum of compound $3 \mathrm{c}$

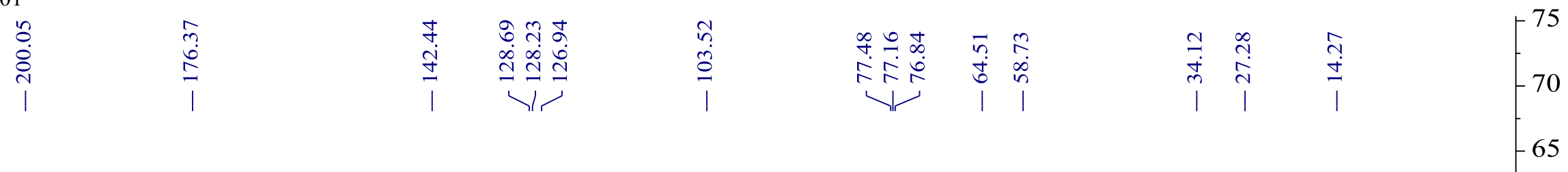

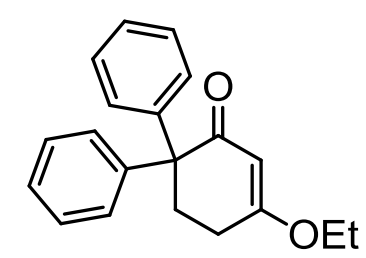

3c

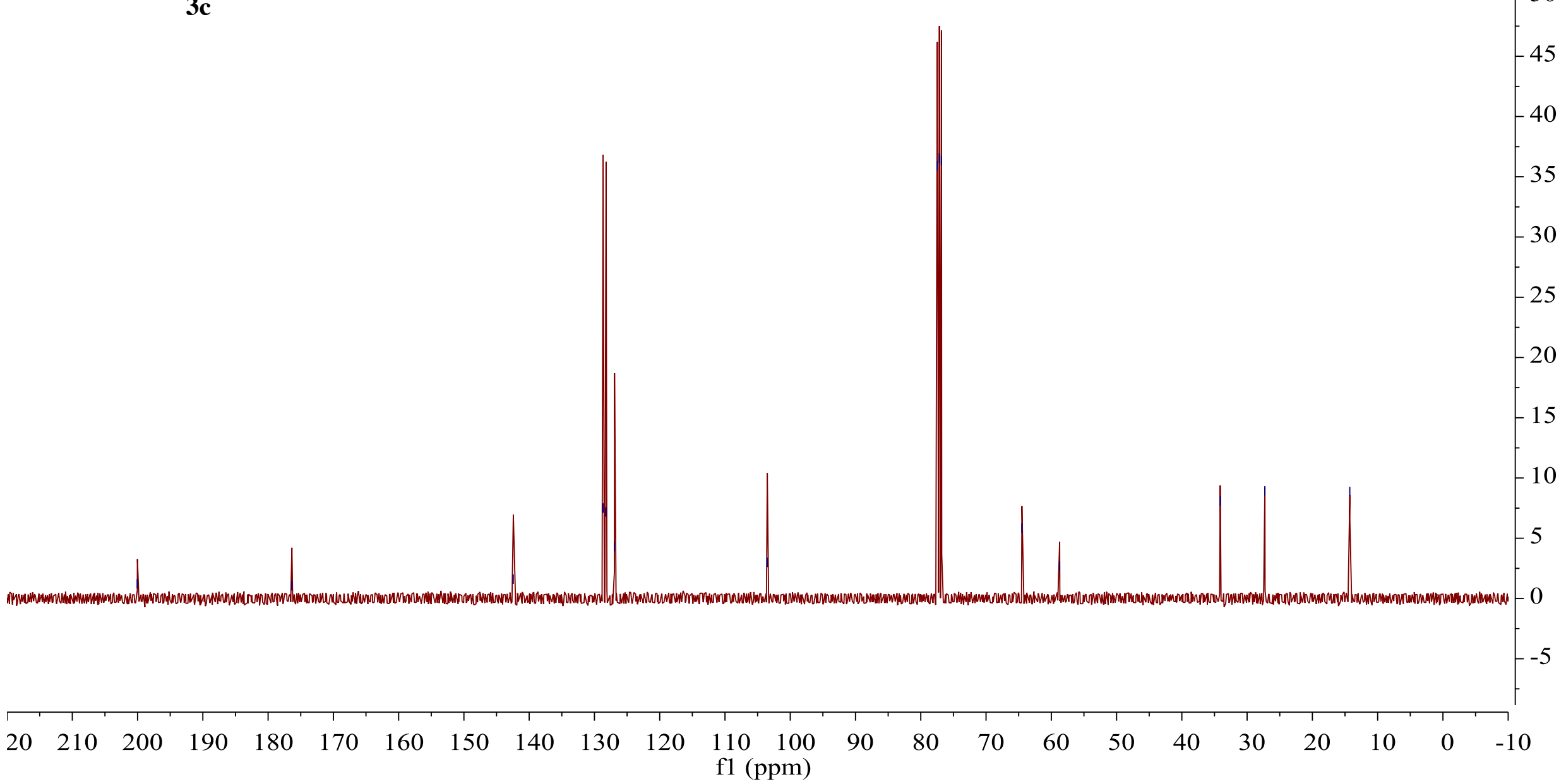


${ }^{1} \mathrm{H}$ NMR spectrum of compound $3 \mathrm{~d}$

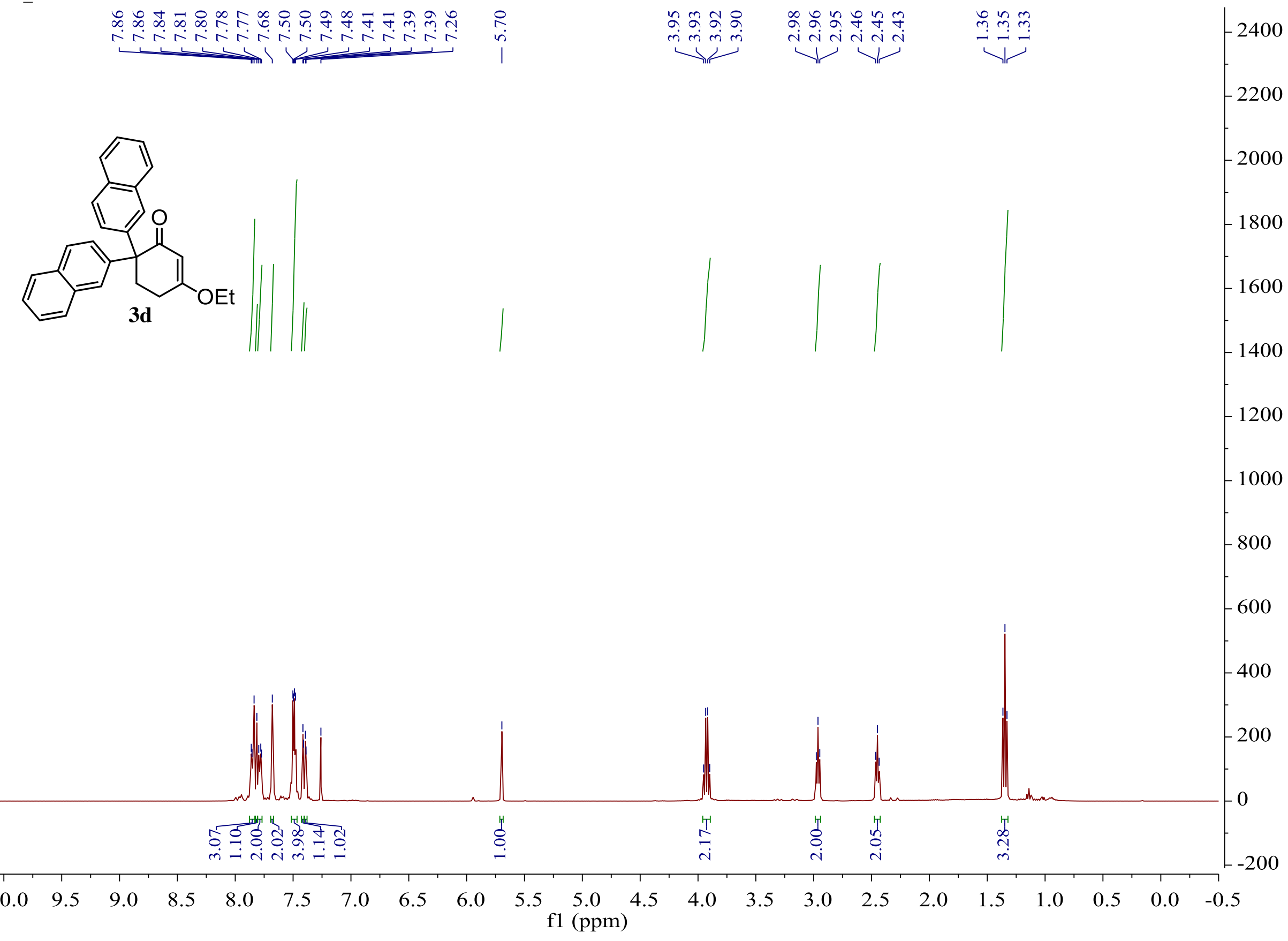


CARBON_01

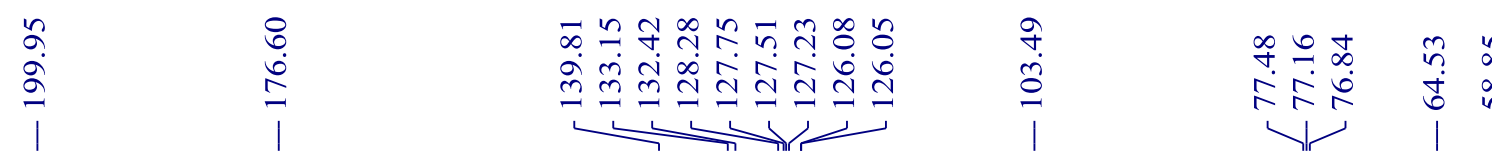

${ }^{13} \mathrm{C}$ NMR spectrum of compound $3 \mathrm{~d}$
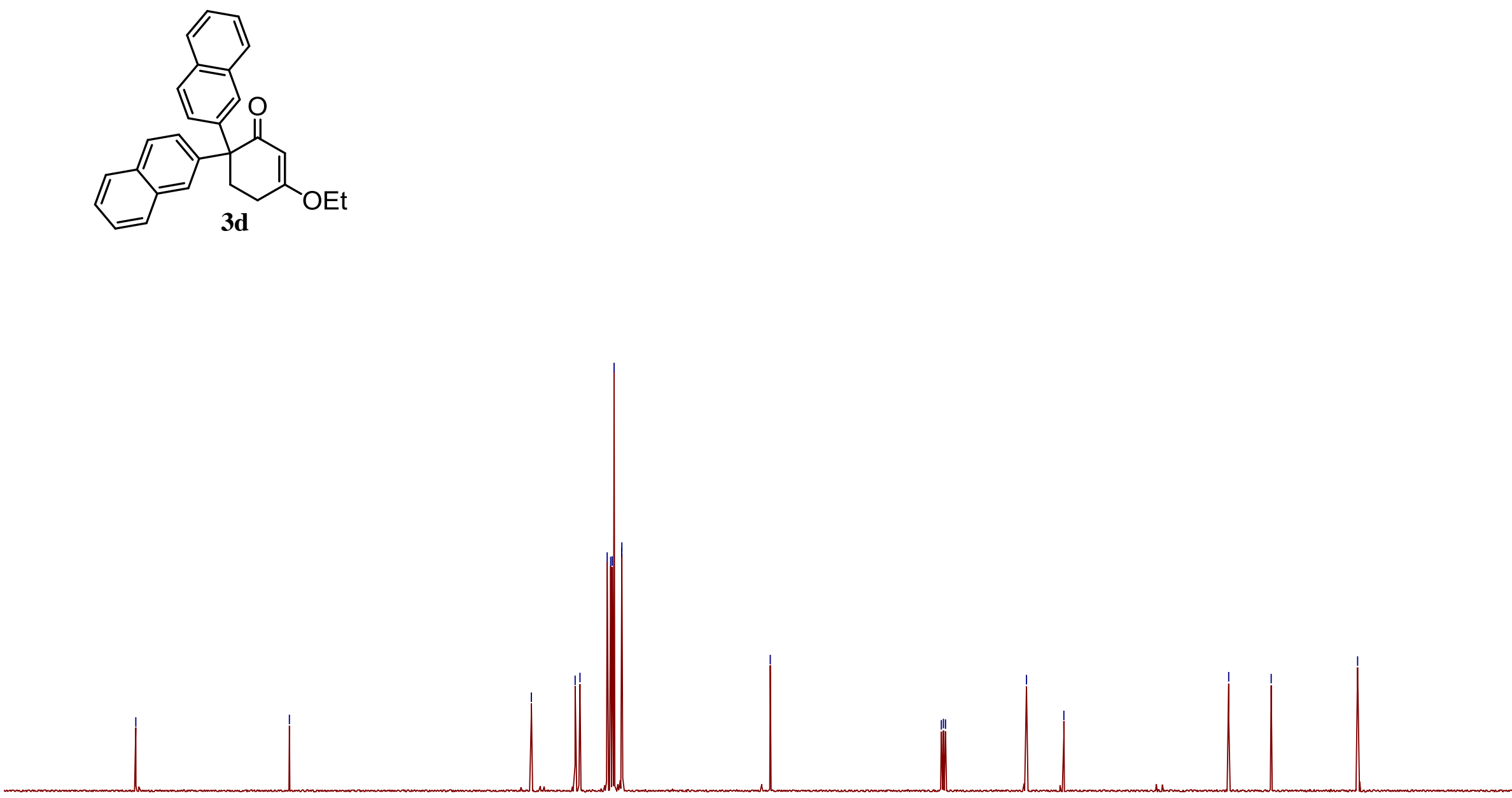


\section{${ }^{1} \mathrm{H}$ NMR spectrum of compound $3 \mathrm{e}$}

\begin{tabular}{|c|c|c|c|}
\hline 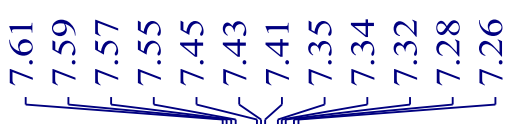 & $\begin{array}{c}\vec{b} \\
\dot{r}\end{array}$ & $\begin{array}{l}\dot{\sigma} \sigma \vec{\sigma} \infty \\
\dot{n} \dot{n} \dot{m}\end{array}$ & 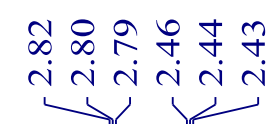 \\
\hline
\end{tabular}
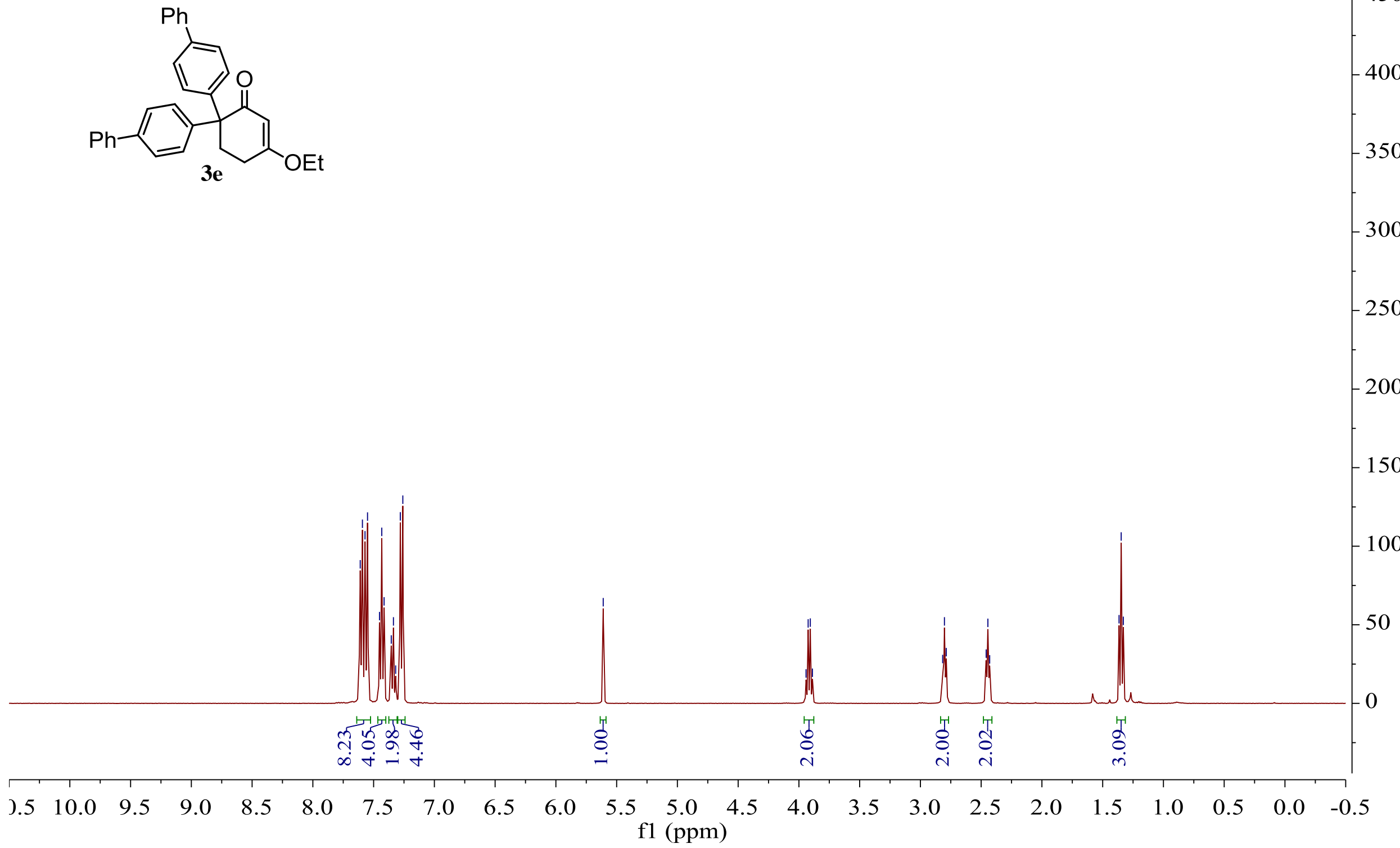
CARBON_01 -
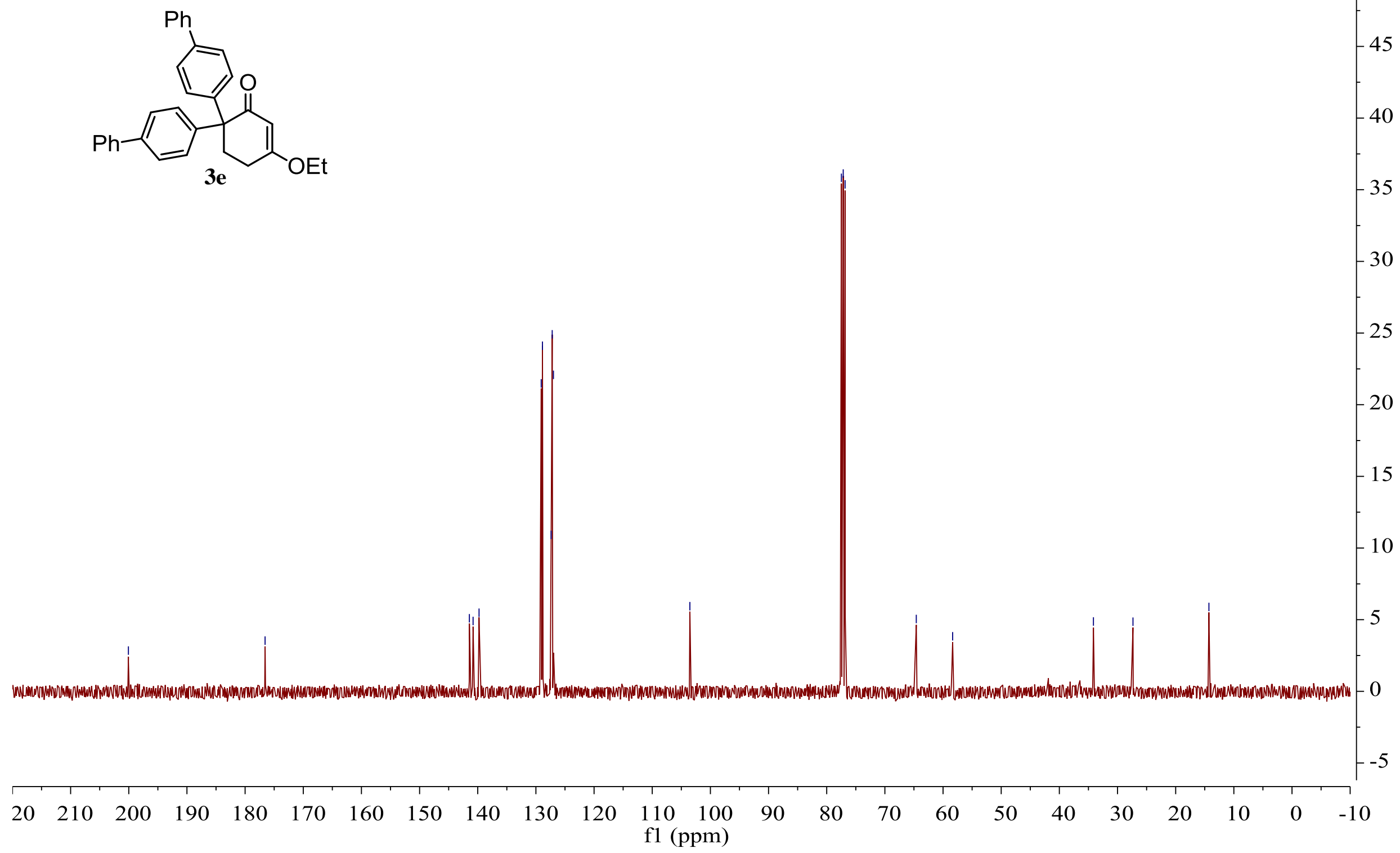
${ }^{1} \mathrm{H}$ NMR spectrum of compound $3 \mathrm{f}$

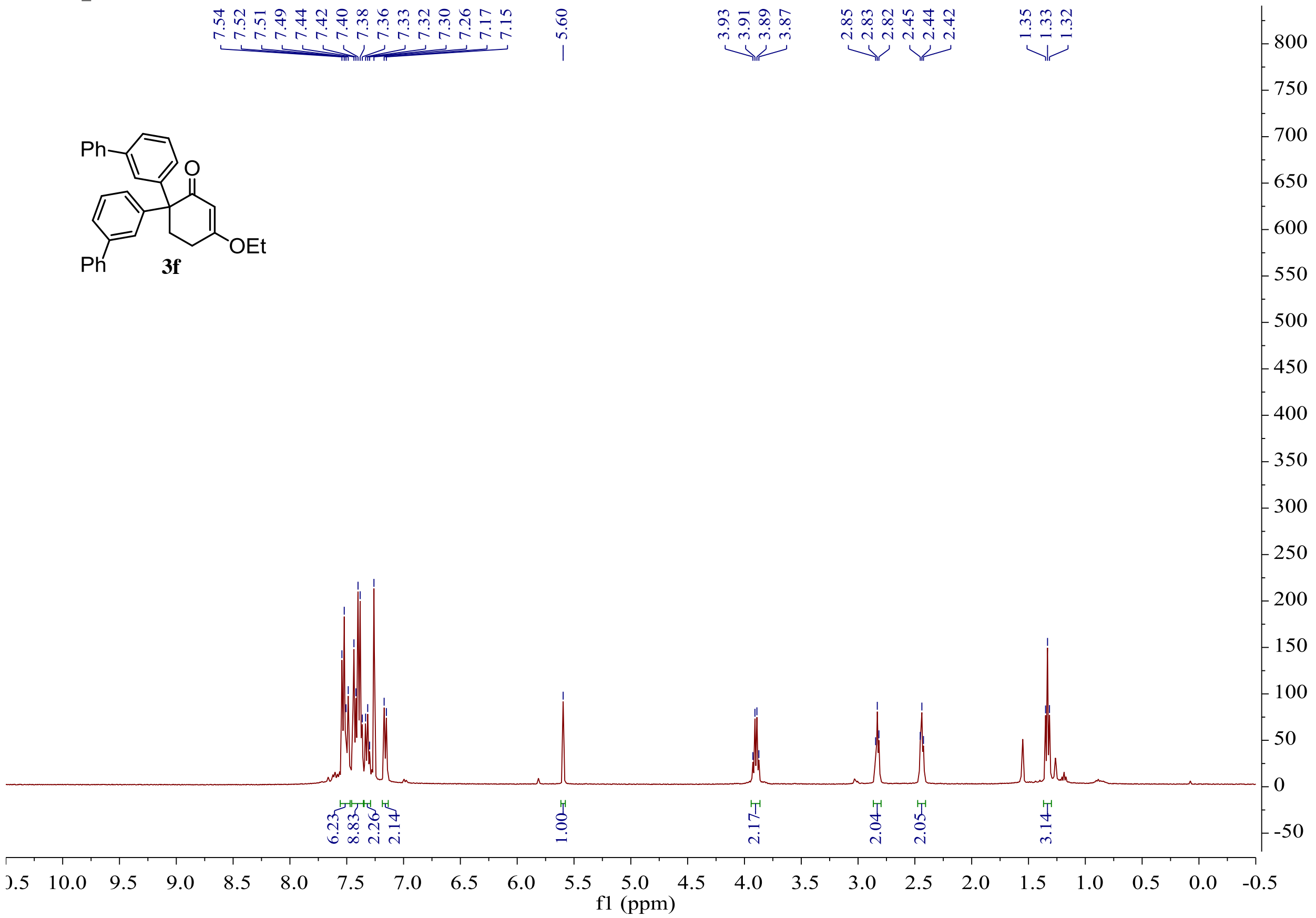


CARBON_01 -

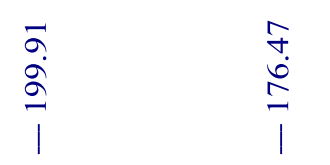

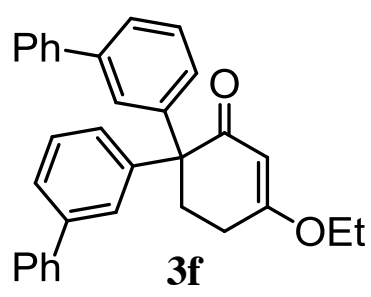

${ }^{13} \mathrm{C}$ NMR spectrum of compound $3 f$

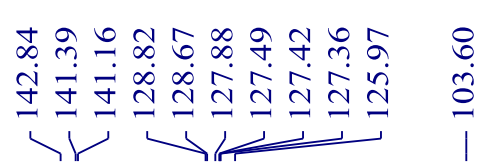

8
$\dot{0}$
0
1

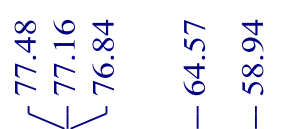




\section{${ }^{1} \mathrm{H}$ NMR spectrum of compound $3 \mathrm{~g}$}

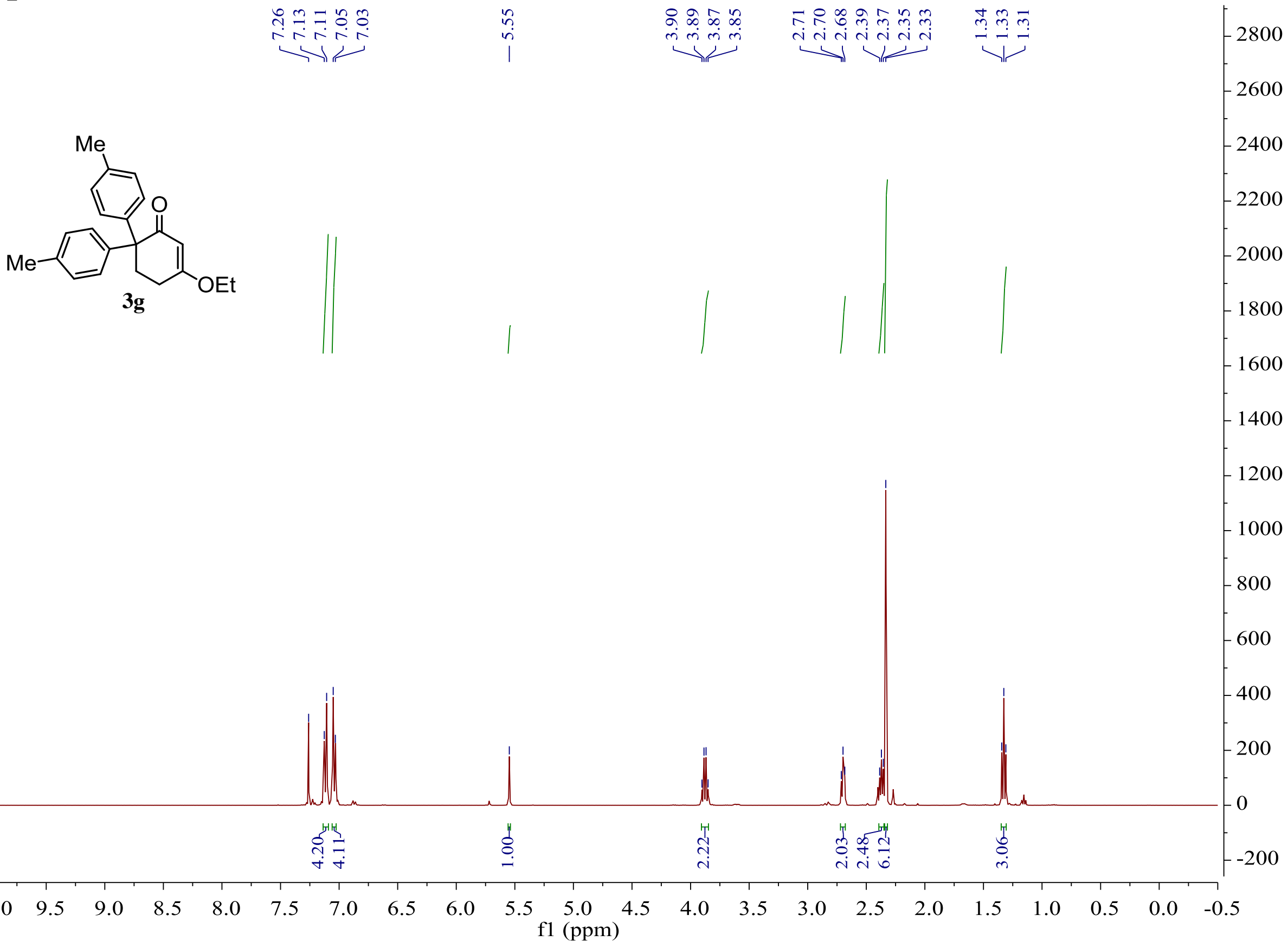


CARBON_01

${ }^{13} \mathrm{C}$ NMR spectrum of compound $3 \mathrm{~g}$
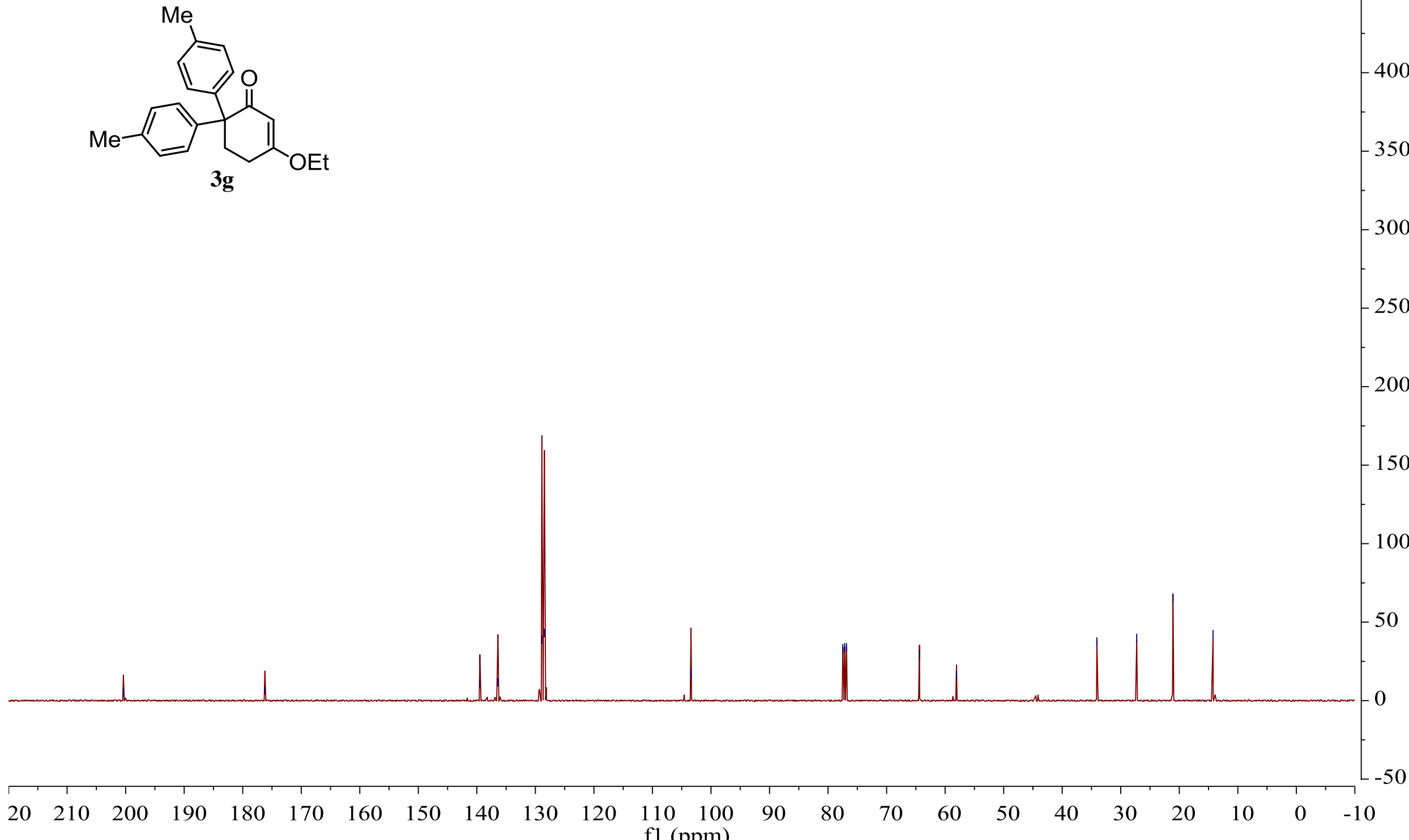


\section{${ }^{1} \mathrm{H}$ NMR spectrum of compound $3 \mathrm{~h}$}

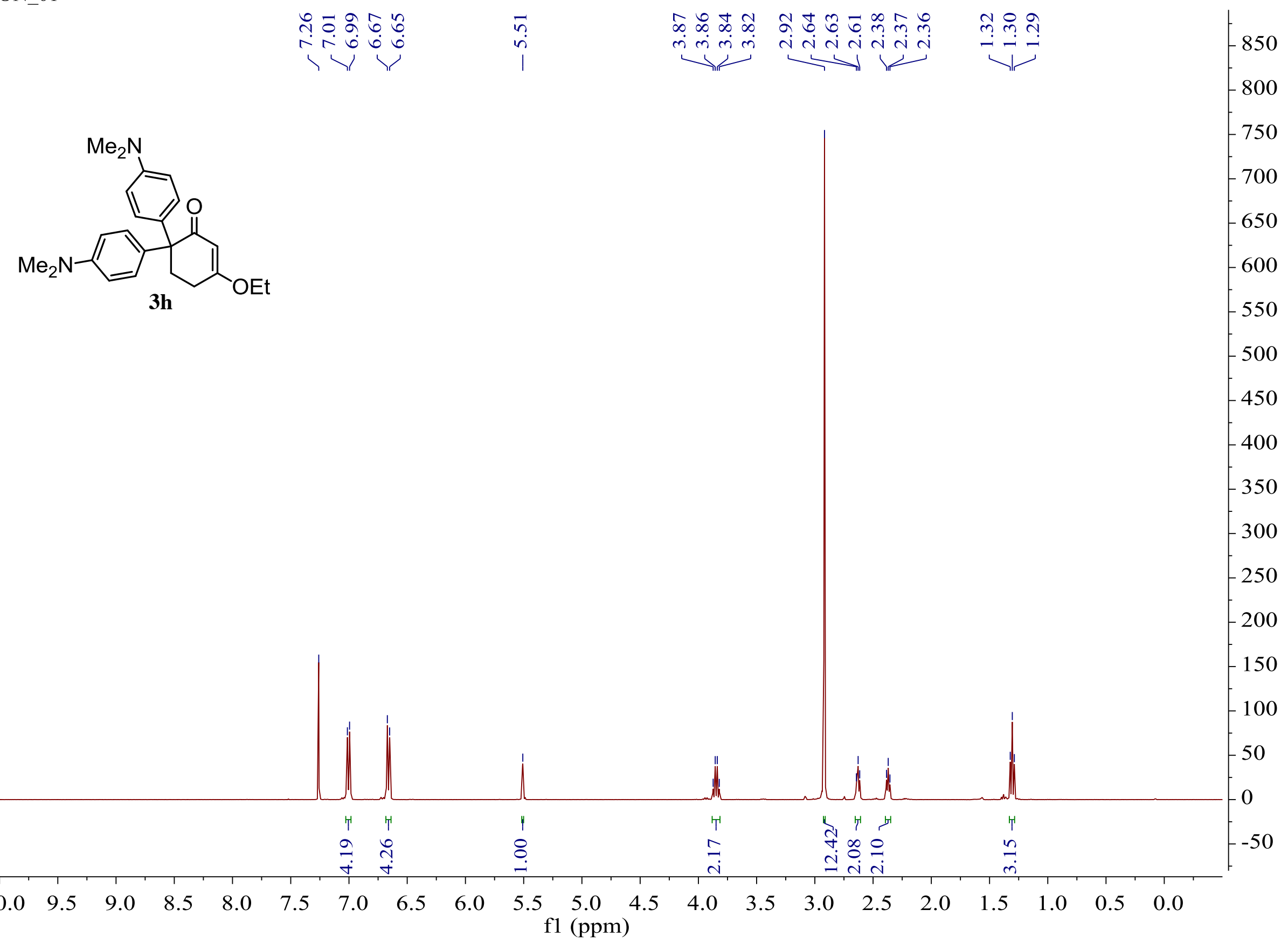


CARBON_01

${ }^{13} \mathrm{C}$ NMR spectrum of compound $3 \mathrm{~h}$
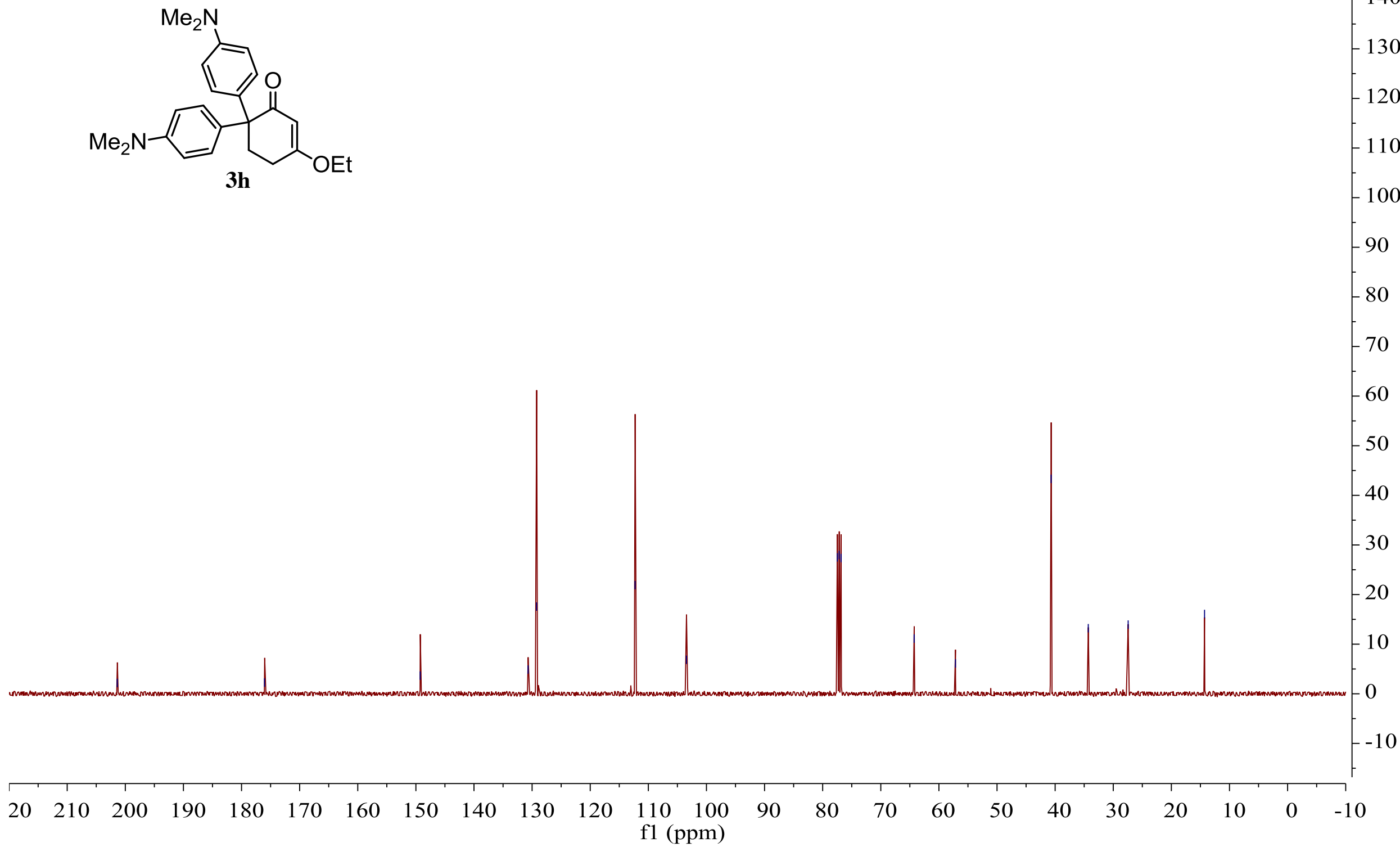


\section{${ }^{1} \mathrm{H}$ NMR spectrum of compound $3 \mathbf{i}$}

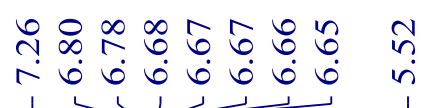

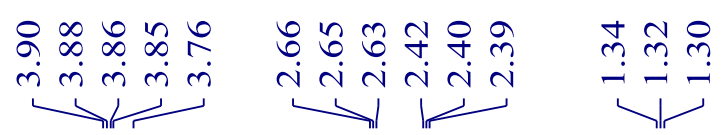
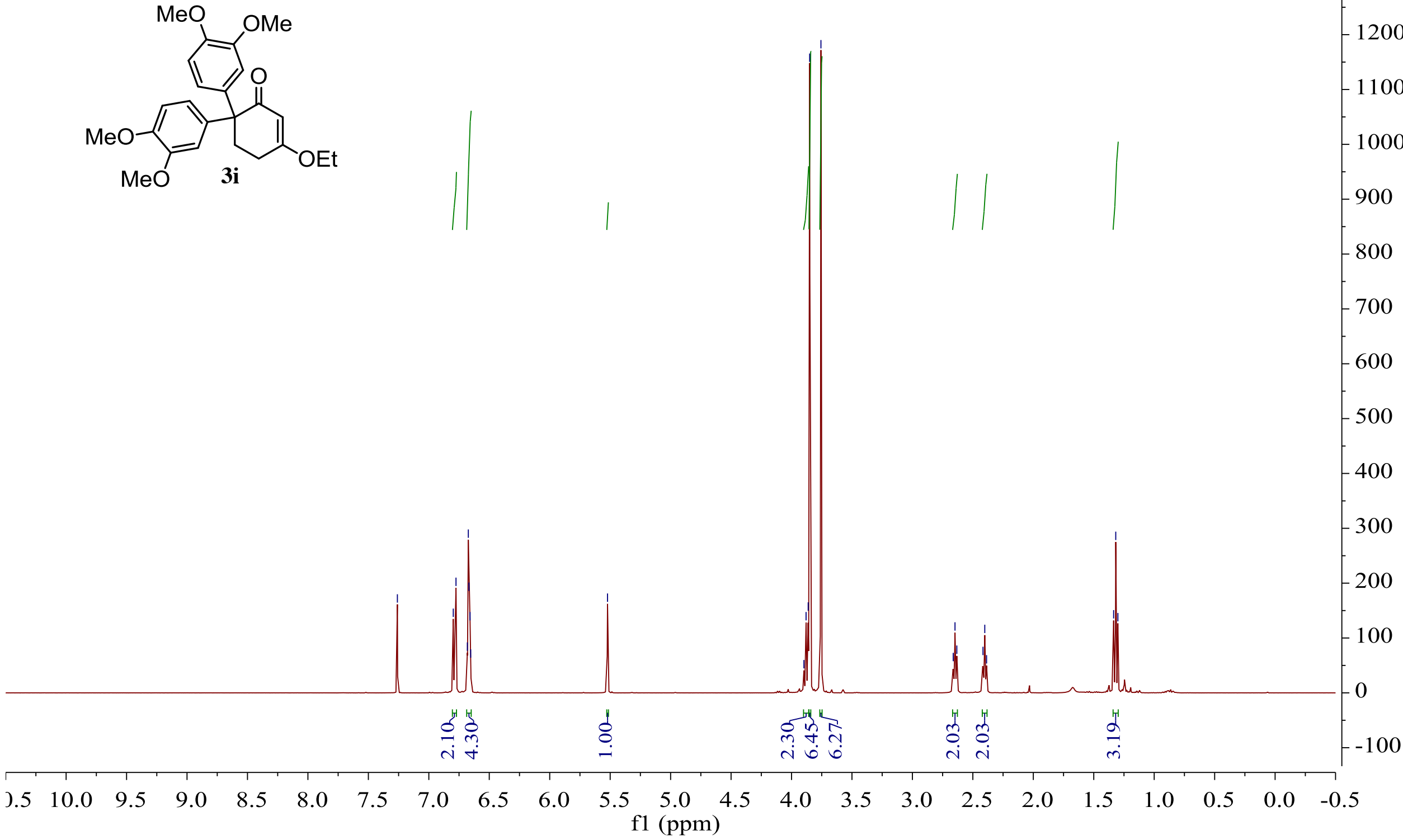
CARBON_01

${ }^{13} \mathrm{C}$ NMR spectrum of compound $3 i$

in

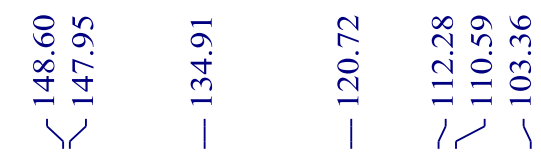

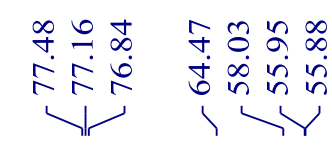

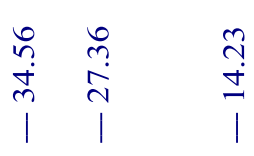

$-340$

$-320$

300
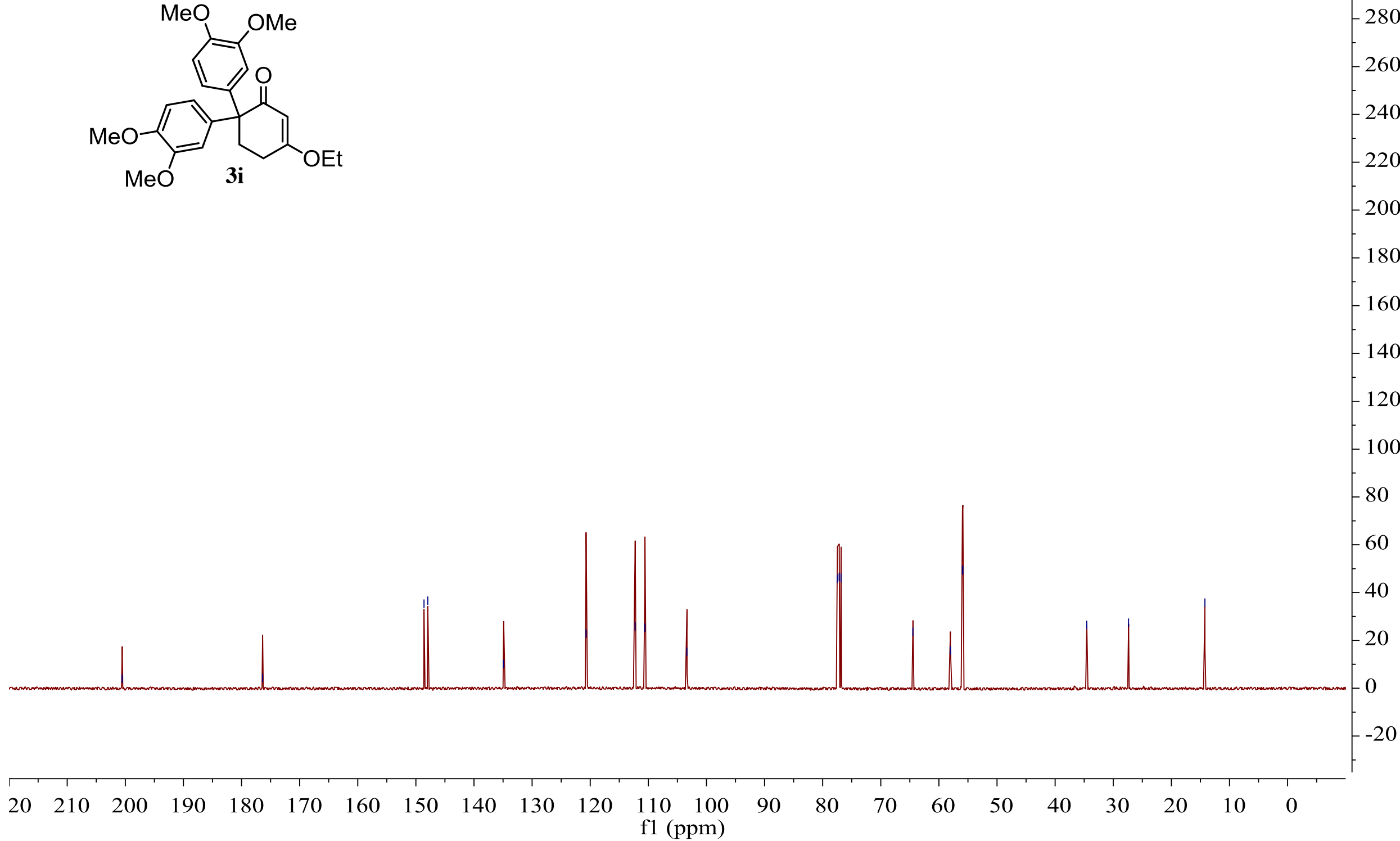


\section{${ }^{1} \mathbf{H}$ NMR spectrum of compound $3 \mathbf{j}$}

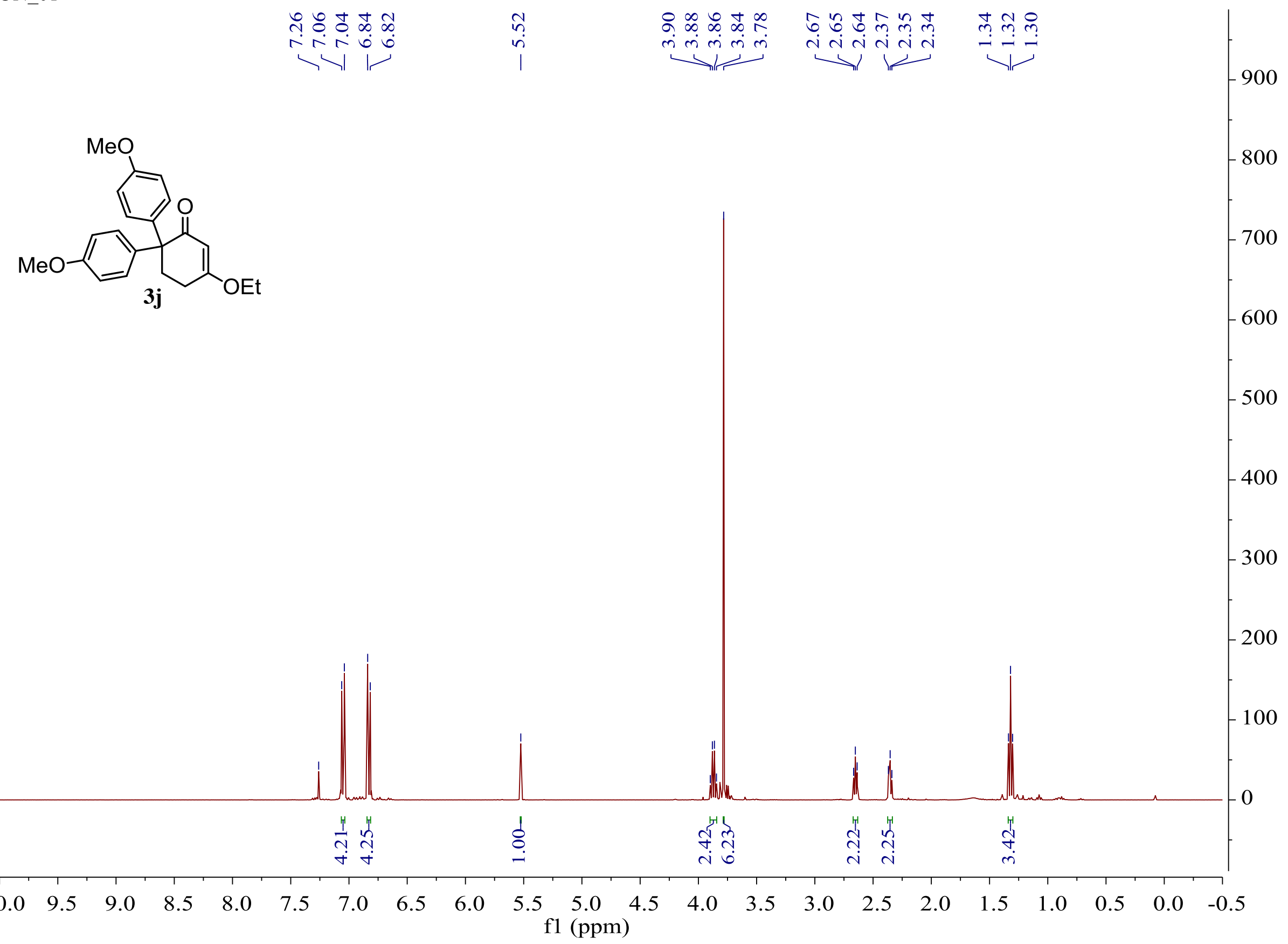


CARBON_01

${ }^{13} \mathrm{C}$ NMR spectrum of compound $3 \mathbf{j}$

官
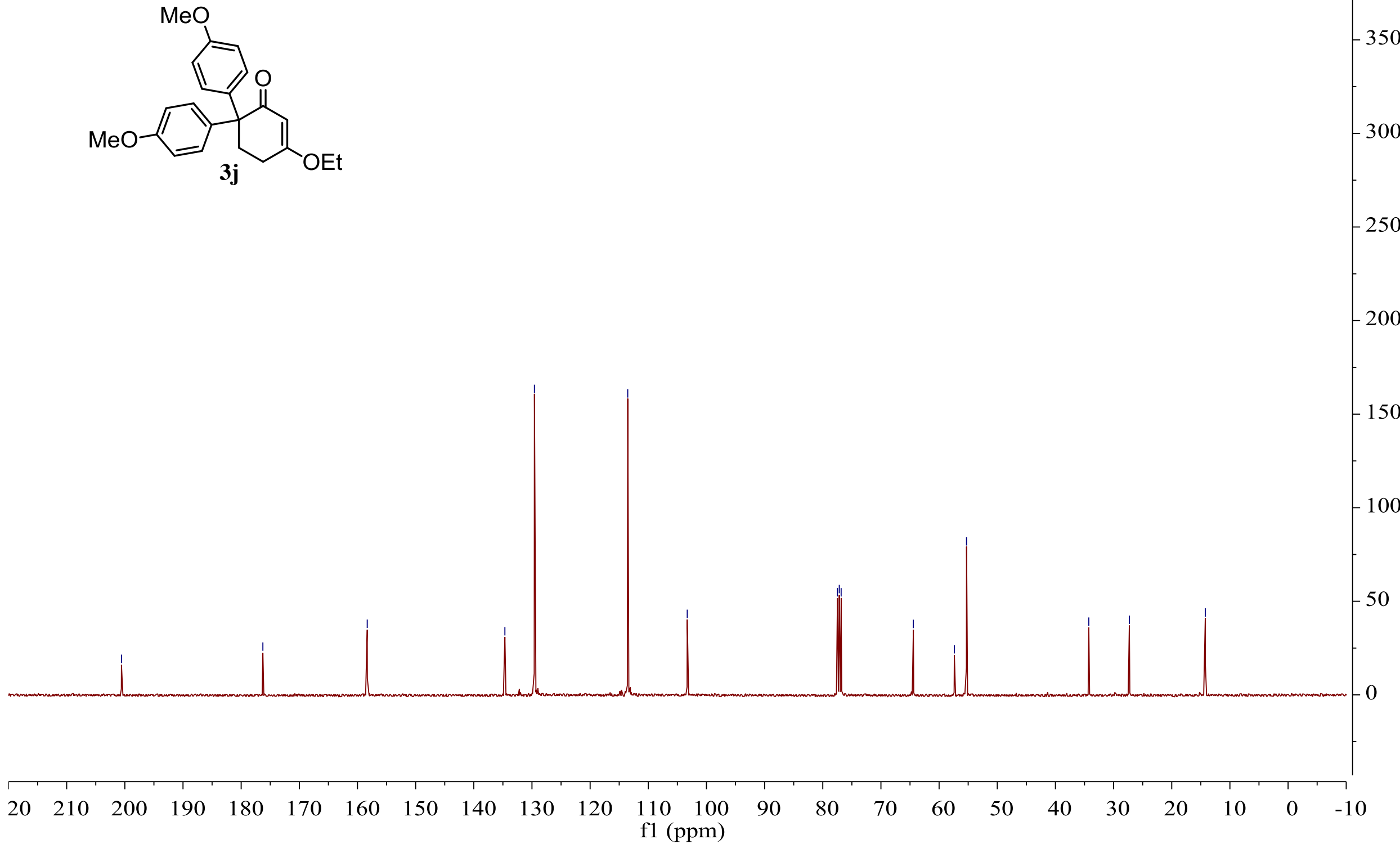
${ }^{1} \mathrm{H}$ NMR spectrum of compound $3 \mathrm{k}$

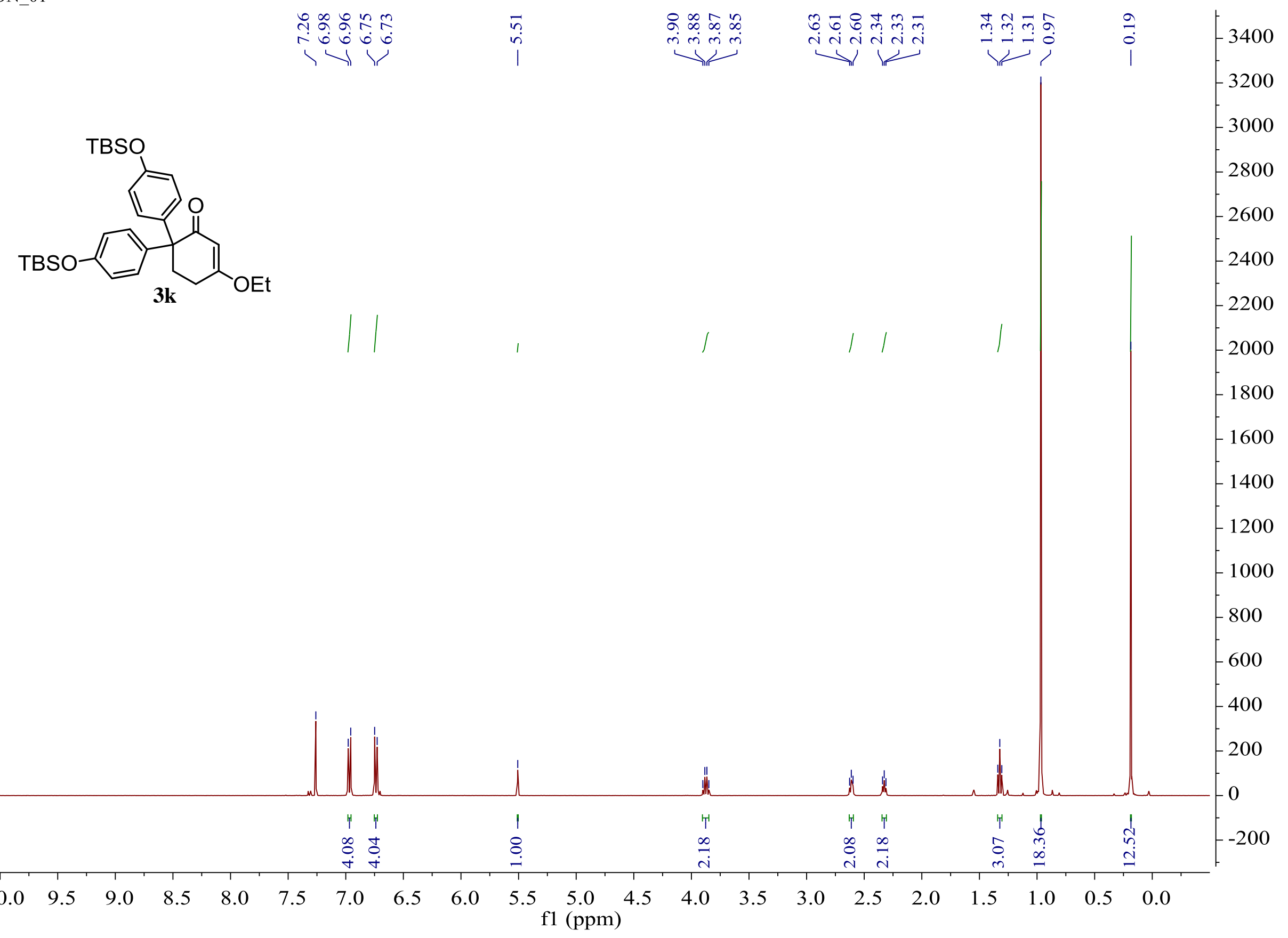




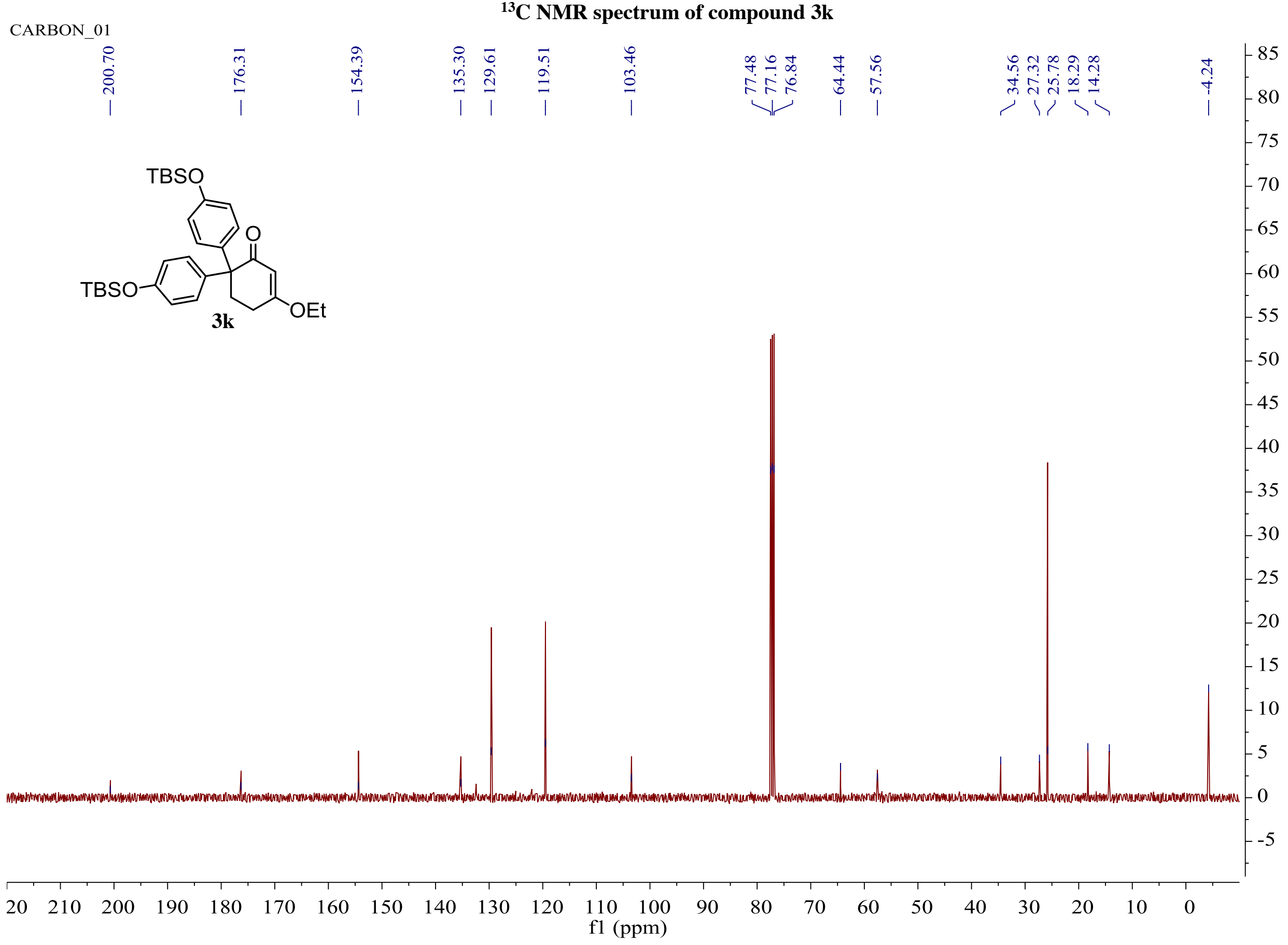




\section{${ }^{1} \mathbf{H}$ NMR spectrum of compound 31}

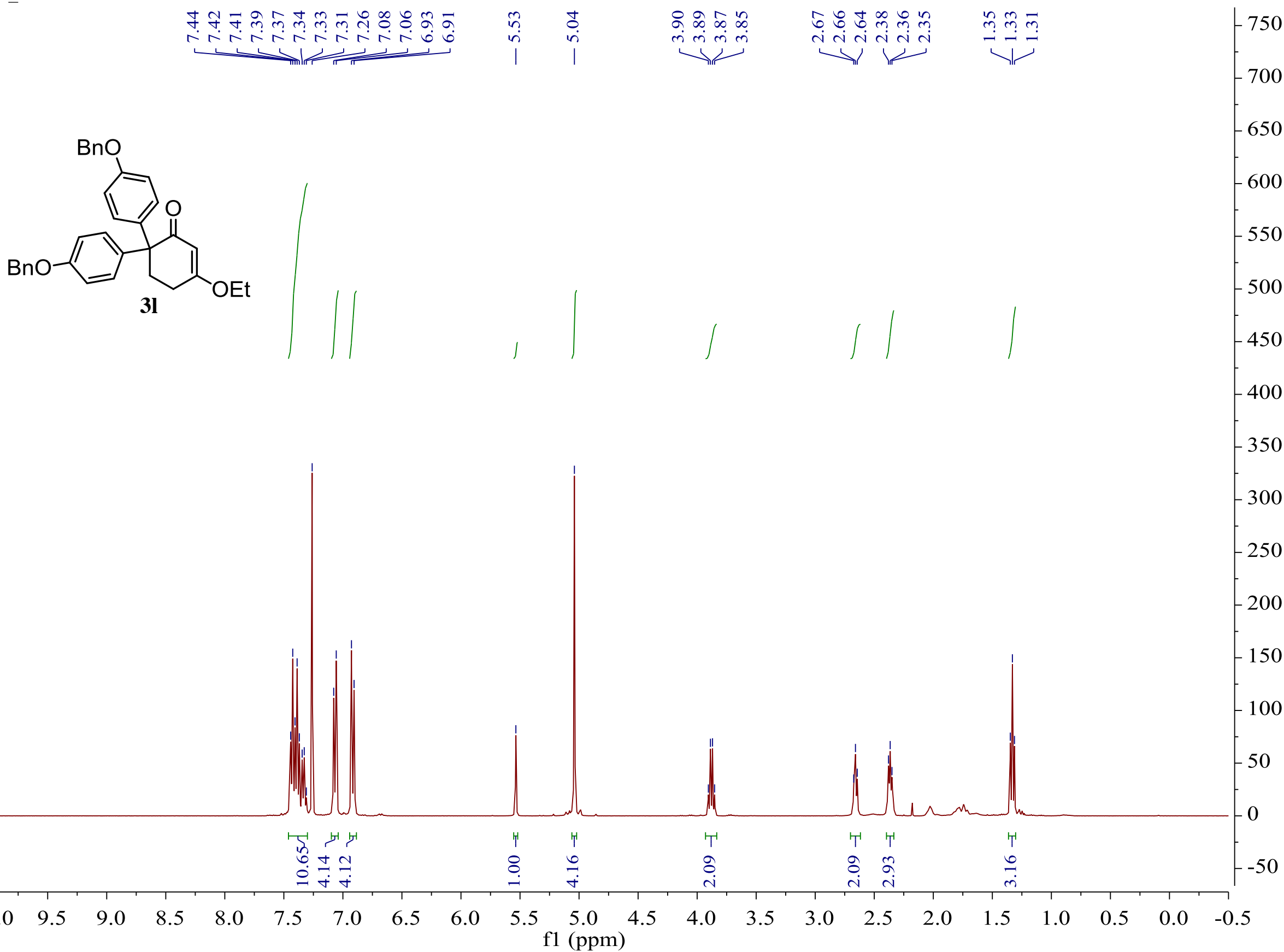


CARBON_01

${ }^{13} \mathrm{C}$ NMR spectrum of compound 31

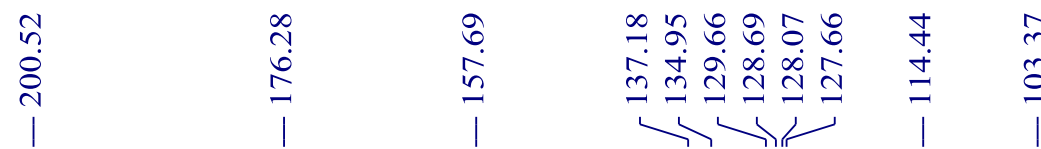

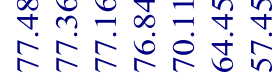

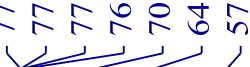

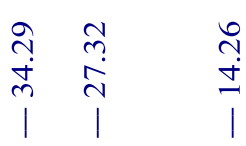

170

160

150

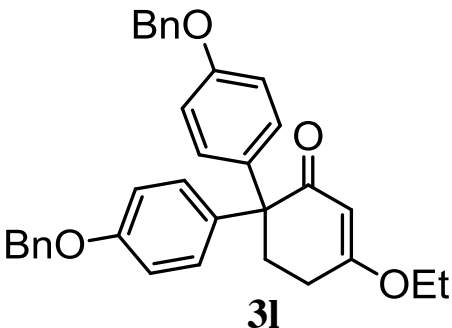

140

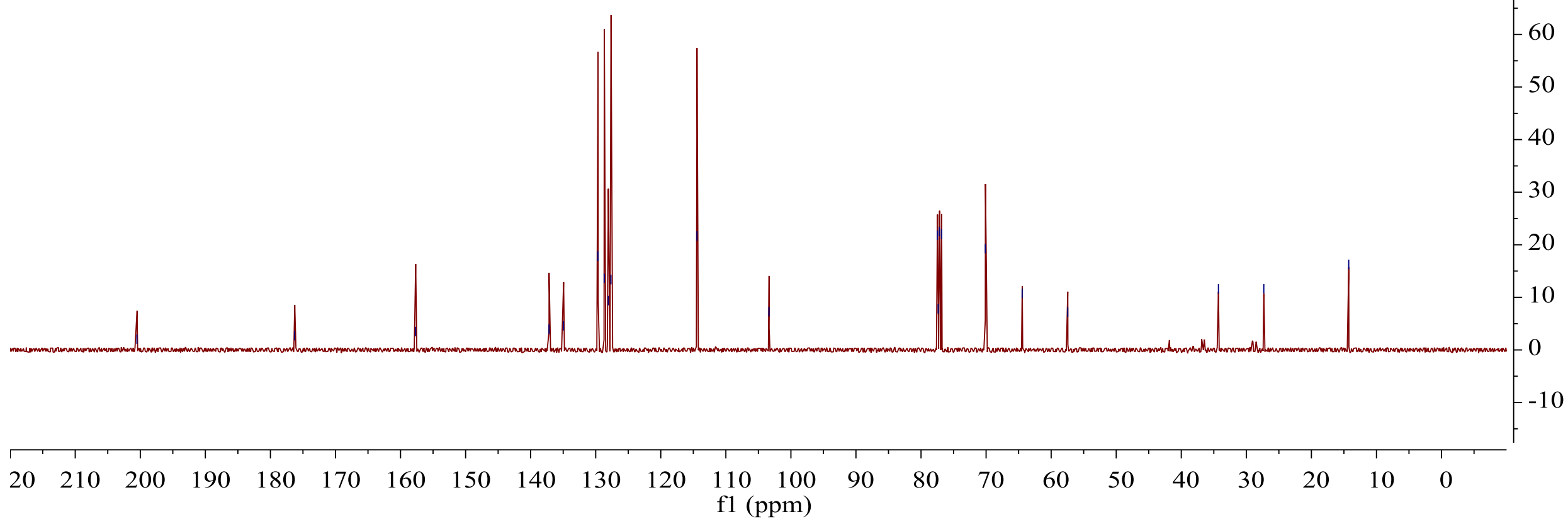


${ }^{1} \mathrm{H}$ NMR spectrum of compound $3 \mathrm{~m}$

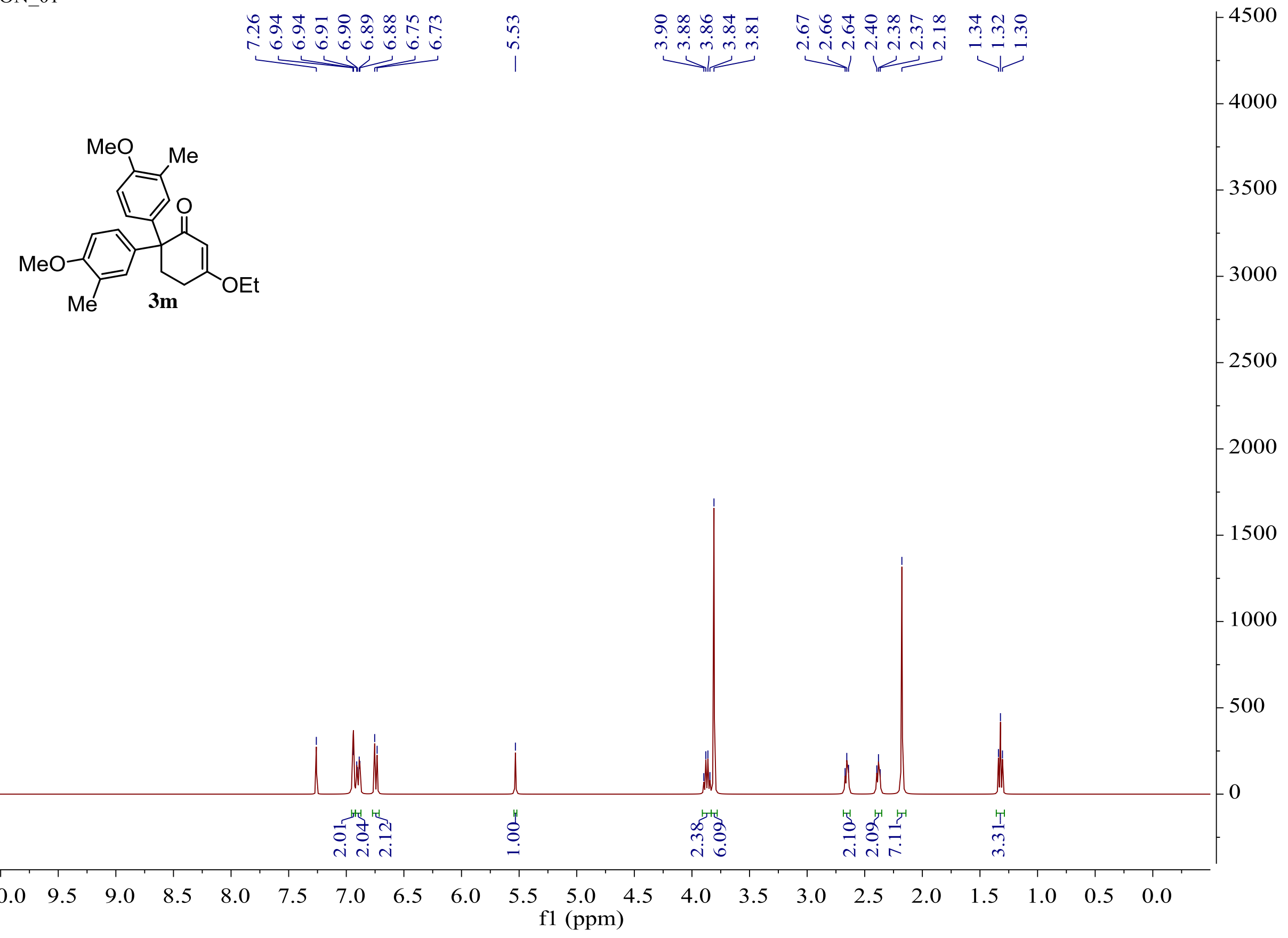




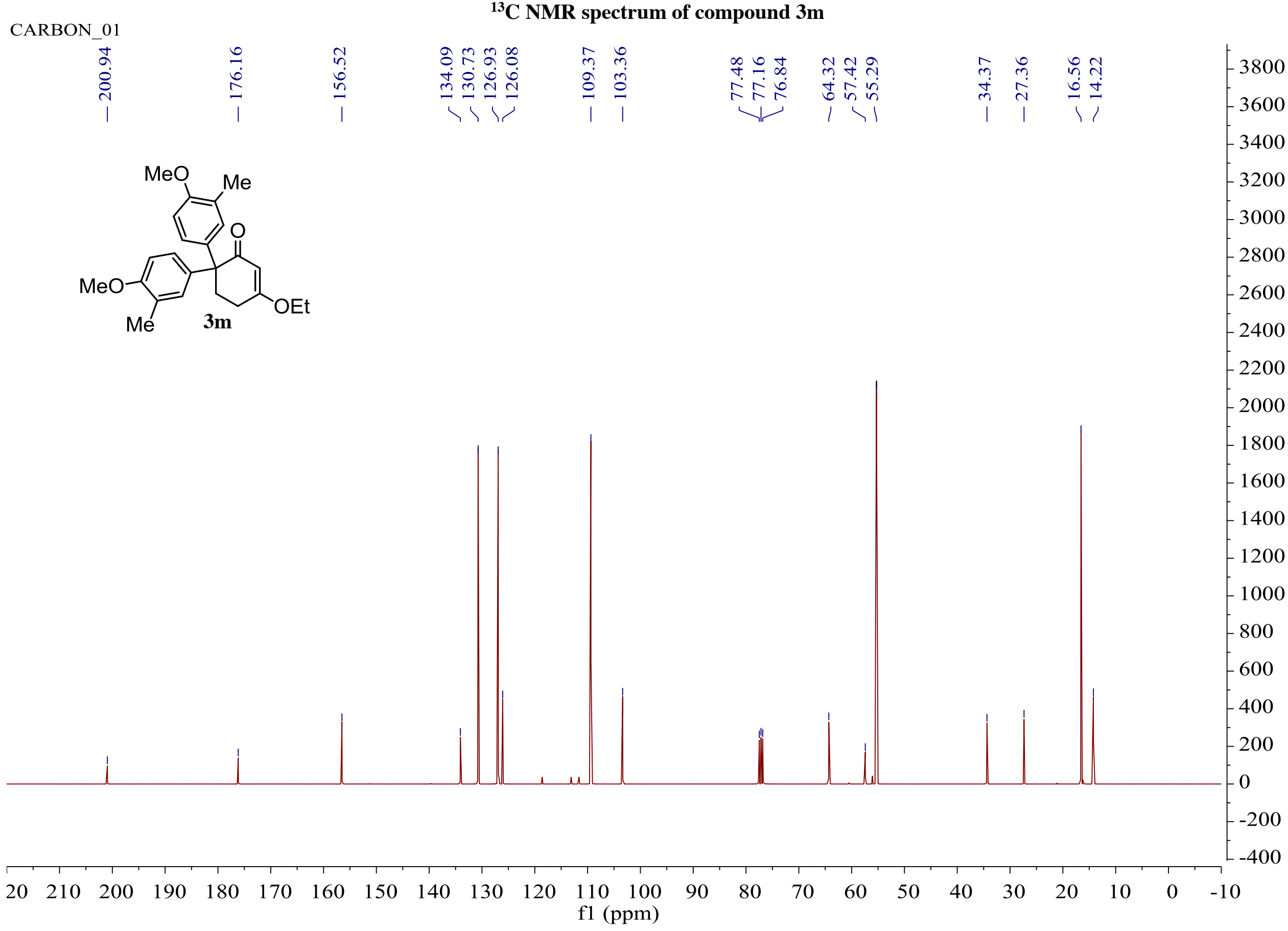


${ }^{1}$ H NMR spectrum of compound $3 n$

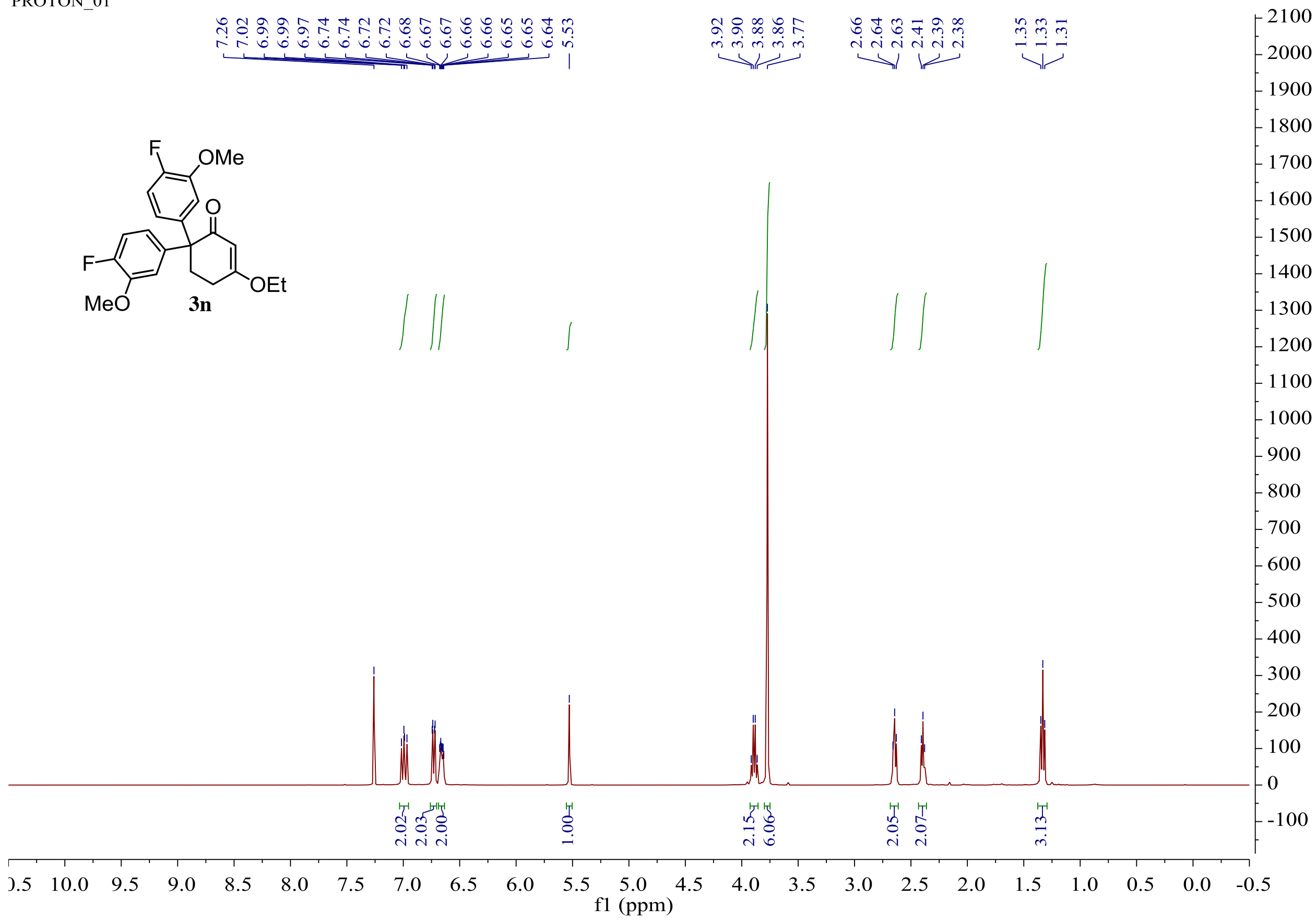


CARBON 01

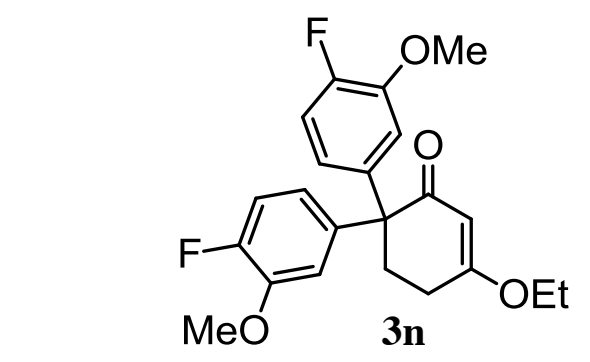

${ }^{13} \mathrm{C}$ NMR spectrum of compound $3 n$

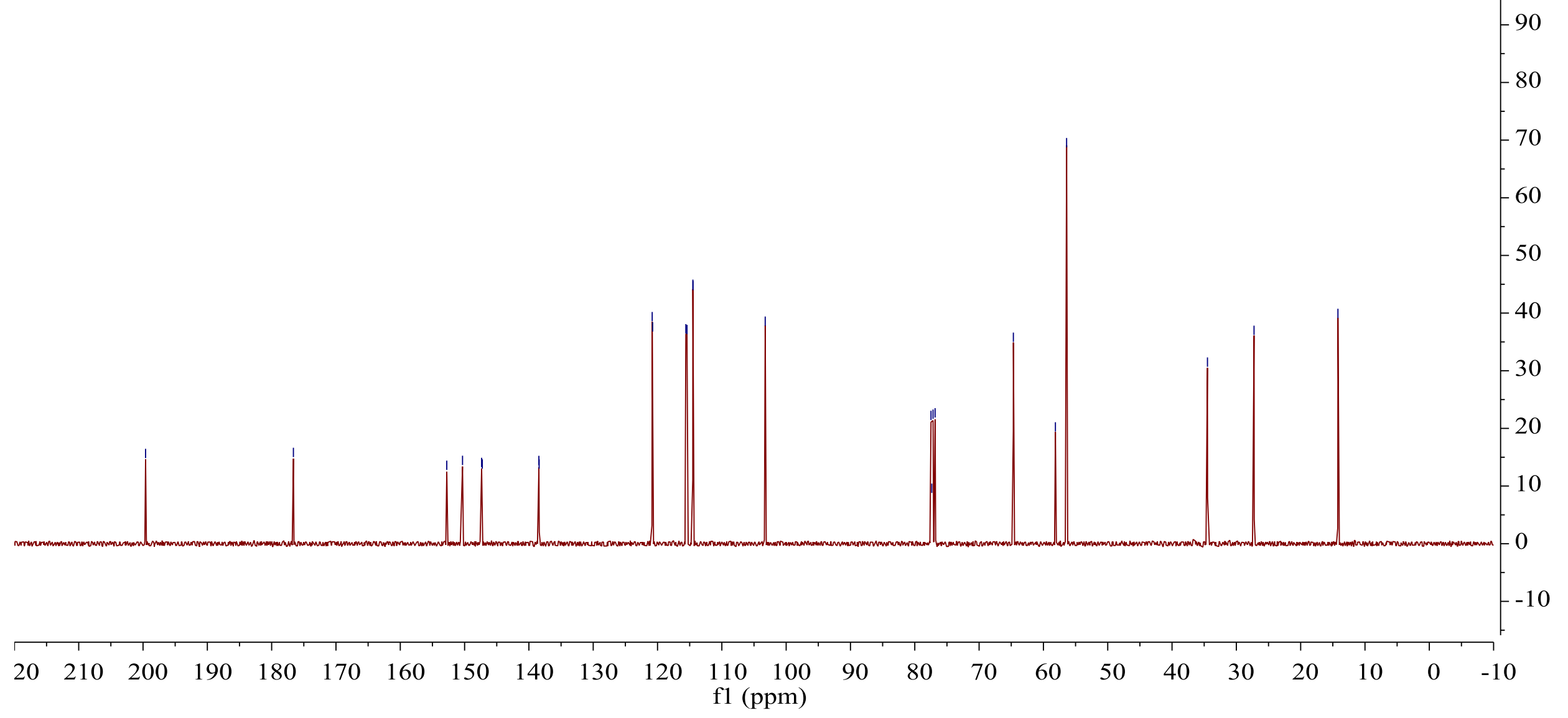


FLUORINE 01

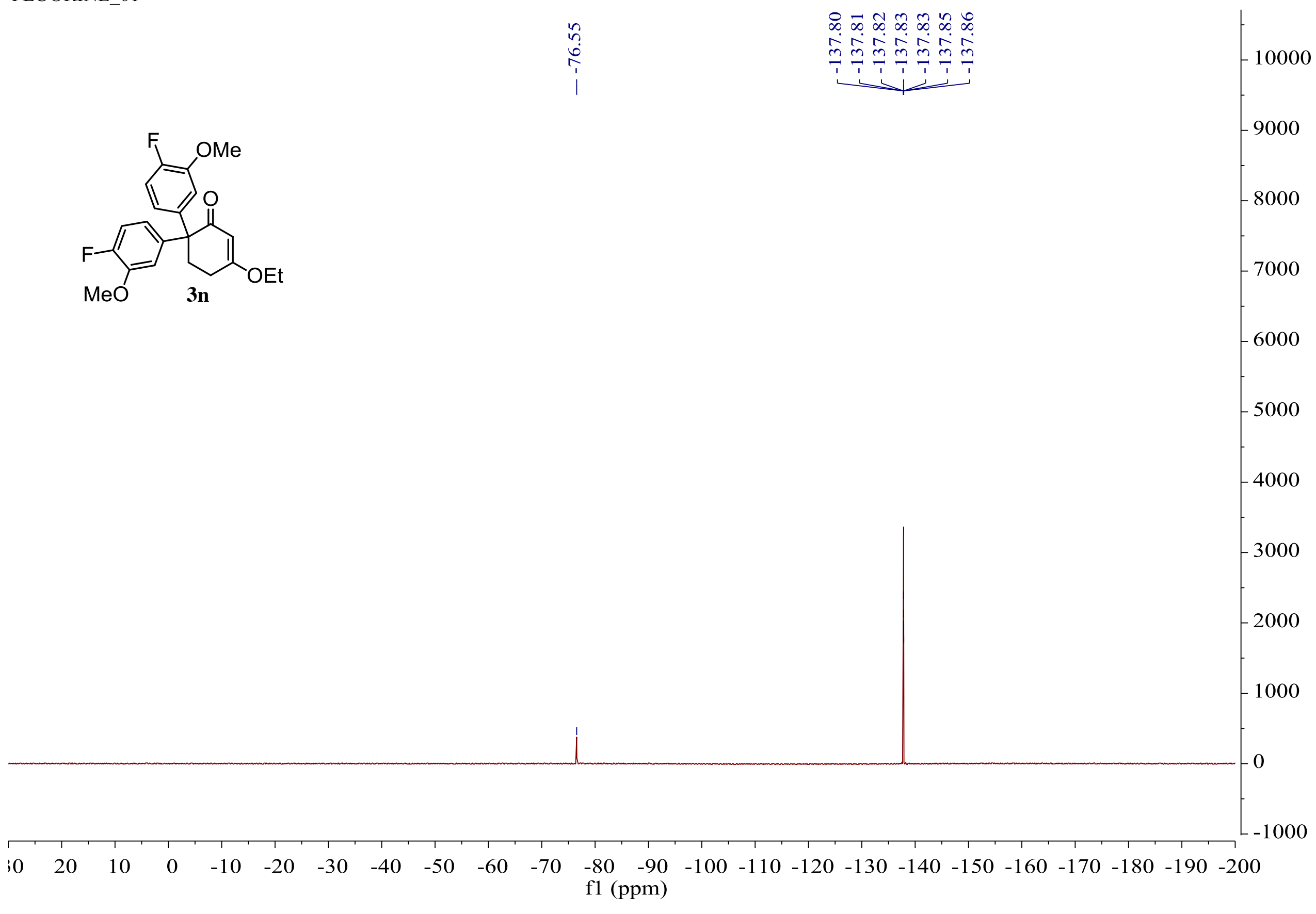

${ }^{19}$ F NMR spectrum of compound $3 n$ 


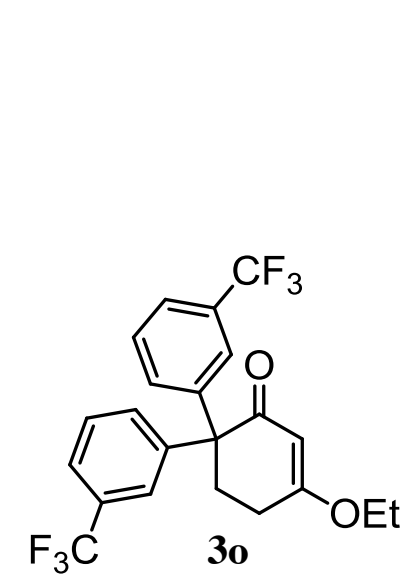

\section{${ }^{1} \mathrm{H}$ NMR spectrum of compound 30}

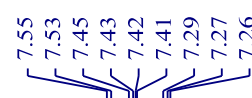

iे

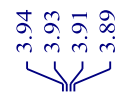

$\underbrace{\infty}$

|خ

3400

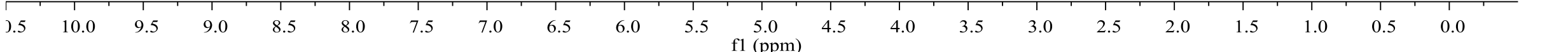




\section{${ }^{13} \mathrm{C}$ NMR spectrum of compound 30}

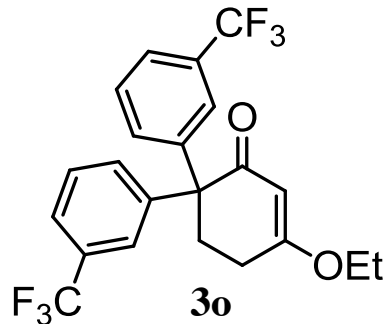




\section{${ }^{19}$ F NMR spectrum of compound 30}

$$
\begin{array}{ll}
2 & n \\
\infty & n \\
n & 0 \\
0 & n \\
1 & 1
\end{array}
$$
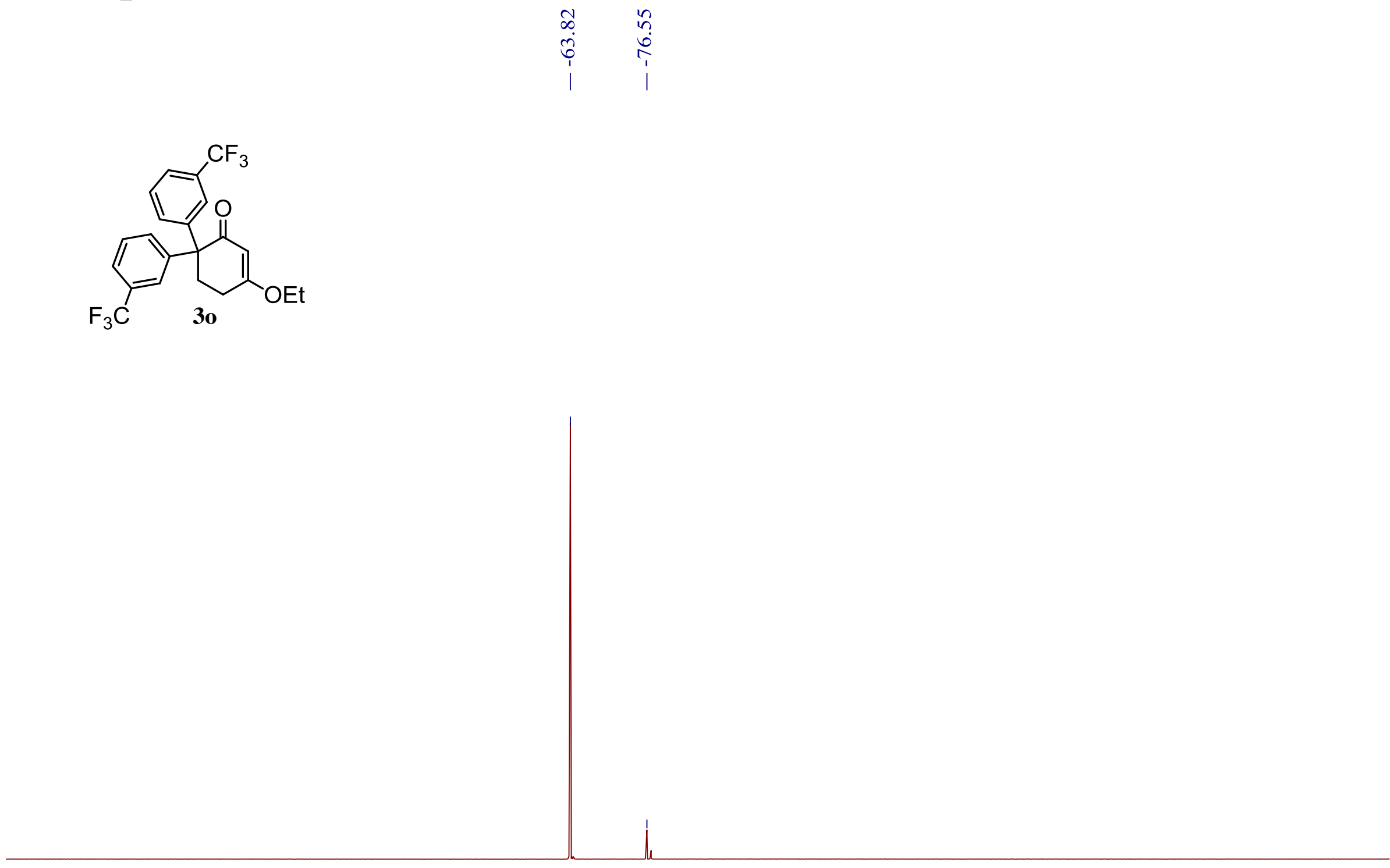


\section{${ }^{1} \mathrm{H}$ NMR spectrum of compound $3 p$}

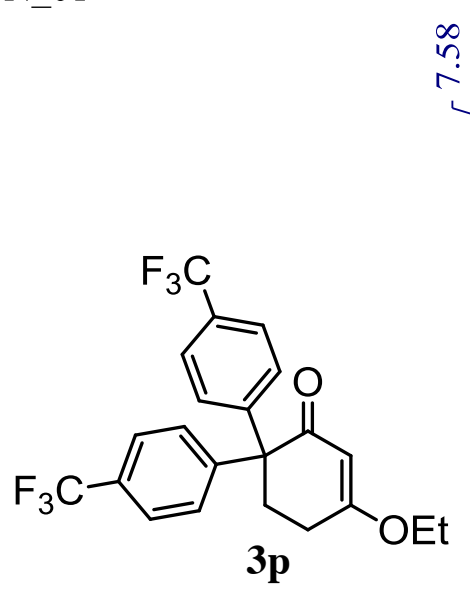

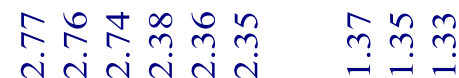

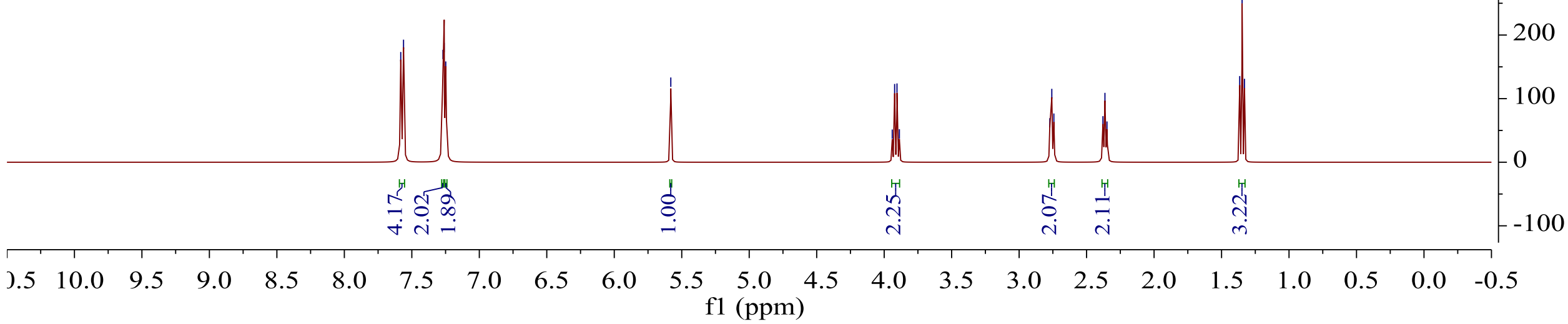


CARBON_01

${ }^{13} \mathrm{C}$ NMR spectrum of compound $3 p$

\begin{tabular}{l}
\multirow{2}{*}{} \\
$\infty$ \\
$\stackrel{\infty}{2}$
\end{tabular}

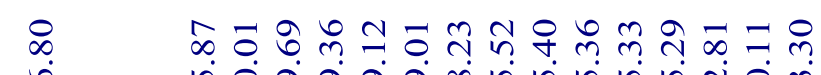

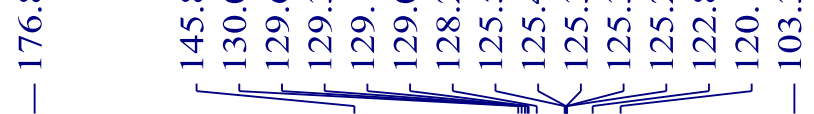

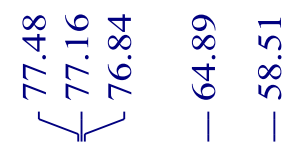

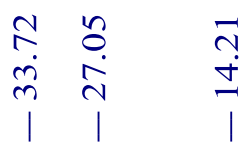
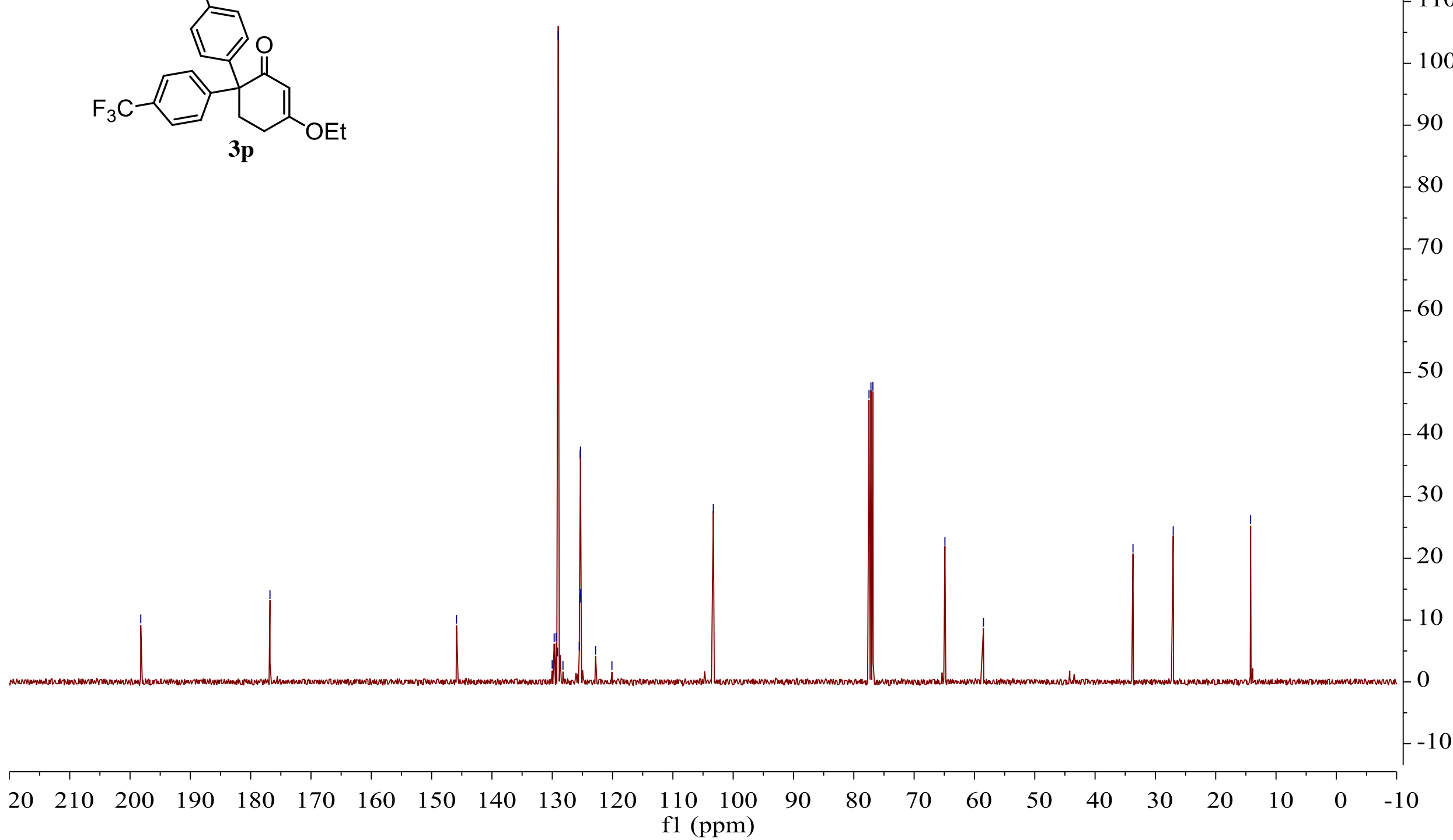


\section{${ }^{19}$ F NMR spectrum of compound 3p}

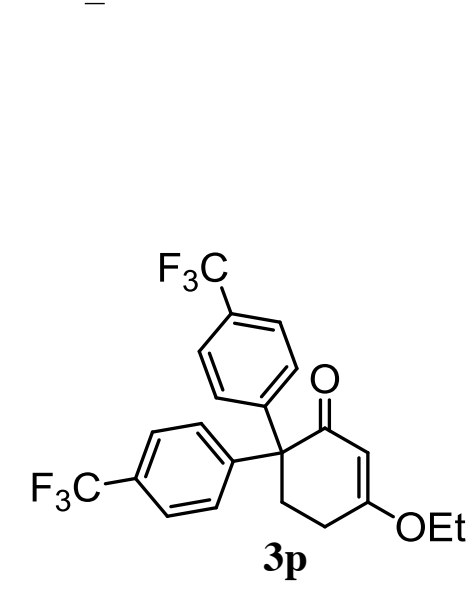


PROTON_01

लै

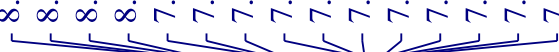

\section{${ }^{1} \mathrm{H}$ NMR spectrum of compound $3 q$}

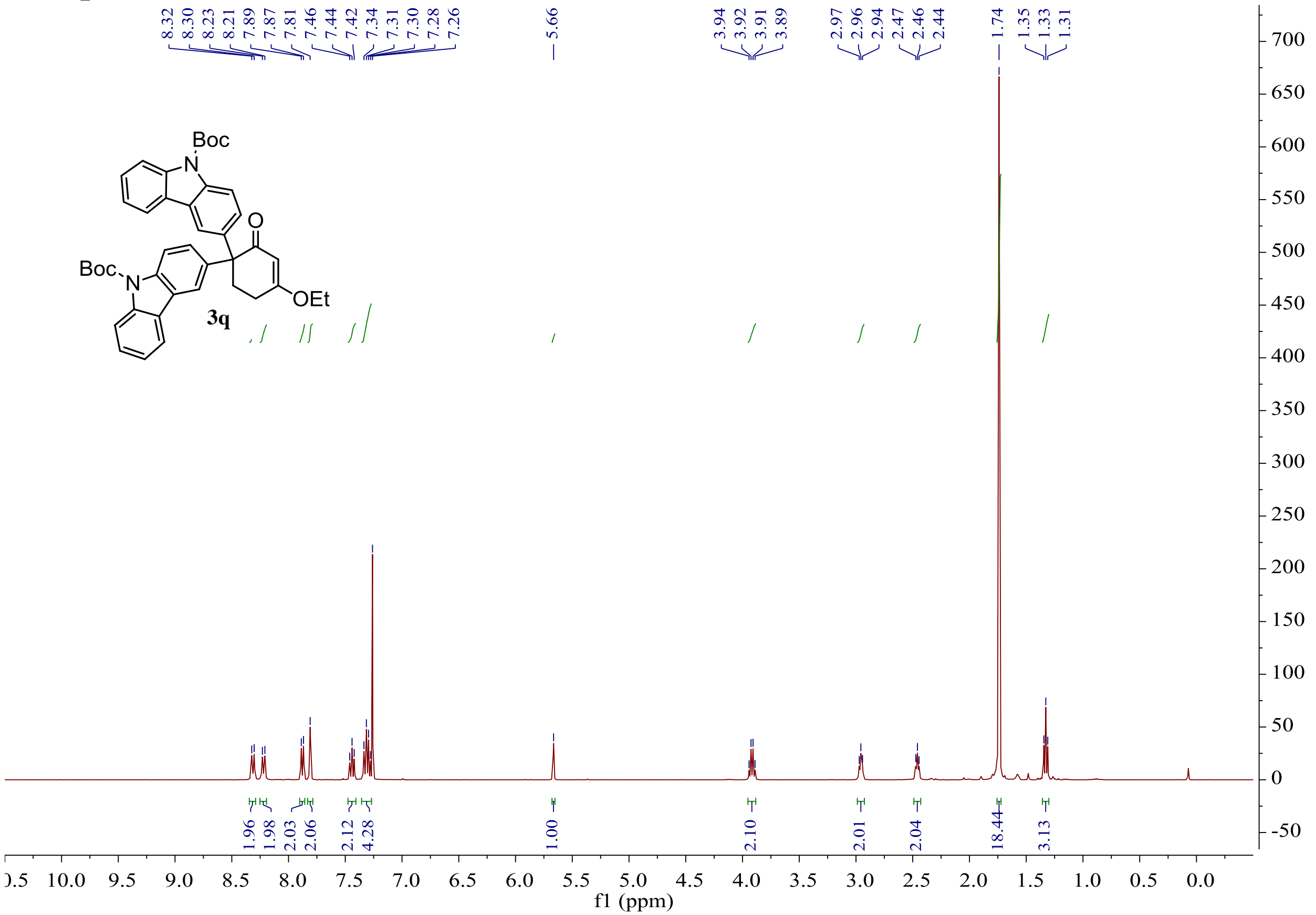




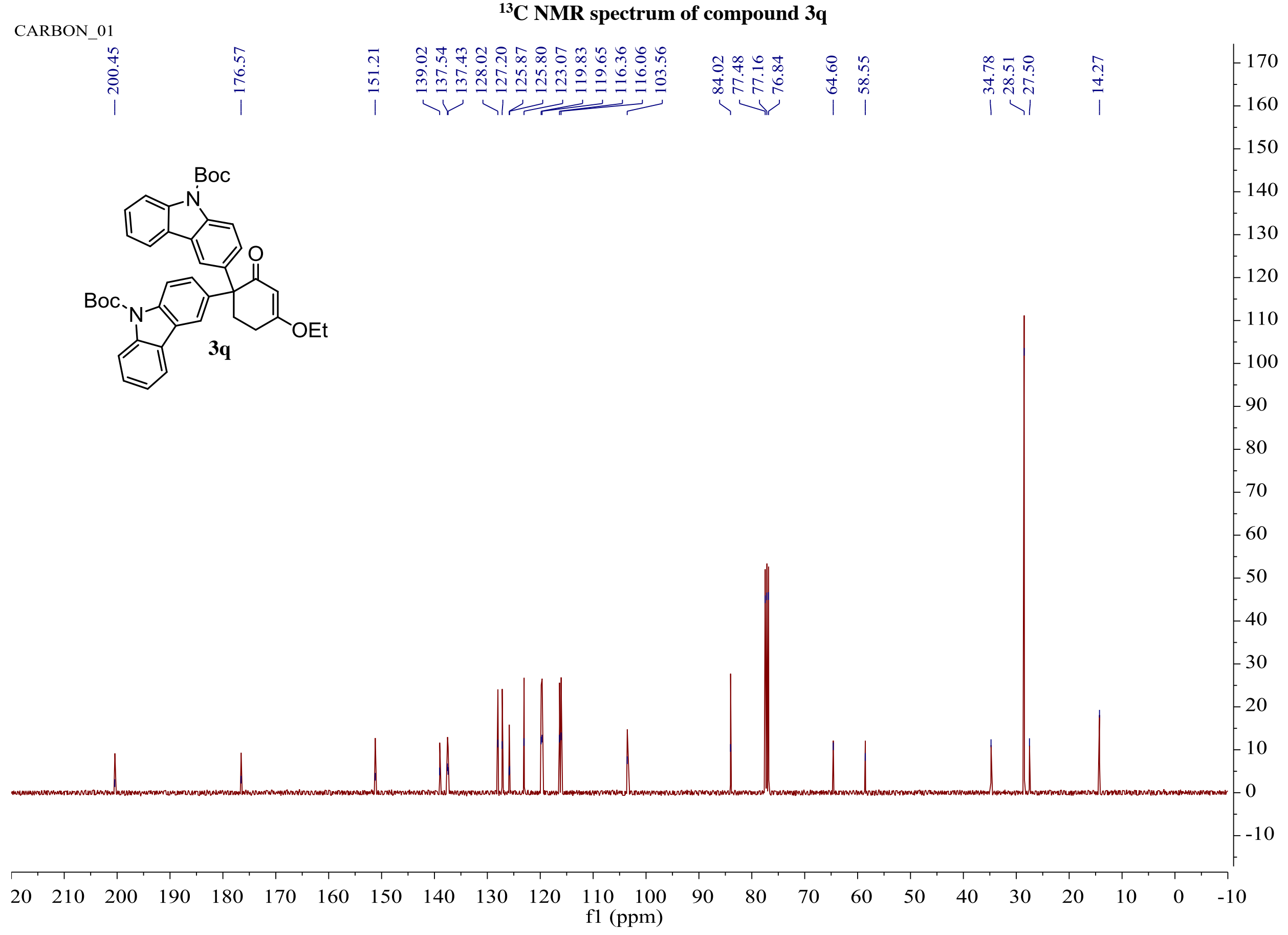




\section{${ }^{1} \mathrm{H}$ NMR spectrum of compound $3 \mathbf{r}$}

๓ุ

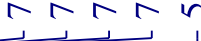

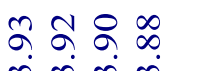

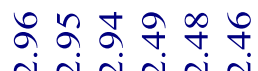

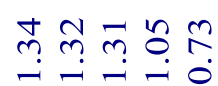

4 N

$-550$

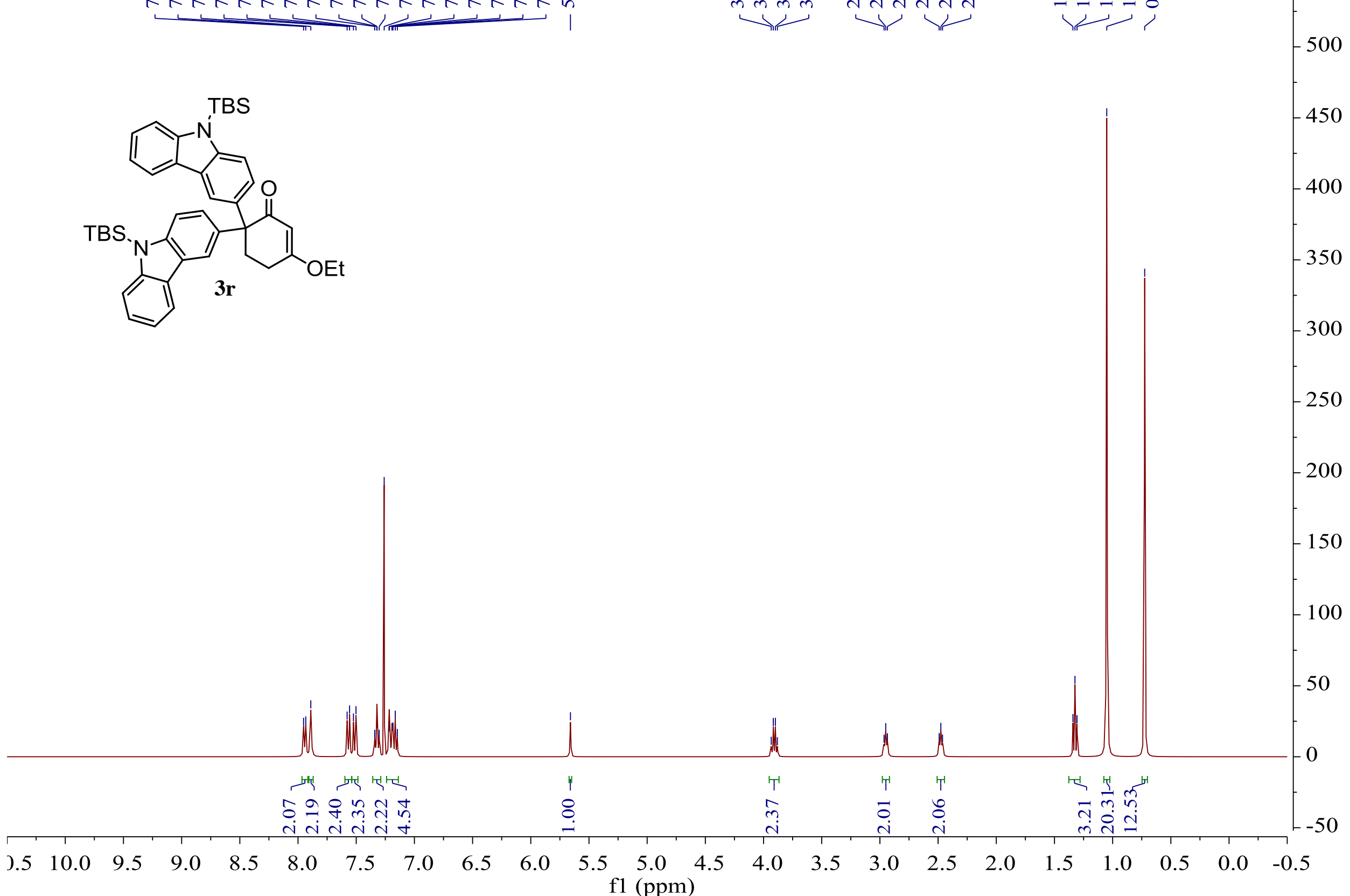




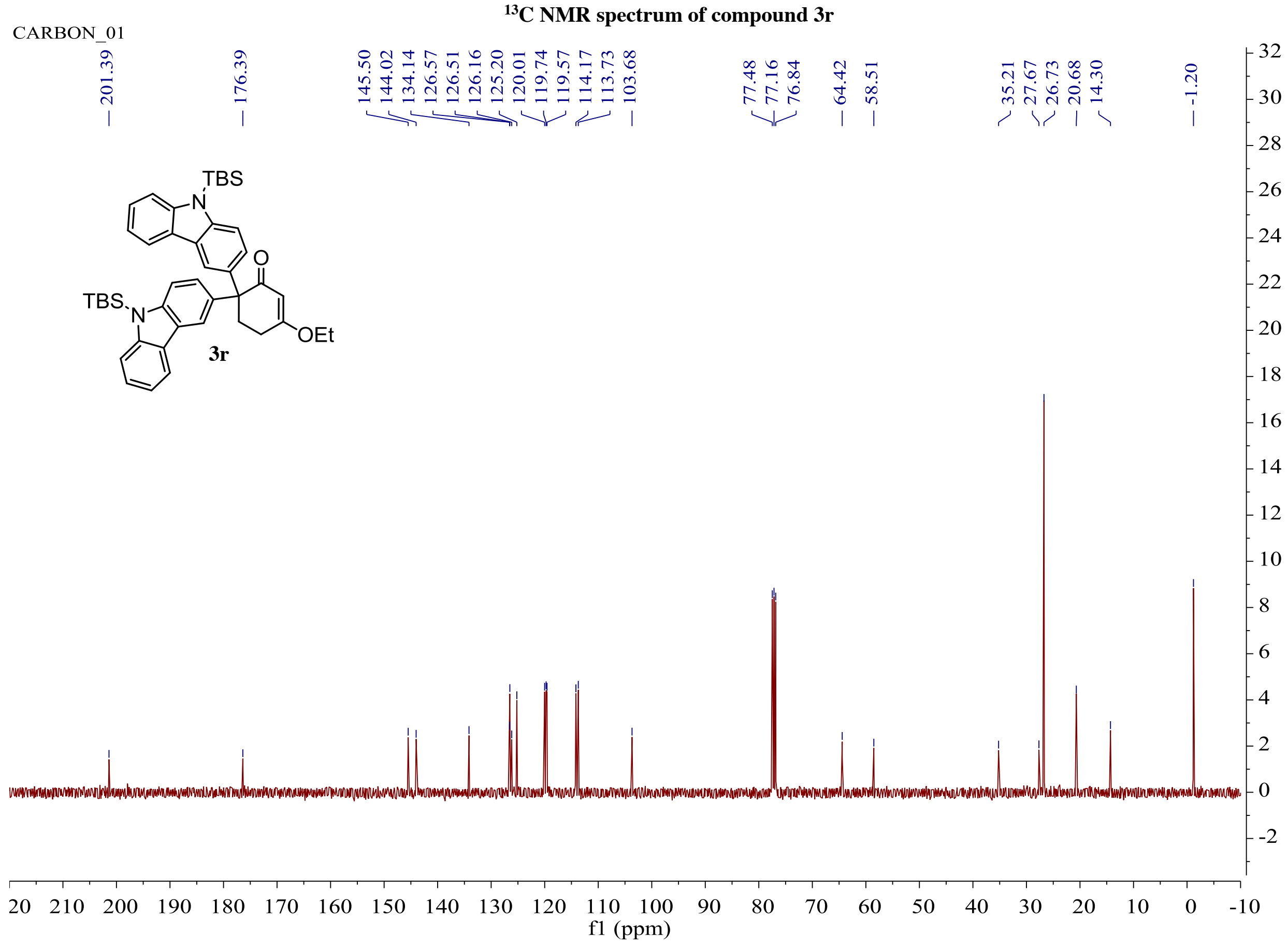




\section{${ }^{1} \mathrm{H}$ NMR spectrum of compound $3 \mathrm{~s}$}

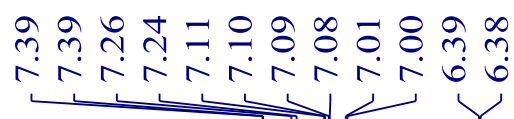

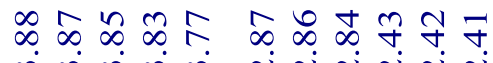

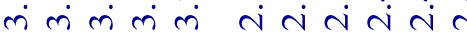

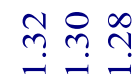

$\longrightarrow$

可<smiles>CCOC1=CC(=O)C(c2ccc3c(ccn3C)c2)(c2ccc3c(ccn3C)c2)CC1</smiles>

3s

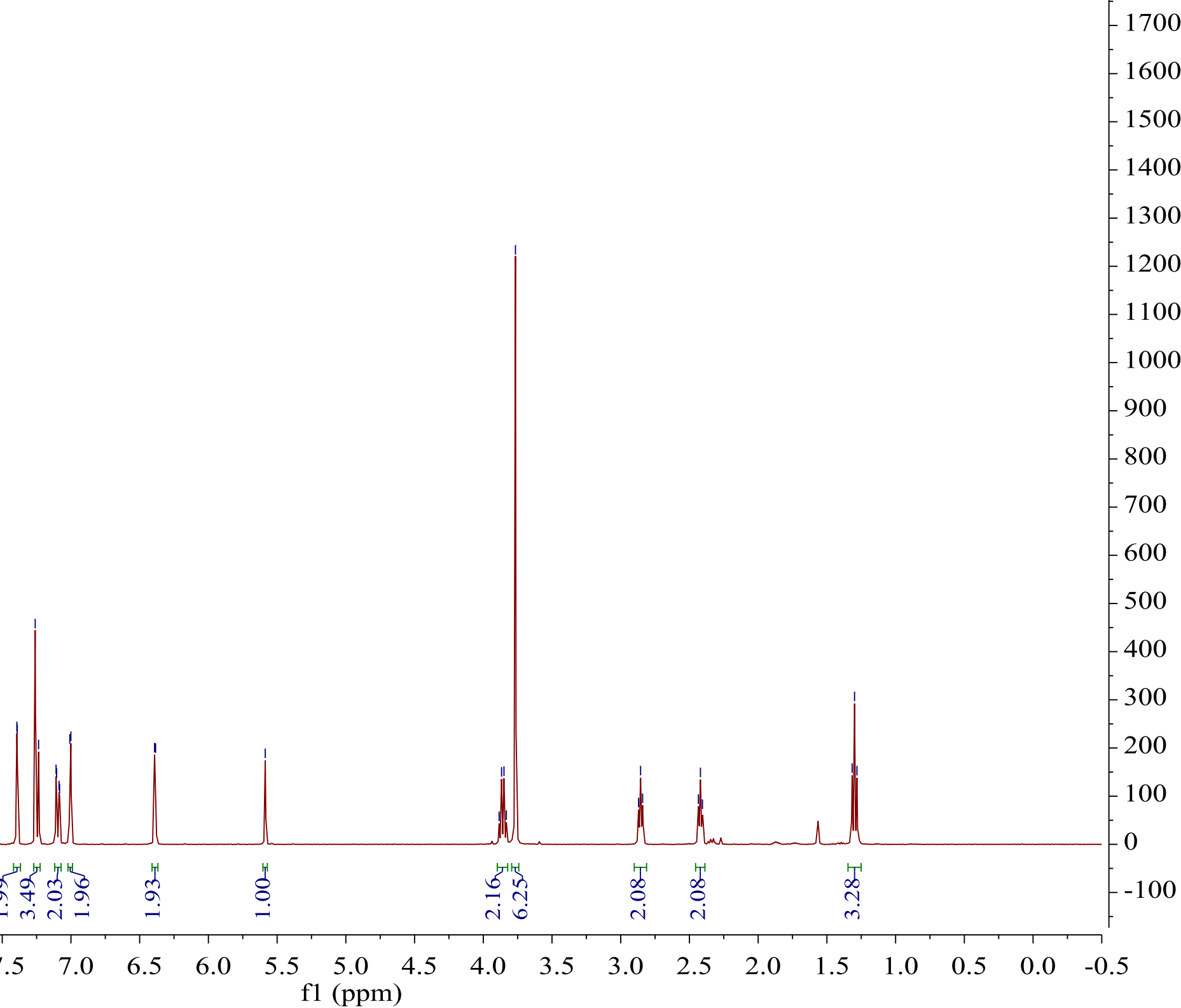


CARBON_01

${ }^{13} \mathrm{C}$ NMR spectrum of compound $3 \mathrm{~s}$
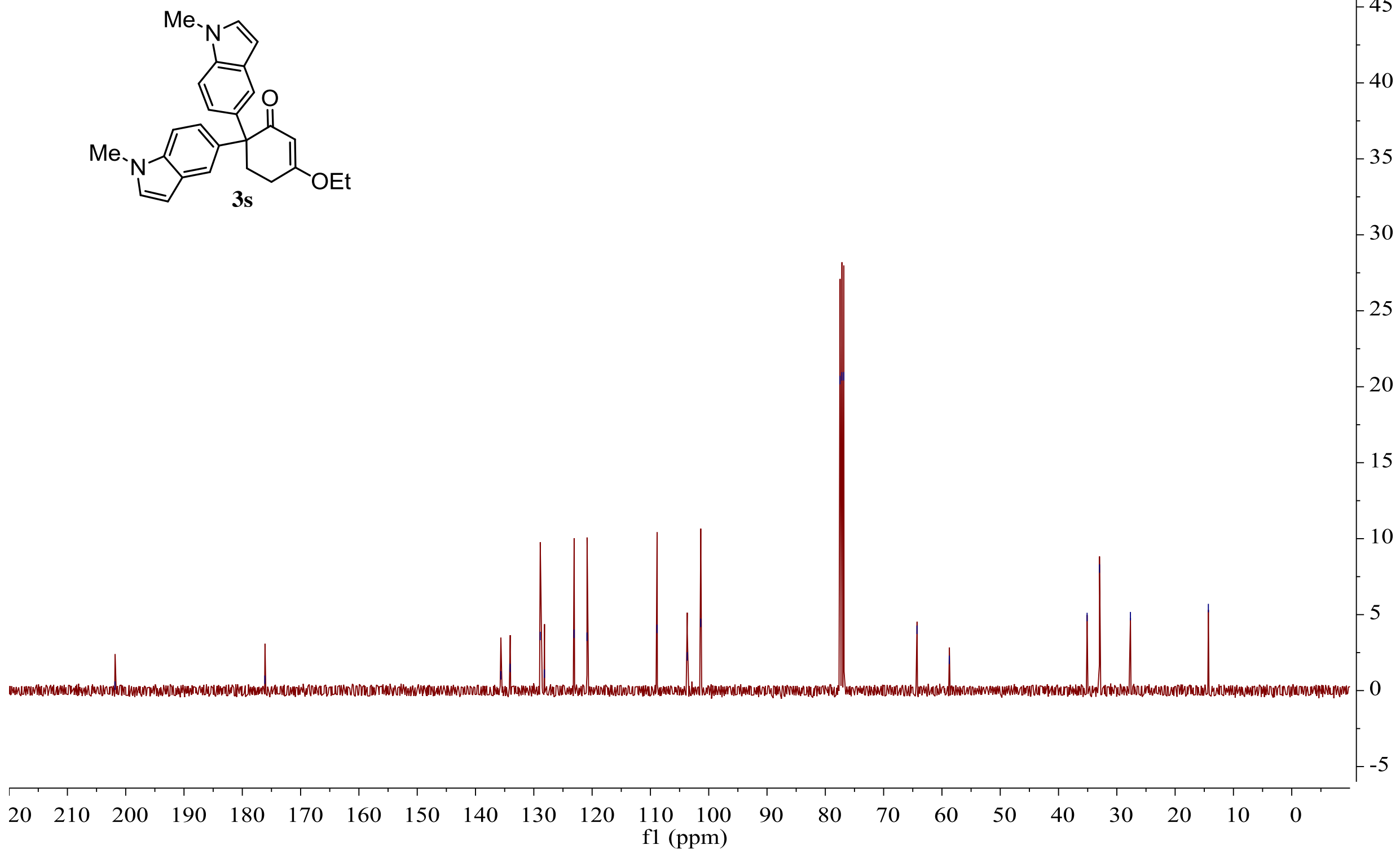
PROTON_01

${ }^{1} \mathrm{H}$ NMR spectrum of compound $3 \mathrm{t}$

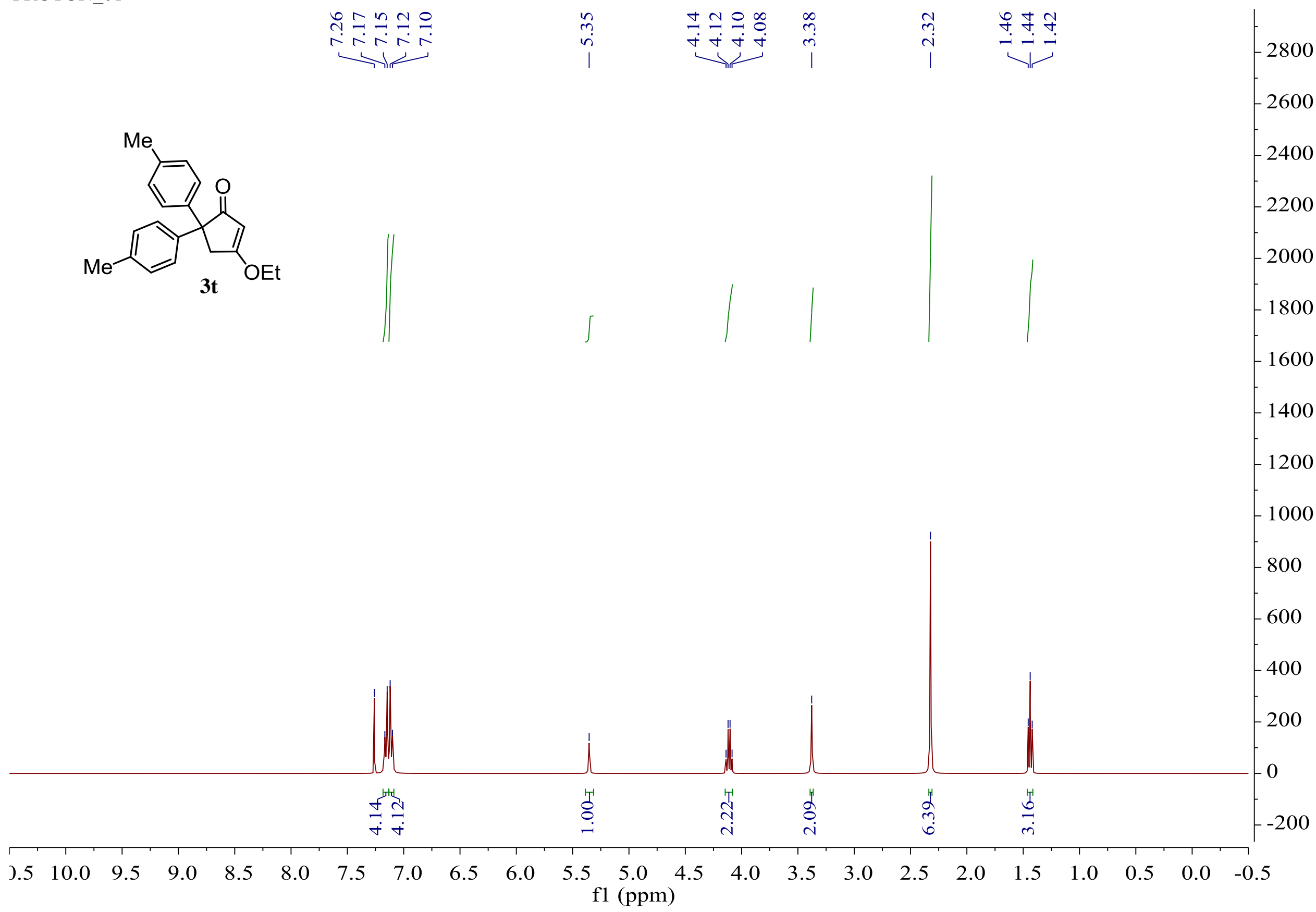




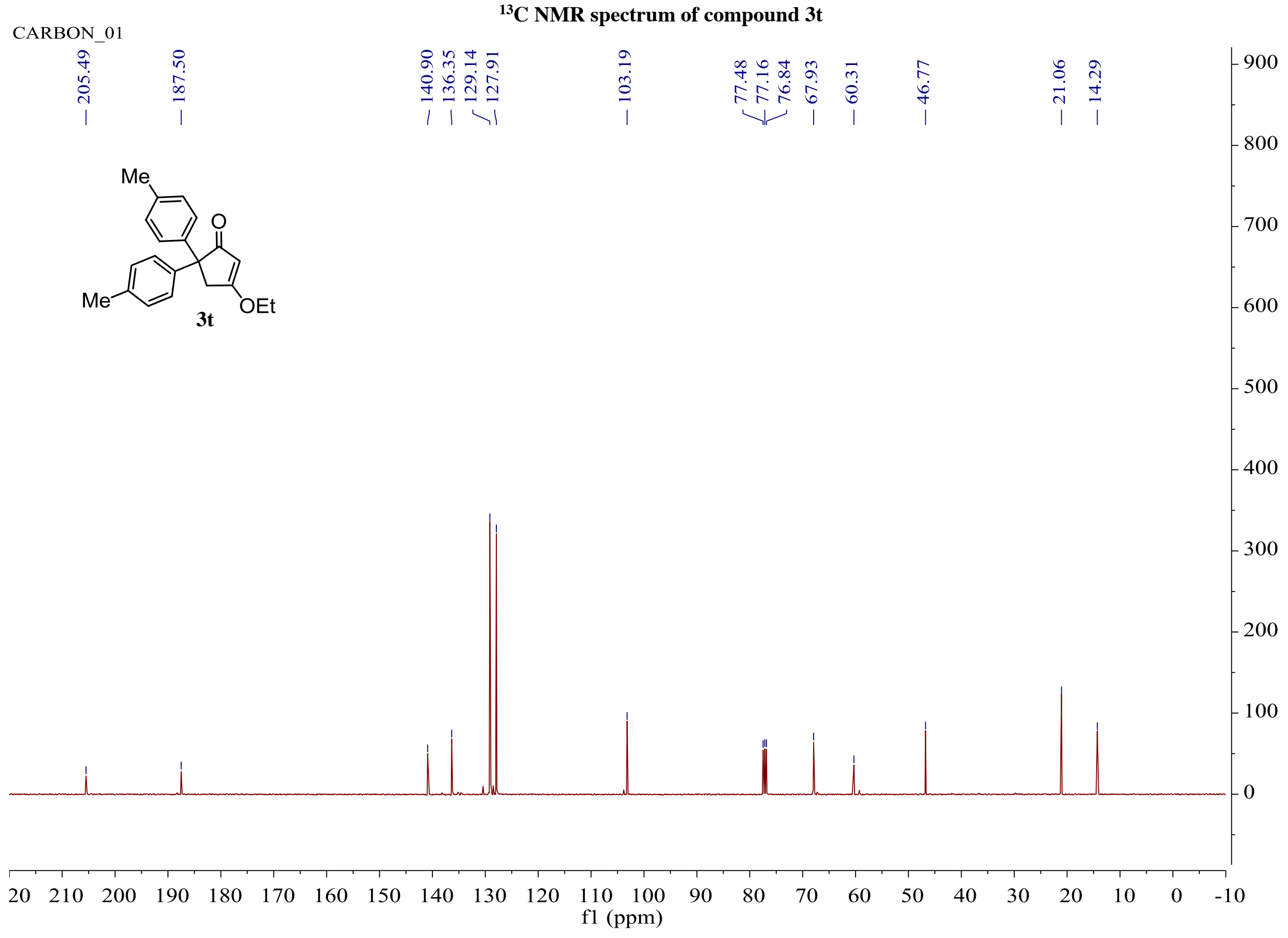


PROTON_01

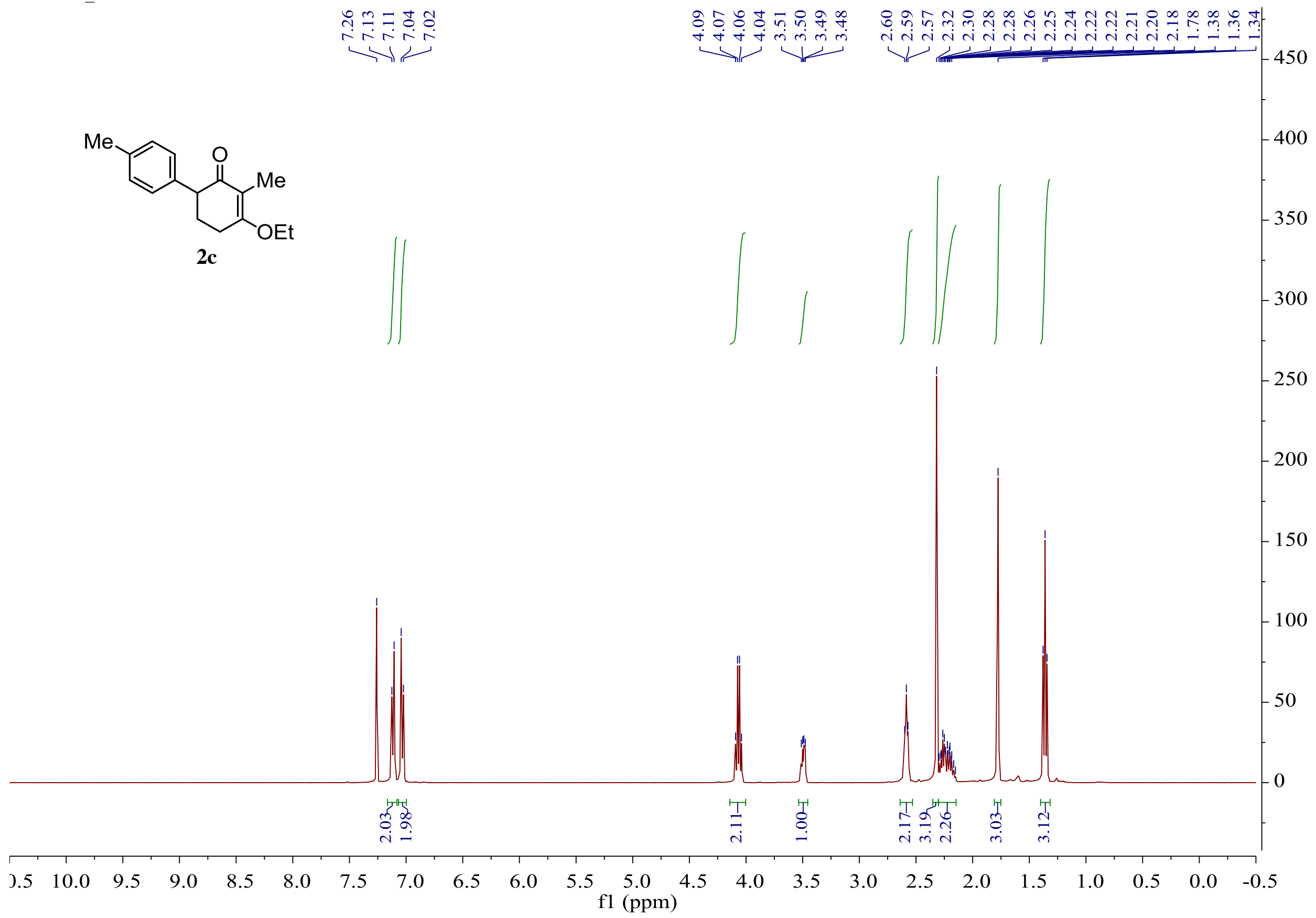

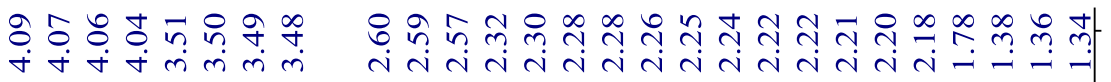

${ }^{1} \mathrm{H}$ NMR spectrum of compound $2 \mathrm{c}$ 
CARBON 01

${ }^{13} \mathrm{C}$ NMR spectrum of compound $2 \mathrm{c}$

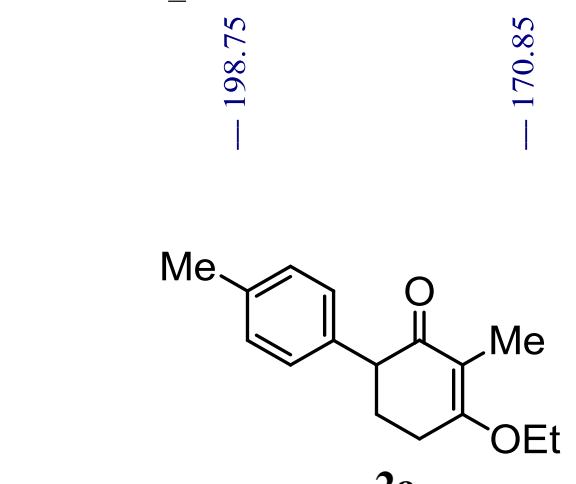

2c 
PROTON_01

${ }^{1} \mathrm{H}$ NMR spectrum of compound $4 \mathbf{a}$

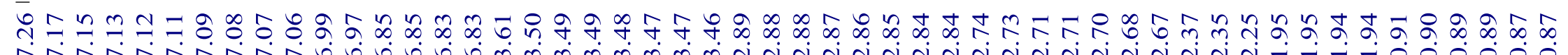<smiles>CCOC1=C(C)C(=O)C(c2ccc(C)cc2)(c2ccc(C)cc2)CC1c1ccc(C)cc1</smiles>
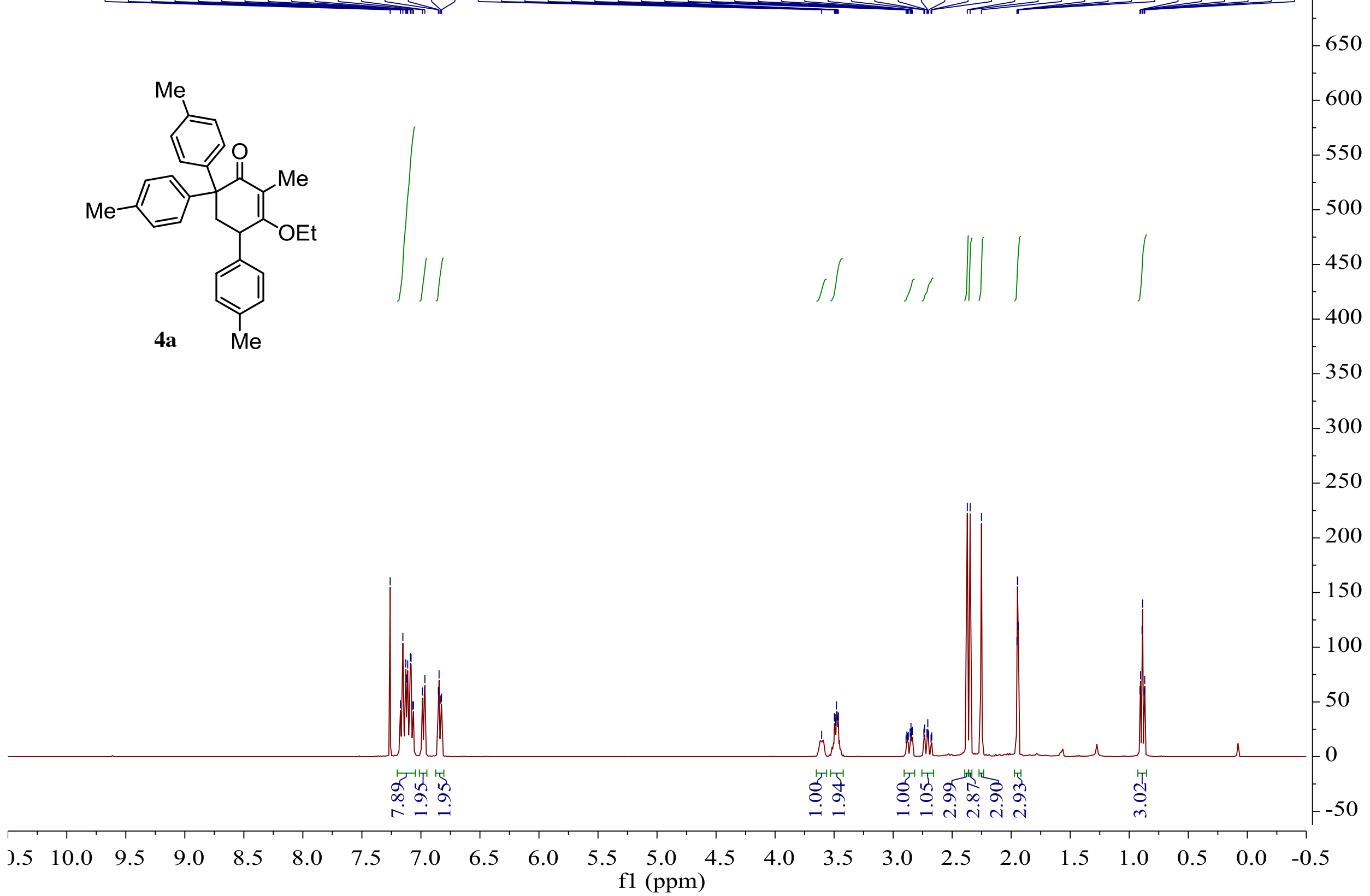
CARBON_01

${ }^{13} \mathrm{C}$ NMR spectrum of compound $4 \mathrm{a}$

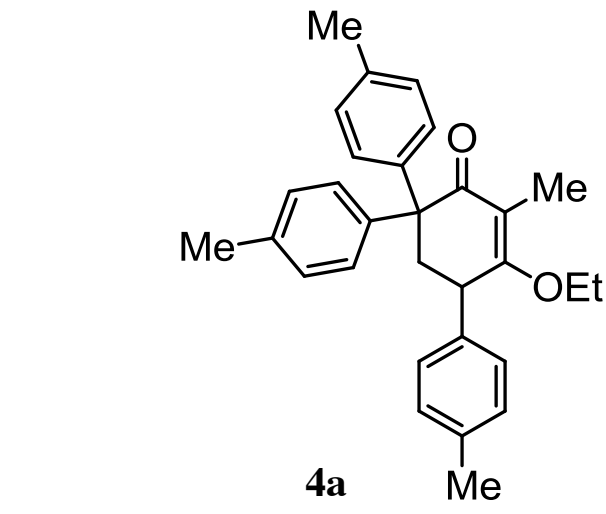

\section{OEt}

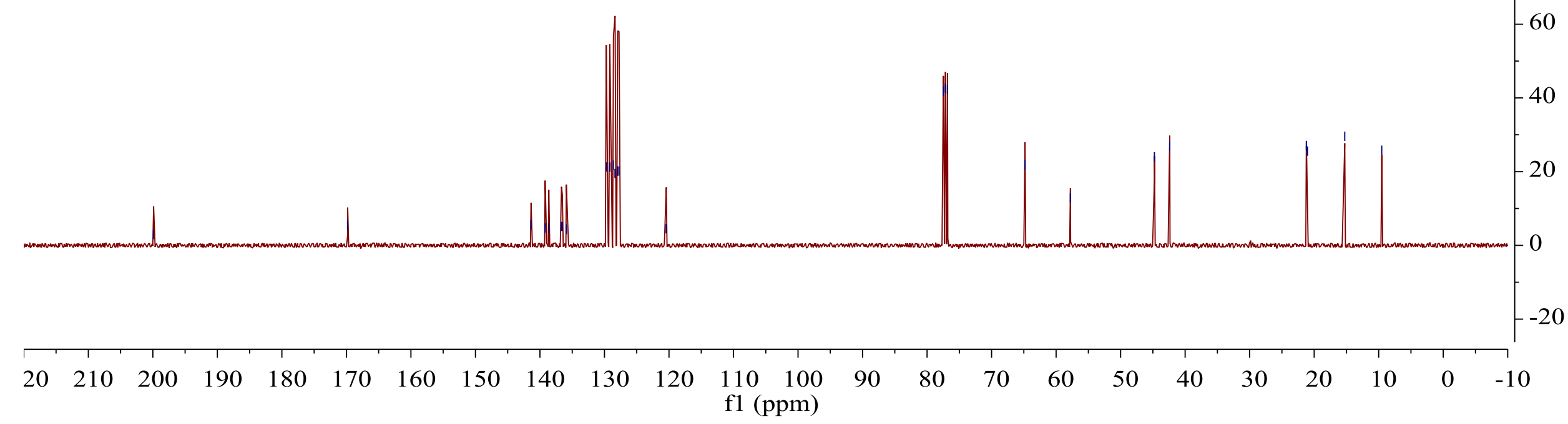


gCOSY_01

COSY spectrum of compound 4 a

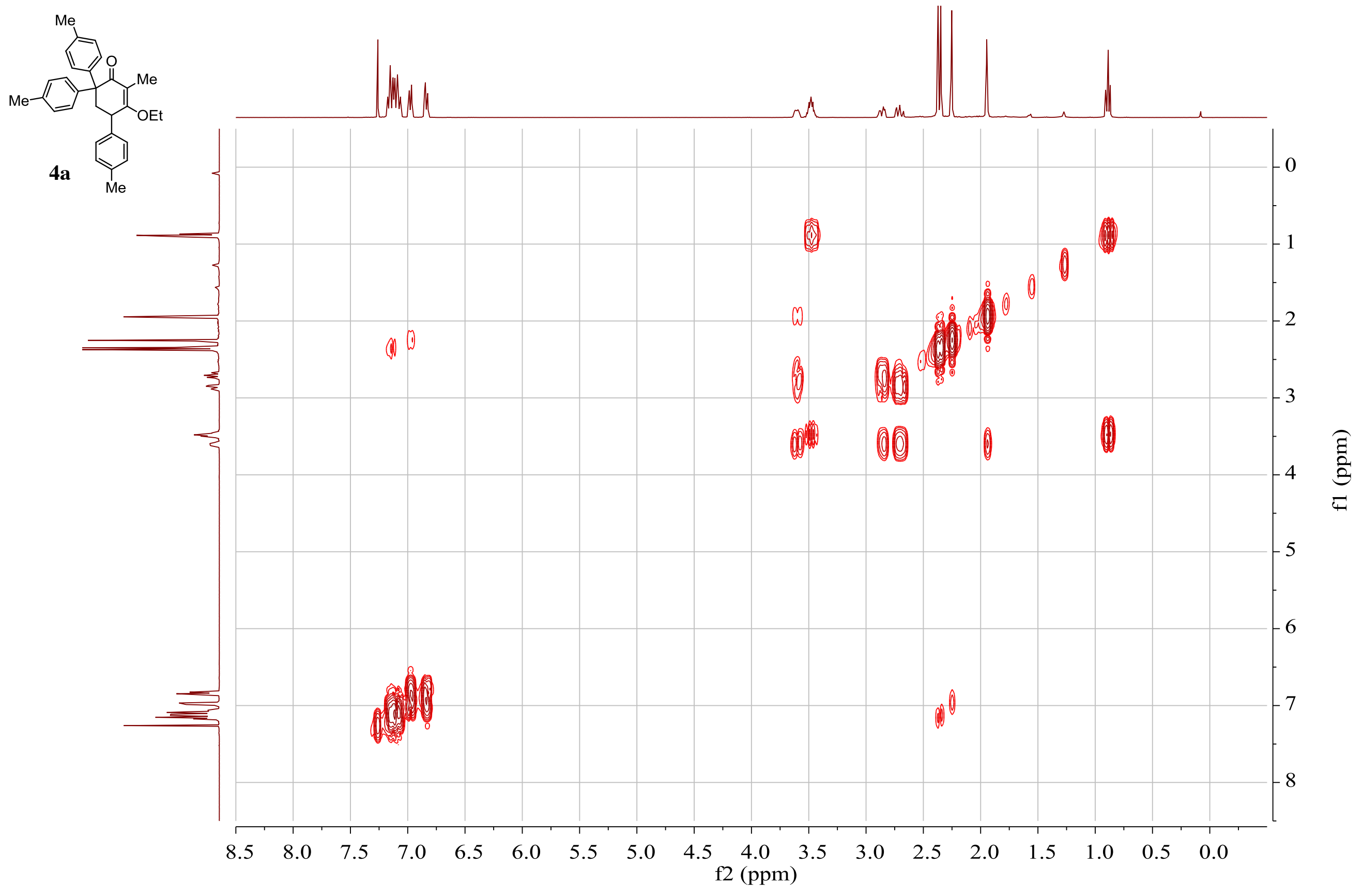


gHSQCAD_01

HSQC spectrum of compound $4 a$

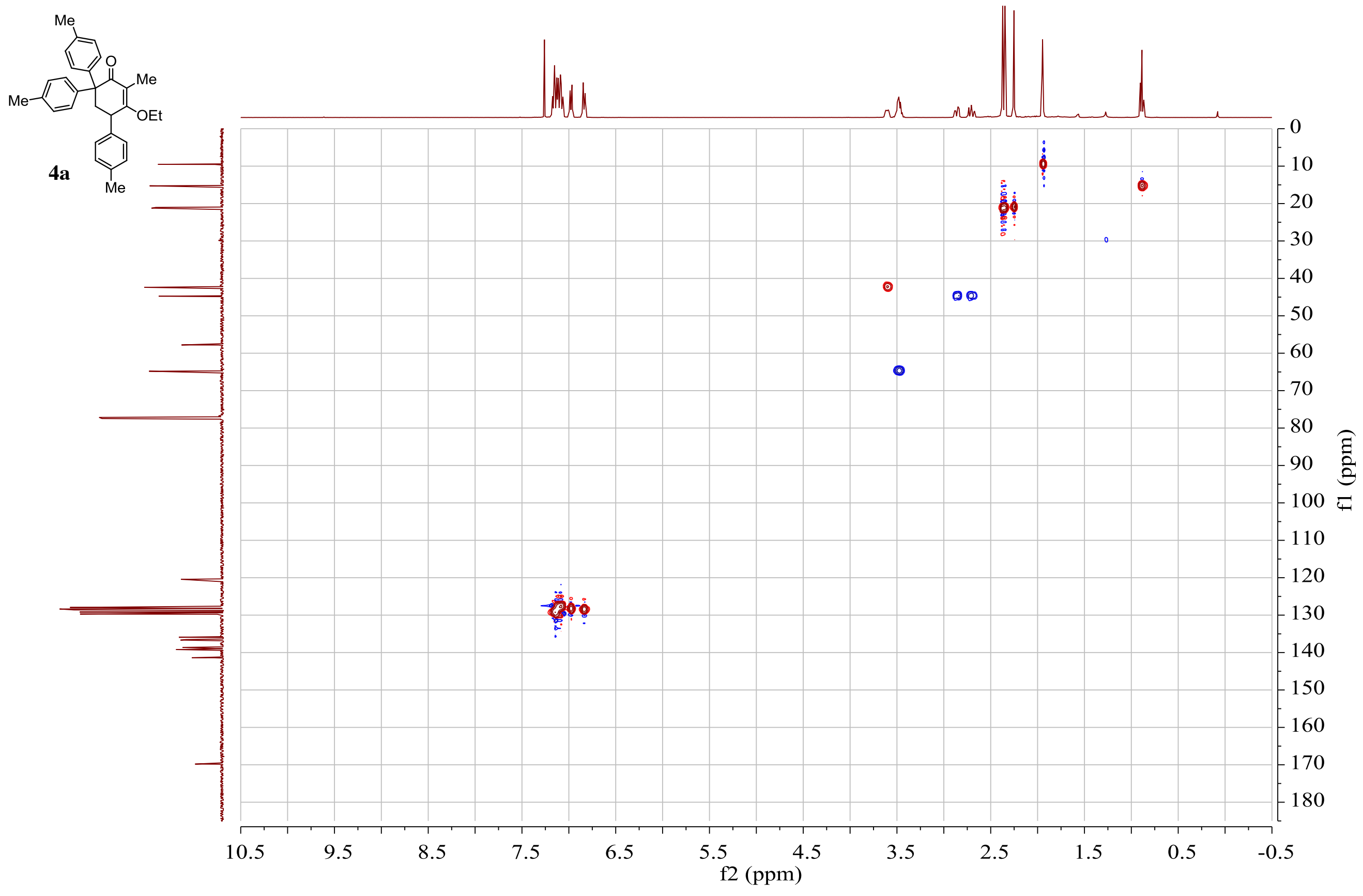




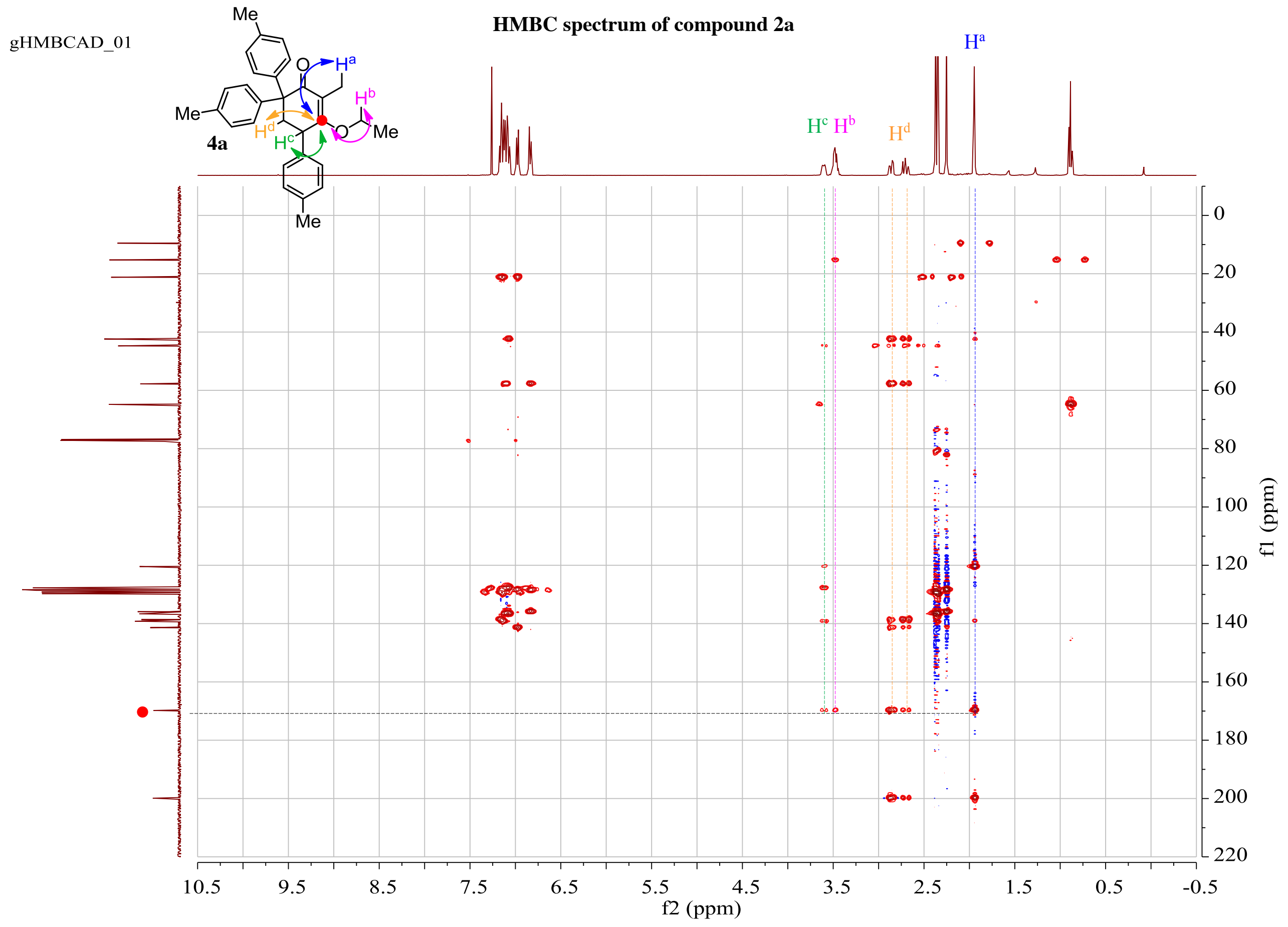




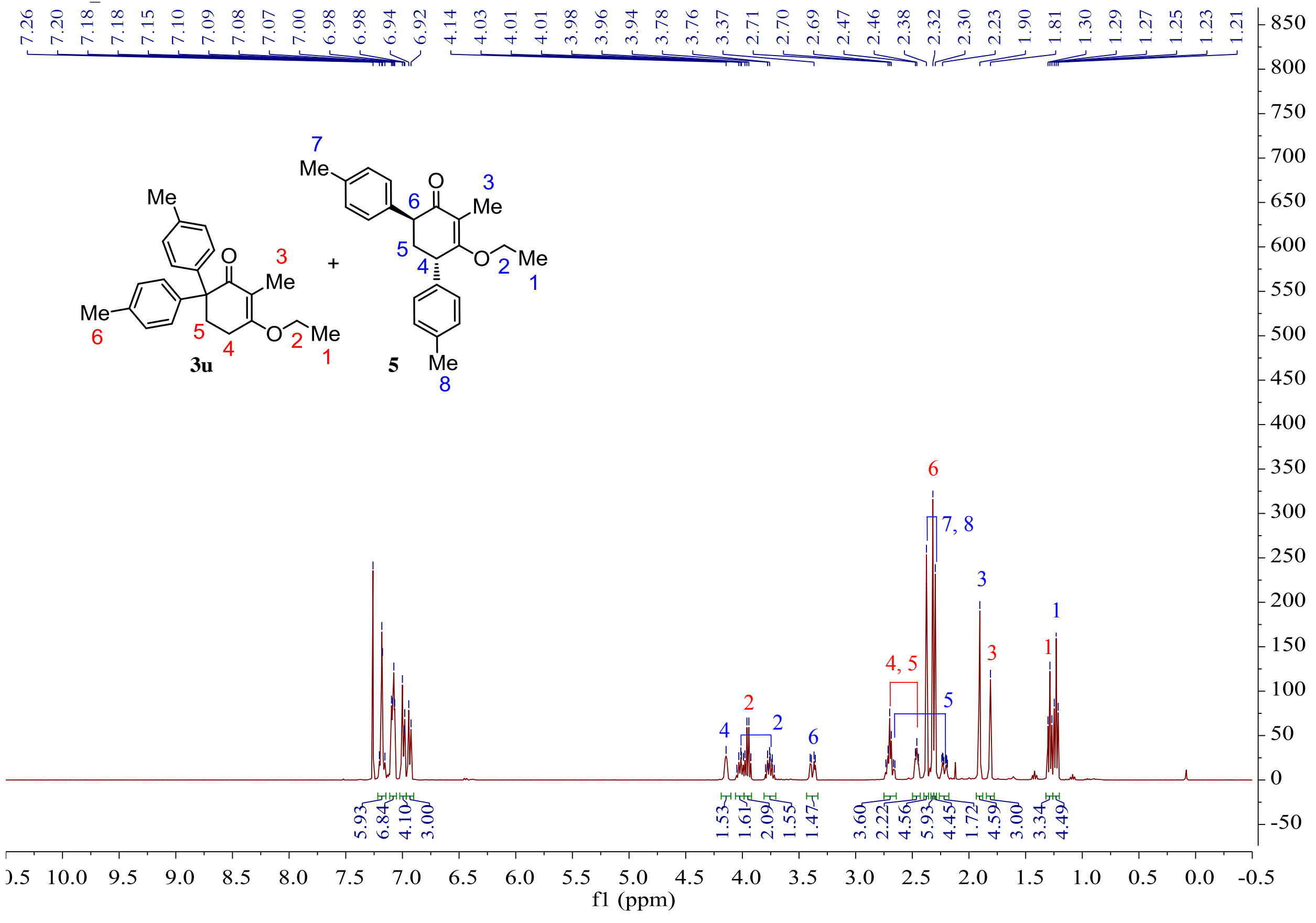

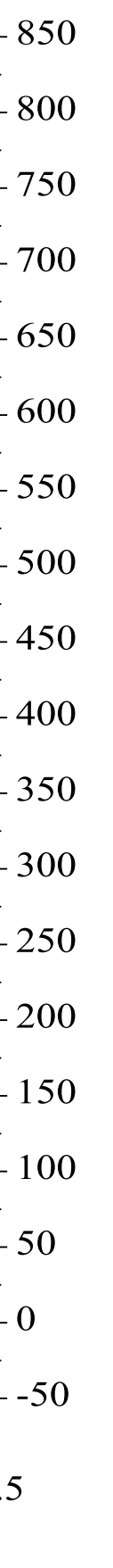


CARBON_01

${ }^{13} \mathrm{C}$ NMR spectrum of compound $3 \mathrm{u}$ and 5

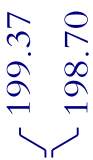

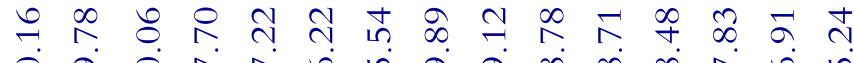

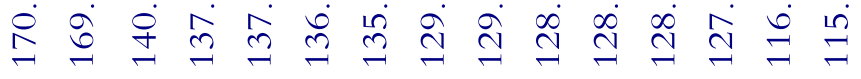

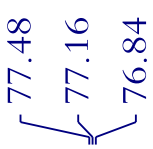

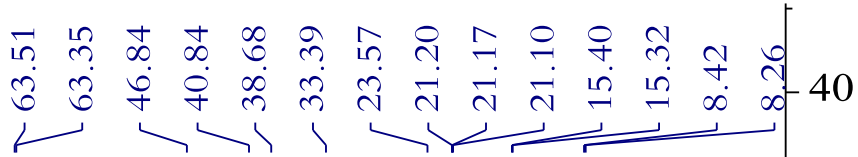

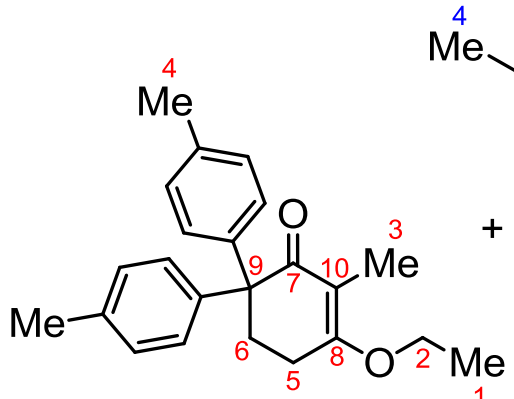

$\mathrm{Me}^{4}$
+
+
$\mathrm{Me}$

$3 \mathbf{u}$

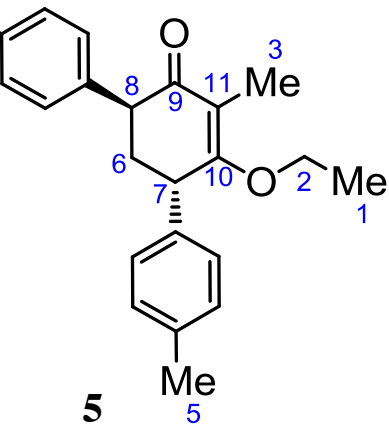

108 
gCOSY_01

COSY spectrum of compound $3 u$ and 5

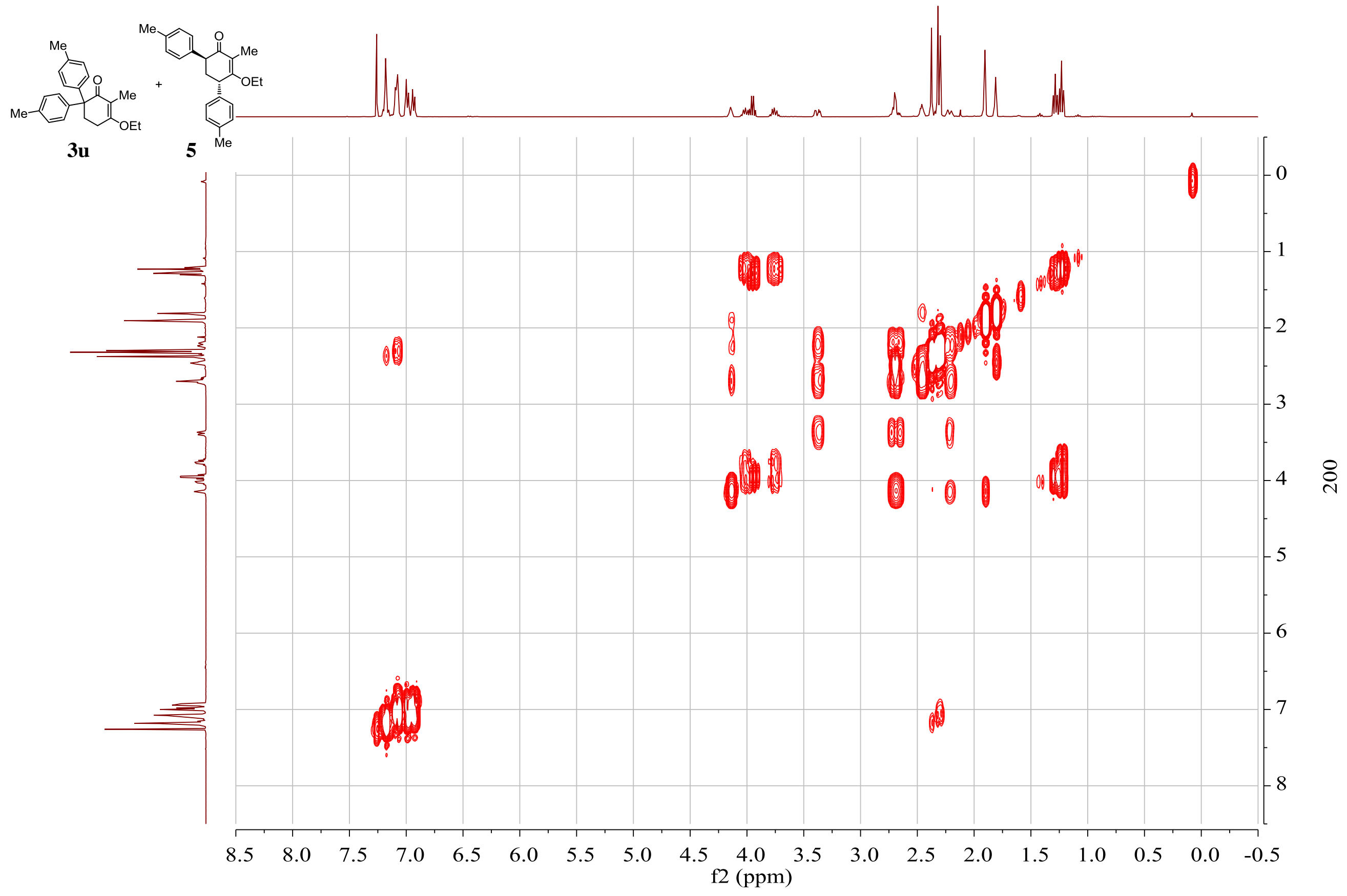


gHSQCAD_01

HSQC spectrum of compound $3 u$ and 5

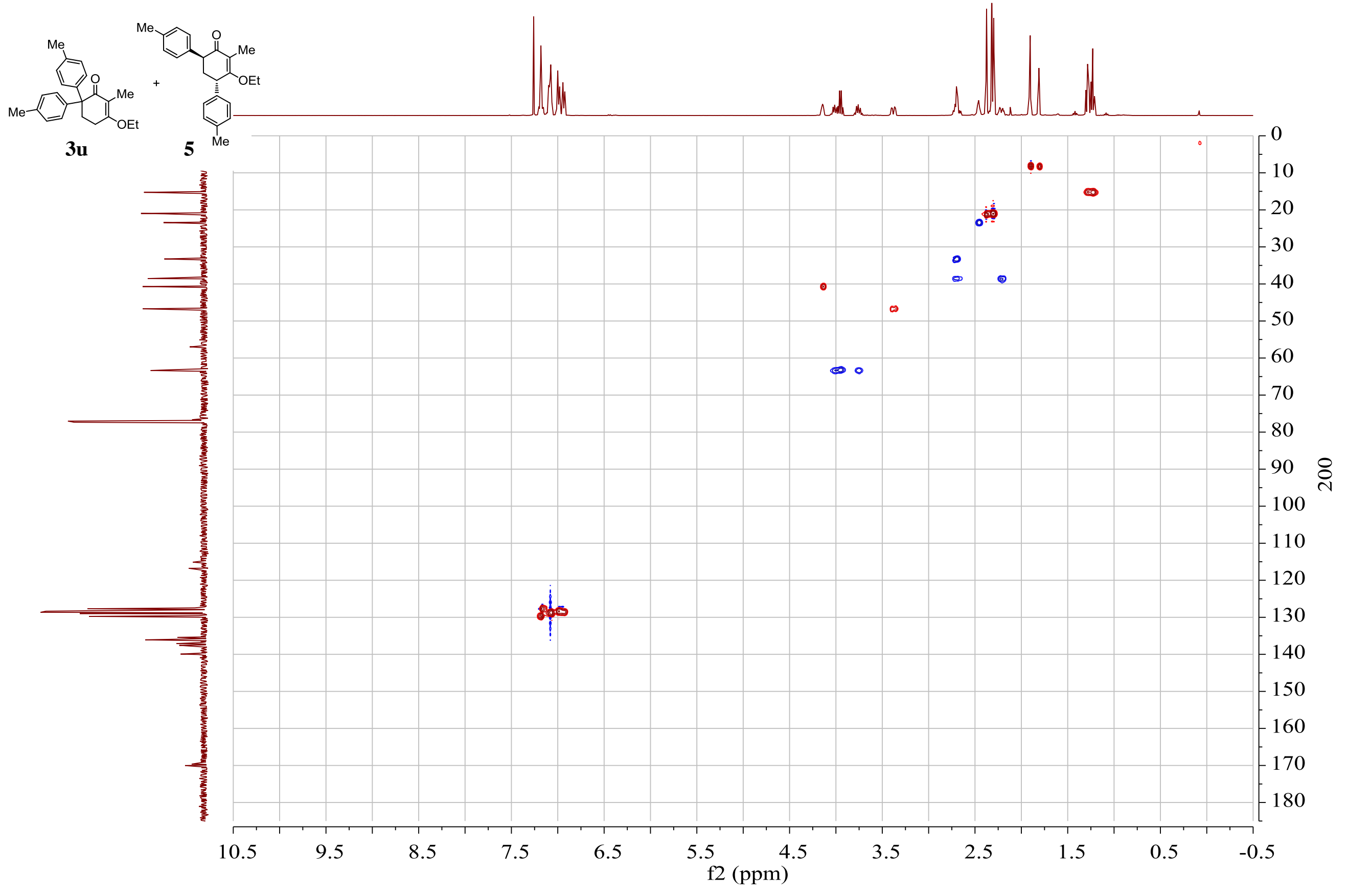


ROESYAD_01

ROESY spectrum of compound $3 u$ and 5

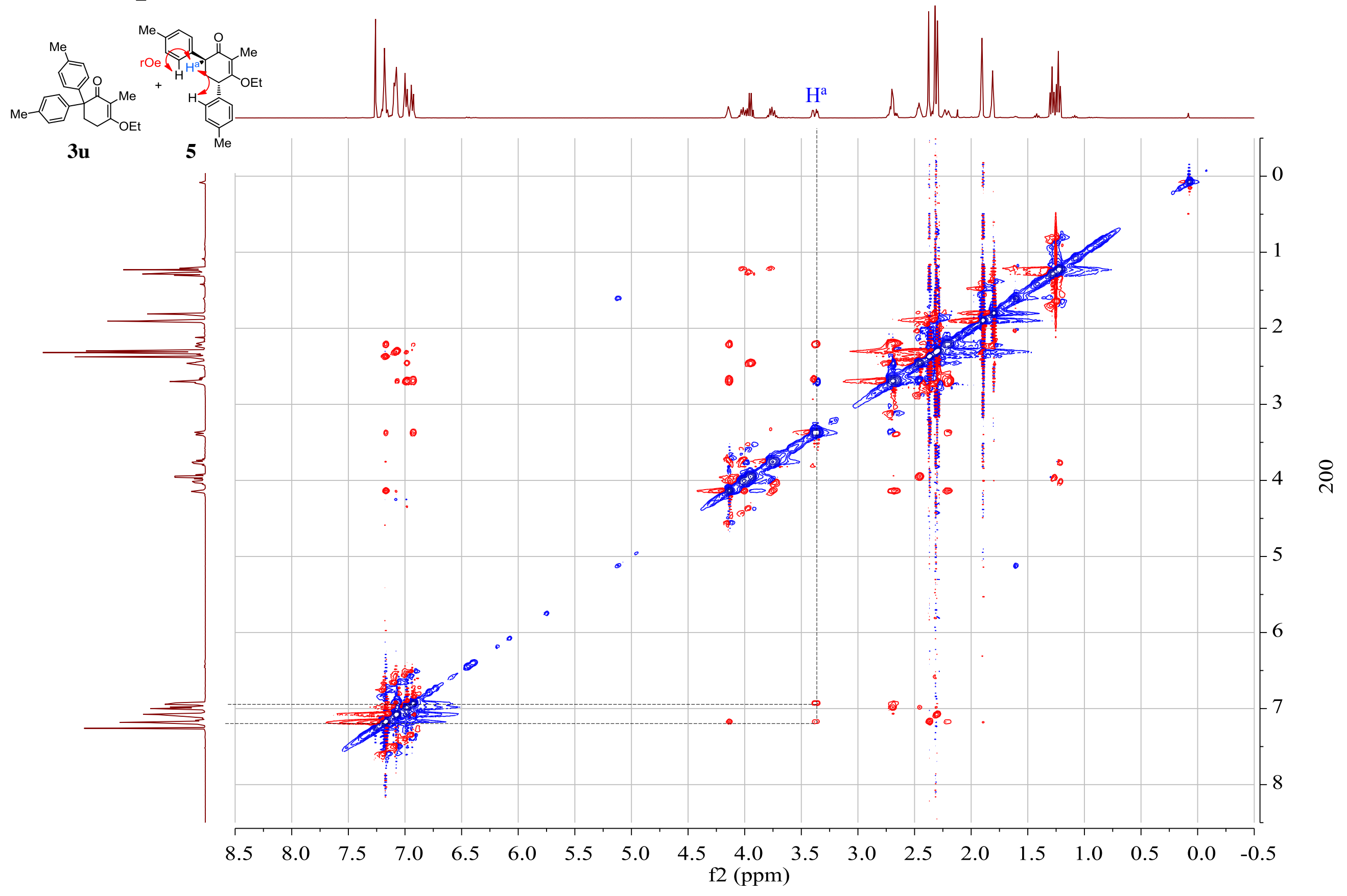




\section{${ }^{1} \mathrm{H}$ NMR spectrum of compound $4 \mathrm{~b}$}

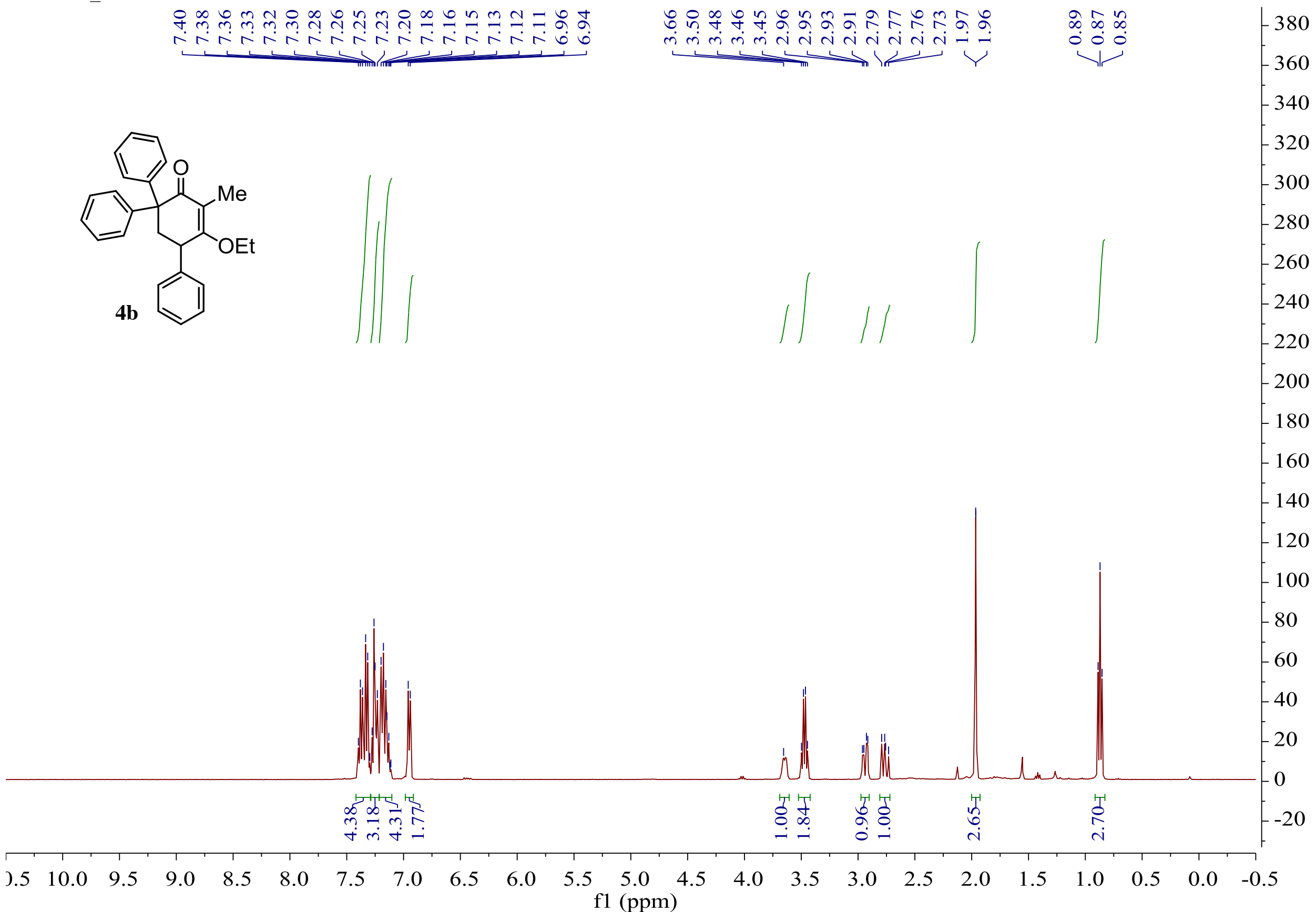


CARBON 01

年

\begin{tabular}{l}
\multirow{0}{0}{} \\
$\stackrel{1}{0}$
\end{tabular}

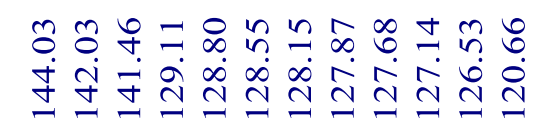

i

${ }^{13} \mathrm{C}$ NMR spectrum of compound $4 \mathrm{~b}$

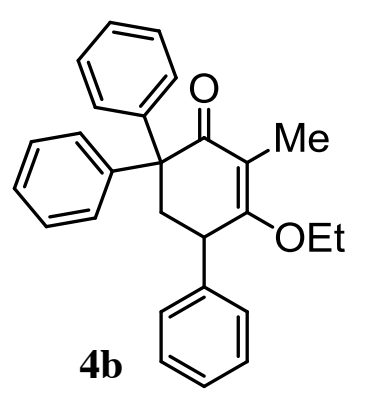

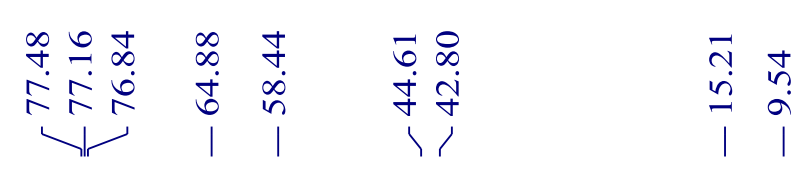

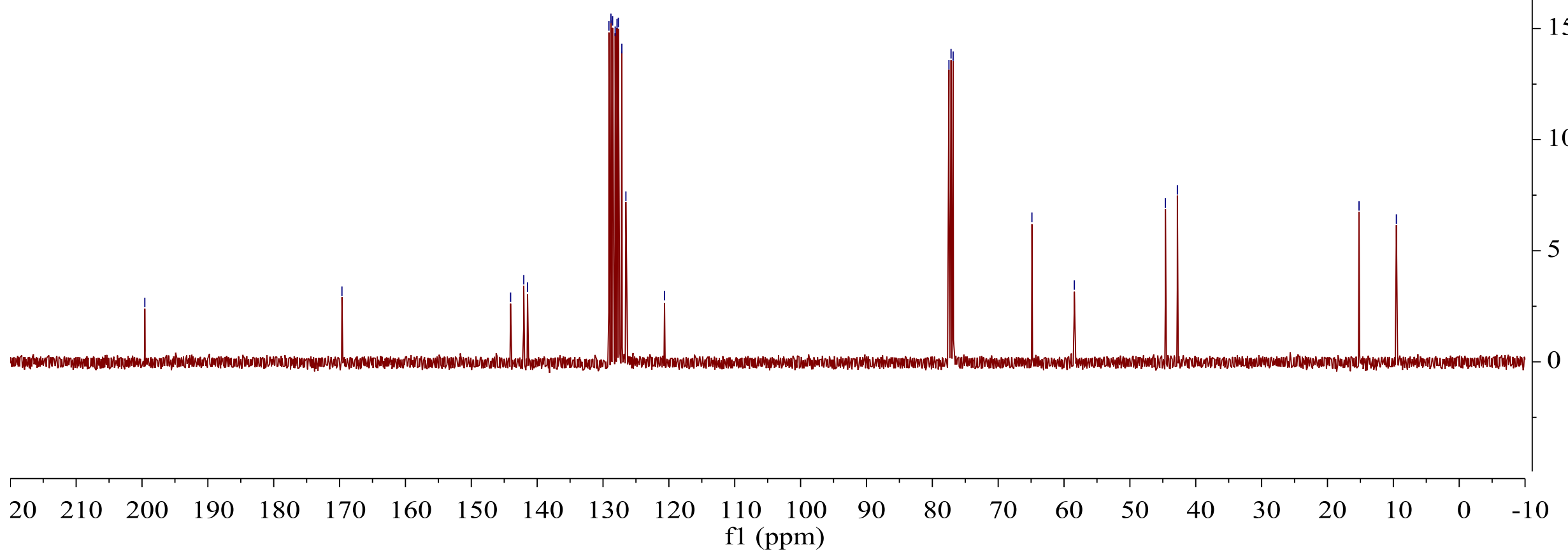




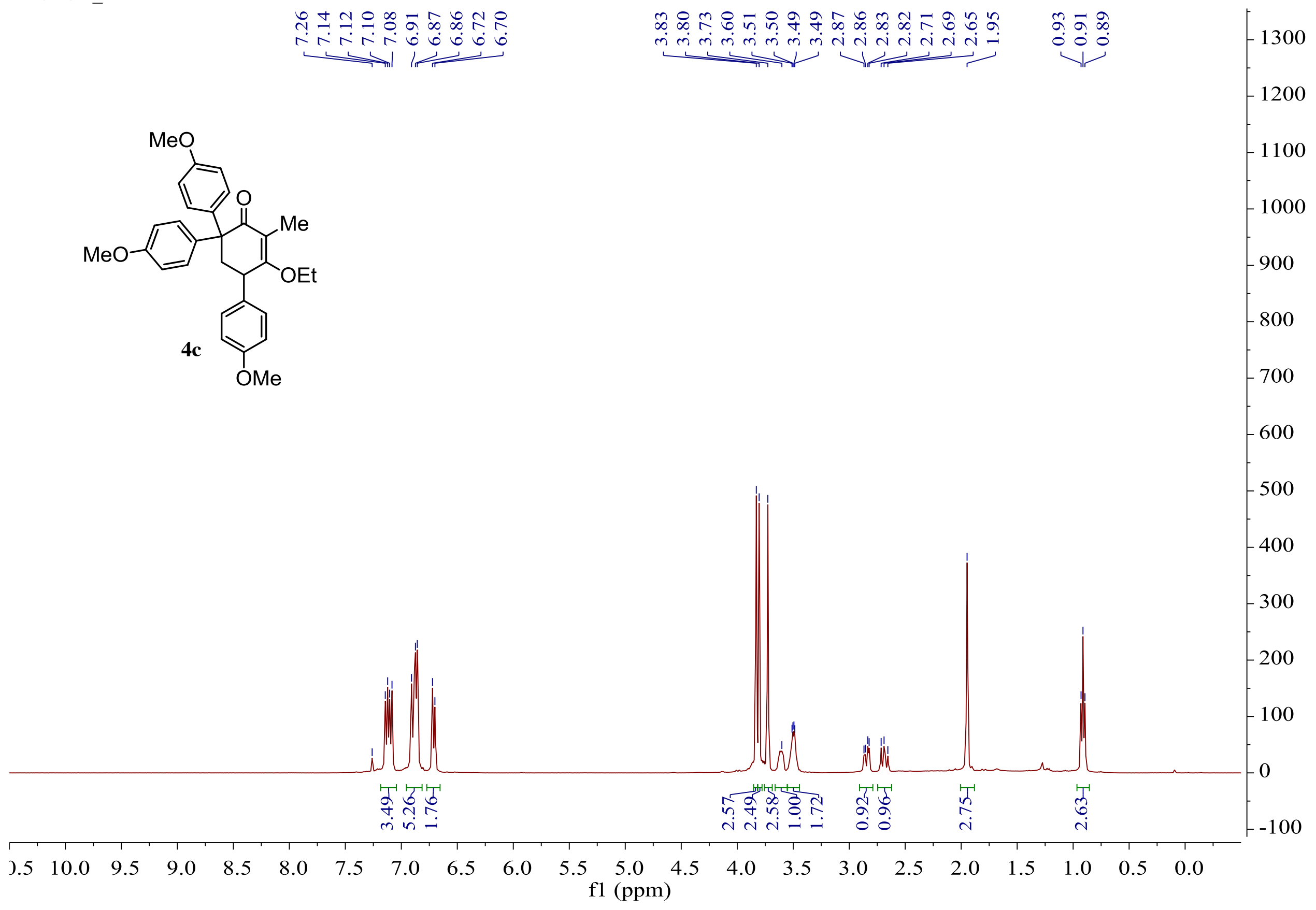




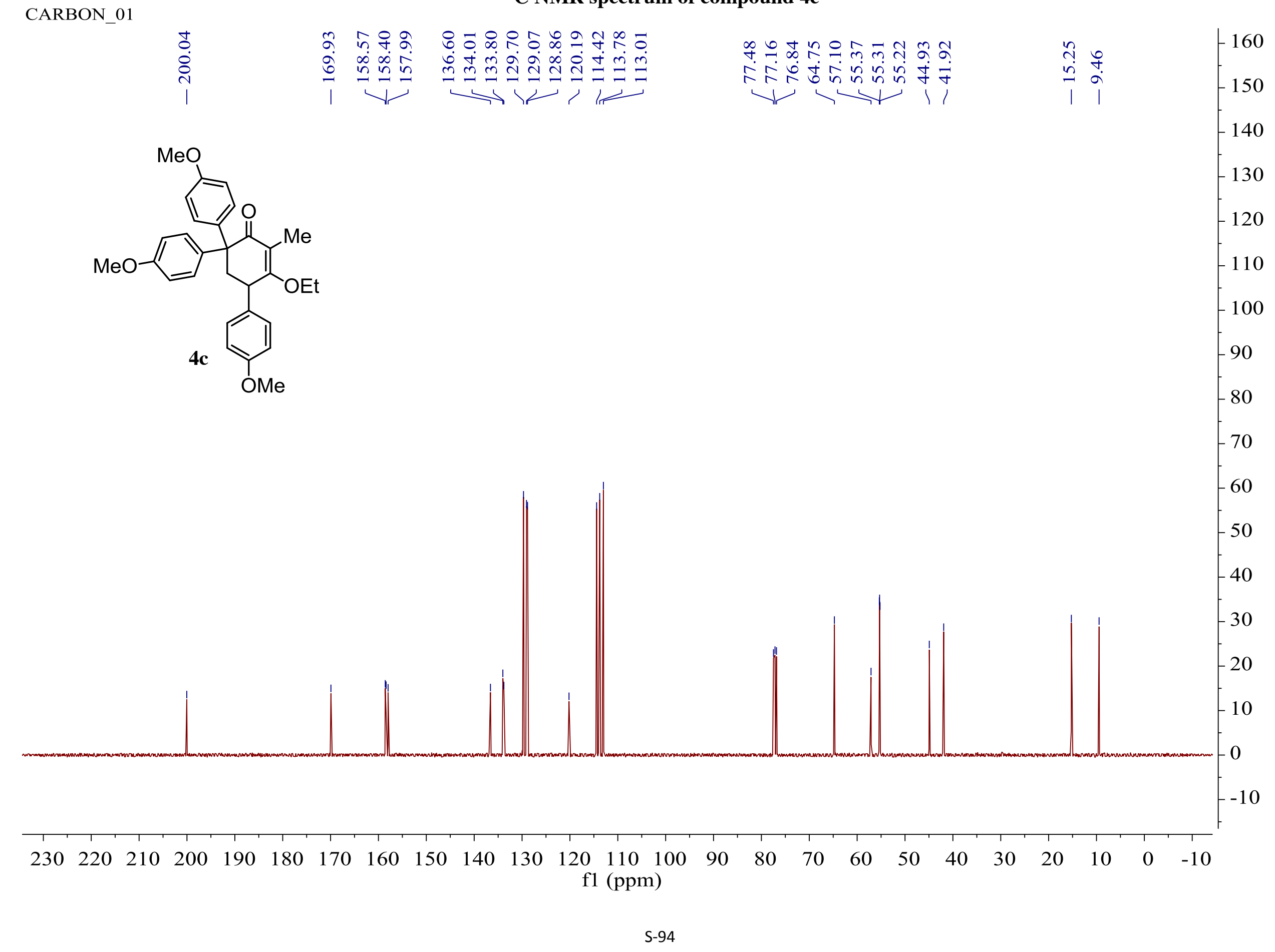

${ }^{13} \mathrm{C}$ NMR spectrum of compound $4 \mathrm{c}$ 

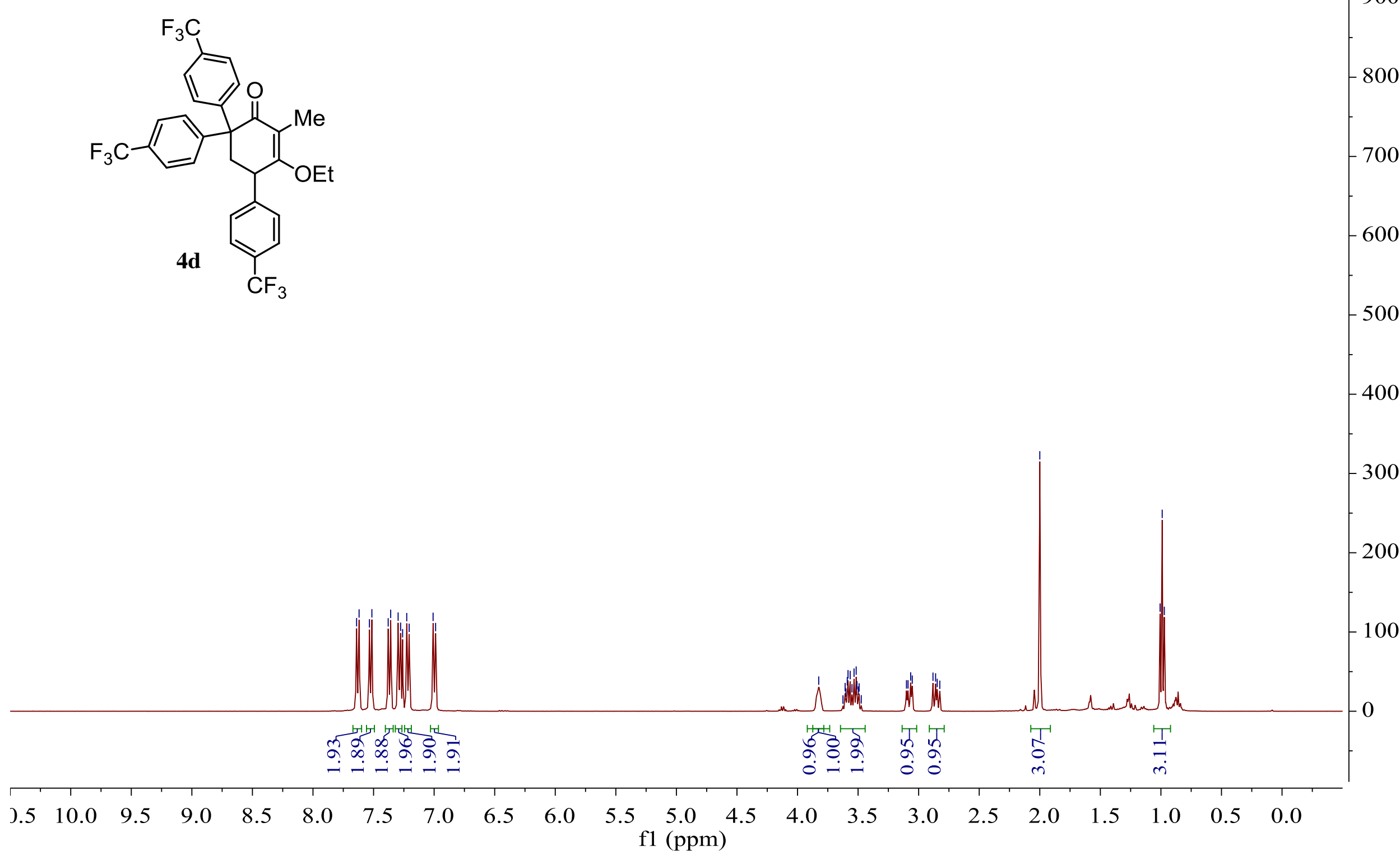


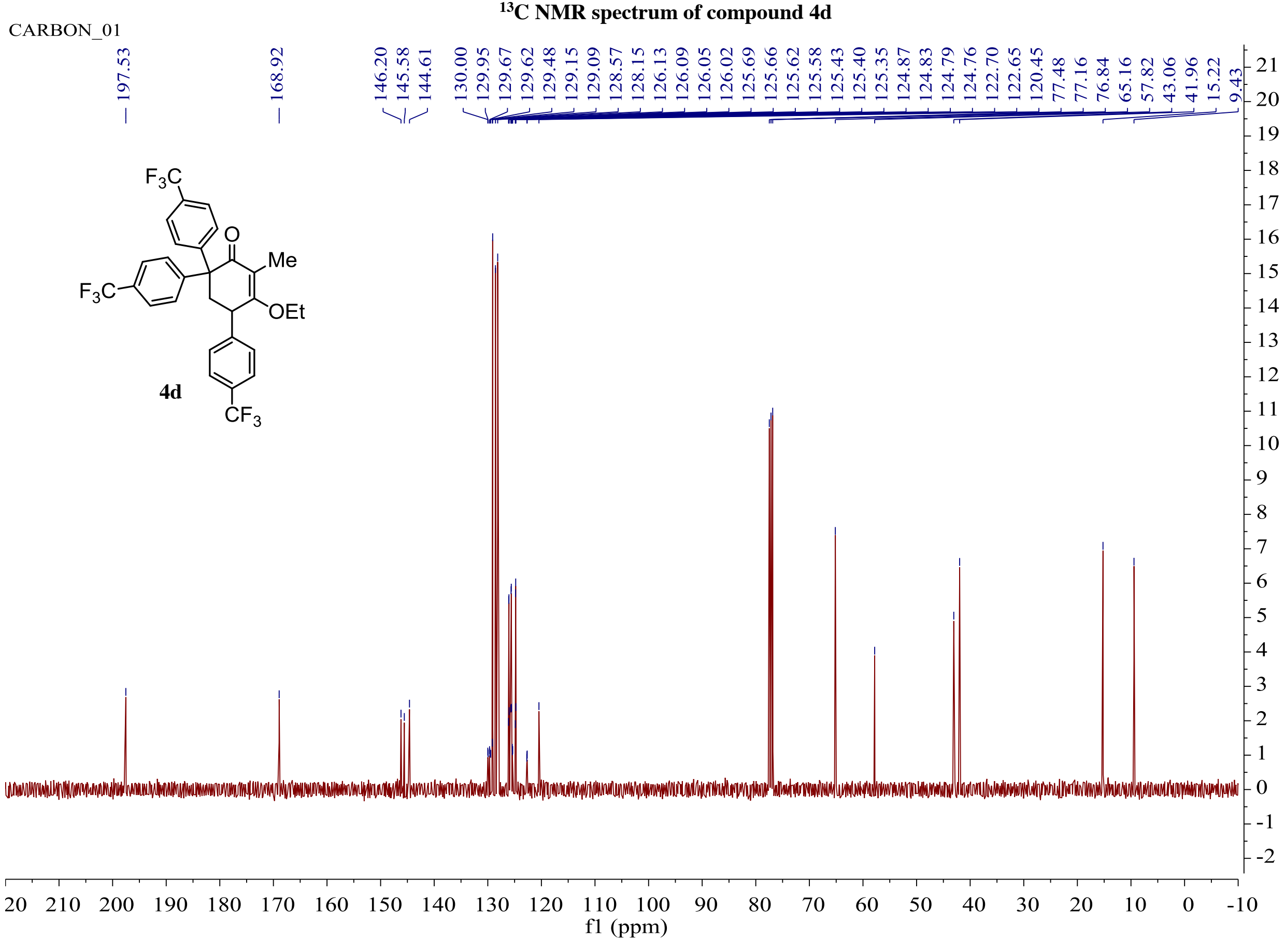




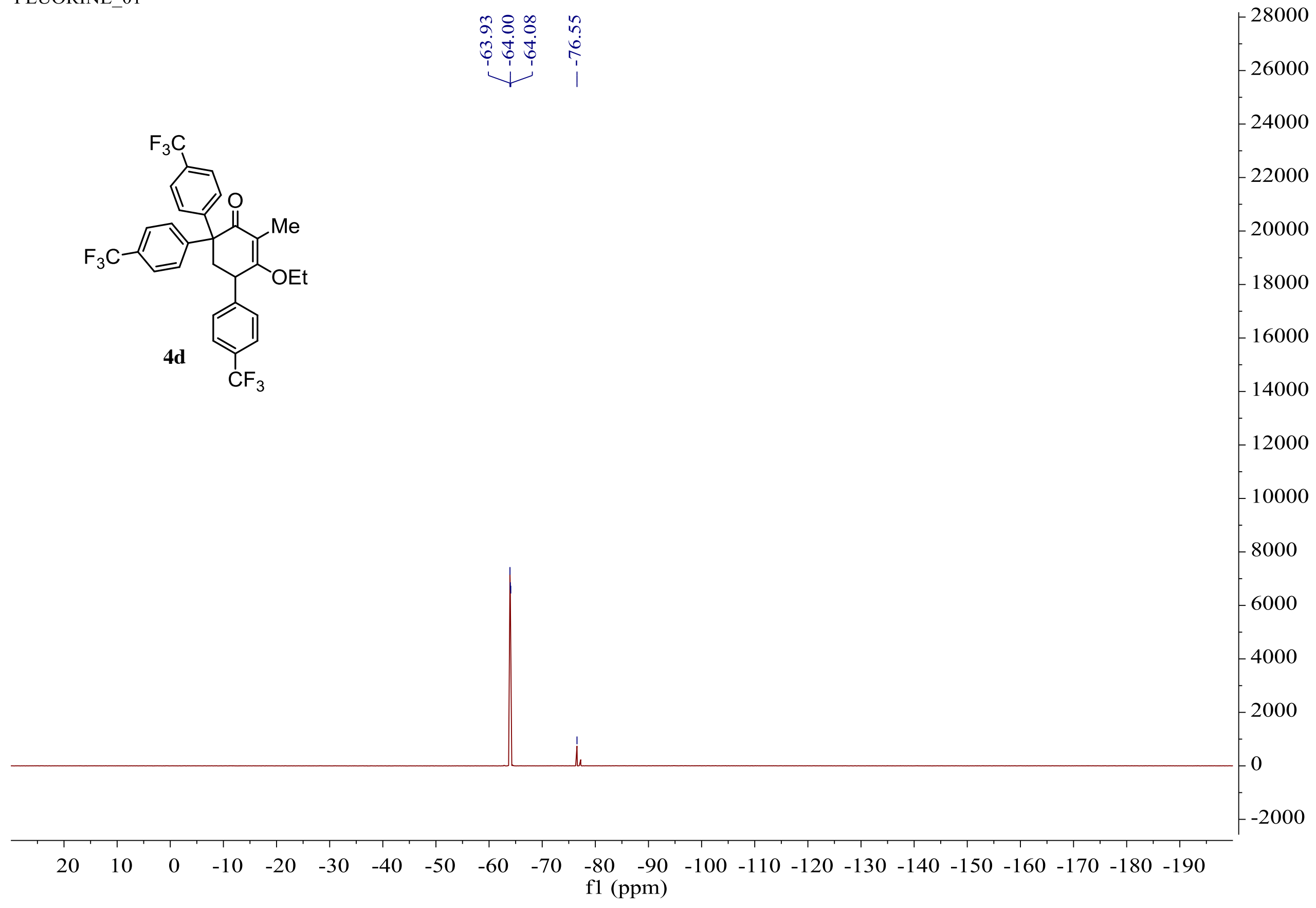




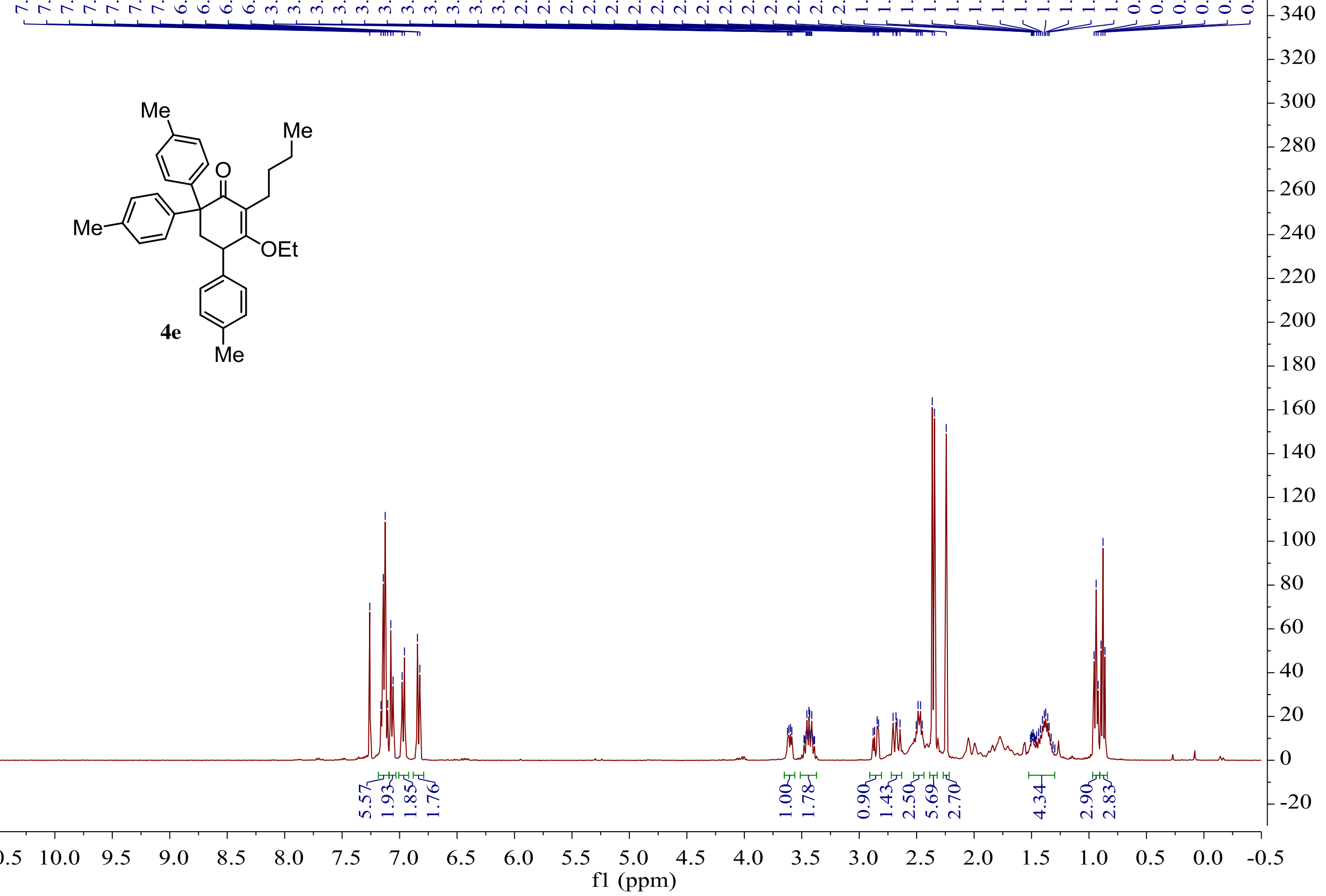


CARBON_01

${ }^{13} \mathrm{C}$ NMR spectrum of compound $4 \mathrm{e}$
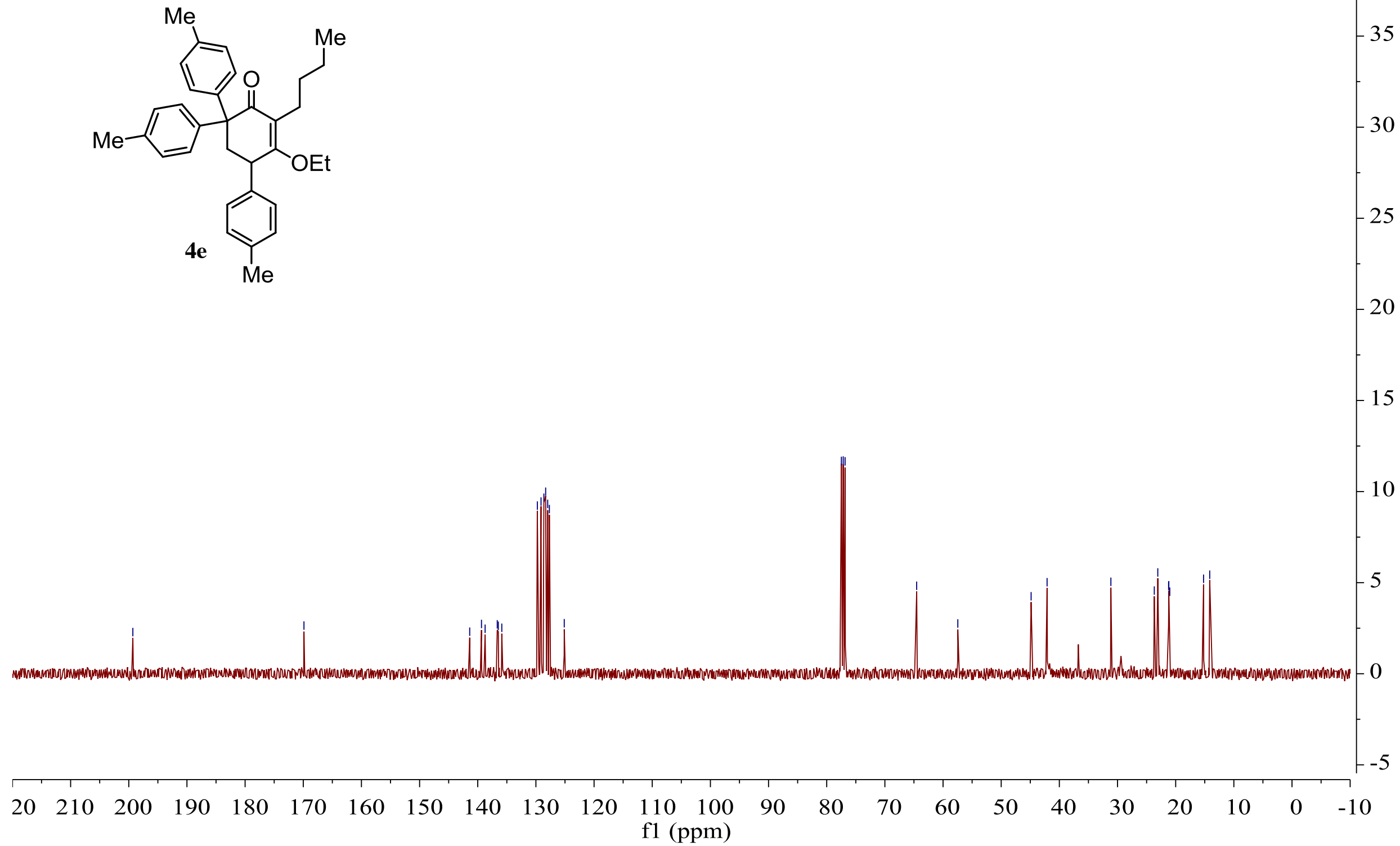


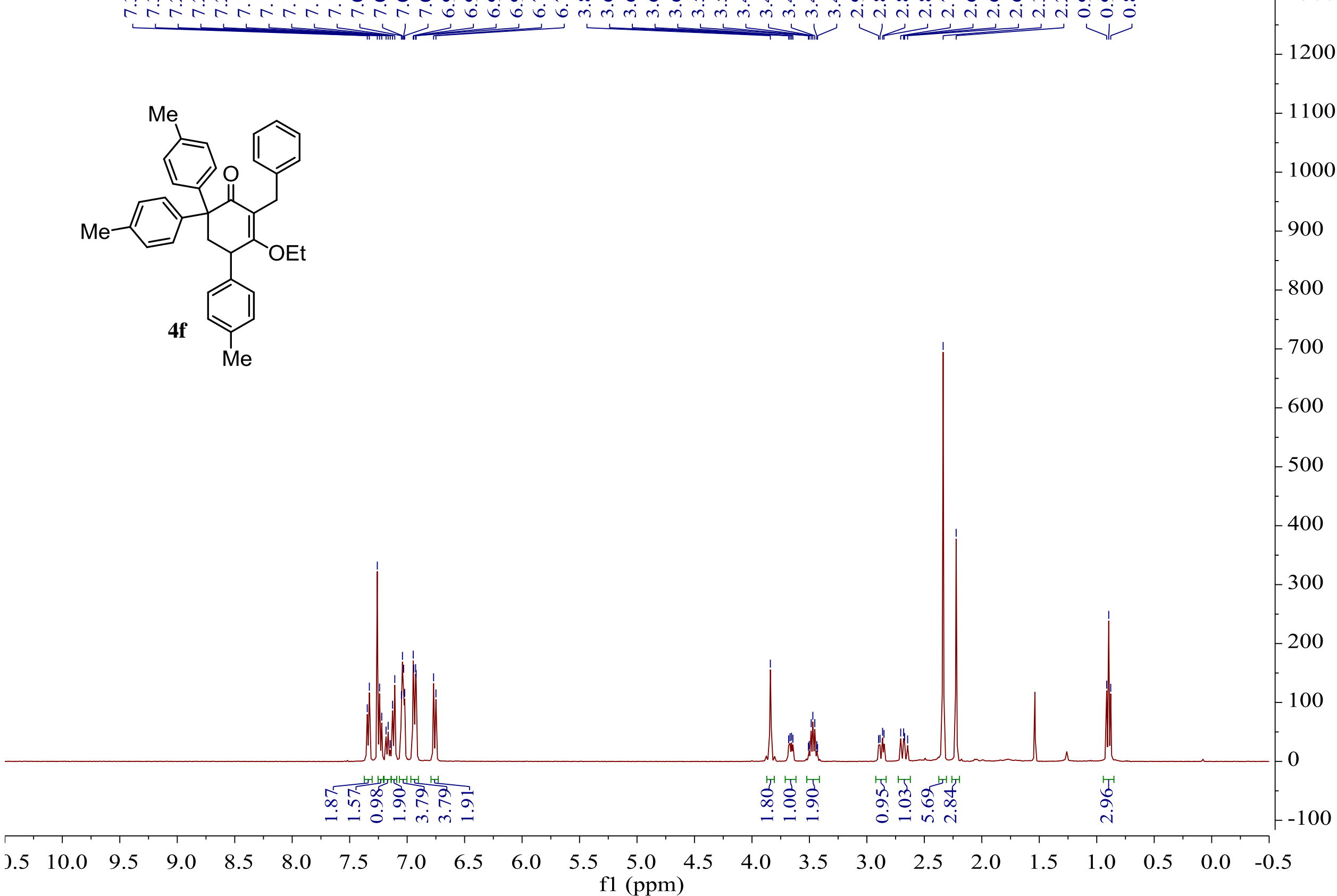


CARBON 01

${ }^{13} \mathrm{C}$ NMR spectrum of compound $4 \mathrm{f}$

$\infty$
$\infty$
$\infty$
0
1

궁

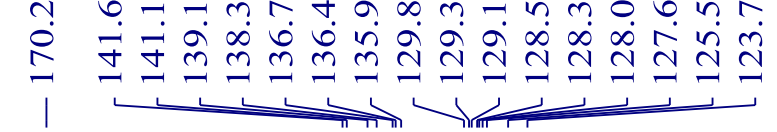

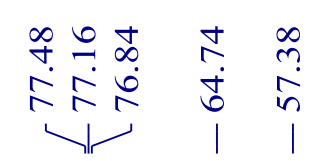

$\underset{\substack{1 \\ \stackrel{4}{+}}}{\stackrel{4}{d}}$

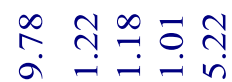

จेंतें

$\mathrm{Me}$<smiles>CCOC1=C(Cc2ccccc2)C(=O)C(c2ccc(C)cc2)(c2ccc(C)cc2)CC1c1ccc(C)cc1</smiles>

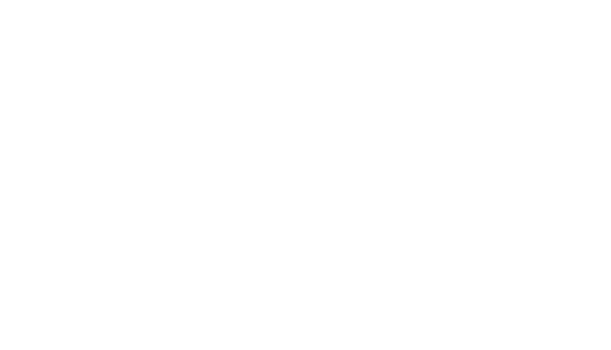

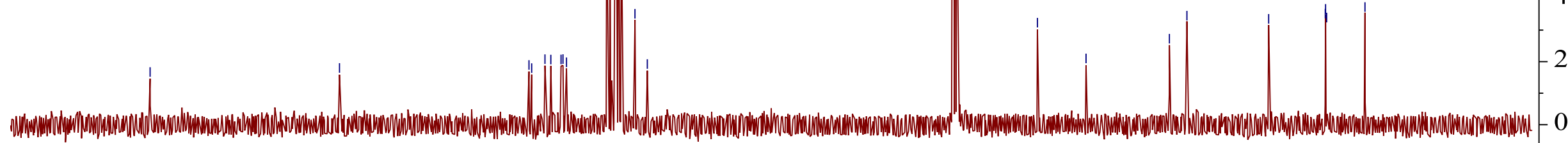

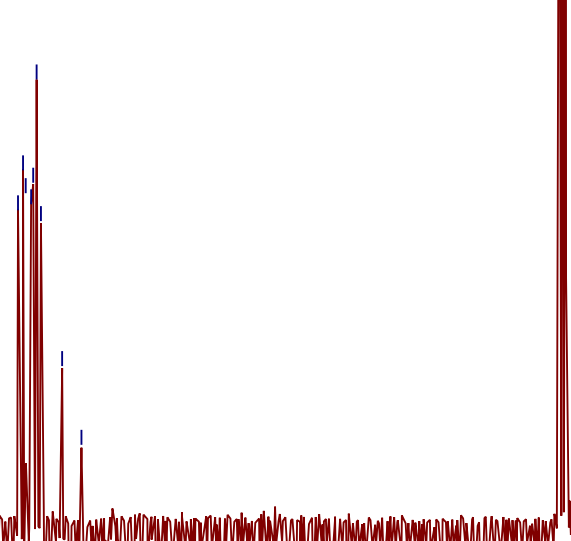

$\begin{array}{lllllllllllll}20 & 210 & 200 & 190 & 180 & 170 & 160 & 150 & 140 & 130 & 120 & 110 & 100\end{array}$ f1 (ppm) 


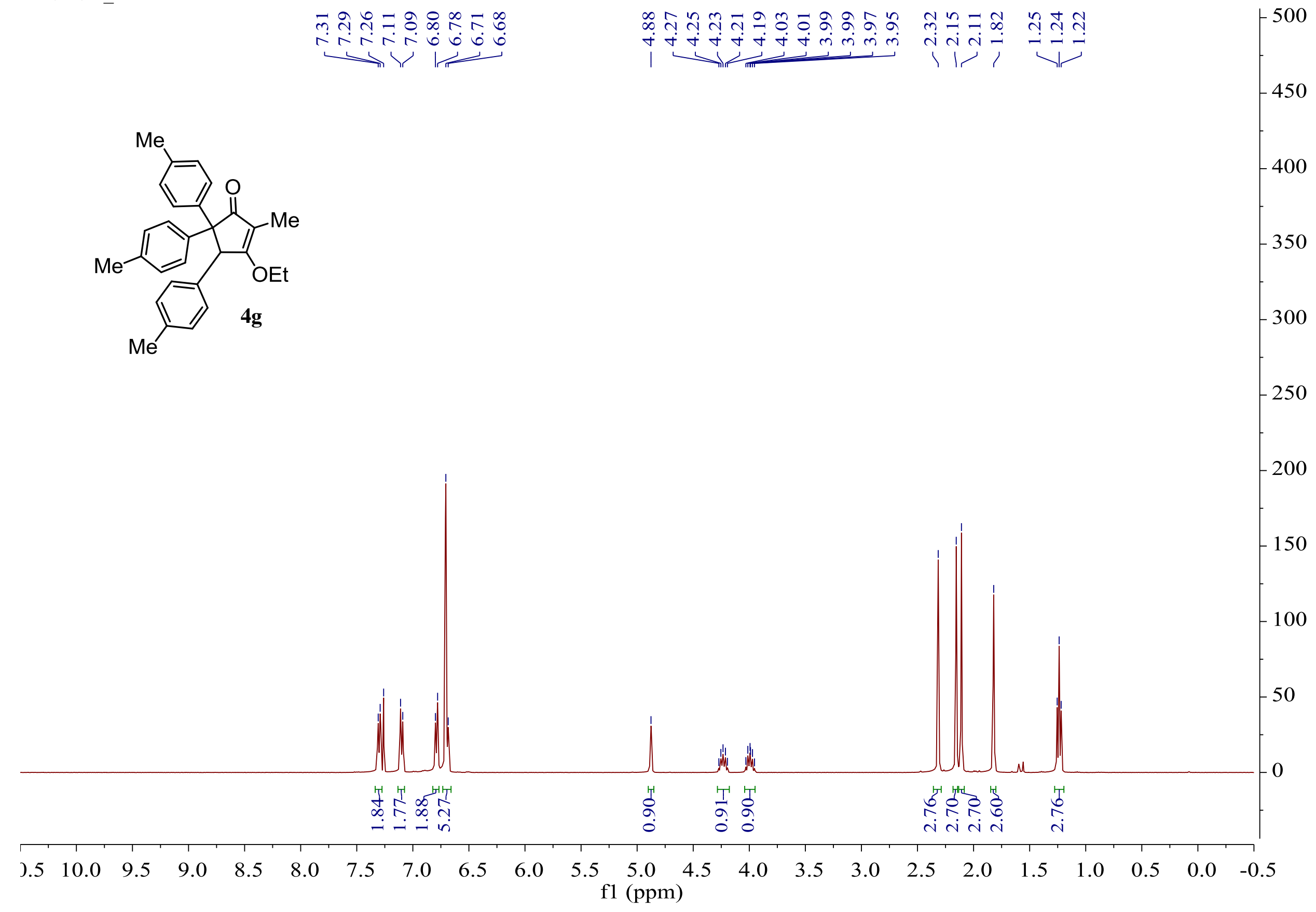



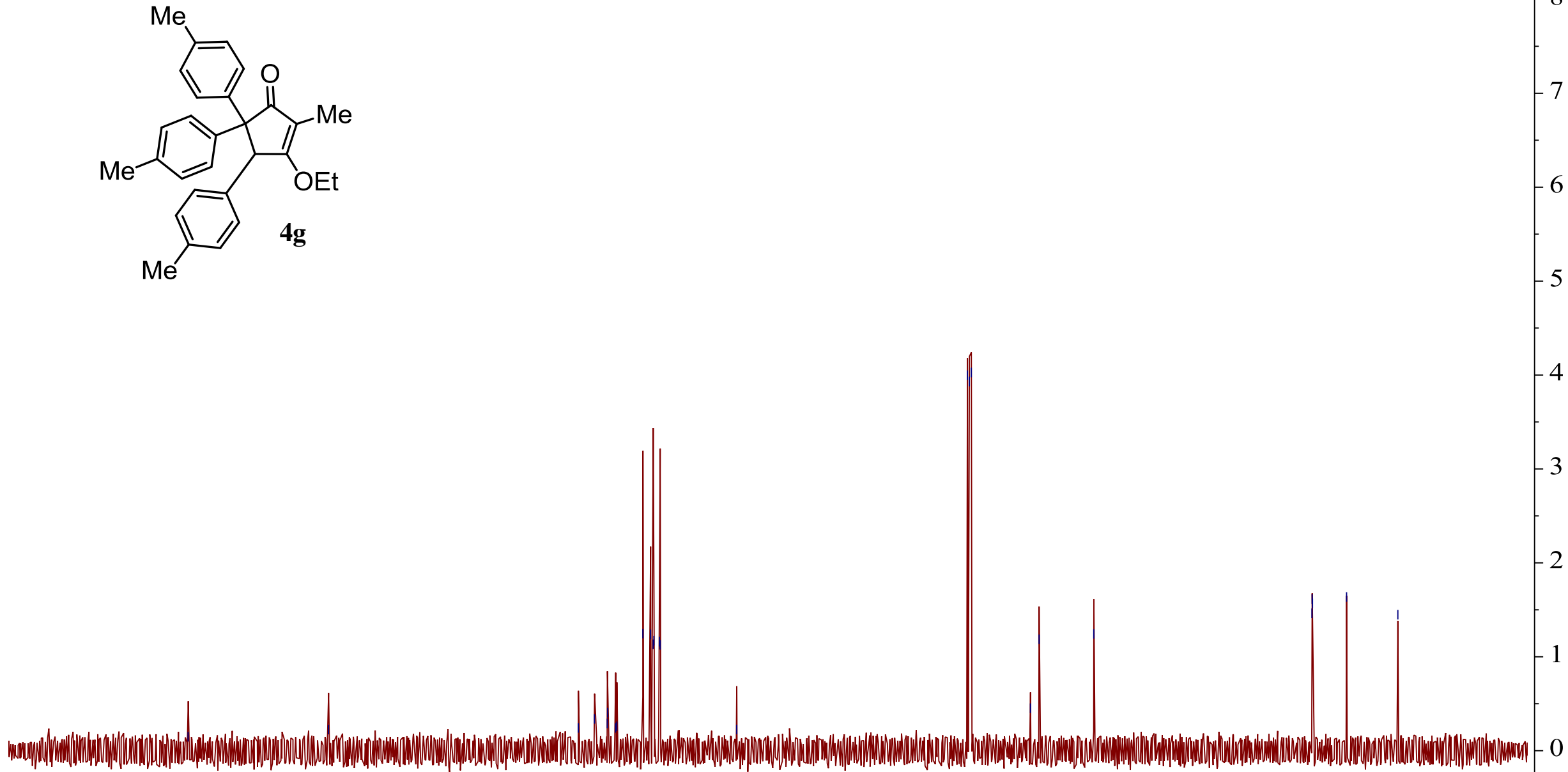


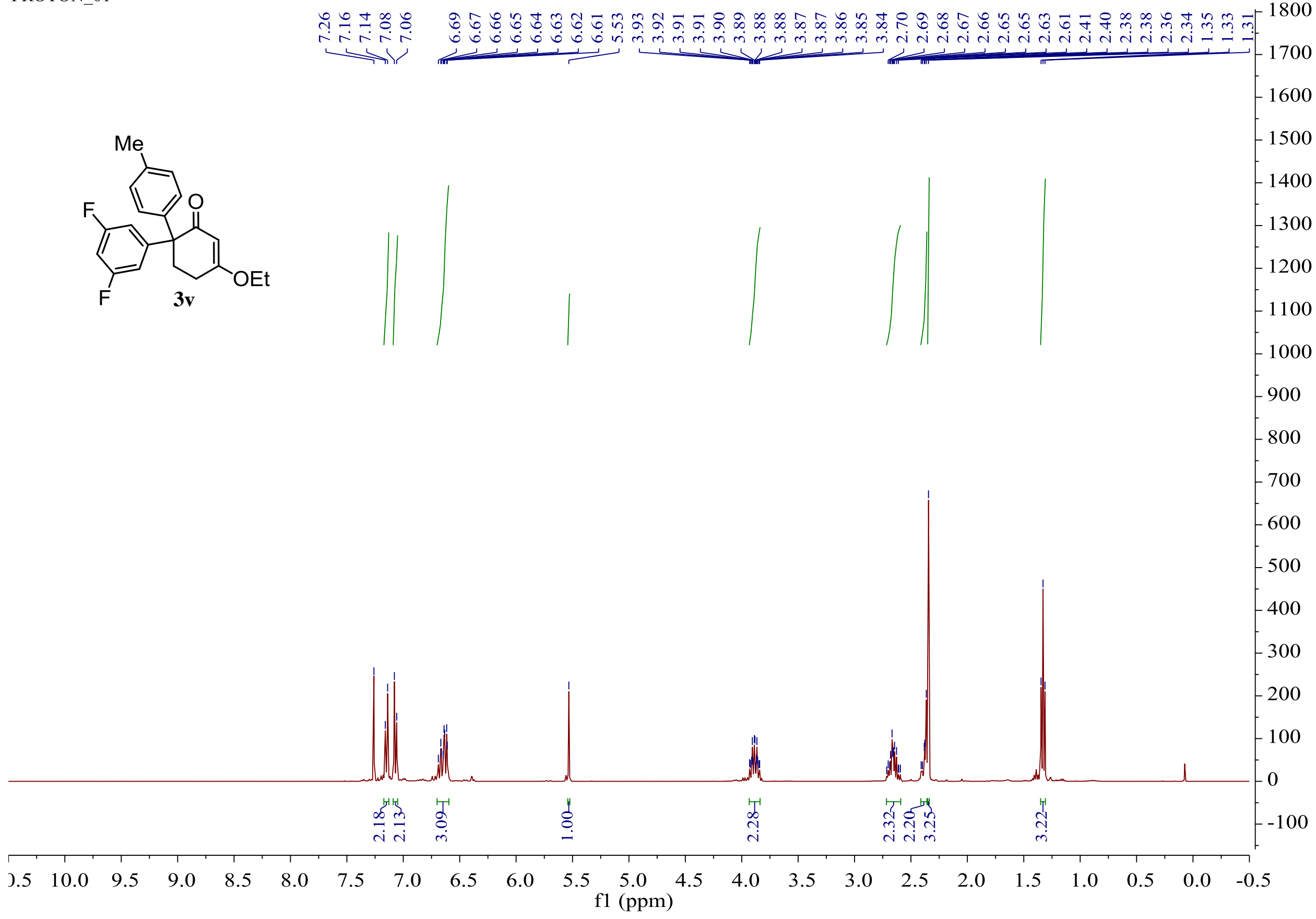


${ }^{13} \mathrm{C}$ NMR spectrum of compound $3 \mathrm{v}$

$\infty$
$\infty$
$\infty$
$\infty$
$\stackrel{1}{\mid}$

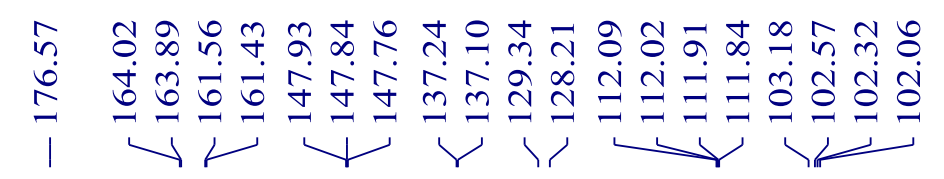

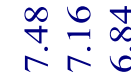

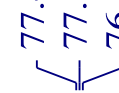

$\begin{array}{cc}0 & 0 \\ 0 & i \\ \dot{0} & \infty \\ 0 & n \\ 1 & 1\end{array}$

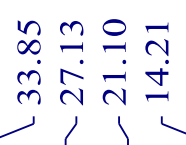

$-500$
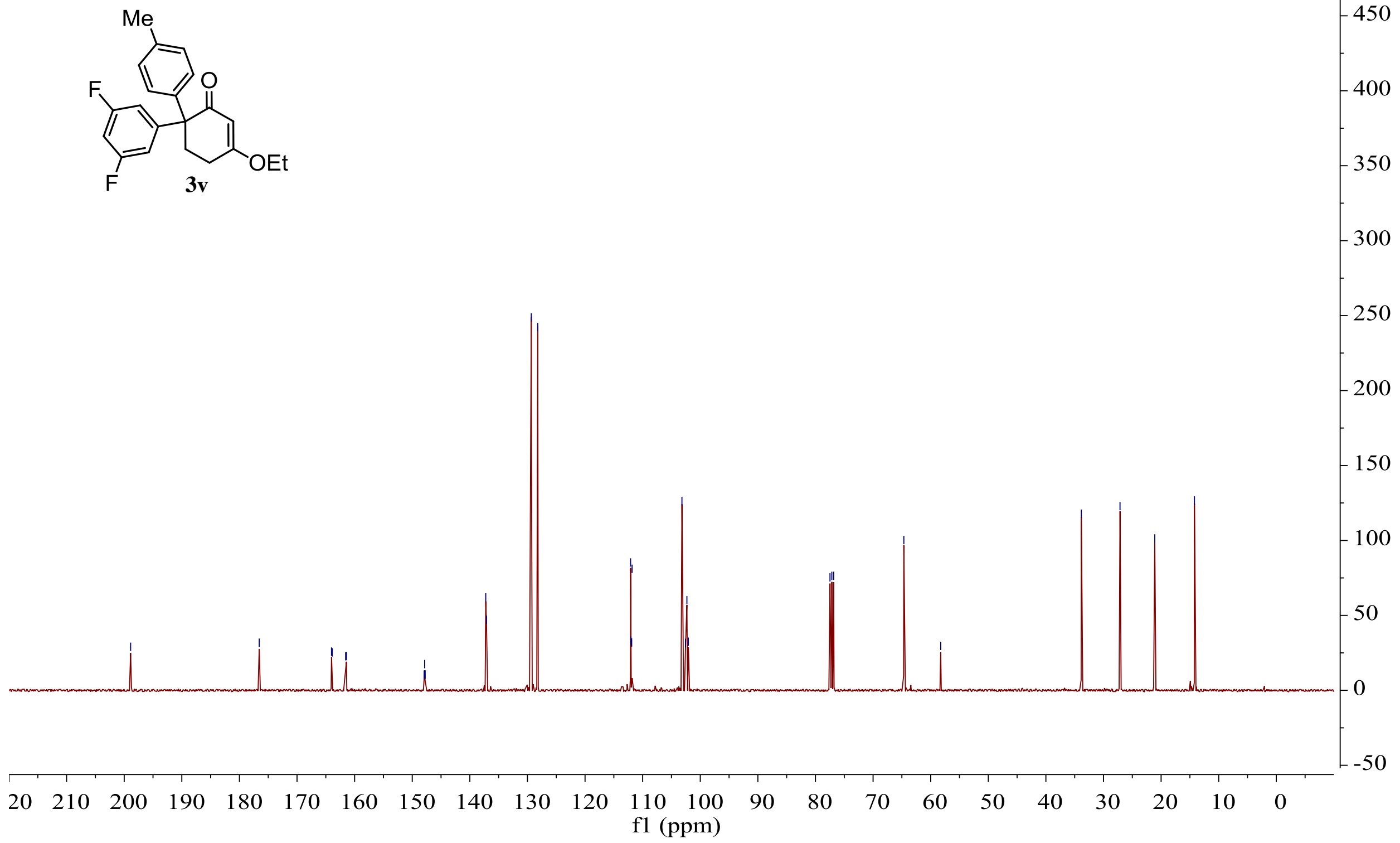
${ }^{19} \mathrm{~F}$ NMR spectrum of compound $3 \mathrm{v}$
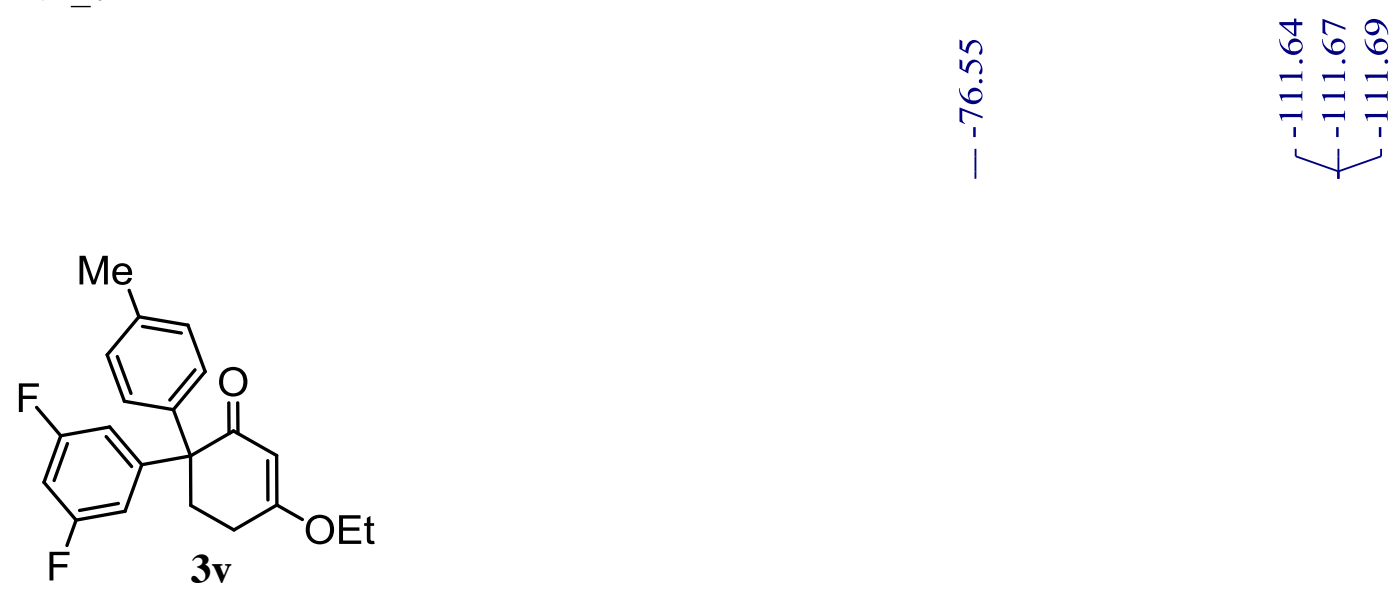

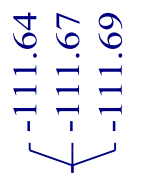

40000

$-38000$

$-36000$

$-34000$

$-32000$

30000

28000

26000

24000

22000

20000

18000

16000

14000

$-12000$

$-10000$

8000

6000

4000

2000

0

$\begin{array}{lllllllllllllllllllllllllll}0 & 20 & 10 & 0 & -10 & -20 & -30 & -40 & -50 & -60 & -70 & -80 & -90 & -100 & -110 & -120 & -130 & -140 & -150 & -160 & -170 & -180 & -190 & -200\end{array}$ f1 (ppm) 
${ }^{1} \mathrm{H}$ NMR spectrum of compound $3 \mathrm{w}$

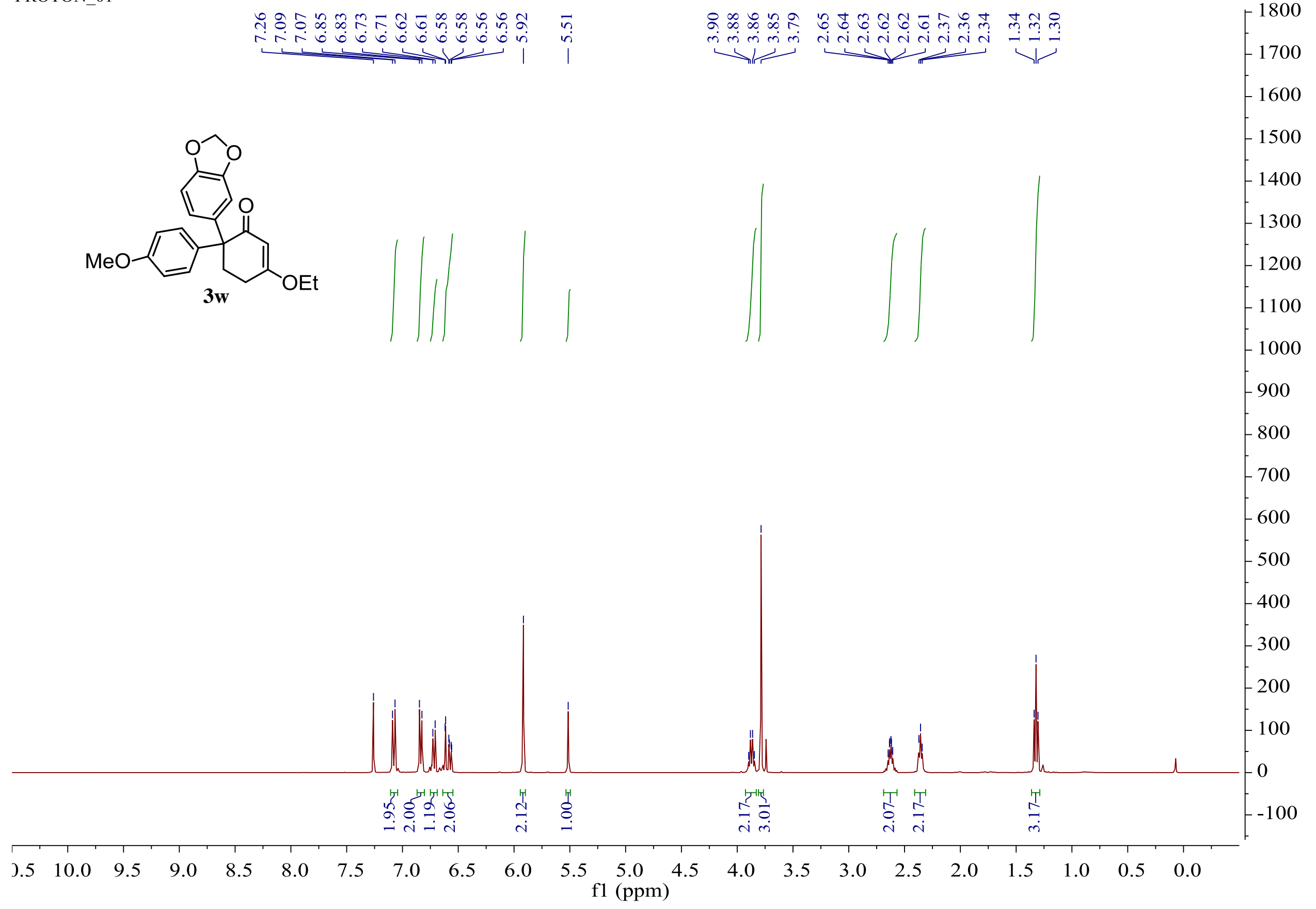


CARBON_01

${ }^{13} \mathrm{C}$ NMR spectrum of compound $3 w$

กิ

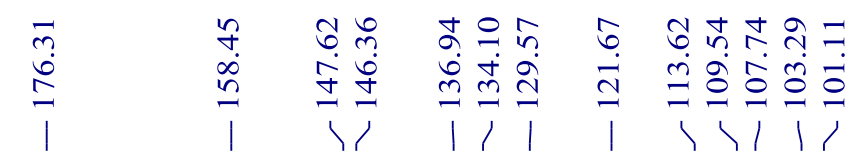

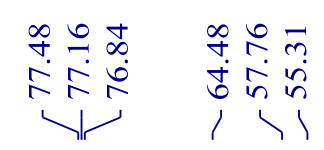

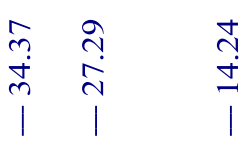

320

$-300$
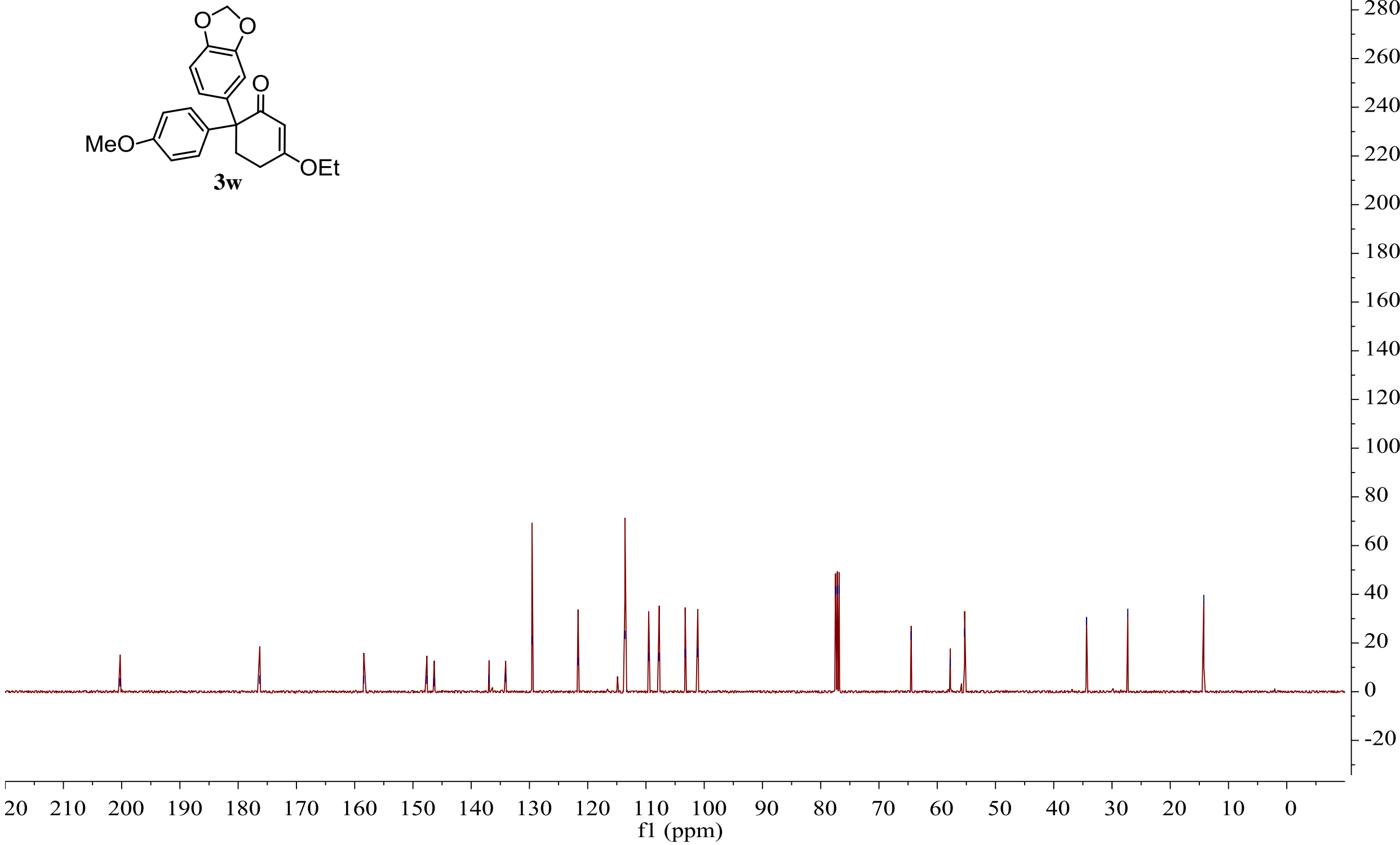
${ }^{1} H$ NMR spectrum of compound $3 x$

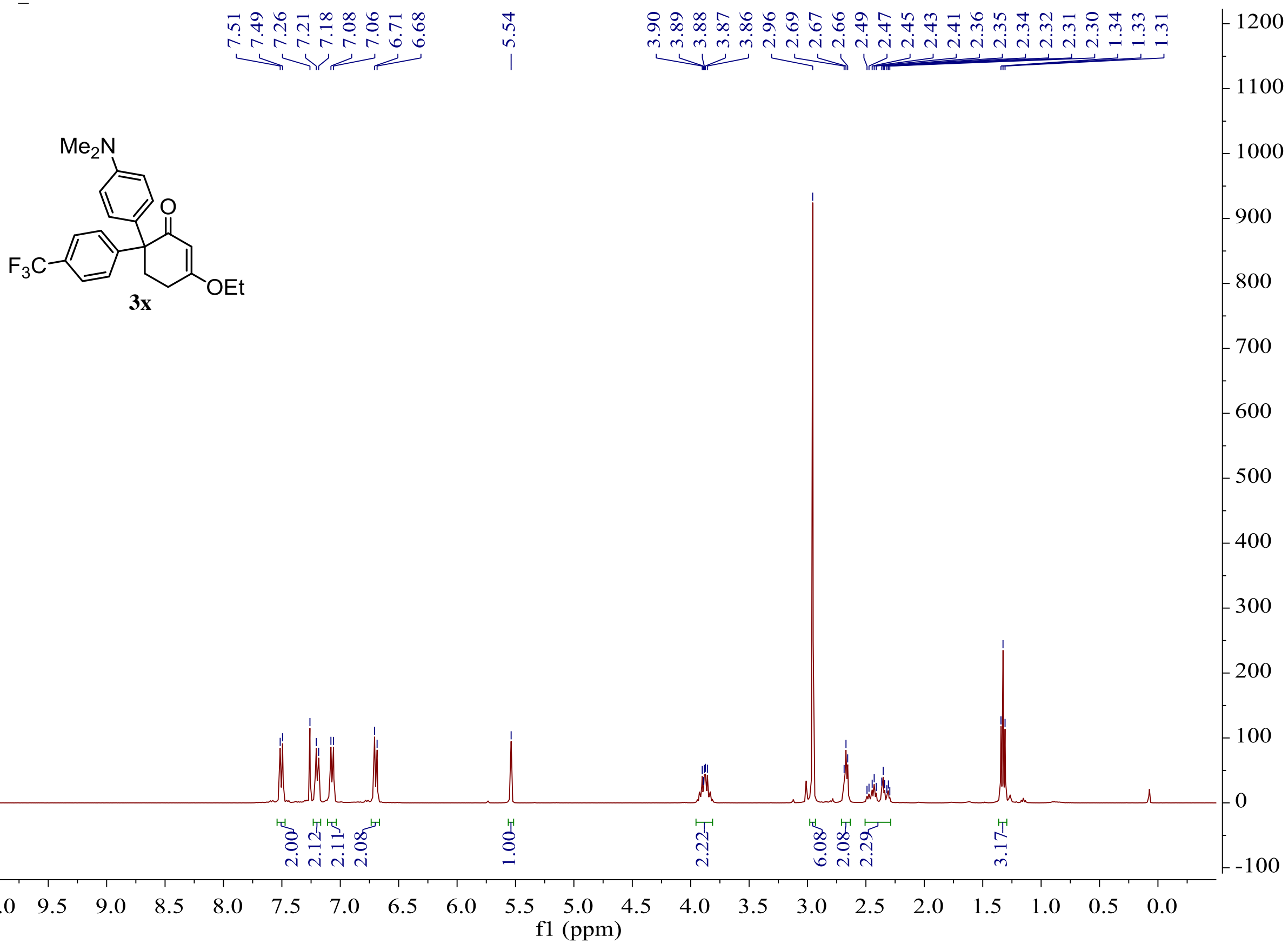


CARBON_01

${ }^{13} \mathrm{C}$ NMR spectrum of compound $3 \mathrm{x}$

㐫

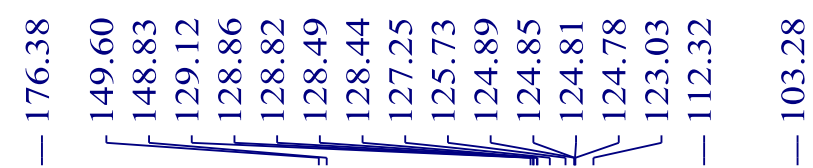

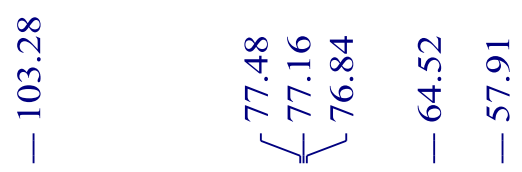

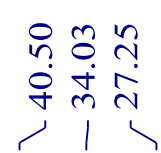

$\stackrel{\substack{n \\ \mathfrak{z}}}{i}$

-180
-170

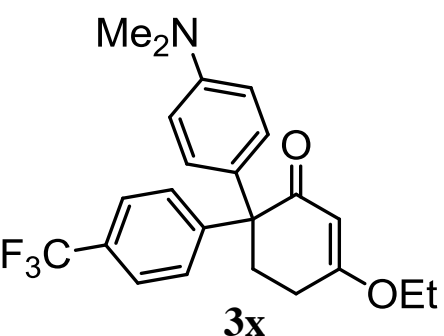

3x

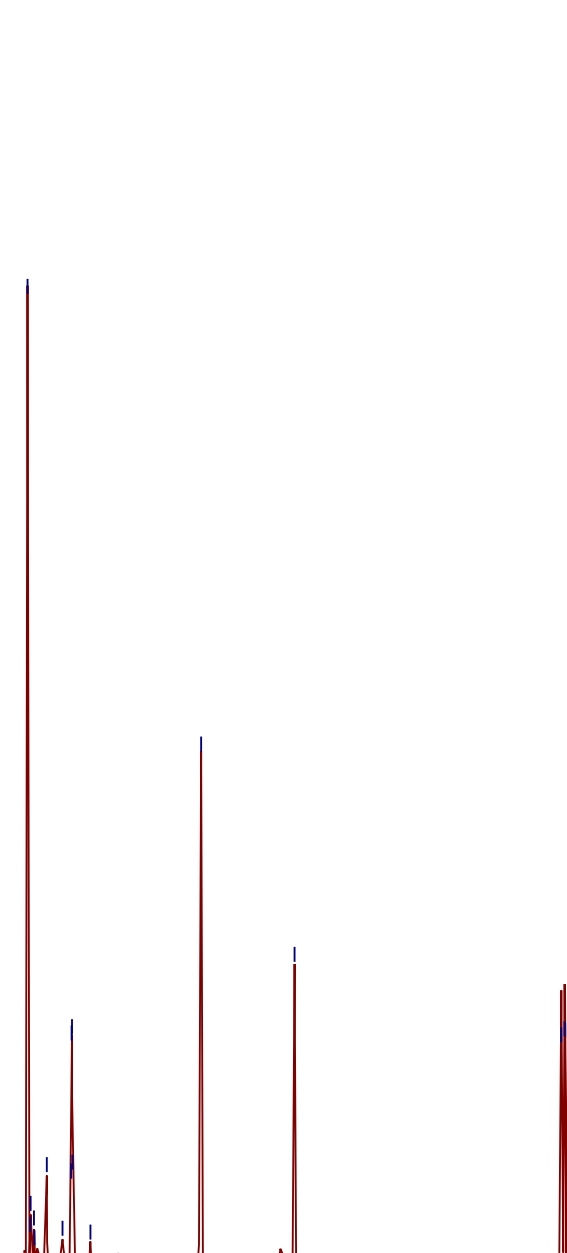

160

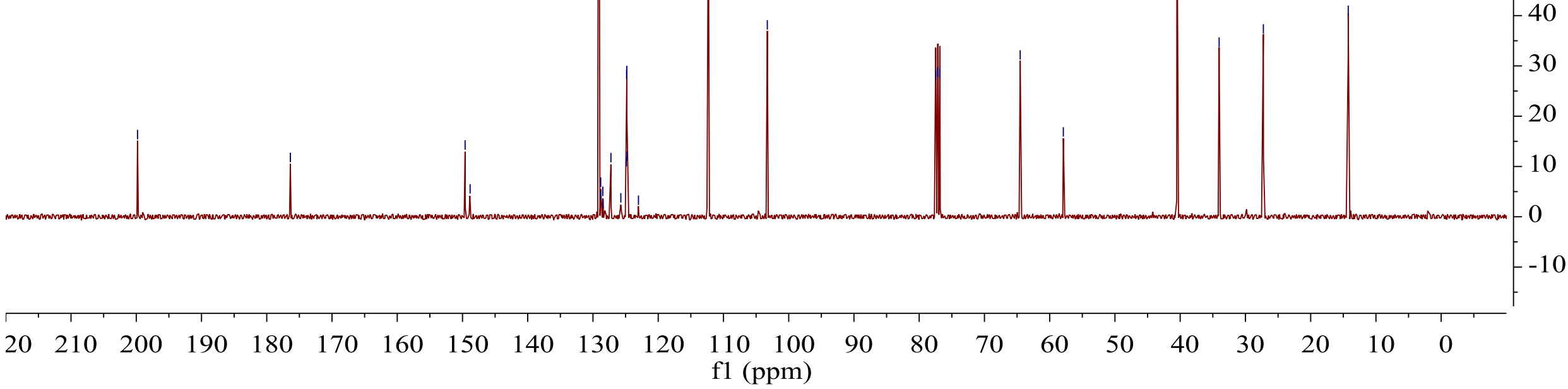


FLUORINE_01

\section{${ }^{19}$ F NMR spectrum of compound $3 x$}
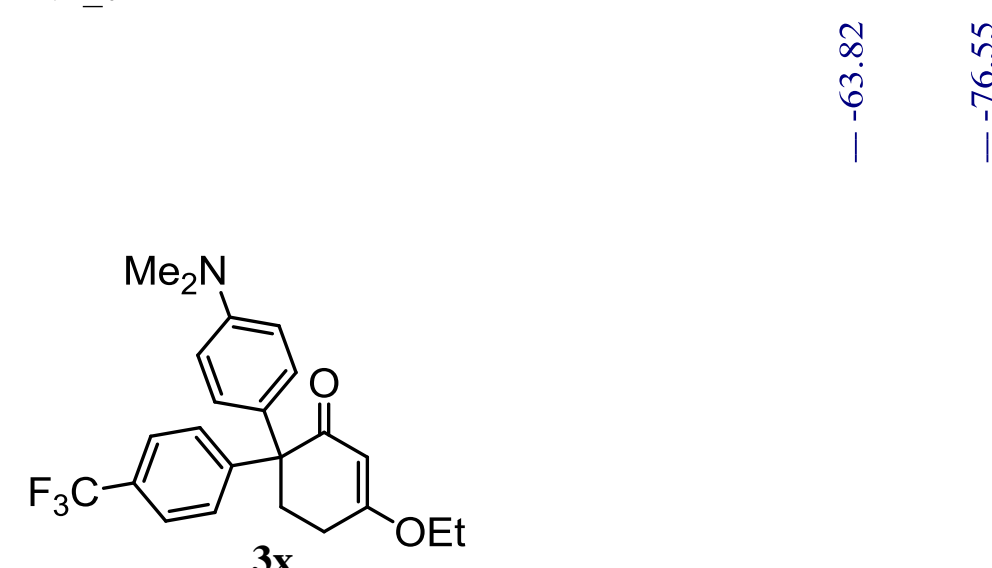

32000

30000

28000

26000

24000

22000 


\section{${ }^{1} \mathrm{H}$ NMR spectrum of compound $3 \mathrm{y}$}

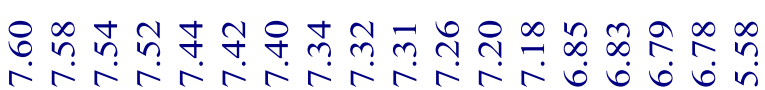

(

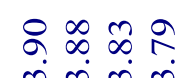

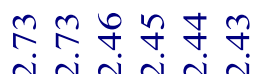

$\sqrt{4} 444 \pi$

חृ?
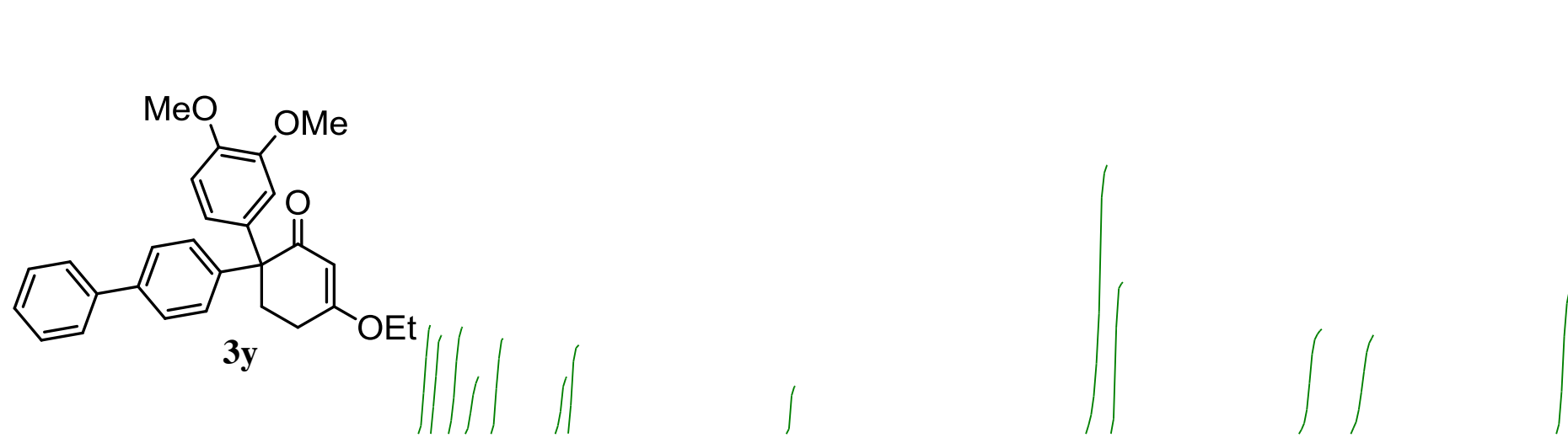

700

600

500

400

300

200

100

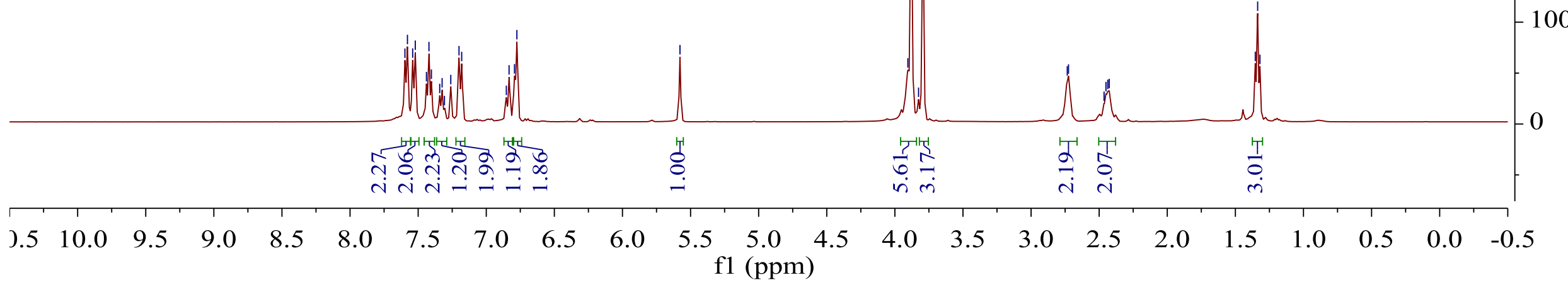




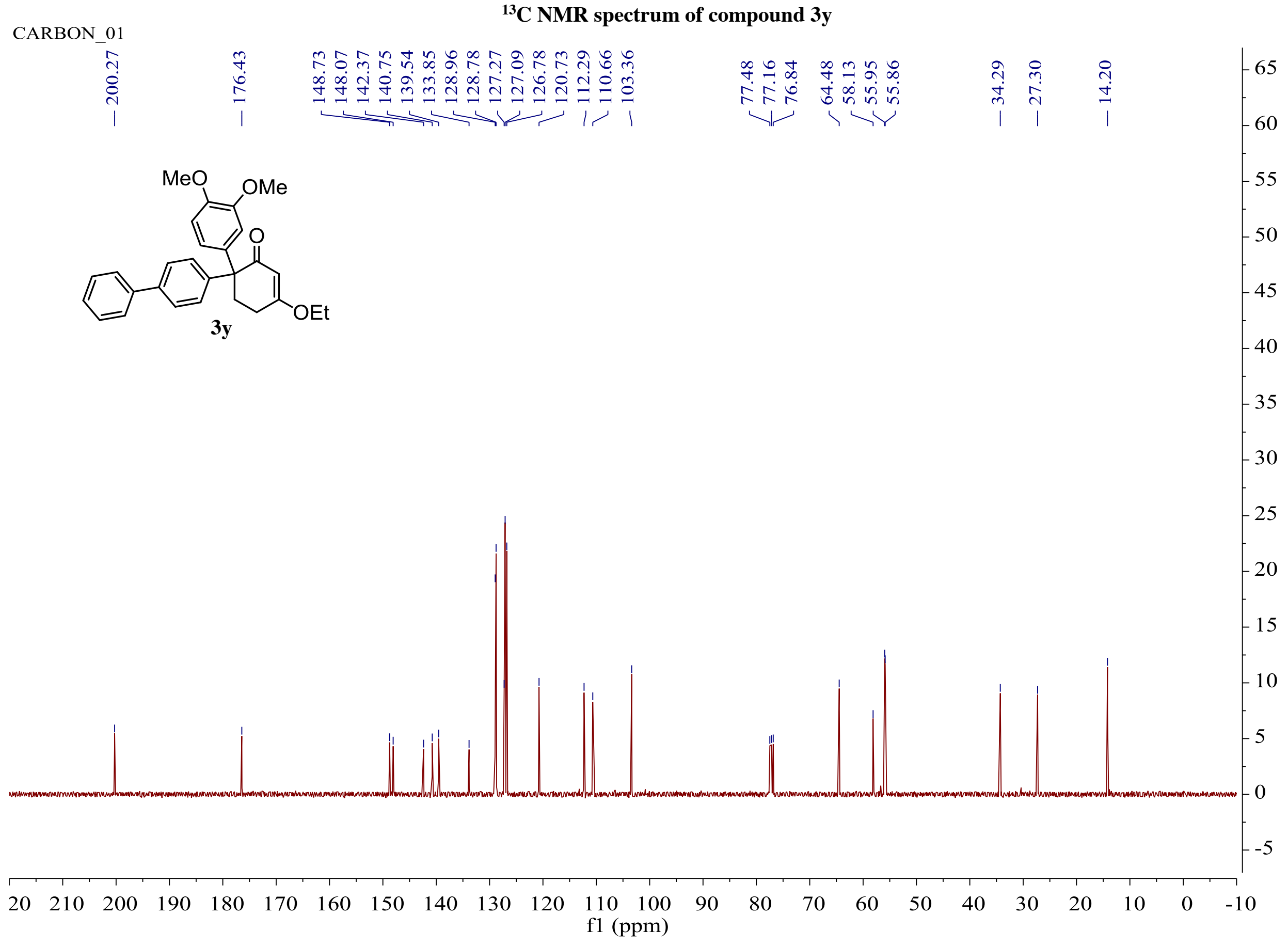




\section{${ }^{1}$ H NMR spectrum of compound $3 z$}

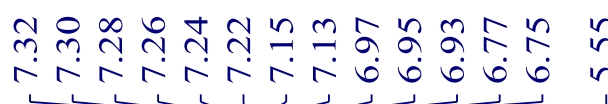

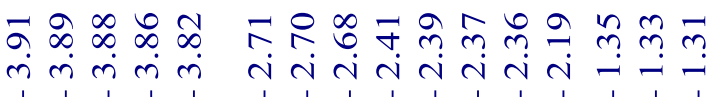

$\longrightarrow$ L

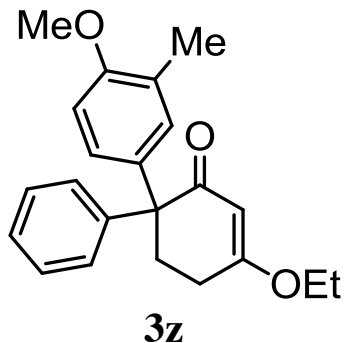

$3 z$
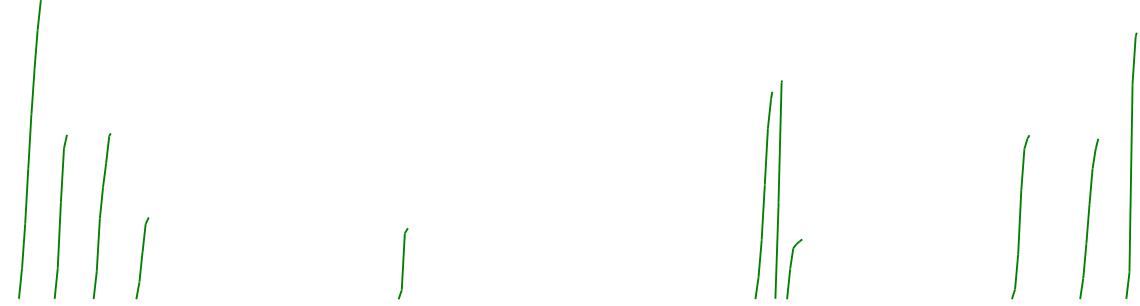

600

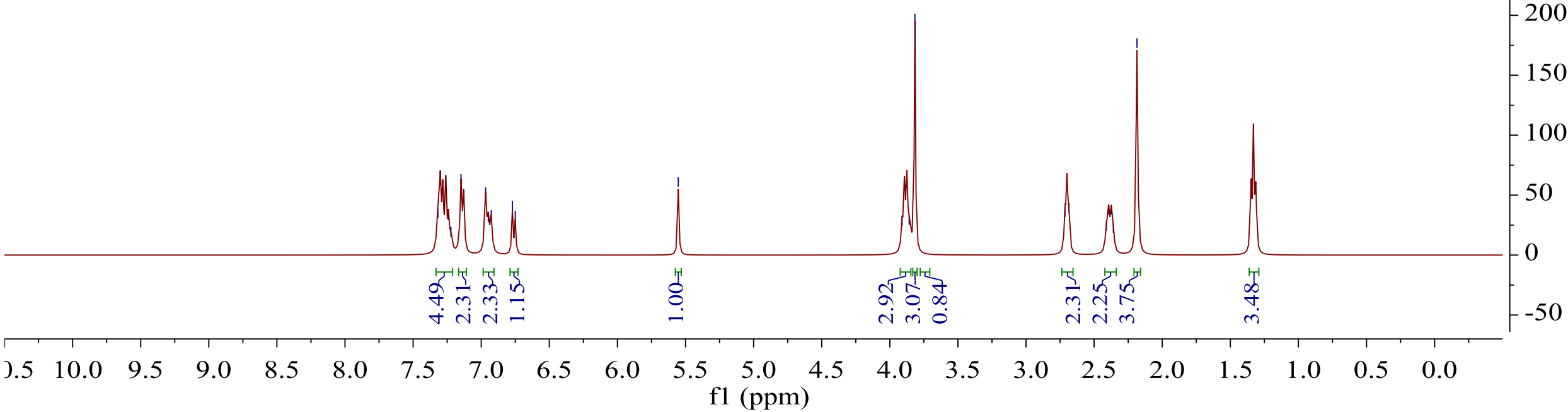




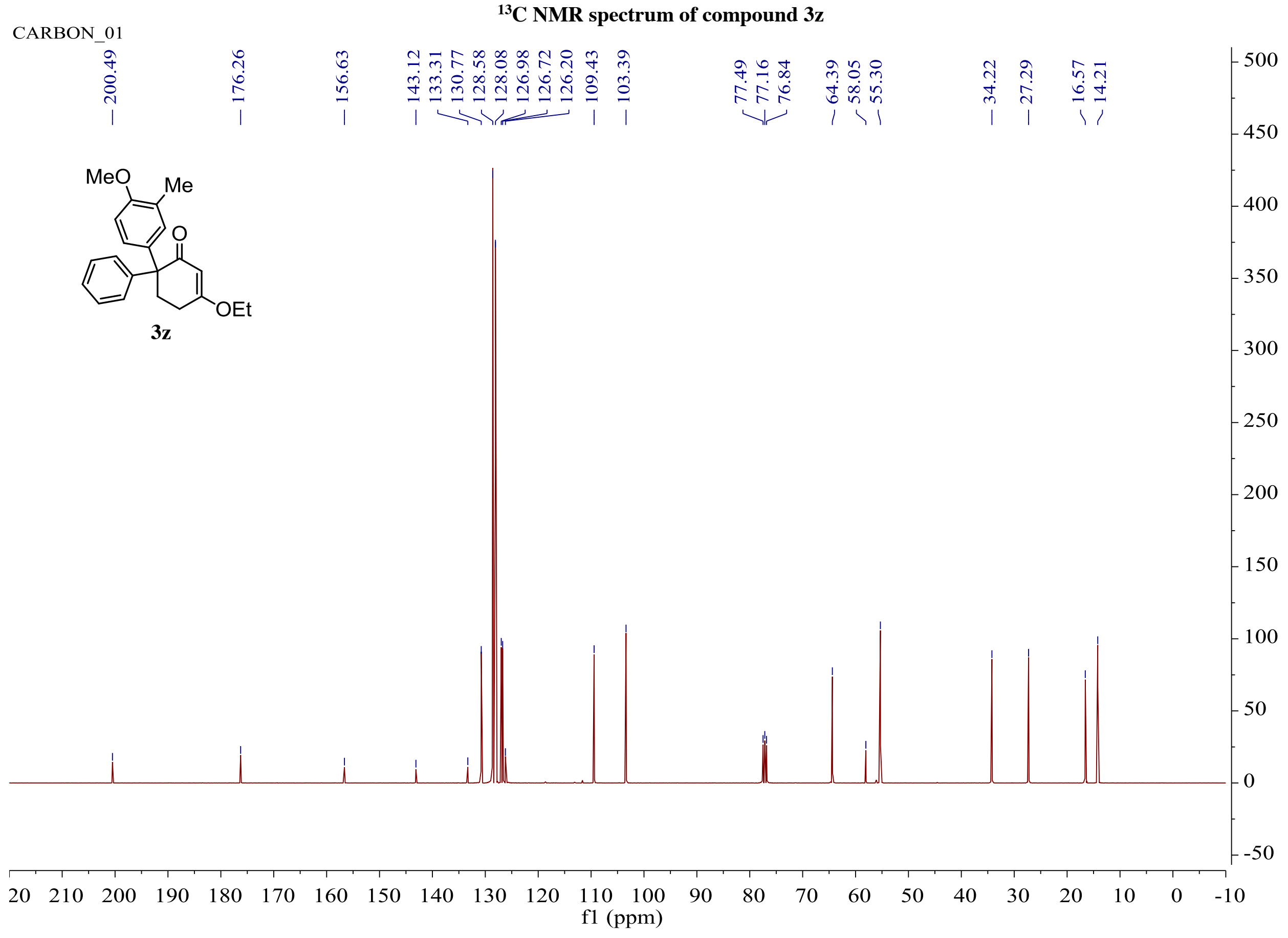


${ }^{1} \mathrm{H}$ NMR spectrum of compound $4 \mathrm{~h}$

PROTON 01

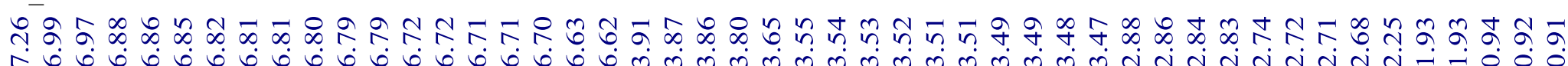

(1)

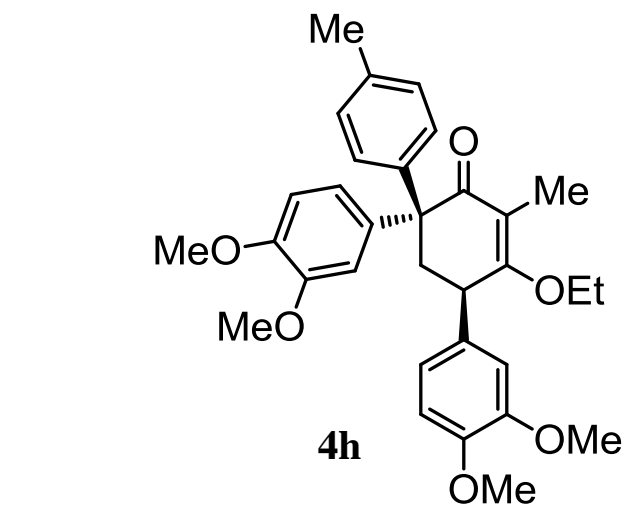

$0.5 \quad 10.0 \quad 9.5$

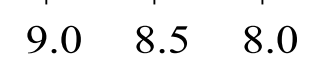

7.5

$\begin{array}{ll}7.0 & 6.5\end{array}$

$6.0 \quad 5.5$

f1 (ppm) 
CARBON_01

${ }^{13} \mathrm{C}$ NMR spectrum of compound $4 \mathrm{~h}$

ลे

我

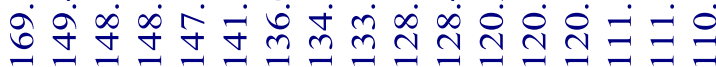

$\underbrace{\sim}_{m}$

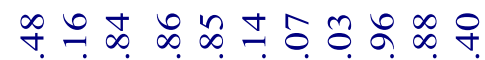

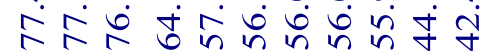

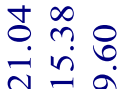

$-55$

ए।

11
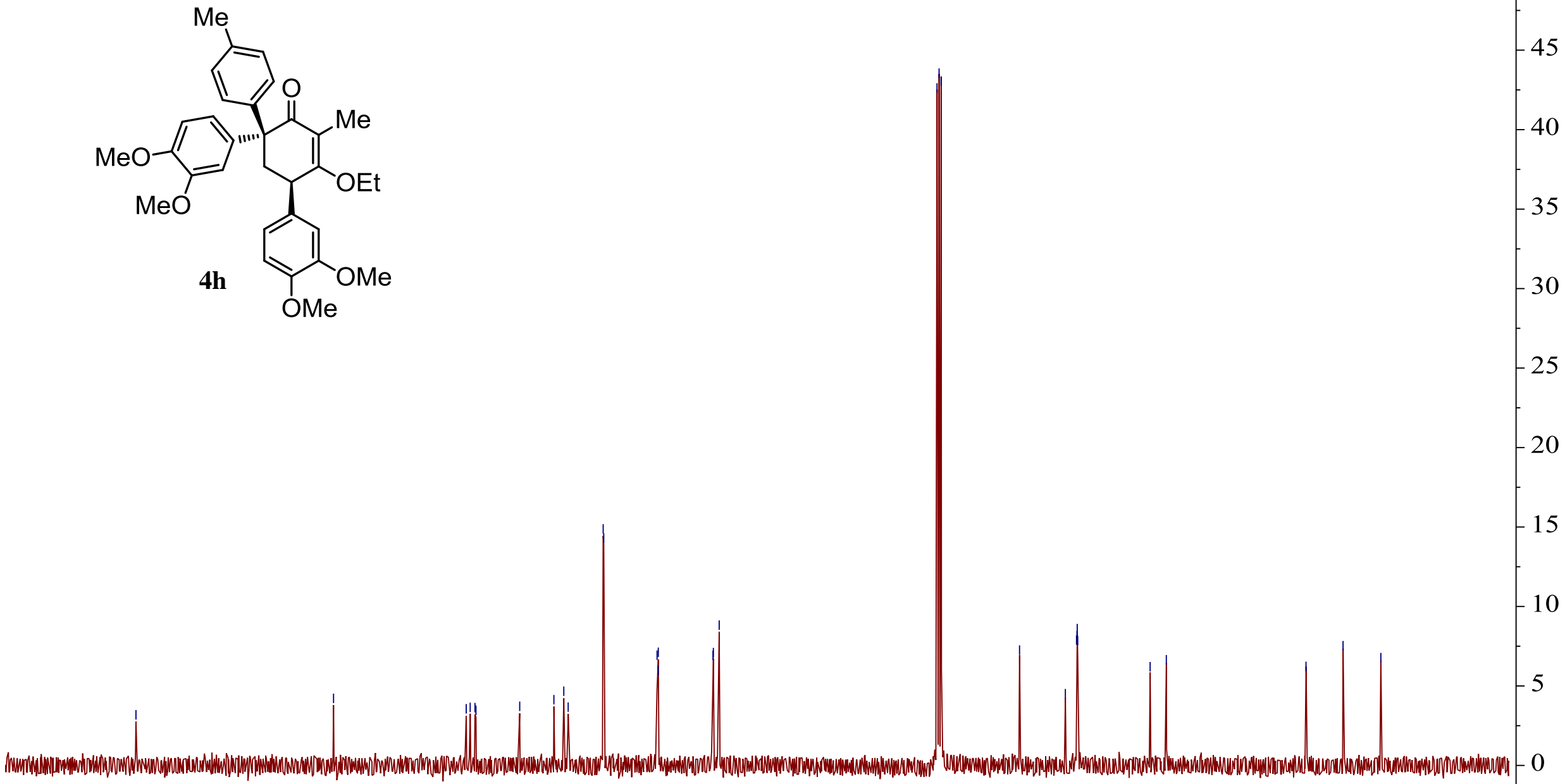

$\begin{array}{lllllllllllll}20 & 210 & 200 & 190 & 180 & 170 & 160 & 150 & 140 & 130 & 120 & 110 & 100\end{array}$ f1 (ppm) 


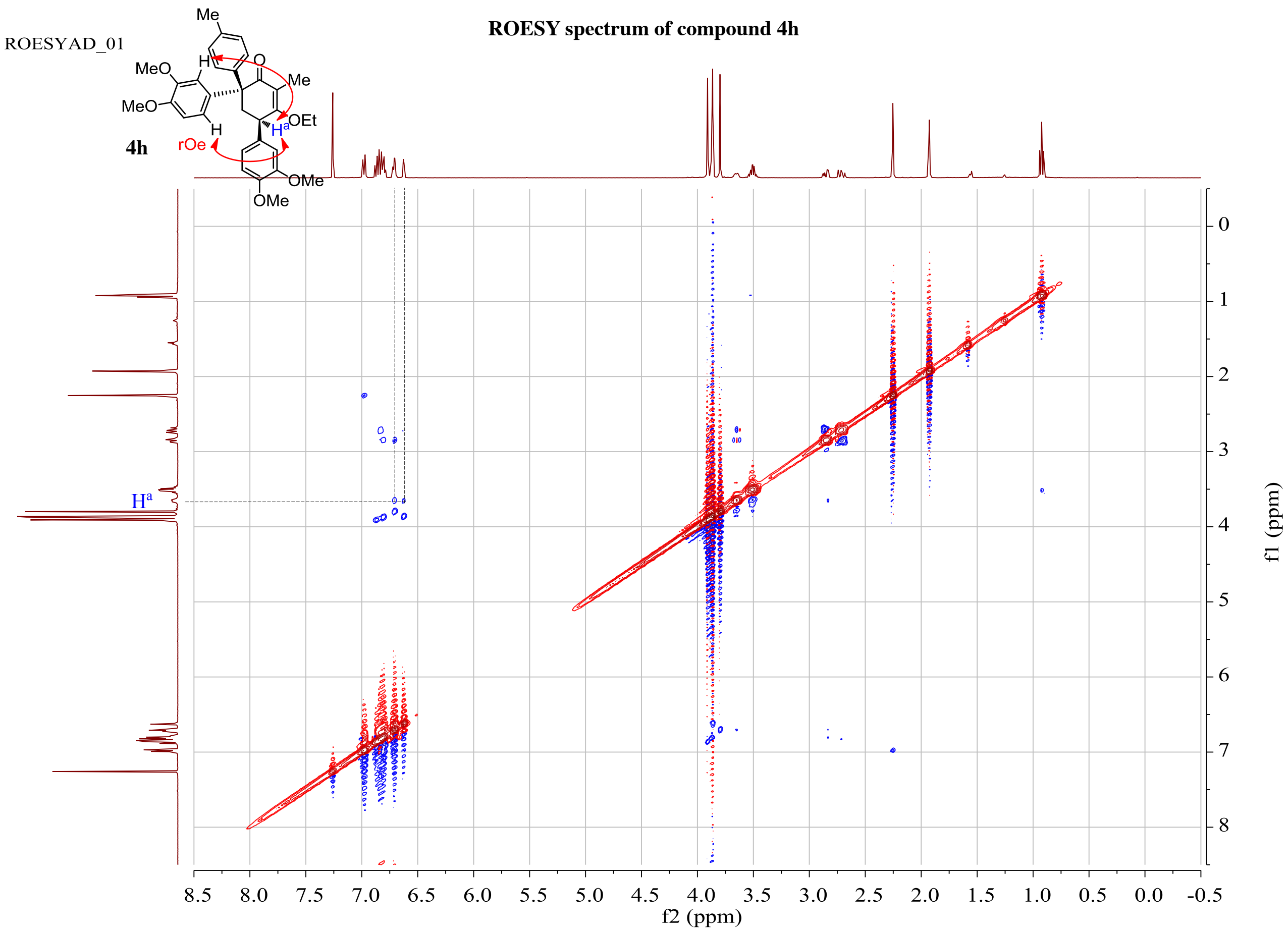

S-118 


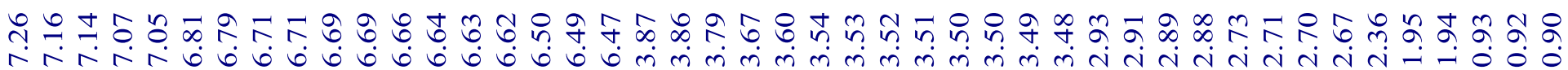

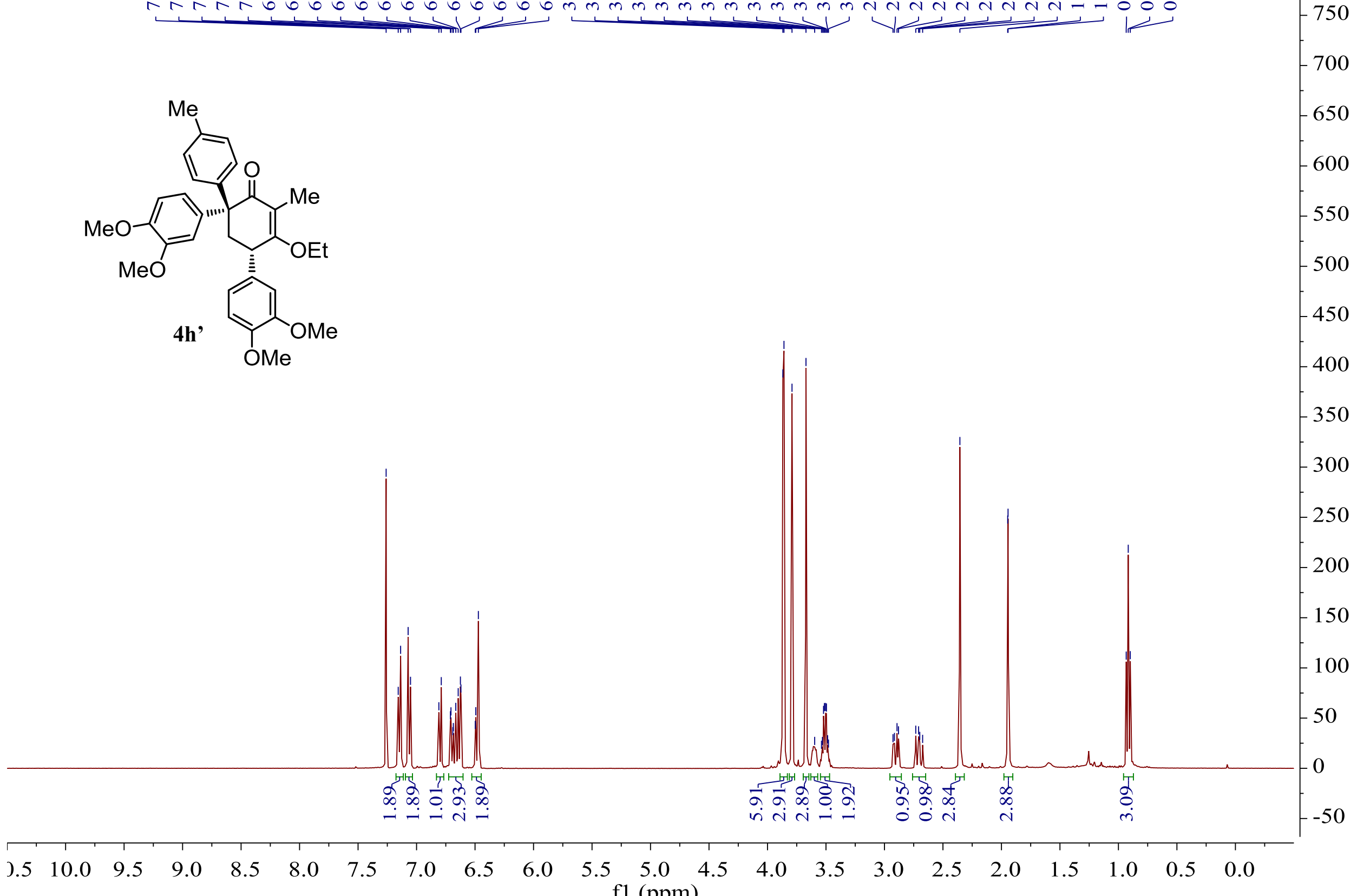


CARBON_01

${ }^{13} \mathrm{C}$ NMR spectrum of compound $4 \mathrm{~h}$,

๙ू.

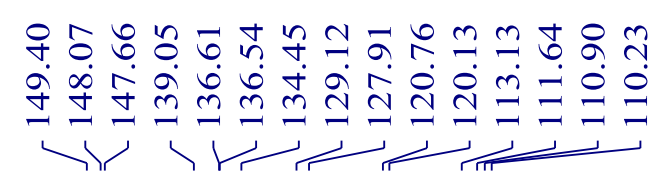

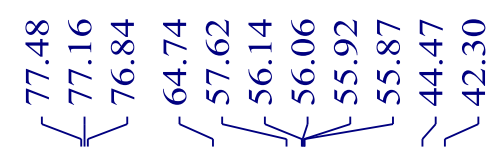

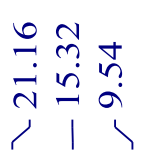

$-85$

$\stackrel{7}{a}$<smiles>CCOC1=C(C)C(=O)C(c2ccc(OC)cc2)(c2ccc(OC)c(OC)c2)C[C@H]1c1ccc(OC)c(OC)c1</smiles> 


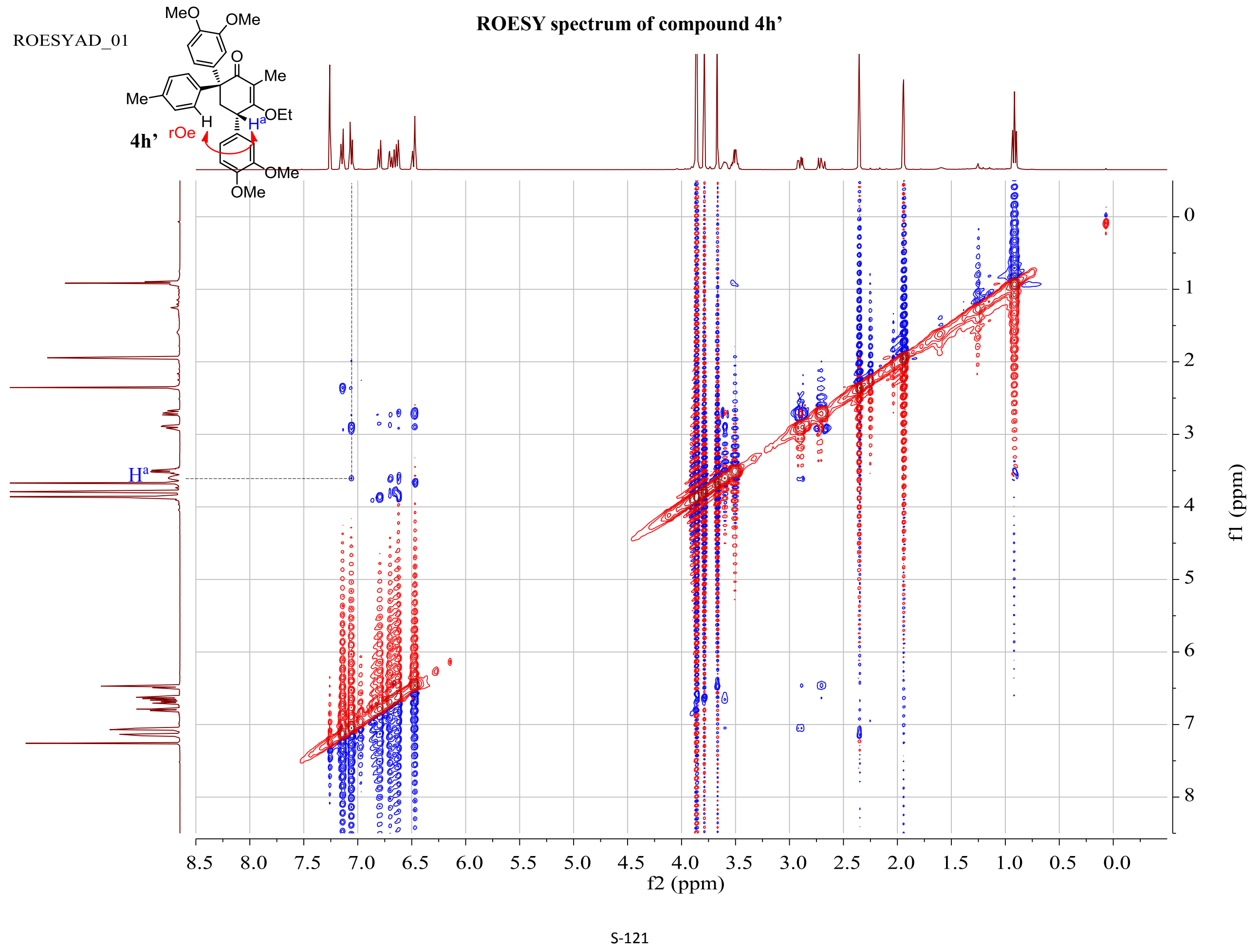


PROTON_01

\section{${ }^{1} \mathrm{H}$ NMR spectrum of compound 6}

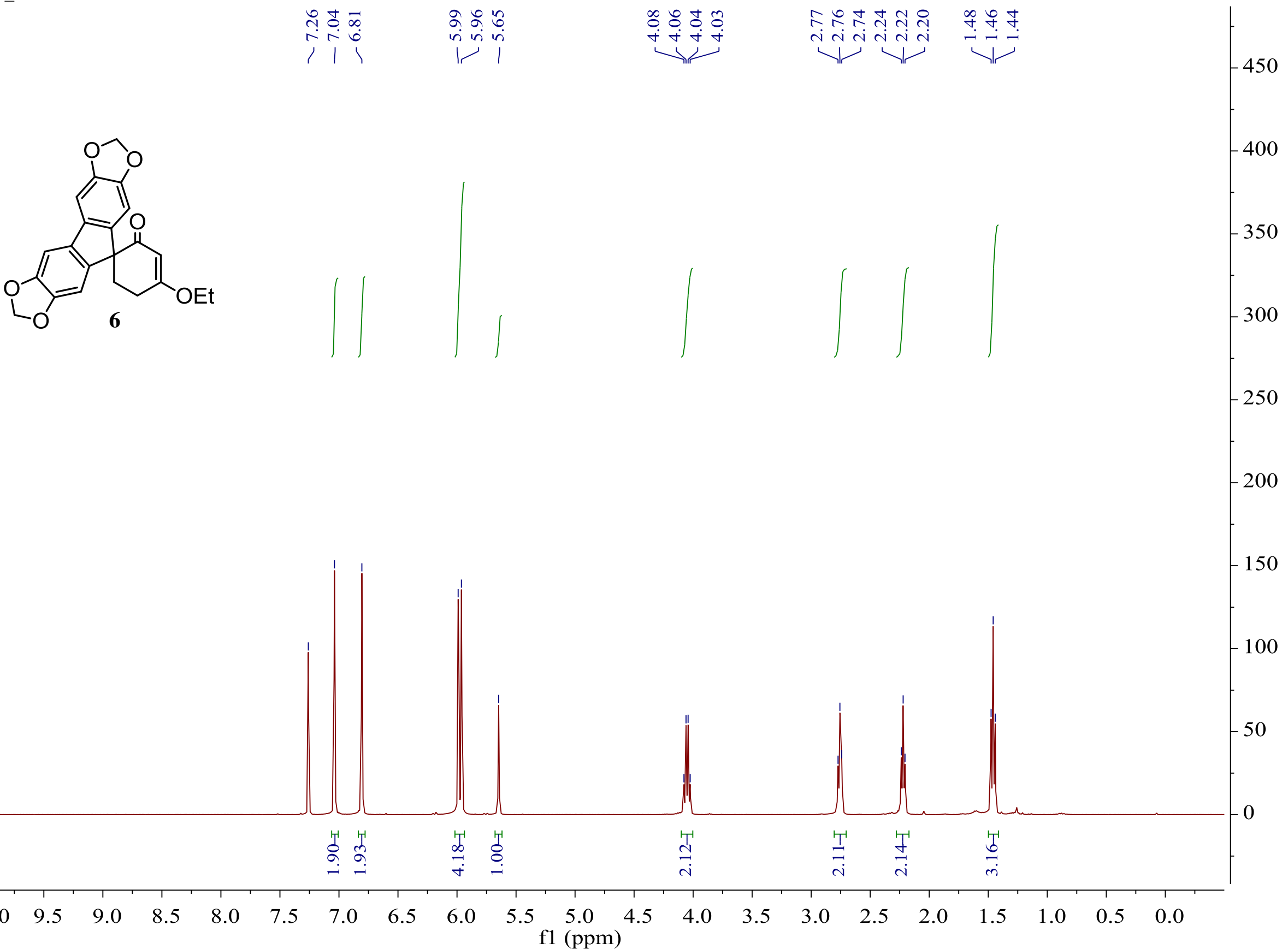


CARBON_01

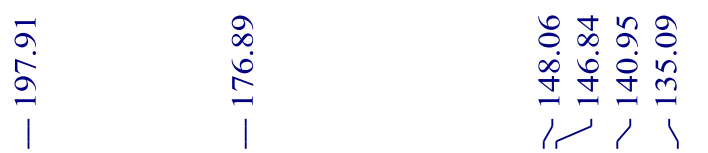

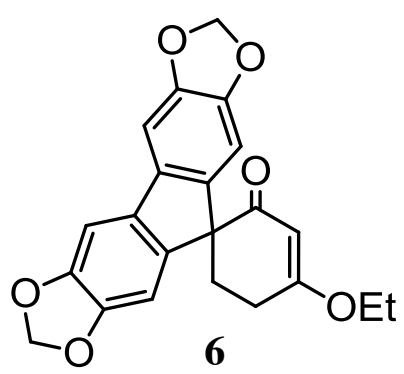

\section{${ }^{13} \mathrm{C}$ NMR spectrum of compound 6}

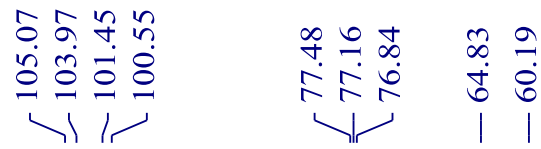

8ิ ก

i

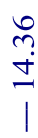

50

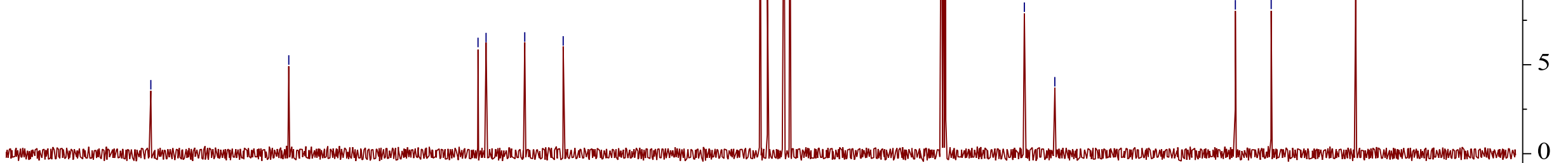

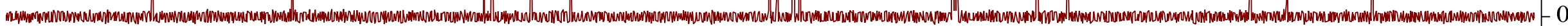

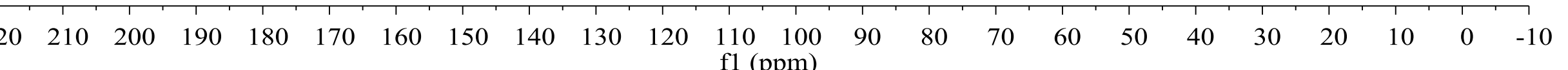


${ }^{1} \mathrm{H}$ NMR spectrum of compound 7

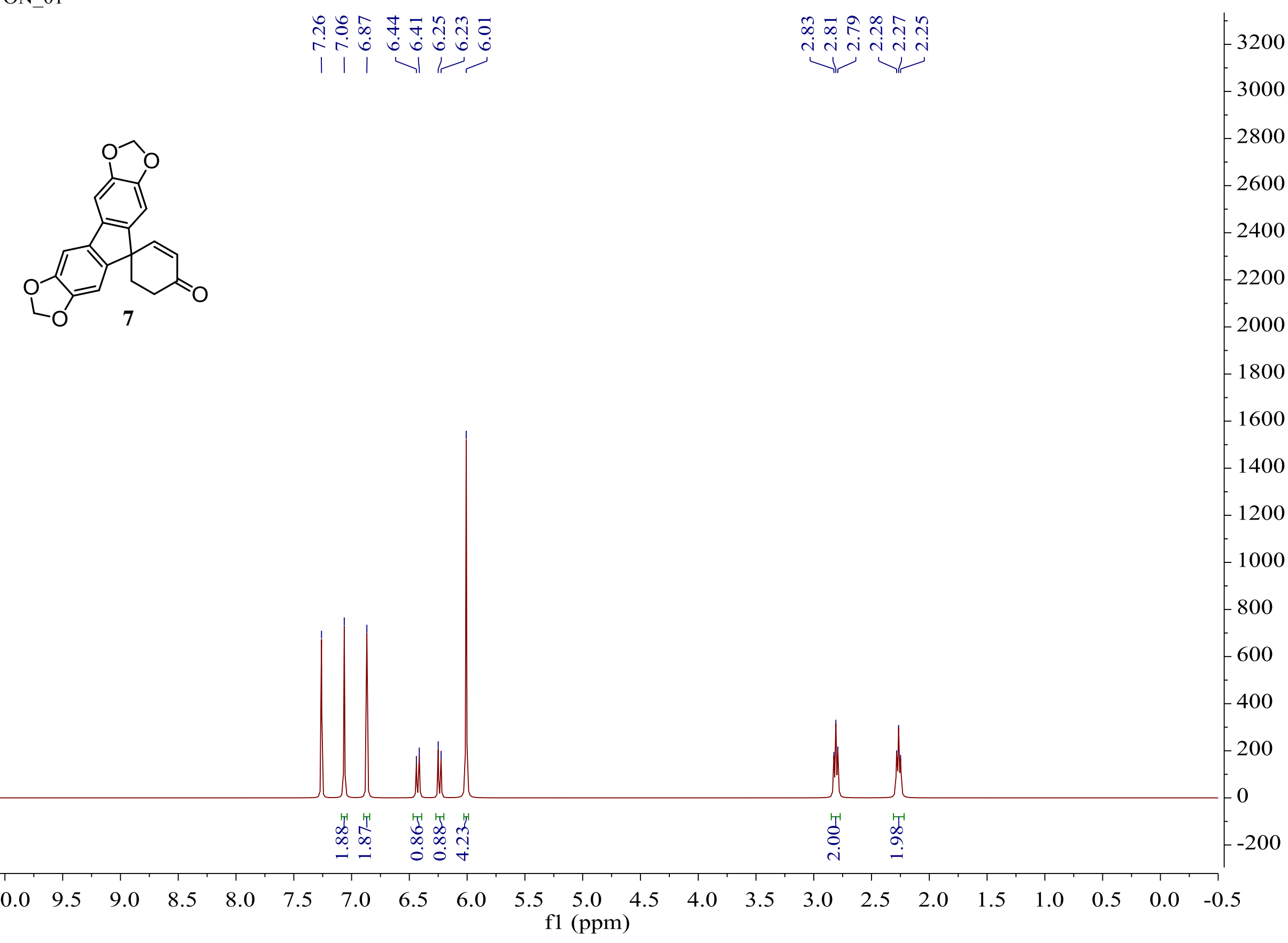


CARBON 01

2
$\infty$
$a$
a

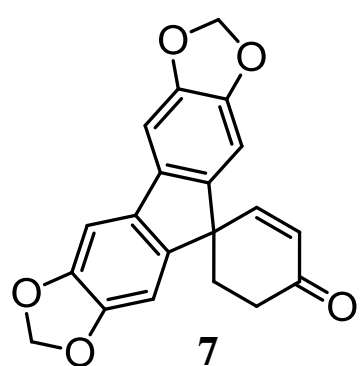

\section{${ }^{13}$ C NMR spectrum of compound 7}

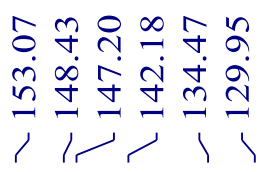

$\begin{array}{ccc}0 & n \\ n & 0 & m \\ n & - & 0 \\ 0 & 0 & 0\end{array}$

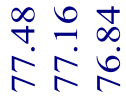

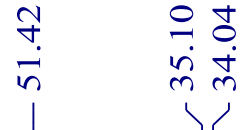

$-55$

$-50$ 
PROTON 01

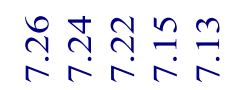

${ }^{1} \mathrm{H}$ spectrum of compound 8

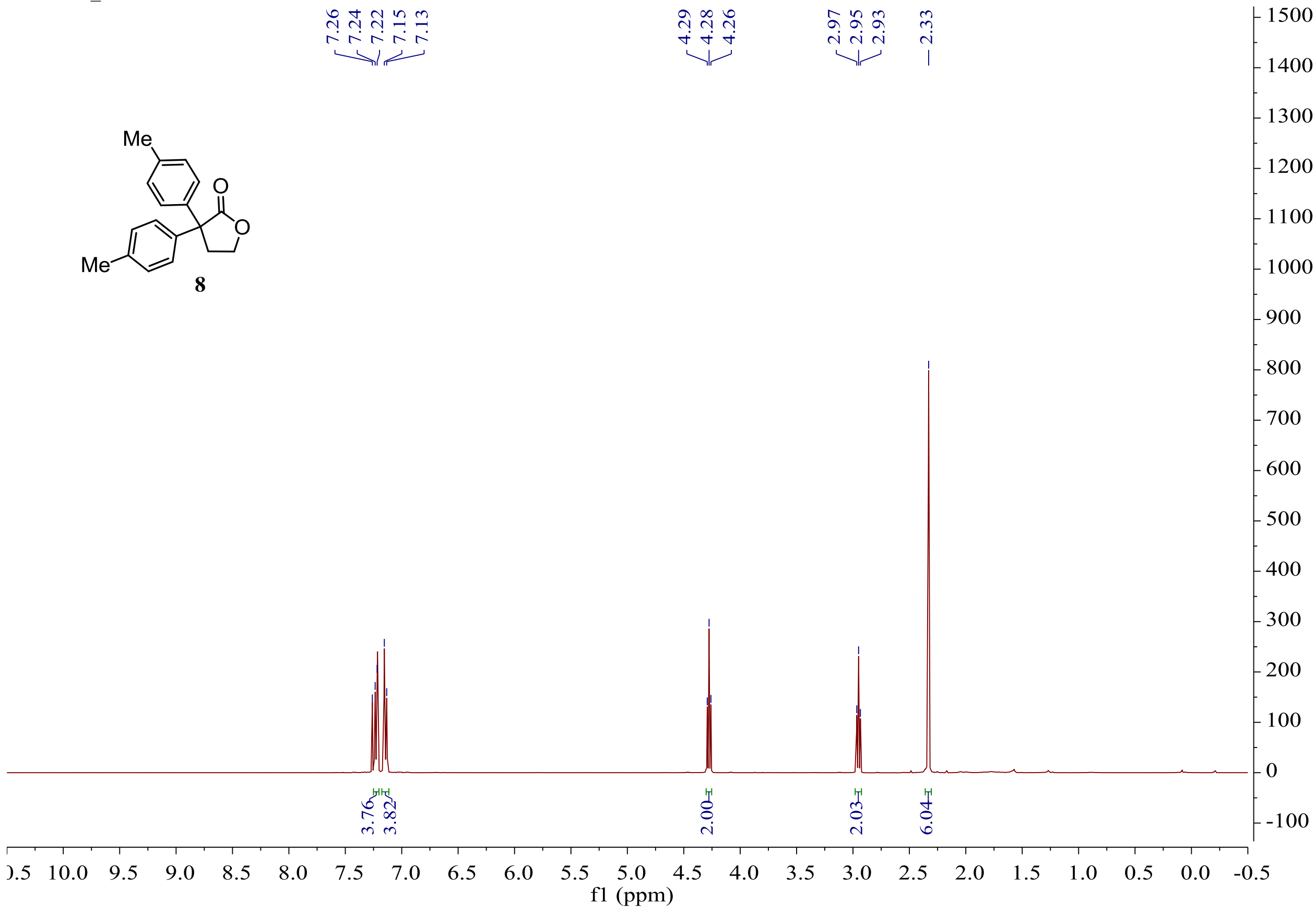


CARBON 01

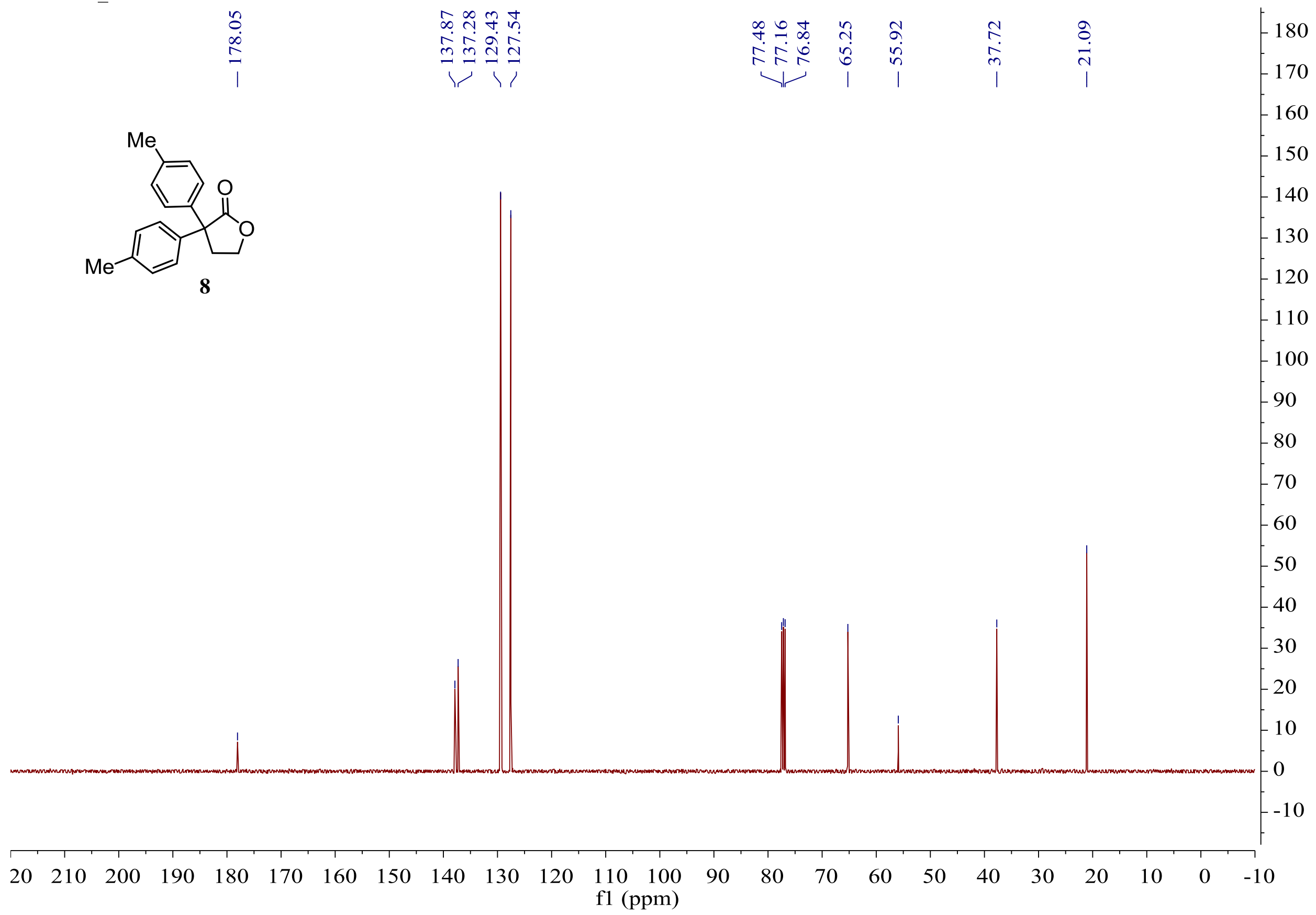

\section{${ }^{13} \mathrm{C}$ spectrum of compound 8}

